\title{
Functionalisation of carbon nanotubes for molecular electronics
}

Natalie O. V. Plank

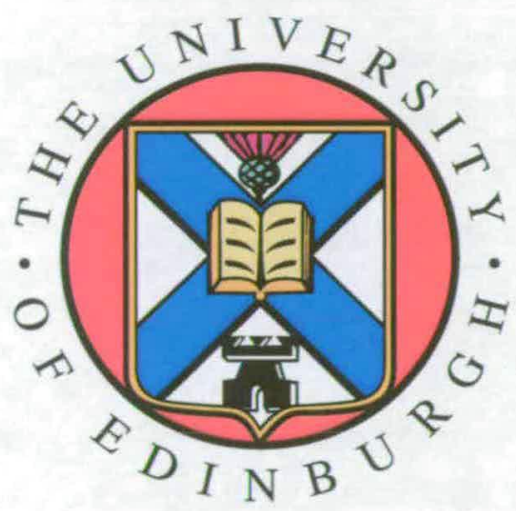

A thesis submitted for the degree of Doctor of Philosophy.

The University of Edinburgh.

October 2005 


\section{Abstract}

Carbon nanotubes (CNTs) have been chemically functionalised for electronic device applications using plasma exposure processes. Two areas of CNT device have been investigated. Firstly the conversion of the inherent p-type field effect behaviour of the CNTs to n-type field effect behaviour and secondly to control the positioning of CNTs on a substrate.

To convert CNTs from p-type to n-type semiconductors, the chemically unreactive CNTs have been functionalised by exposure to fluorinated plasmas, both $\mathrm{CF}_{4}$ and $\mathrm{SF}_{6}$. Further functionalisation with 1,2-diaminoethane was then employed on CNTs fluorinated by $\mathrm{CF}_{4}$ plasma exposure at low bias conditions, the purpose of the amine molecule is to donate electrons to the CNTs. X-ray photoelectron spectroscopy (XPS) and Raman spectroscopy have confirmed both the presence of fluorine and nitrogen on the CNT surface as well as the structural integrity of the CNTs. The functionalisation mechanism was seen to depend on the ion current density and the fluorine plasma during the initial fluorine exposure stages. Electronic characterisation of the plasma fluorinated and the 1,2-diaminoethane functionalised CNTs in backgated geometry was then applied with randomly distributed CNTs on gold electrodes. The fluorinated CNTs have exhibited ptype field dependent behaviour in air, whilst the amine functionalised CNTs have begun to show indication of n-type field dependent behaviour.

To control the positioning of CNTs on a substrate two processes have been developed. The first involves plasma functionalisation method where elemental sulphur and CNTs were heated to $120^{\circ} \mathrm{C}$ in an argon/hydrogen plasma. The sulphur plasma exposed CNTs were observed by XPS to become directly thiolated. Raman spectroscopy again indicated the CNTs had remained intact, although due to the low percentage of sulphur on the CNT surface, $5 \%$, no C-S bonds were observed in the Raman spectra. Thiolated CNTs were then self-assembled across gold electrodes, before being electrically characterised in backgated geometry. Solution properties of the thiolated CNTs limited the reproducibility of the CNT devices, and the yield of self-assembled working devices.

The second process used molecular stamping of 2-thiolpyridine using poly (dimethylsiloxane) (PDMS) stamps; a method which results in a high yield of working CNT devices. Molecular stamping of 2-thiolpyridine using poly(dimethylsiloxane (PDMS) stamping techniques, have been developed to self-assemble CNTs over a substrate and onto predefined electrode structures. By optimising the concentration of 2-thiolpyridine in ethanol and using a dilute suspension of CNTs in 1,2-dichloroethane, CNTs could be self-assembled using two similar fabrication processes. The molecular stamping experiments have confirmed that altering the order of the steps within the fabrication process, to have CNTs on top of electrodes or underneath electrodes, can control the field dependent qualities of devices in a limited gate voltage range. The limiting factor to device reproducibility is the ability to produce homogeneous CNT solutions. With control over the CNT chirality and suspension it is predicted the molecular stamping methods would be a fast and reliable process for high yield CNT devices. 


\section{Declaration of originality}

I hereby declare that the research recorded in this thesis and the thesis itself was composed and originated entirely by myself in the Department of Electronics and Electrical Engineering at The University of Edinburgh.

List your exceptions here and sign before your printed name.

Natalie Plank 


\section{Acknowledgements}

I wish to thank my supervisor and friend Rebecca Cheung for her support and advice over the last 4 years. Without such strong advice and many lengthy discussions this thesis would never have been produced. Also a big thank you for my continued development in particular the support to attend so many interesting conferences and to have the opportunity to study overseas.

Many thanks to Liudi Jiang for friendship, advice and support. I would also like to thank Martin Dicks and Stefan Enderling both for going through it all more or less together and for all the entertaining coffee room discussions. Thanks to Stewart Smith and Jon Terry for sharing their office against their will (and the testlab) and to Tong-Boon Tang for listening.

I would also like to thank those at the SMC who have offered technical advice and support in the cleanroom over the years, in no particular order, Alec Ruthven, Gerry McDade, Ewan McDonald, Andrew Brooke, David Archibald, Kevin Tierney and Andy Bunting. Thanks also to Tom Stevenson and Alan Gundlach for cleanroom training and advice, and to Cameilia Dunare and Bill Parkes for on the spot help and advice.

Thank you to my collaborators in the chemistry department, Robin Andrews, Gavin Forrest and Andrew Alexander. Also to Gavin Whittaker and Gaynor Campbell for use of the plasma etch system, Ian Silverwood and Gordon McDougall for access to FT-IR, and to Francesca Fabianni and Sara Falconi for Raman access. Thank you very much to Ron Brown for the use of the XPS equipment. Thank you also to Anthony Walton, Anthony Snell and Alistair Elfick for academic discussions and general interest in CNTs.

Domo arigatou gozaimasu to all those at NEC fundamental research laboratory in Tsukuba, Japan, Especially to Yudasaka-san for hosting me and to Ishida-san for the many hours in the cleanroom and the advice for CNT experiments. Also to my friends there for the many hours of fun and for taking care of me.

I especially would like to thank Robin Andrews for so many hours talking about nanotechnology and all kinds of experimental and theoretical considerations. And for being 
such a good friend. Thanks also to David Britz for the CNT discussion and surprising friendship.

Thanks to my family, Mum, Dad, Steven and Michael for their encouragement and to Evelyn, Ian and Michelle for all their help over the many years. Lastly I would like to thank all of my friends for listening to me go on about carbon nanotubes over the years and humouring my enthusiasm. A big thank you to Joanne for housing me in my hour of need.

This thesis has been produced using a $\mathrm{bT}_{\mathrm{E}} \mathrm{X}{ }_{\varepsilon}$ Style file provided by George Taylor who can be contacted at: gstaylor@iee.org 


\section{Contents}

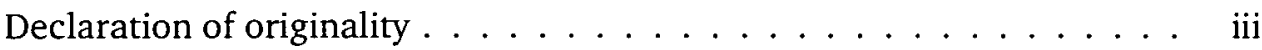

Acknowledgements .................. iv

Contents ......................... vi

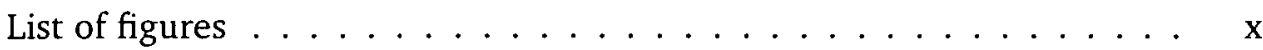

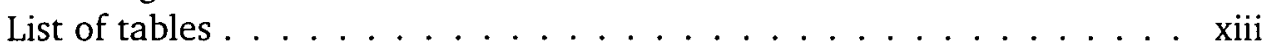

Nomenclature . . . . . . . . . . . . . . . . . xiv

Carbon Nanotube Data and Fundamental Constants . . . . . . . . . . . . xvi

1 Introduction 1

1.1 The Changing Face of Microelectronics . . . . . . . . . . . . . . 1

1.1.1 Why Use Carbon Nanotubes? . . . . . . . . . . . . . . . 2

1.2 Carbon Nanotube Functionalisation . . . . . . . . . . . . 3

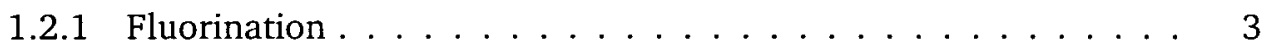

1.2.2 Amine Functionalisation of Carbon Nanotubes for n-type Devices 4

1.3 Self-assembly of Carbon Nanotubes . . . . . . . . . . . . . . . 5

1.3.1 Direct Thiolation of CNTs ................... 5

1.3.2 Molecular Stamping Techniques . . . . . . . . . . . . . . 6

1.4 Thesis Overview . . . . . . . . . . . . . . . . . 7

2 Carbon Nanotubes 10

2.1 The Structure of Carbon Nanotubes . . . . . . . . . . . . . . . . . . . . 10

2.1.1 How the Carbon Nanotube Rolls Up . . . . . . . . . . . . . . . . 12

2.1.2 The Unit Cell, Periodic Boundary Conditions and 1D Energy Dispersions of CNTs . . . . . . . . . . . . . . 13

2.2 Electronic Properties of Carbon Nanotubes . . . . . . . . . . . . . . . . 17

2.2 .1 Metallic CNTs . . . . . . . . . . . . . . . . 17

2.2 .2 Semiconducting CNTs . . . . . . . . . . . . . . 18

2.2.3 Separation of Metallic and Semiconducting CNTs . . . . . . . 19

2.3 Carbon Nanotube Field Effect Transistors . . . . . . . . . . . . . . . . 21

2.3 .1 CNT Doping . . . . . . . . . . . . . . . . . 21

2.3 .2 CNT Logic Devices . . . . . . . . . . . . . . . . . . 23

2.3.3 CNT Electronic Device Summary . . . . . . . . . . . . 23

3 Characterisation Techniques: Spectroscopy and Electronic Device Characterisation

3.1 Raman Spectroscopy . . . . . . . . . . . . . . . . . . . 25

3.1.1 Raman Spectroscopy of Carbon Nanotubes . . . . . . . . . . . . . 28

3.1.2 Raman Spectroscopy: Experimental Considerations . . . . . . . . 30

3.1.3 The Carbon Nanotube Raman Spectra . . . . . . . . . . . . . 30

3.1 .4 Raman Summary . . . . . . . . . . . . . . . . . . 33

3.2 X-ray Photoelectron Spectroscopy (XPS) . . . . . . . . . . . . 33

3.2.1 Deconvolution of the XPS Spectra . . . . . . . . . . 35 
3.2.2 Experimental and Analysis Considerations . . . . . . . . . . 37

3.2 .3 XPS Summary ... . . . . . . . . . . . . . . 37

3.3 Electrical Characterisation . . . . . . . . . . . . . . . . 38

3.3.1 Field Effect Transistors . . . . . . . . . . . . . . . . 38

3.3.2 Metal-Semiconductor Interfaces . . . . . . . . . . . . . 41

3.3 .3 CNT Field Effect Transistors . . . . . . . . . . . . . . . . . 42

3.3.4 Current-Voltage Characteristics ... . . . . . . . . . . 44

3.3.5 Schottky Diode Characterisation ... . . . . . . . . . 45

3.3.6 Field Effect Transistor Characterisation . . . . . . . . . . . . 46

3.3.7 Electronic Device Characterisation Summary . . . . . . . . . . . 49

3.4 Characterisation Techniques: Conclusion . . . . . . . . . . . . . . 49

4 Plasma Diagnostics and Microfabrication Techniques $\quad 50$

4.1 Plasma Diagnostics . . . . . . . . . . . . . . . . . . . 50

4.1.1 The Langmuir Probe . . . . . . . . . . . . . . . 53

4.1 .2 Optical Emission Spectroscopy . . . . . . . . . . . . . 54

4.1.3 Plasma Diagnostics: Summary . . . . . . . . . . . . . . 55

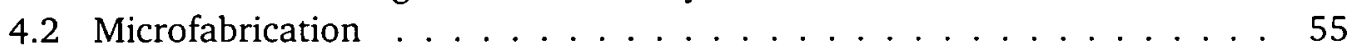

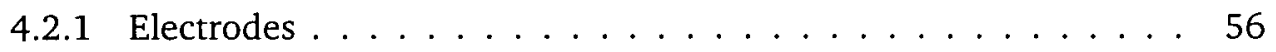

4.2 .2 PDMs Stamps . . . . . . . . . . . . . . . 6 60

4.2.3 Microfabrication Techniques: Summary . . . . . . . . . . 61

5 Plasma Fluorination of Carbon Nanotubes $\quad 62$

5.1 The Fluorination of Carbon Nanotubes, a Brief Review . . . . . . . . . 62

5.1.1 Plasma Fluorination of Carbon Nanotubes . . . . . . . . . . . 63

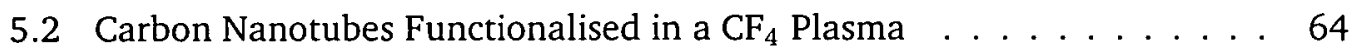

5.2.1 Experimental Setup of Fluorinated Plasmas . . . . . . . . . . . 64

5.2.2 $\mathrm{CF}_{4}$ Plasma Exposed CNTs: The Raman Spectra . . . . . . . . . . 64

5.2 .3 The $\mathrm{D} / \mathrm{G}$ Ratio . . . . . . . . . . . . . . . . . 65

5.2.4 Scanning Electron Microscopy of the CNT Surface . . . . . . . . 66

5.2.5 $\mathrm{CF}_{4}$ Plasma Exposed CNTs: The XPS Spectra . . . . . . . . . . 66

5.2.6 Deconvolution of the XPS Spectra: The C1s Region . . . . . . . 67

5.2.7 Deconvolution of the XPS Spectra: The F1s Region . . . . . . . 68

5.2.8 The Semi-ionic and Covalent F1s Regions . . . . . . . . . . 69

5.2.9 Summary: $\mathrm{CF}_{4}$ Plasma Functionalisation . . . . . . . . . . 70

5.3 The Comparison of $\mathrm{CF}_{4}$ and $\mathrm{SF}_{6}$ Plasma . . . . . . . . . 71

5.3.1 $\mathrm{CF}_{4}$ and $\mathrm{SF}_{6}$ Plasma Exposed CNTs: The XPS Spectra . . . . . . 71

5.3.2 $\mathrm{CF}_{4}$ and $\mathrm{SF}_{6}$ Plasma Exposed CNTs: The Attachment Mechanism 72

5.3.3 The Position of the Fls Peak . . . . . . . . . . . . 77

5.3.4 Transmission Electron Microscopy of the CNTs Surface . . . . . 79

5.3.5 Summary of $\mathrm{CF}_{4}$ and $\mathrm{SF}_{6}$ Plasma Exposed CNTs and the Attachment Mechanism . . . . . . . . . . . . . . 80

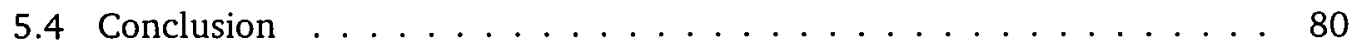

6 Diamine Functionalisation of Plasma Fluorinated Carbon Nanotubes $\quad 83$

6.1 N-type Doping of CNTs, a Brief Review . . . . . . . . . . . . . . . 83

6.2 Experimental Method of the F-tube Diamine Functionalisation . . . . . 84 
6.3 Functionalised CNTs Spectroscopy . . . . . . . . . . . . . . . . 86

6.3.1 Atomic Ratios on the CNT Surfaces . . . . . . . . . . . . 86

6.3 .2 The C1s Region . . . . . . . . . . . . . . . . . 88

6.3 .3 The F1s Region . . . . . . . . . . . . . . . . . . . . 89

6.3 .4 The $\mathrm{N} 1 \mathrm{~s}$ Region . . . . . . . . . . . . . . . . . . . . . . . . . . . . . . . .

6.3 .5 The Raman Spectra . . . . . . . . . . . . . . . . . . . . . . . . . . . . . .

6.4 The Diamine Functionalisation Mechanism . . . . . . . . . . . . . . . . . 92

6.4.1 Summary of the Diamine Functionalisation . . . . . . . . . . 93

6.5 Electronic Characterisation of F-CNTs and N-CNTs . . . . . . . . . . . . 94

6.5.1 The Experimental Method . . . . . . . . . . . . . . . . . 94

6.5.2 Source-Drain Electronic Behaviour of F-CNTs . . . . . . . . . . 996

6.5.3 Source-Drain Electronic Behaviour of N-CNTs . . . . . . . . . 998 98

6.5.4 Gate field dependent Electronic Behaviour of F-CNTs and N-CNTs 99

6.5 .5 N-type Doping of CNTs . . . . . . . . . . . . . . . . . 100

6.5 .6 Device Characterisation . . . . . . . . . . . . . 101

6.5.7 Summary of the I-V characteristics . . . . . . . . . . . . . 102

6.6 Conclusion . . . . . . . . . . . . . . . . . 103

7 Plasma Thiolation of Carbon Nanotubes for Self Assembly 105

7.1 Thiolation of Carbon Nanotubes, a Brief Review . . . . . . . . . . . . . . 105

7.2 Sulphur Plasma Exposure of CNTs . . . . . . . . . . . . . . . . 106

7.2.1 Experimental procedure . . . . . . . . . . . . . 106

7.2 .2 The XPS Spectra of Thiolated CNTs . . . . . . . . . . . 108

7.2.3 The Raman Spectra of Thiolated CNTs . . . . . . . . . . . 110

7.2 .4 Direct Thiolation Summary . . . . . . . . . . . . . . . 112

7.3 Self-assembly of Thiolated Carbon Nanotubes . . . . . . . . . . . . 112

7.3.1 Self-Assembly Experimental Procedure . . . . . . . . . . . . 112

7.3.2 Scanning Electron Microscopy . . . . . . . . . . . . . . . . . 113

7.3.3 Self-Assembly Summary . . . . . . . . . . . . . . . . . . . 117

7.4 Electrical Characterisation of Plasma Thiolated Carbon Nanotubes . . . . 117

7.4.1 Electronic Testing: Experimental Procedure . . . . . . . . . . 117

7.4.2 Thiolated CNT Bulk Film Behaviour . . . . . . . . . . . . . . . 117

7.4.3 Back-gated Thiolated CNT Device Behaviour . . . . . . . . . . . . 118

7.4 .4 Electronic Characterisation Summary . . . . . . . . . . . . 120

7.5 Conclusion . . . . . . . . . . . . . . . . . . . 120

8 Molecular Stamping to Position Carbon Nanotubes 122

8.1 Molecular Stamping and Self-Assembly Techniques, a Brief Review . . 122

8.2 Molecular Stamping for CNT Devices . . . . . . . . . . . . . . . . 123

8.2.1 Molecular Stamping Experimental Procedure . . . . . . . . . . 123

8.2.2 The Molecular Stamping Mechanism . . . . . . . . . . . . . 126

8.2.3 PDMS Stamp Geometry: Large Stamps . . . . . . . . . . . . . . . 128

8.2.4 PDMS Stamp Geometry: Small Stamps . . . . . . . . . . . . . . 130

8.2 .5 Molecular Stamping Summary . . . . . . . . . . . . . . 132

8.3 Electronic Characterisation . . . . . . . . . . . . . . . 133

8.3.1 Source-Drain Characteristics of Self-Assembled CNTs . . . . . . 133

8.3.2 Gate Field Dependent Characteristics of Self-Assembled CNTs . . 134 
8.4 Comparison of Field-Dependent Behaviour between Method I and Method

II Type Devices . . . . . . . . . . . . . . . . . . . . . 136

8.4.1 The Device Structure . . . . . . . . . . . . . . . . 136

8.4 .2 Gate Screening Effects . . . . . . . . . . . . . . . . . 138

8.5 Characterisation of Method I Field Dependent Devices . . . . . . . . 139

8.5.1 Subthreshold Characteristics . . . . . . . . . . . . . . . . . . 139

8.5.2 Electronic Characterisation Summary . . . . . . . . . . . . . . 141

8.6 Statistical Differences in the CNT Devices . . . . . . . . . . . . . . 141

8.7 Conclusion . . . . . . . . . . . . . . . . . . 143

9 Conclusion $\quad \mathbf{1 4 5}$

9.1 Plasma Fluorination of Carbon Nanotubes . . . . . . . . . . . . . . 145

9.2 Amine Functionalisation of Fluorinated Carbon Nanotubes . . . . . . . 146

9.3 Direct Thiolation of Carbon Nanotubes . . . . . . . . . . . . . . . . . 147

9.4 Molecular Stamping, for Carbon Nanotubes Device Fabrication . . . . . . 148

9.5 Further Work . . . . . . . . . . . . . . . . . . 150

$\begin{array}{lr}\text { A Papers } & 152\end{array}$

$\begin{array}{ll}\text { References } & 164\end{array}$ 


\section{List of figures}

1.1 . The roadmap for gate scaling. . . . . . . . . . . . . 2

2.1 A schematic representation of the energy levels of carbon . . . . . . . . 11

2.2 The $\pi$ bonding of carbon in the CNT sheet. . . . . . . . . . . . 11

2.3 A representation of how a carbon nanotube rolls up. . . . . . . . . . . 12

2.4 The three different types of carbon nanotubes. . . . . . . . . . . . . . 13

2.5 Part of the unit cell and extended Brillouin zone of armchair and zig zag carbon nanotubes . . . . . . . . . . . . . . . . . 14

2.6 The allowed wave vectors in the first Brillouin zone for both an armchair and a zig zag CNT. . . . . . . . . . . . . . . . 15

2.7 The $1 \mathrm{D}$ energy dispersion relations for CNTs. . . . . . . . . . . . . 16

2.8 The experimental evidence for coloumb blockade. . . . . . . . . . . . 17

2.9 The conduction across a kink in a CNT. . . . . . . . . . . . . . 20

$2.10 \mathrm{n}$-type field effect devices made by vacuum annealing and chemical doping. 22

3.1 The vibrations of the radial breathing mode (RBM) and the G-band. . . . 29

3.2 An example of the Raman setup used. . . . . . . . . . . . . . 30

3.3 An example of the typical Raman spectra of CNTs. . . . . . . . . . . . . . 31

3.4 An example of the Raman spectra of CNTs functionalised using an aggressive acid. . . . . . . . . . . . . . . . . 32

3.5 An example of the photoelectron effect . . . . . . . . . . . . 34

3.6 An example of the F1S peak, fitted to a) Semi-ionic and covalent fluorine plus background effects, and b) multiple Gaussian peaks at $1 \mathrm{eV}$ spacing. 36

3.7 Schematic of a standard FET and a CNT FET . . . . . . . . . . . . . . 39

3.8 The output for both a p-type and n-type FET in enhancement mode. . . . 40

3.9 For a n-type semiconductor making contact with a metal . . . . . . . . 41

3.10 Current transport mechanisms. . . . . . . . . . . . . . . . . 42

3.11 The band diagrams of the CNT FET . . . . . . . . . . . . 43

3.12 The CNT FET probe setup . . . . . . . . . . . . . . . 45

4.1 A schematic overview of a parallel plate reactor. . . . . . . . . . . . . . 51

4.2 A typical example of a Langmuir probe J-V characteristic. . . . . . . . . . . 54

4.3 An example of the optical emission spectra observed. . . . . . . . . . 55

4.4 The schematic process flow for the lithographic patterning of a substrate 57

4.5 The schematic process flow for metalisation . . . . . . . . . . . . 59

4.6 The schematic formation of a PDMS stamp . . . . . . . . . . . . 60

5.1 The Raman spectra of a typical control and a typical $\mathrm{CF}_{4}$ plasma exposed CNT samples ... . . . . . . . . . . . . . . . 6 65

5.2 The ratio of the $\mathrm{D}$-band to the G-band as a function of $\mathrm{CF}_{4}$ plasma exposure conditions ......................... 66

5.3 SEM images of typical control and $\mathrm{CF}_{4}$ plasma exposed samples . . . . . 67 
5.4 The C1s peaks of the control sample and the $\mathrm{CF}_{4}$ plasma exposed sample 68

5.5 The $\mathrm{F} 1 \mathrm{~s}$ peaks of the control sample and the $\mathrm{CF}_{4}$ plasma exposed sample

5.6 Typical $\mathrm{F} 1 \mathrm{~s}$ peaks of the control, $\mathrm{CF}_{4}$ exposed and $\mathrm{SF}_{6}$ exposed samples

5.7 The plasma conditions as measured by OES and LP plasma diagnostics as a function of D.C. bias voltage . . . . . . . . . . . . . 74

5.8 The surface characteristics of the CNT bucky papers as a function of bias voltage . . . . . . . . . . . . . . . . . 76

5.9 The F1s peaks for altered plasma feedstock gases . . . . . . . . . . 78

5.10 TEM images of a) pristine tubes, b) $\mathrm{CF}_{4}$ plasma exposed samples and c) $\mathrm{SF}_{6}$ plasma exposed samples . . . . . . . . . . . . . . 79

6.1 Schematic representation of the amine-functionalisation process . . . . 85

6.2 The surface characteristics of the functionalised CNTs as a function of plasma exposure time . . . . . . . . . . . . . 87

6.3 The C1s spectra of the F-CNTs and the N-CNTs . . . . . . . . . . . . . . 89

6.4 A typical F1s spectra of the functionalised CNTs . . . . . . . . . . . . 90

6.5 The diamine functionalisation as a function of initial $\mathrm{F} / \mathrm{C}$ ratio . . . . . . 91

6.6 The typical Raman spectra from the pristine and functionalised CNTs. . . 92

6.7 Schematic representation of the device setup . . . . . . . . . . . . . . 94

6.8 The SEM images of randomly distributed functionalised HiPCO CNTs between electrodes . . . . . . . . . . . . . . . . 95

6.9 The source-drain characteristics for all F-CNTs devices at $\mathrm{Vg}=0 \mathrm{~V} \ldots . .97$

6.10 The source-drain characteristics for all N-CNTs devices at Vg $=0 \mathrm{~V}$. . . 98

6.11 A logarithmic plot of the drain current vs source drain voltage for the F-CNTs and N-CNTs devices . . . . . . . . . . . . . . . 102

7.1 A schematic representation of the plasma thiolation set-up . . . . . . 107

7.2 The S2p peaks of the control CNTs, sulphur/CNTs and sulphur/CNTs exposed to $\mathrm{Ar} / \mathrm{H}_{2}$ plasma . . . . . . . . . . . . . . . . 108

7.3 The influence of the $\mathrm{H}_{2}$ flow rate in the $\mathrm{Ar} / \mathrm{H}_{2}$ plasma exposure on (a) the $\mathrm{S} / \mathrm{C}$ ratio and (b) the position of the S2p energy peak of the sulphur/CNTs109

7.4 The Raman spectra of control CNTs, sulphur/CNTs and sulphur/CNTs exposed to $\mathrm{Ar} / \mathrm{H}_{2}$ plasma . . . . . . . . . . . . . . . . . 111

7.5 Examples of HiPCO plasma thiolated carbon nanotubes bridging Au electrodes . . . . . . . . . . . . . . . . . . . . 114

7.6 Examples of laser ablation plasma thiolated carbon nanotubes bridging Au electrodes . . . . . . . . . . . . . . . . . . . 115

7.7 An SEM image of the sulphur/CNTs exposed to $\mathrm{Ar} / \mathrm{H}_{2}$ self assembled across gold trenches . . . . . . . . . . . . . . 116

7.8 The source-drain characteristics of a self-assembled S-CNTs bundle produced at room temperature, and a randomly dispersed bundle of pristine CNTs. . . . . . . . . . . . . . . . . . . . . 118

7.9 I-V characteristics of back-gated self-assembled thiolated carbon nanotubes119

8.1 A schematic representation of the molecular stamping process . . . . . 124

8.2 The vacuum chuck . . . . . . . . . . . . . . . 126 
8.3 A schematic representation of the 2-thiolpyridine and CNT self-assembly process . . . . . . . . . . . . . . . . . . . 127

8.4 A schematic representation of the course alignment stamp and electrode geometry ... . . . . . . . . . . . . . 128

8.5 The SEM image of a large bundle of CNTs and the measured source-drain characteristics . . . . . . . . . . . . . . . . 128

8.6 SEM image of a bundle of CNTs with a junction. Corresponding sourcedrain characteristics are shown. . . . . . . . . . . . . . . . . . . 129

8.7 The I-V and SEM image of a small bundle of CNTs clearly showing a junction . . . . . . . . . . . . . . . . . . . . 129

8.8 A schematic representation of the fine alignment and electrode geometry 130

8.9 SEM image of CNT device made via method I . . . . . . . . . . . . . 131

8.10 SEM image of CNT device made via method II . . . . . . . . . . . . . . 132

8.11 The source-drain characteristics for devices produced by method I and II 134

8.12 The backgated output characteristics for devices produced by method I and II . . . . . . . . . . . . . . . . . . . . 135

8.13 The device structures . . . . . . . . . . . . . . . . . . . . 137

8.14 The gate dependence of a device made by method II in comparison to a device made by drop-coating of CNTs . . . . . . . . . . . . . . . . . 138

8.15 The backgated output characteristics for devices method I (a), method I (b), method I (c) and method II . . . . . . . . . . . . . . . . . 139

8.16 Logarithmic backgated output characteristics for devices produced by method I and the corresponding subthreshold slope dependence on Vds 140

8.17 Gate field dependent conduction of four devices made by method I (b) . 142

A.1 Reprint of APL vol 83, p2462 . . . . . . . . . . . . . . . 153

A.2 Reprint of APL vol 83, p2463 . . . . . . . . . . . . . . . . . 154

A.3 Reprint of APL vol 83, p2464 . . . . . . . . . . . . . . . . . 155

A.4 Reprint of Microelectronic engineering vol 73-74, p578 . . . . . . . 156

A.5 Reprint of Microelectronic engineering vol 73-74, p579 . . . . . . . . . . 157

A.6 Reprint of Microelectronic engineering vol 73-74, p580 . . . . . . . . . 158

A.7 Reprint of Microelectronic engineering vol 73-74, p581 . . . . . . . . 159

A.8 Reprint of Microelectronic engineering vol 73-74, p582 . . . . . . . . 160

A.9 Reprint of APL vol 85, p3229 . . . . . . . . . . . . . . 161

A.10 Reprint of APL vol 85, p3230 . . . . . . . . . . . . . . . 162

A.11 Reprint of APL vol 85, p3231 . . . . . . . . . . . . . 163 


\section{List of tables}

3.1 Raman scattering outcomes . . . . . . . . . . . . . . 27

$5.1 \mathrm{I}_{\text {semi-ionic }} / \mathrm{I}_{\text {covalent }}$ intensity ratio for the $\mathrm{C}-\mathrm{F}$ bonds $\ldots \ldots \ldots . \ldots 70$

5.2 Reactor conditions of the Vacutec RIE system. . . . . . . . . . . . . 72

5.3 Position of the F1s peak. *The CNTs are placed on a conducting substrate. 79

6.1 The plasma exposure conditions and the resulting stoichiometry of the CNTs post functionalisation . . . . . . . . . . . . 86

6.2 Standard probe station sweep conditions for backgated CNT devices . 96

7.1 The reactor conditions. The reactor was heated to $120^{\circ} \mathrm{C}$, with R.F. power $250 \mathrm{~W}$ and plasma exposure time of 1 minute . . . . . . . . . . 107

8.1 The self-assembly fabrication process . . . . . . . . . . 125 


\section{Nomenclature}

\begin{tabular}{|c|c|c|}
\hline Name & Symbol & Unit \\
\hline Current & I & A \\
\hline Voltage & $\mathrm{V}$ & $\mathrm{V}$ \\
\hline Induced dipole moment & $\mu$ & $\mathrm{Am}^{2}$ \\
\hline Electric field & $\vec{E}$ & $\mathrm{Vm}^{-1}$ \\
\hline Polarisability & $\alpha$ & $\frac{A m^{2}}{V}$ \\
\hline Vibrational frequency & $\nu_{v i b}$ & $s^{-1}$ \\
\hline Frequency & $\nu$ & $s^{-1}$ \\
\hline Time & $\mathrm{t}$ & $s$ \\
\hline Energy & $\mathbf{E}$ & $\mathrm{eV}$, or $\mathrm{J}$ \\
\hline Wavenumber & $\omega$ & $c m^{-1}$ \\
\hline Binding energy & $\mathrm{BE}$ & $\mathrm{eV}$ \\
\hline Kinetic energy & $\mathrm{E}_{K E}$ & $\mathrm{eV}$ \\
\hline Ion current density & Ii & $\mathrm{Acm}^{-2}$ \\
\hline Work function of a metal & $\phi_{m}$ & $\mathrm{eV}$ \\
\hline Work function of a semiconductor & $\phi_{s}$ & $\mathrm{eV}$ \\
\hline Barrier height & $\phi_{b}$ & $\mathrm{eV}$ \\
\hline Barrier height for a n-type semiconductor & $\phi_{b n}$ & $\mathrm{eV}$ \\
\hline Electron affinity & $x$ & $\mathrm{eV}$ \\
\hline Valence band energy & $\mathrm{E}_{v}$ & $\mathrm{eV}$ \\
\hline Conduction band energy & $\mathrm{E}_{c}$ & $\mathrm{eV}$ \\
\hline Fermi Level energy & $\mathrm{E}_{F}$ & $\mathrm{eV}$ \\
\hline Drop in potential due to interface & $\mathrm{V}_{b i}$ & $\mathrm{~V}$ \\
\hline Diffusion voltage (band bending) & $\mathrm{V}_{d}$ & V \\
\hline Applied source bias & $\mathrm{V}_{d s}$ & $\mathrm{~V}$ \\
\hline Applied gate bias & $\mathrm{V}_{g}$ & $\mathrm{~V}$ \\
\hline Measured drain current & $\mathrm{I}_{d s}$ & A \\
\hline Gate source voltage & $V_{G S}$ & $\mathrm{~V}$ \\
\hline Threshold voltage & $\mathrm{V}_{t h}$ & $\mathrm{~V}$ \\
\hline
\end{tabular}




\begin{tabular}{|c|c|c|}
\hline Name & Symbol & Unit \\
\hline Ideality factor & $\eta$ & \\
\hline Temperature & $\mathrm{T}$ & ${ }^{\circ} \mathrm{C}$ or $\mathrm{K}$ \\
\hline Saturation current & $I_{s t}$ & A \\
\hline Diode contact area & $S$ & $\mathrm{~cm}^{2}$ \\
\hline Subthreshold slope & $S$ & $\mathrm{~V} / \mathrm{dec}$ \\
\hline Carrier mobility & $\mu$ & $\frac{c m^{2}}{V s}$ \\
\hline Conductivity & $\sigma$ & $\mathrm{S} / \mathrm{cm}$ or $\Omega^{-1} / \mathrm{cm}^{-1}$ \\
\hline Conductance & G & $S$ or $\Omega^{-1}$ \\
\hline Gate capacitance & $c_{g}$ & $\mathrm{~F} / \mathrm{cm}^{2}$ \\
\hline Plasma potential & $\mathrm{V}_{P}$ & V \\
\hline Ion current density & $\mathrm{I}_{i}$ & $\mathrm{~A} / \mathrm{cm}^{2}$ \\
\hline Standard cubic centimetre & & $\mathrm{sccm}$ \\
\hline Chiral vector & $C_{h}$ & $\mathrm{~nm}$ \\
\hline CNT diamter & $d_{t}$ & $\mathrm{~nm}$ \\
\hline Translational vector & $\mathbf{T}$ & $\mathrm{nm}$ \\
\hline
\end{tabular}

Abbreviations

Single wall carbon nanotube

CNT or SWNT

$\mathrm{X}$-ray photoelectron spectroscopy

XPS

Optical emission spectrscopy

OES

Langmuir probe

Scanning electron micsroscopy

SEM

Transmission electron microscopy

TEM

Atomic force microscopy

AFM

Poly(dimethylsiloxane)

PDMS

Field effect transistor

FET

Reactive ion etcher

RIE

Polytetrafluoroethylene

PTFE

Isopropanol 


\section{Carbon Nanotube Data and Fundamental Constants}

\begin{tabular}{lcr}
\hline Properties of Carbon Nanotubes & Symbol & Value \\
\hline Average diameter distribution & $d_{t}$ & $0.8-1.4 \mathrm{~nm}$ \\
Carbon-carbon bond length & C-C & $1.44 \AA$ \\
Lattice constant & a & $1.44 \times \sqrt{3}=2.49 \AA$ \\
Carbon-carbon tight binding overlap & $\gamma_{o}$ & $2.5 \mathrm{eV}$ \\
Conductance quantization & $G$ & $n \times(12.9 k \Omega)^{-1}$ or $\frac{2 e}{h}$ \\
Resistivity & $\rho$ & $10^{-4} \Omega-\mathrm{cm}$ \\
Maximum current density & $J$ & $10^{13} \mathrm{~A} / \mathrm{m}^{2}$ \\
Richardson constant & $\mathrm{A}$ & 129 \\
\hline
\end{tabular}

\begin{tabular}{lcr}
\hline Fundamental Constant & Symbol & Value \\
\hline Planck's constant & $\mathrm{h}$ & $6.672 \times 10^{-34} J_{S}$ \\
Elementary charge & e or $q$ & $1.6 \times 10^{-19} \mathrm{C}$ \\
Boltzmann's constant & $k_{B}$ & $1.38 \times 10^{-23} \mathrm{JK}^{-1}$ \\
\hline
\end{tabular}


"For a successful technology, reality must take precedence over public relations, for Nature cannot be fooled".

\section{Richard Feynman}




\section{Chapter 1 Introduction}

The introductory chapter will introduce the reader as to why there is a need for materials such as carbon nanotubes in the microelectronics industry. The methods applied in this investigation will be laid out along with a brief explanation of the current work in the field. The aim is to familiarise the reader with the motivation behind the work carried out here.

Finally an overview of the thesis structure will be explained chapter by chapter, to allow the reader fast access to required information.

\subsection{The Changing Face of Microelectronics}

Since the prediction made by Moore in 1965 that the number of transistors on a chip roughly doubles every two years, Moore's Law [1], the microelectronics industry has innovated and developed technologies to meet these targets. Fundamental research carried out both by industry and academia over the years has proved to live up to expectations and demands for faster, more powerful and efficient microchips. The silicon technology road map has been accurately predicting the device scales to be achieved over the last few decades. However, looking at the predictions made for the gate node dimensions in 2003 in figure 1.1, it can be seen that the limits of silicon technology will soon be reached.

The patterning of nanometer scaled silicon gates is a huge technological challenge. Not only this, the fundamental physics dominating the transport properties of semiconducting materials at such a scale will become dominated by quantum effects. Researchers are now looking towards novel material to become the semiconducting materials of future devices. One such promising materials are carbon nanotubes. 


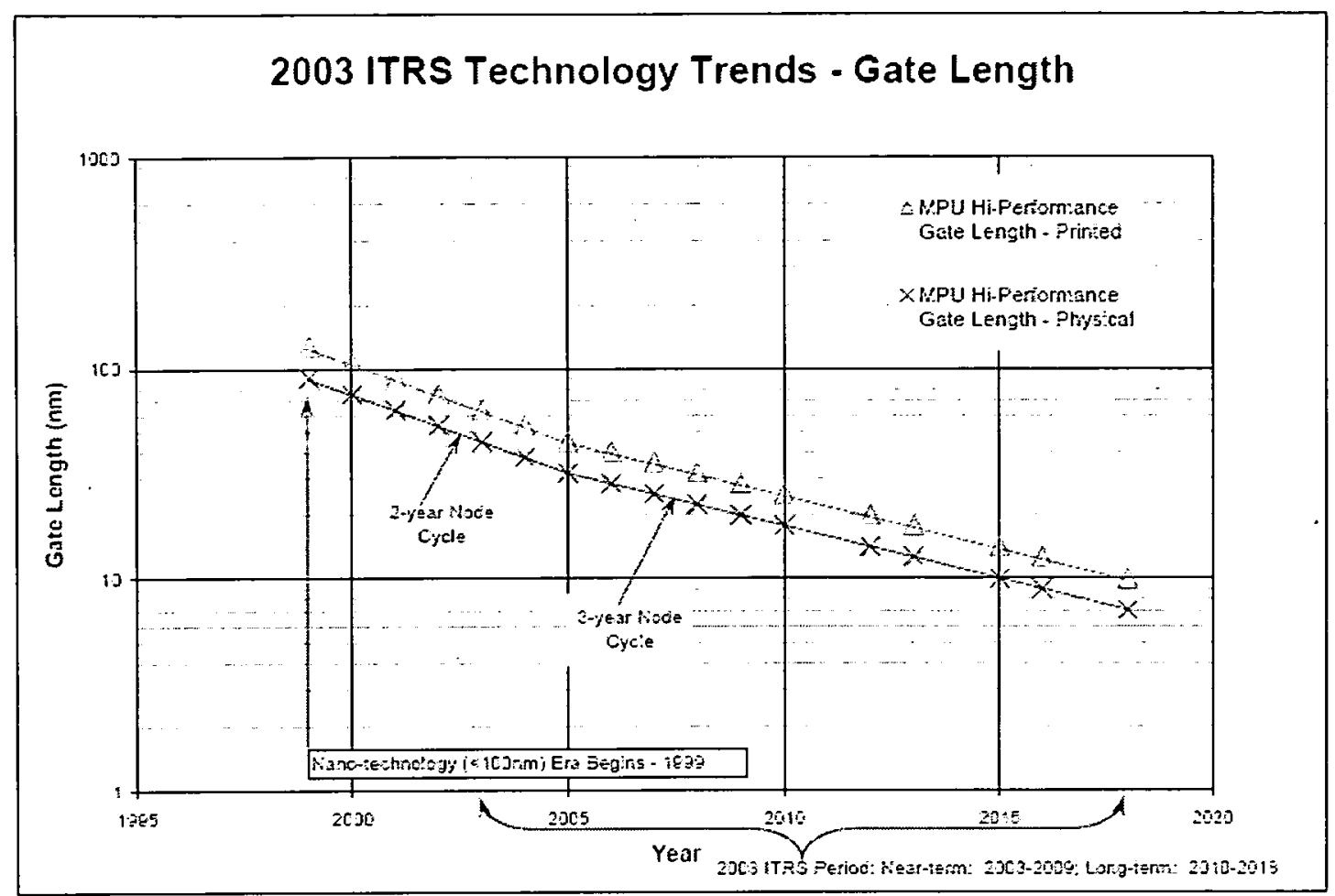

Figure 1.1: The silicon technology roadmap predictions for transistor gate node scaling.

\subsubsection{Why Use Carbon Nanotubes?}

Carbon nanotubes (CNTs) have caught the imagination of scientist and engineers over a wide and diverse range of disciplines due to their unique electronic and physical properties. Not only do CNTs have the ability to conduct as a metal or a semiconductor, they have also shown exceptional tensile strength, high thermal conductivity and all whilst being inherently on the nanoscale [2]. The CNTs themselves can be simply thought of as a sheet of graphite rolled up into a tube. The way in which the tube has rolled up then determines many of the properties of the CNT, fundamentally for this investigation, the electrical characteristics of the CNTs.

Due to the fascinating nature of the CNT material, not only for fundamental science, but also for multiple device applications a great deal of hype has surrounded CNT technologies. By 2000 there were review articles in popular science magazines such as Physics World [3] where single nanotube electronics could be reviewed and summarised to the date. There were also numerous articles in the popular press such as the Economist [4] 
and the Financial Times [5] heralding nanotechnology as a good investment area with carbon nanotube characteristics leading the way as potential future materials. Even tabloid and local newspapers have featured articles on carbon nanotubes [6].

The speed at which CNT electronics was advancing was in large part due to the new improvements in the growth and manufacturability of the CNTs. The high pressure carbon dioxide (HiPCO) CNT growth process developed at Rice university and sold under the company name CNI [7], had lead to a high yield single walled carbon nanotube growth process. Although the growth of carbon nanotubes is still a constantly evolving field of active research, the growth methods and mechanism will not be discussed in this thesis, as no growth experiments were carried out. All CNT electronic characterisation was done on commercially available tubes and those from collaborators. The growth details of which will be referenced as necessary.

\subsection{Carbon Nanotube Functionalisation}

As discussed above, it is well known that CNTs can behave as metallic conductors or semi-conductors depending upon the physical structure of the tubes. However, although the CNTs exhibit these advantageous electronic properties several problems remain with the material for electronic device applications. To begin with the inherent electronic behaviour of CNT field effect transistors (CNT FETs) in air is p-type, limiting their uses for logic applications. Also the CNTs have a tendency to bundle together as ropes.

As the CNTs are initially chemically inert a first point for CNT electronics can be to make the CNTs chemically reactive by means of functionalisation. The benefits of the functionalisation processes are two-fold. The general increased reactivity of the CNTs can be exploited to further functionalise CNTs to alter the electronic behaviour by chemical doping, or more simply to increase the solubility of the CNTs for uniform solutions.

\subsubsection{Fluorination}

The initial methods employed throughout this work have been to functionalise the normally unreactive single walled carbon nanotubes (CNTs, or SWNT throughout this 
work) by fluorination. The advantages of fluorinating CNTs are many-fold $[8,9]$. In particular an increased reactivity acts as a staring point for further reactions, however, reactivity also improves suspensions, allowing CNTs to be more easily dispersed and therefore isolated. However, previous fluorination of CNTs, largely developed by Mickelson et al. [10] at Rice University, had involved long exposures to elemental fluorine gas diluted by argon at elevated temperatures. These processes were time consuming and often resulted in the loss of CNT structure.

The plasma functionalisation methods developed here, exploited short functionalisation times and low damage conditions to fluorinate the CNTs. Other research groups have also used similar plasma fluorination methods of CNT functionalisation after the development of the work done here [11-14]. The fluorination method was observed to effectively functionalise the CNT sidewalls without damaging the CNT structure.

Importantly, the fluorination is used as a starting point of various derivisations by nucleophilic substitutions with a view of controlling the electronic properties of the CNTs from p-type to n-type behaving devices.

\subsubsection{Amine Functionalisation of Carbon Nanotubes for n-type Devices}

The plasma fluorinated CNTs made during this work have been further functionalised with amine containing groups. Amine functionalisation is proposed to donate electrons to the CNTs, thus producing n-type CNT devices.

The use of amine containing groups to alter the inherently p-type SWNTs to n-type devices has been exploited by several groups due to the amines electron donating nature. The use of polyethylene imine (PEI) first carried out by Dai et al. [15] provided permanently n-type doped SWNT devices.

In this study the substitution of 1,2-diaminoethane has been employed, a modified version of the reaction developed by Stevens et al. [16], to dope F-SWNTs. The FSWNTs were prepared by $\mathrm{CF}_{4}$ plasma exposure as discussed elsewhere $[17,18]$ and in Section 5.3.1 where the fluorination would lead to both semi-ionic and covalent fluorine attached to the sidewalls of the SWNTs.

The resulting CNT FETs devices exhibited n-type field dependent behaviour due to elec- 
tron donation from the amine molecule.

\subsection{Self-assembly of Carbon Nanotubes}

As well as the inherent p-type behaviour of the CNT devices there is also a problem with the CNT material for electronic device applications in the selective positioning of CNTs onto prefabricated electrodes. One method explored in the literature has been to grow CNTs on predefined points across two metallic regions acting as electrodes [1926]. Another method is to position electrodes onto CNTs which have been deposited onto a substrate of some kind [27-30]. Here, the ultimate goal is to position carbon nanotubes without altering the electronic properties of the CNTs, by exploiting means of self-assembling chemical reactions to position the carbon nanotubes onto predefined electrodes.

There are a number of reasons for choosing this route. Firstly, controlling the growth position of carbon nanotubes is a successful method of producing carbon nanotubes with reliable positional accuracy. However, the processes involved in the growth of carbon nanotubes often require high temperatures [31,32], not suitable for integration into current silicon technology fabrication processes. Secondly, a metallic catalyst is required in most of the well known growth methods. Once growth is complete there is no clear process for removing the catalyst particles, which may in turn affect the ultimate behavioural properties of the CNT devices.

The need to accurately position carbon nanotubes on a large scale basis has been addressed by various groups using different methods used in the selective positioning of carbon nanotubes. Thiolation of CNTs through wet chemical techniques had been used to self-assemble CNTs onto gold [33-35]. Other methods for positioning CNTs have included DNA hybridisation [36] Langmuir-Blodgett films [37] and polar molecules [38].

\subsubsection{Direct Thiolation of CNTs}

To take advantage of the thiol gold self-assembly, CNTs had been previously thiolated by substitution reactions. In all cases CNTs were firstly functionalised by long acid purification and cutting steps to obtain carboxylic acids $[33-35,39]$. Smalley et al. have func- 
tionalised the CNTs with a long chain carbon group to produce CNT- $\left(\mathrm{CH}_{2}\right)_{11}-\mathrm{SH}$ [33], whilst Liu et al. produced CNT-CONH- $\left(\mathrm{CH}_{2}\right)_{2}-\mathrm{SH}$ [34]. A shorter chain reaction was then developed by Lim et al. [35] where via several acid reflux steps, conversion of carboxyl groups to hydroxyl groups, further conversion of hydroxyl groups to chloride groups, and finally thiolation, $\mathrm{CNT}-\mathrm{CH}_{2}-\mathrm{SH}$ tubes were produced.

Here, CNTs were directly functionalised in a plasma containing sulphur and hydrogen to produce a thiol ( $\mathrm{SH}$ ) bond on the CNT sidewalls, known as thiolated CNTs. The selfassembly between gold and $\mathrm{SH}$ is a well known phenomenon [40-45] and by selectively patterning gold electrodes the thiolated CNTs can assemble across electrode structures.

\subsubsection{Molecular Stamping Techniques}

Also, of particular interest in comparison to the process developed here, was the selective positioning of CNTs employed by Rao et al. and published in Nature [38] using polar molecules and surface passivation. Two separate regions of polar and non-polar molecules were fabricated using some form of standard self-assembly procedure (PDMS stamping or dip-pen lithography). Between the polar and non-polar regions the $\mathrm{SiO}_{2}$ substrate surface had been passivated using a silane based material. The CNTs were then observed to attach across the polar regions bridging the silane passivated surface.

Here, the process developed has been greatly simplified, whereby CNTs were positioned using soft-lithography inking techniques $[40,41]$. By stamping 2-thiolpyridine a nonpolar and short chain R-thiol molecule, a room temperature two step fabrication process was developed, whereby the thiol end of the molecule selectively adhered to the gold or oxide surface, whilst the pyridine end of the molecule attracted the CNT sidewalls.

Both the direct thiolation and the molecular stamping techniques resulted in CNTs which could be back-gated for field dependent device characterisation. The working device yield for the direct thiolation method was relatively poor at around $10 \%$, whilst the molecular stamping method produces a much higher working device yield of $90 \%$. 


\subsection{Thesis Overview}

The main body of this work deals with the physical alteration of CNTs by means of chemical functionalisation. Chemical functionalisation processes were achieved for both doping and selective positioning of carbon nanotubes. Molecular stamping techniques have also been applied to position carbon nanotubes, a process potentially applicable to both pristine and doped CNTs.

The structure of the thesis is divided to enable the reader quick access to the relevant results, a brief summary of each chapter is made below.

Chapter 2: Carbon Nanotubes. A description of carbon nanotubes, including their structure and predicted electronic behaviour has been made. The theory surrounding the physical description of carbon nanotubes is described. The reader will then be introduced to the highlights of the field of CNT electronic devices. A brief review of the experimentally realised electronic devices made to date is given.

Chapter 3: Characterisation Techniques, Spectroscopy and Electronic Device Characterisation. The spectroscopic techniques of $\mathrm{x}$-ray photoelectron spectroscopy (XPS) and Raman spectroscopy, required to analyse the functionalisation processes carried out on the CNTs are described. The previous experimental work in particular on CNT Raman structure is reviewed. The experimental setup along with practical advice and considerations discovered for the spectroscopy of CNT samples are discussed.

The principles of electronic characterisation of nanoelectronic devices are set out including a comparison to well-known silicon based electronic structures such as field effect transistors and Schottky diodes. An introduction into microelectronic probing techniques specific to the CNT devices fabricated is then made.

Chapter 4: Plasma Diagnostics and Microfabrication Techniques. A description of plasma diagnostic techniques necessary to determine the plasma functionalisation mechanism for the CNT fluorination is made. These include optical emission spectroscopy and actinometrical calculations, along with Langmuir probe techniques.

Current microelectronic fabrication processes which are essential in the production of any electrode structures and PDMS print masters are also explained. 
Chapter 5: Plasma Fluorination of Carbon Nanotubes. The experimental chapters then begin with the plasma fluorination of carbon nanotubes. The spectroscopic characterisation results of the fluorinated CNTs is made with Raman and XPS. The CNTs are also inspected using electron microscopy.

The reaction mechanisms of the plasma fluorination process are studied by use of plasma diagnostic tools and CNT surface characterisation. By comparison of a $\mathrm{SF}_{6}$ and $\mathrm{CF}_{4}$ plasma feedstock gas, the C-F bond on functionalised CNTs is discussed.

Chapter 5: Amine Functionalisation of Plasma Fluorinated Carbon Nanotubes. The further functionalisation of $\mathrm{CF}_{4}$ plasma fluorinated CNTs by amine functionalisation is described. The functionalisation process is characterised by Raman and XPS and the CNTs are shown to remain intact. The amine functionalisation of the CNTs is seen to depend directly on the initial fluorination.

A conversion of CNT devices from p-type to n-type by amine functionalisation is observed. The electrical characterisation of the functionalised CNTs are then discussed.

Chapter 7: Plasma Thiolation of Carbon Nanotubes for Self-Assembly. The third results chapter details the thiolation of CNTs using a novel plasma process. Spectroscopic surface characterisation of the CNTs is again applied using Raman and XPS. The result have shown that low levels of sulphur in the thiol region have bonded to the CNTs without damaging the CNT structure.

The subsequent self-assembly of the thiolated CNTs was observed using SEM. The CNTs were observed to bridge narrow electrode structures. Where bridging was successful, the electrical behaviour of the CNTs could be probed. The device yield and behaviour is discussed.

Chapter 8: Molecular Stamping to Position Carbon Nanotubes. Finally, in the fourth results chapter, the fabrication of Poly(dimethylsiloxane) (PDMS) stamps and molecular stamping techniques for CNT positioning is discussed. The molecular stamping of 2 thiolpyridine is considered as a means of realising a large scale fabrication process for high yield post-growth CNT devices. The self-assembly of CNTs was characterised by SEM.

The electronic behaviour of the CNT devices are then characterised in back-gated ge- 
ometry. The field dependent behaviour is highlighted and comparison to other devices is made.

Chapter 9: Conclusion. In the conclusion the contribution of this work to the field of carbon nanotube electronics is expressed and the required work for future development is discussed.

The highlights of the research have been published in peer reviewed journals or presented at relevant international conferences showing that the work has been a contribution to the field of carbon nanotube electronics. All publications made as a direct result of the work produced here are reprinted in the Appendices A. 


\section{Chapter 2 Carbon Nanotubes}

To begin to understand the properties of carbon nanotubes it is first necessary to consider the physical structure of carbon nanotubes. The following chapter explains the theoretical description of the CNTs structure and the subsequent electronic behaviour. The carbon nanotubes can behave as either metallic or semiconductors depending on how the tube has formed. The theory behind the electronic behaviour of the CNTs is described briefly. Experimentally achieved CNT electronic devices will then be considered, both as nanoscale metallic conductors and as field dependent semiconductor devices.

\subsection{The Structure of Carbon Nanotubes}

Carbon is an interesting and versatile element which forms the basis of organic chemistry. It is recommended that for a full appreciation of the versatility of carbon based chemistry, the reader consults an organic chemistry textbook [46]. A carbon atom has six electrons available and will fill the lower energy level shells first in accordance to the aufbau principle. Firstly there are 2 electrons in the $1 \mathrm{~s}$ shell and this is the case for all carbon hybridisations. The remaining 4 electrons, the valence electrons, are then split between the $2 \mathrm{~s}$ orbital and the $2 \mathrm{p}_{x}, 2 \mathrm{p}_{y}$ and $2 \mathrm{p}_{z}$ orbitals as shown in figure 2.1 (a).

However, carbon is available in several different forms making it such a versatile building block for organic chemistry. The different forms of carbon are most usefully considered in terms of their hybridisations, $\mathrm{sp}, \mathrm{sp}^{2}$ and $\mathrm{sp}^{3}$. In the case of $\mathrm{sp}$ hybridisation one of the $2 \mathrm{p}$ orbitals is mixed with the $2 \mathrm{~s}$ figure $2.1(\mathrm{~b})$. For the $\mathrm{sp}^{2}$ hybridisation two of the $2 p$ orbitals are mixed with the $2 \mathrm{~s}$, figure $2.1(\mathrm{c})$ and it is this hybridisation of carbon which gives us graphite, $\mathrm{C}_{60}$ and other fullerenes including CNTs. In the case of $\mathrm{sp}^{3}$ hybridisation, responsible for carbon in the form of diamond, all 4 electrons are in equal energy shells as effectively the $2 \mathrm{~s}$ is raised and the three $2 \mathrm{p}$ orbitals are lowered, figure 2.1(d). 


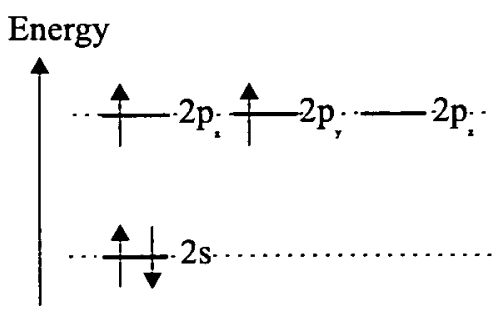

(a) The outer energy shells of carbon

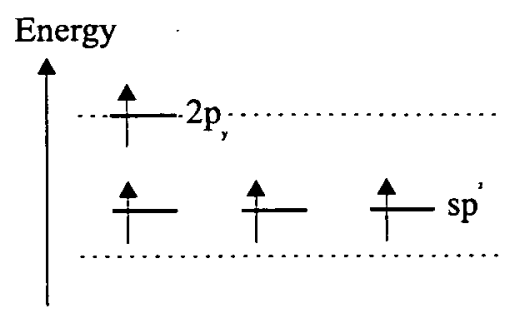

(c) Carbon $\mathrm{sp}^{2}$ hybridisation

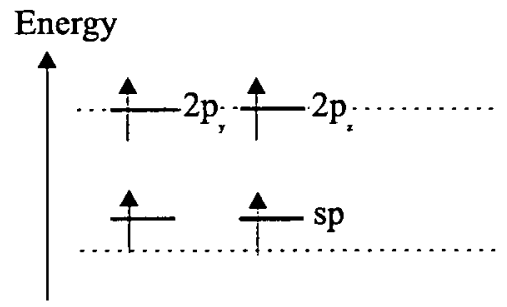

(b) Carbon sp hybridisation

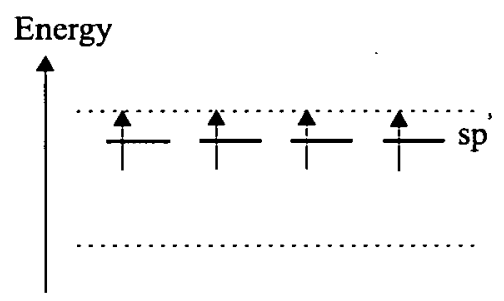

(d) Carbon $\mathrm{sp}^{3}$ hybridisation

Figure 2.1: A schematic representation of the energy levels of carbon

Graphite, carbon with $\mathrm{sp}^{2}$ hybridisation, forms a hexagon based structure as shown in figure 2.3 will be the only form of carbon further considered here. Each $\mathrm{sp}^{2}$ carbon atom is capable of forming 3 sigma $(\sigma)$ bonds with the nearest neighbour carbon and 1 pi $(\pi)$ bond, perpendicular to the hexagonal carbon plane. A $\sigma$ bond is strong and corresponds to the head on overlap of two atomic orbitals, such as a covalent bond, these $\sigma$ bonds play no role in the conductivity of carbon graphite [47]. The $\pi$ bond is considerably weaker. However, it is this weak $\pi$ bond which allows one free electron per unit cell to contribute to the conduction of graphite between stacked layers of graphite sheets [47], shown in figure 2.2 .

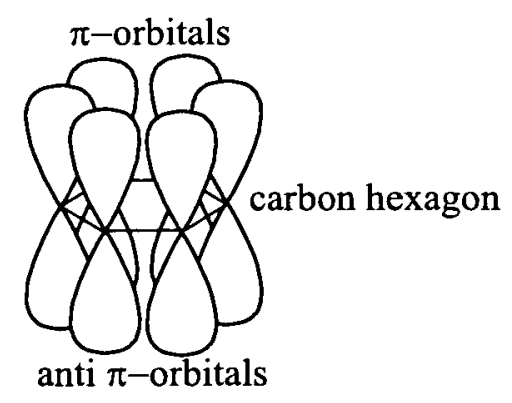

Figure 2.2: The $\pi$ bonding of carbon in the CNT sheet. The $\sigma$ bonds are in the plane of the carbon hexagon. 


\subsubsection{How the Carbon Nanotube Rolls Up}

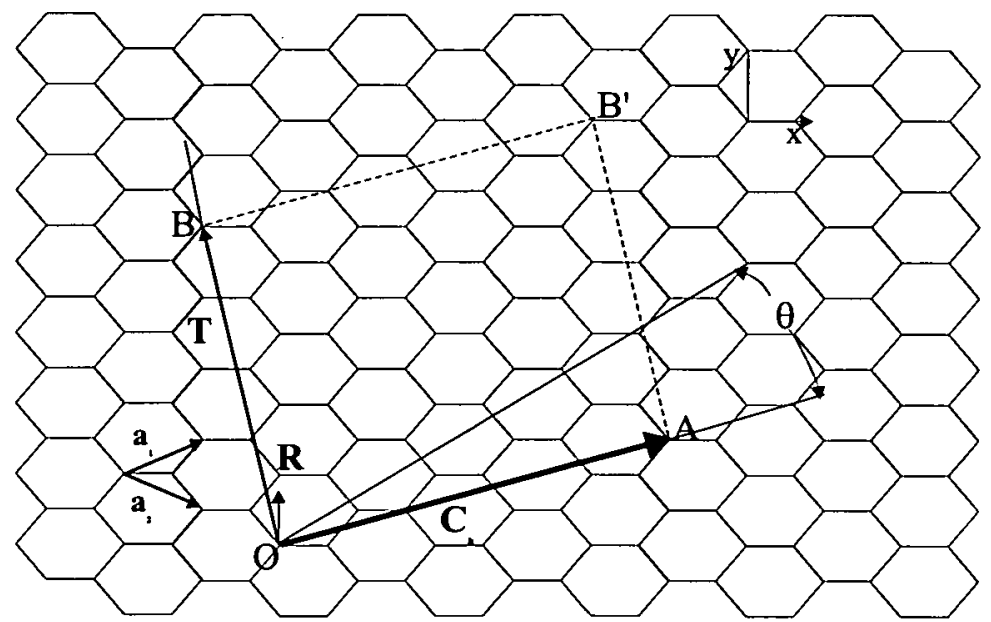

Figure 2.3: A representation of how a carbon nanotube rolls up. Sites $O$ and $A$ and $B$ and $B$ ' meet seamlessly to form a nanotube, recreated from figures by Dresselhaus et al in [2]. The representation shown here indicates a $(4,1)$ CNT.

A carbon nanotube can be described as a sheet of $\mathrm{sp}^{2}$ carbon which has been rolled up along the chiral vector $C_{h}$ equation 2.1, as shown in figure 2.3. Important theoretical work on the understanding of the CNTs physical and electronic behaviour has been developed using the tight binding model ${ }^{1}$ of graphite [47] and imposing periodic boundary conditions due to the rolling up of the tube. Work led by White [49], Hamada [50] and Dresselhaus $[51,52]$ all provided important contributions to the theoretical understanding of the CNT structure. Although this is not an exhaustive list of those whom have worked on the field, these papers cover the discussion presented here as a general overview of the CNT theoretical behaviour. How the tube has rolled up along its axis determines many of the electrical properties of the CNTs and can be described by the $(n, m)$ indices defined by the chiral vector $C_{\boldsymbol{h}}$, also known as the Hamada vector.

$$
C_{h}=a_{1} n+a_{2} m \equiv(n, m)
$$

The Hamada vector also leads to the powerful deduction of the CNT tube diameter, $d$, where $a=1.44 \times \sqrt{3}=2.49 \AA$ is the lattice constant.

\footnotetext{
${ }^{1}$ The tight binding model is explained in solid state physics textbooks, such as that by Kittel [48] and in application to CNTs by the introductory Carbon Nanotube textbook by Dresselhaus et al. [2]
} 


$$
d=\frac{C_{h}}{\pi}=\frac{a \sqrt{n^{2}+n m+m^{2}}}{\pi}
$$

The $(n, m)$ indices of the CNTs then determine the electronic properties of the nanotubes and can be split up into three main types, $(n, n)$ arm chair tubes where $\theta= \pm 30^{\circ}$, $(n, 0)$ zig zag tubes where $\theta=0^{\circ}$ and for all other $(n, m)$ chiral tubes. The three main tube structures are shown in figure 2.4. Importantly, where $(n-m)=3 p$, the tubes are metallic, and if $(n-m)=3 p \pm 1$, the tubes are semiconducting, where $p=$ integer, leading to all $(n, n)$ (armchair) tubes being metallic. The $(n, 0)$ (zig zag) tubes are semiconducting when $n \neq$ integer multiple of 3 . When $n=$ integer multiple of 3 however, the CNT will behave as a small gap semiconductor, and at room temperature this gap is so small that the tubes behave as a metal $[50,51,53]$ for all practical purposes.

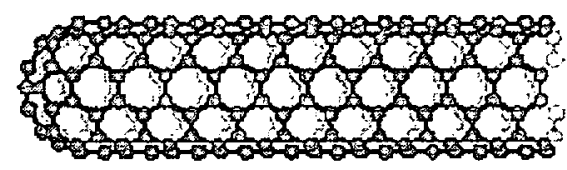

(a) An armchair CNT $(n, n)$
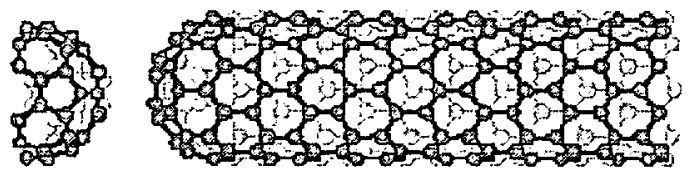

(b) A zig zag CNT $(n, 0)$

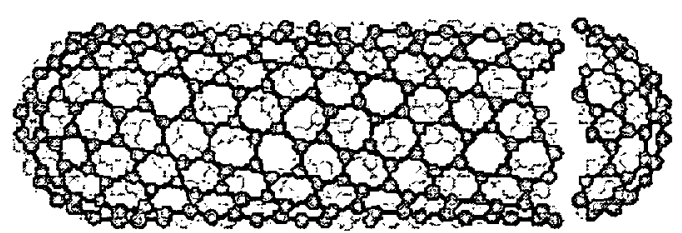

(c) A chiral CNT $(n, m)$

Figure 2.4: The three different types of carbon nanotubes. When $(n-m)=3 p$, the tubes are metallic, and if $(n-m)=3 p \pm 1$, the tubes are semiconducting, where $p=$ integer. Taken from [2]

\subsubsection{The Unit Cell, Periodic Boundary Conditions and 1D Energy Disper- sions of CNTs}

The unit cell for a carbon nanotube is given by the rectangle formed by the chiral vector $C_{h}$ and the translational vector $T, O A B B^{\prime}$ as in figure 2.3 [2]. The unit cell and extended Brillouin zones for armchair and zig zag CNTs are shown in figure 2.5, again reconstructed from the diagrams presented by the Dresselhaus group [2,52].

The CNTs are interesting due to their one dimensional (1D) periodic structure along 

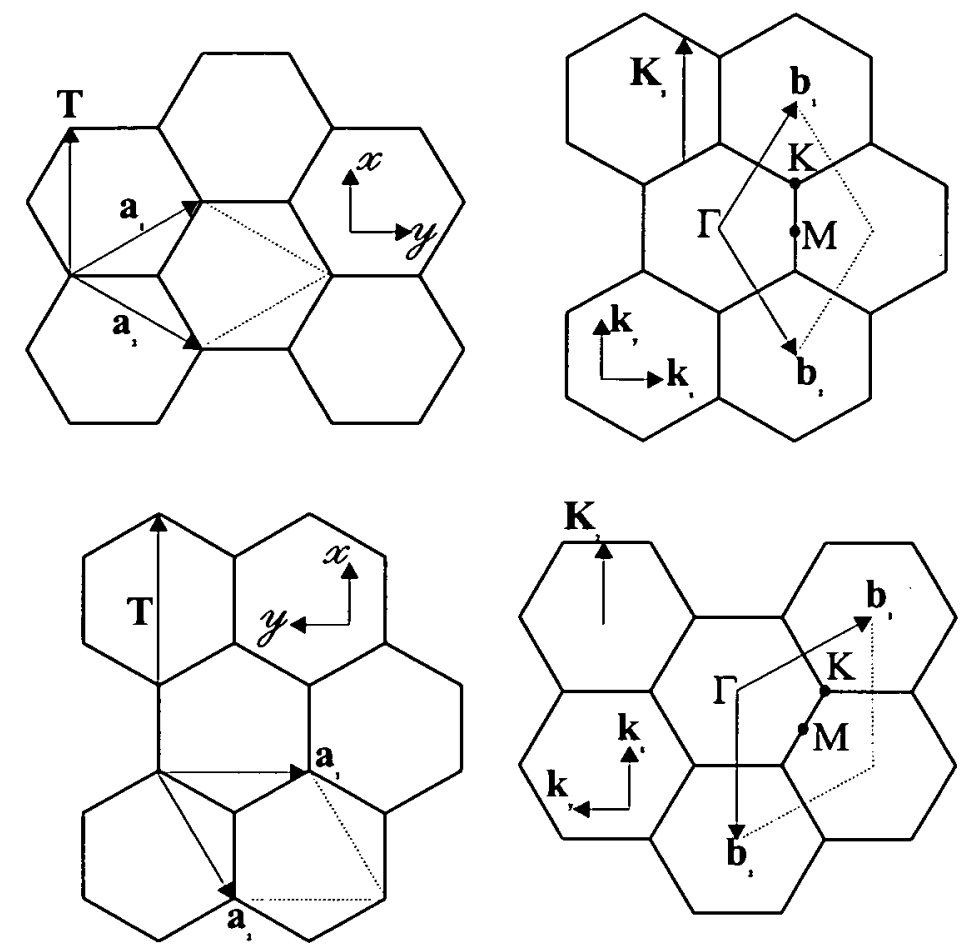

Figure 2.5: Part of the unit cell and extended Brillouin zone of armchair (top) and zig zag (bottom) carbon nanotubes, taken from $[2,52]$. The dashed lines show the zones for graphite as a comparison [47].

the direction of the tube axis [51]. In the direction of the circumference, it is necessary to apply periodic boundary conditions due to the bending of the graphite sheet in the tube structure. The boundary conditions $C_{\boldsymbol{h}} \cdot \boldsymbol{k}=2 \pi m$ are applied to eliminate regions from the two-dimensional (2D) energy dispersion relations of the $\pi$ bands of graphite, necessary due to the rolling up of the graphite sheet to make the CNT structure. In doing so the $1 \mathrm{D}$ energy bands can be obtained by slicing of the $2 \mathrm{D}$ energy dispersion regions in the direction of $C_{h} \cdot k$.

Using the unit cells, figure 2.5 and applying the periodic boundary conditions to the CNTs the allowed wave vectors determine the electronic behaviour of the CNTs. The allowed wave vectors for both a metallic and semiconducting CNT are shown in figure 2.6. Where the allowed wave vectors include the $K$ point the CNT will behave as a metal, and where it does not as a semiconductor $[50-52,54]$.

A plot of the $1 \mathrm{D}$ energy dispersion relations for both metallic and semiconducting CNTs is shown in figure 2.7. The cross over of the $1 \mathrm{D}$ energy dispersion relations at the 


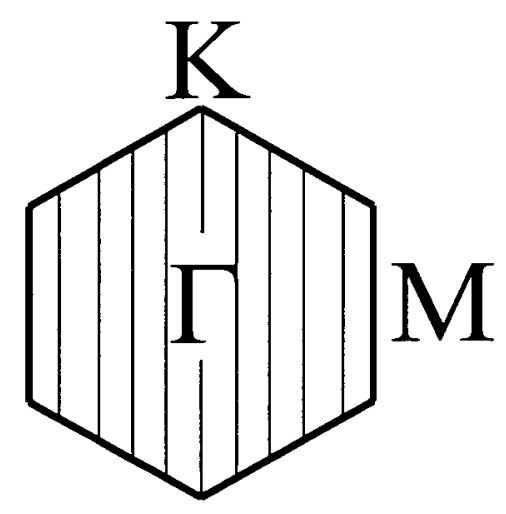

(a) The allowed wave vectors slicing through the first Brillouin zone of an armchair CNT. As the wave vectors cut though the $\mathrm{k}$ points, the tube behaves as a metallic conductor.

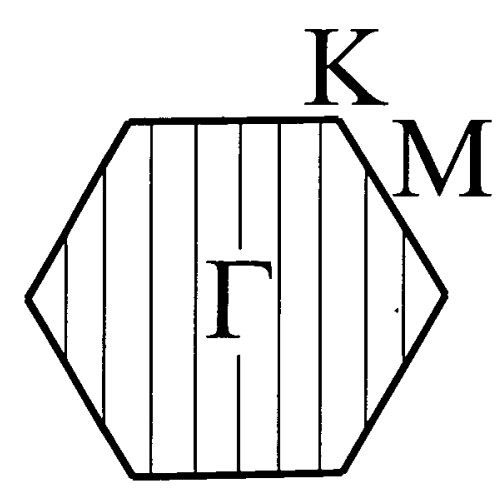

(b) The allowed wave vectors slicing through the first Brillouin zone of a zig zag CNT. As the wave vectors do not cut though the $K$ points, the tube behaves as a semiconductor.

Figure 2.6: The allowed wave vectors in the first Brillouin zone for both an armchair and a zig zag CNT. Recreated from Hamada et al [50].

Fermi level $\left(\mathrm{E}_{F}\right)$ signifies a metallic conductor, figure 2.7(a) whilst the presence of a gap indicates a semiconductor, figure $2.7(\mathrm{~b})$. When the semiconductor gap is very small the CNT will behave as a metal at room temperature, figure 2.7(c). The 1D energy dispersion relations show clearly how the tube rolling effects and the allowed wavevectors can alter the CNT electronic behaviour.

Further discussion of the CNT physical structure will be made in the Raman spectroscopy Chapter 3.1. In the meantime it is important to note that these theoretical predictions of the CNT structure have been verified by experimental evidence in Raman spectroscopy, as will be discussed, Chapter 3.1. 


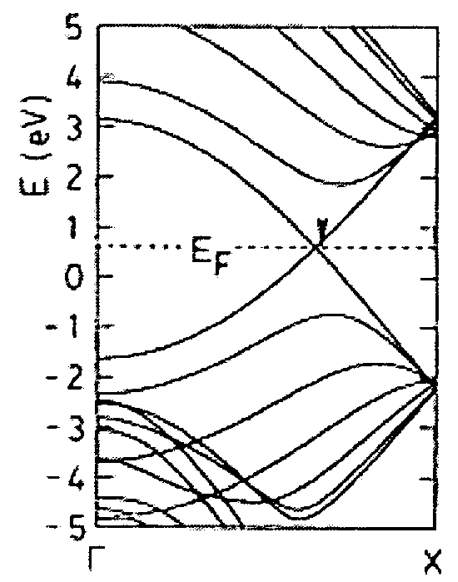

(a) The $1 \mathrm{D}$ energy dispersion relations for a armchair tube.

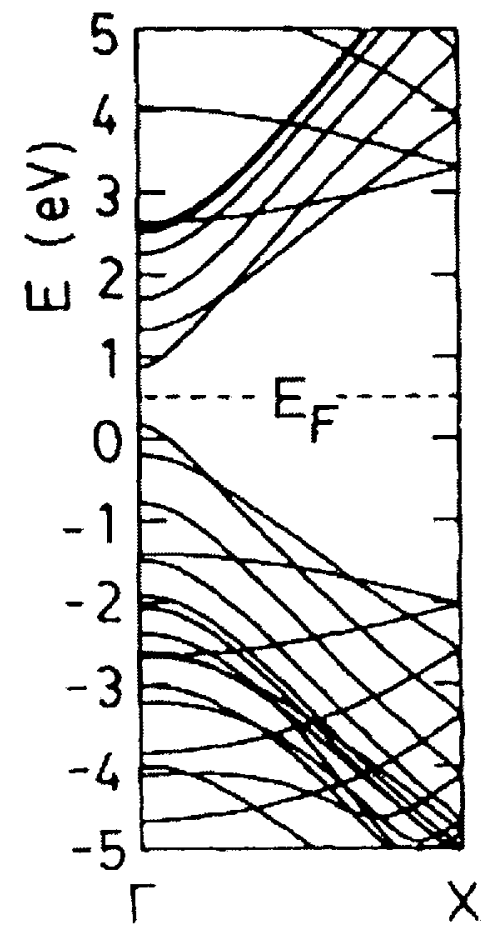

(b) The 1D energy dispersion relations for a large bandgap zigzag tube

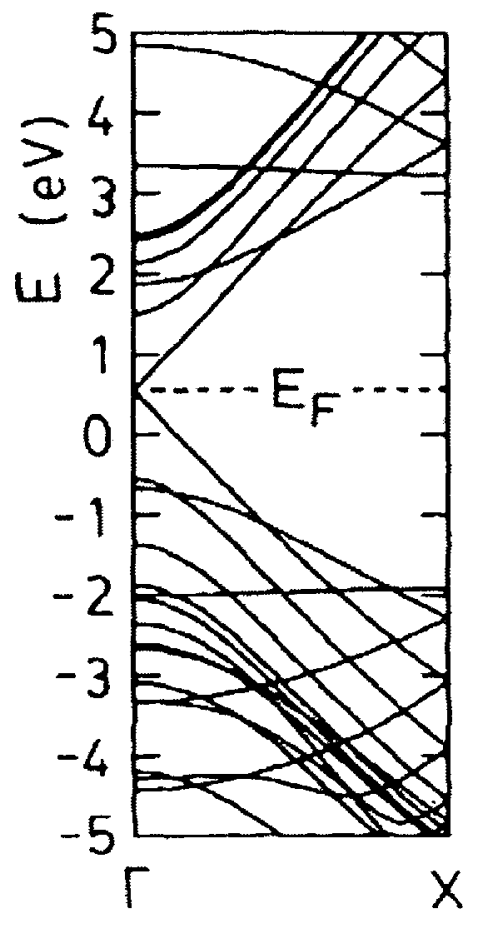

(c) The 1D energy dispersion relations for a small band gap zigzag tube

Figure 2.7: The 1D energy dispersion relations for CNTs taken from Hamada et al [50]. 


\subsection{Electronic Properties of Carbon Nanotubes}

The surprising electrical behaviour of CNTs as either metallic or semiconducting 1D nanoscale conductors, predicted by the tight binding model, has indeed been observed in experimental investigations.

\subsubsection{Metallic CNTs}

Metallic single walled carbon nanotubes (CNTs) were seen to act as genuine quantum wires in 1997 under the direction of Cees Dekker [55]. These were the first measurements of individual CNTs, and the devices were prepared by drop-coating laser ablation CNTs onto predefined Pt electrodes. Electrical conduction was seen to occur though discrete electron states and coloumb charging was observed by taking measurements at temperatures as low as $4 \mathrm{~K}$.

It was becoming apparent that the behaviour of CNT metallic type devices was close to ballistic transport $[56,57]$. Experiments carried out have shown that palladium electrodes make ohmic contact to CNTs [58-60] and evidence of the CNTs behaving as a Luttinger liquid and displaying coloumb oscillations have been seen [61-63].

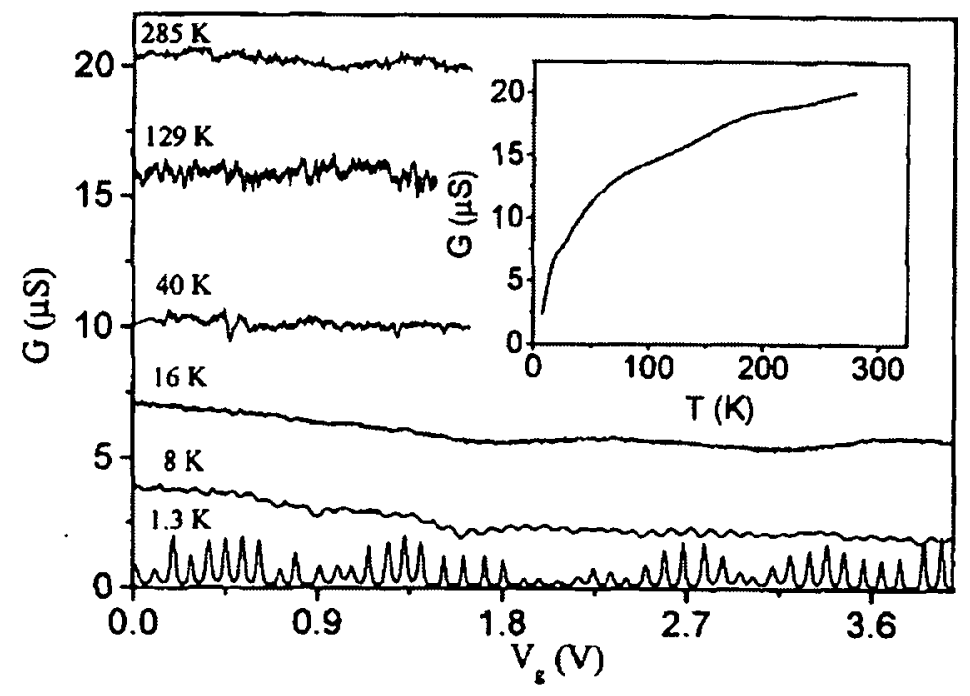

Figure 2.8: The experimental evidence for coloumb blockade and CNT luttinger liquid behaviour. Taken from [61]

Figure 2.8 shows a reproduction of the early I-V data collected from metallic CNTs by Paul McEuen's group [61]. The coloumb blockade effects become visible at $1.3 \mathrm{~K}$ and 
the conductance is seen to rise exponentially with increasing temperature as is the case for a Luttinger liquid.

In the practical realisation of CNT devices, the electronic transport was often hindered by the CNT/electrode interface. The quality of the nanotube/electrode interface was seen to be determined by the choice of metal used for the electrode. Initially it was presented that the work function of the metal could determine the nature of the contact. For example, a metal with a work function higher than that of the CNT (4.5 eV [64]) should produce an ohmic contact. In the case of palladium, with work function $5.1 \mathrm{eV}$, ohmic contacts were observed. When the work function of palladium was decreased by hydrogen exposure the contacts became less reliable and the ohmic nature was lost. However, in the case of platinum, work function $5.7 \mathrm{eV}$ [65], the contacts are not ohmic. The effect is further confused by the evidence showing that some titanium (work function $4.1 \mathrm{eV}$ [65]) CNT contacts have been ohmic [59], although also often Schottky.

The Ohmic nature of the contacts is therefore deduced to be a combination of metal work function and the wettability of the metal allowing a good contact to be made. In the case of metallic CNT devices, the reduction of contact resistance has allowed ballistic transport to be observed. In the case of semiconducting nanotubes the effect of the CNT/electrode interface is also important. The majority of discussion on observed nanotube electronic behaviour will focus on semiconducting CNT characteristics as the primary aim of these investigations has been to produce a fabrication process for reliable CNT field effect transistor devices.

\subsubsection{Semiconducting CNTs}

In 1998 the Cees Dekker group at Delft characterised CNT field effect transistors [66] as their fabrication process had led to both metallic and semiconducting CNT devices on a single chip. In the initial stages of CNT FET research many compared the CNT devices to the standard silicon MOSFET (metal oxide semiconductor field effect transistor). For a MOSFET, the gate dependent conduction is due to channel conduction effects and depends on the level of dopants in the device. Further experimental characterisation of CNT FET devices was later carried out largely by the group at IBM, which showed that CNT FETs are Schottky barrier transistors $[29,64,67-71]$ and not analogous to the 
MOSFET model. However, this is not an exclusive field, and many other papers have appeared on the field effect behaviour of CNT FETs [2].

The confirmation of the Schottky barrier type transistor theory for CNT FETs, came when it was observed that switching the source and drain electrode on a particular CNT FET device led to altered field-dependent characteristics [68]. The Schottky barrier CNT FETs in contrast to the MOSFET model previously used, are not affected by the carriers already present and instead depend on the band bending at the Schottky barrier created at the CNT/electrode interface, induced by the gate field $[68,70,72]$. Such a description holds true for short channel.CNT FETs [53], where conduction is dominated by ballistic transport. However, long channel CNT FETs do depend on the carriers [53, 73] and parameters such as mobility can be extracted from the transport characteristics, which will be discussed later in Section 2.3 .

As well as using CNTs as the channel in field effect devices or as metallic interconnects, it is also possible to produce unique devices by joining two CNTs together or making a kink in the CNT tube structure. The combination of metallic and semiconducting CNTs as a single junction device has been predicted to act as a rectifier [54]. Indeed even along a single nanotube probed by STM the I-V character along a single tube was seen to alter, most likely due to defects in the CNT tube structure [74]. The defect sites, most likely $(5,7)$ pentagon-heptagon sites on a tube would be equivalent to the junction formed by 2 CNTs bridging together.

Figure 2.9 shows the effect that a kink on a CNT can have on the CNTs conduction. Across the kink the CNT is seen to act as a rectifier, whilst in the region above the kink ohmic conduction is observed. Such altering device behaviour dependent upon the positioning of the electrodes along the CNTs provides an interesting basis for CNT device fabrication. By placing multiple electrodes along a CNT differing device characteristics can be obtained.

\subsubsection{Separation of Metallic and Semiconducting CNTs}

Although the potential applications as field effect devices, interconnects and novel rectifiers have been demonstrated experimentally, many problems arise with the tendency for CNTs to bundle together in mixtures of metallic and semiconducting CNTs. There is 


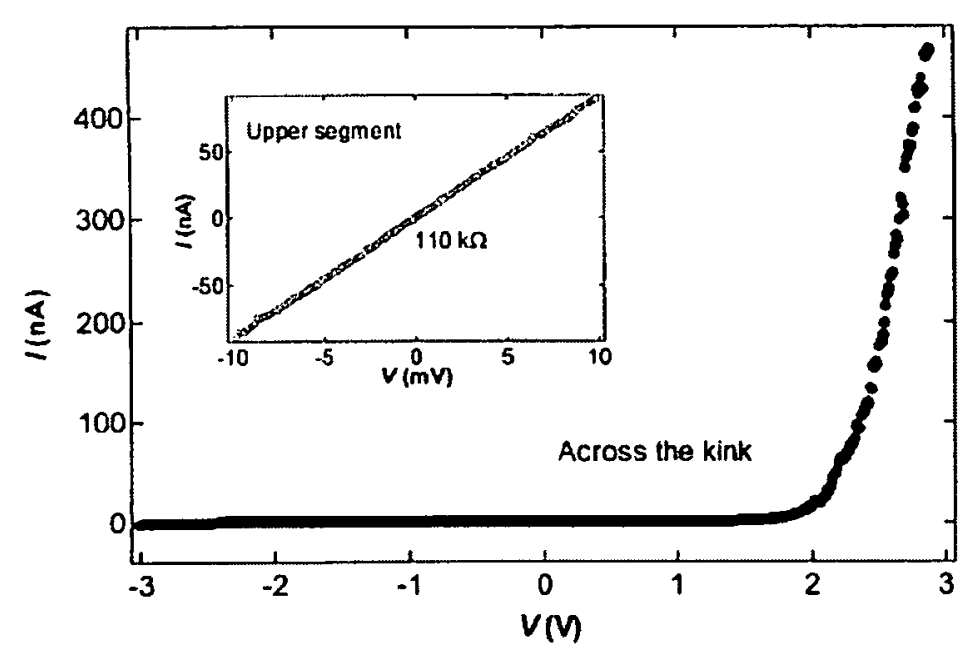

Figure 2.9: The conduction across a kink in a CNT taken from [54]. Above the kink ohmic conduction is observed, whilst across the kink rectifying behaviour can be seen.

as yet no clear way to produce purely metallic or purely semiconducting CNTs, although laser ablation does tend towards more armchair CNTs $[55,74]$ and some CVD growth conditions towards semiconducting $[60,75,76]$.

Certain techniques have been applied for the selective removal of metallic CNTs from mixed bundles. Firstly there is a technique known as burn off, whereby in a bundle of CNTs attached to electrodes the semi-conducting tubes are turned off by application of a gate voltage, and the metallic tubes are subjected to a large current $[60,77]$. The large current densities through the metallic tube cause the tubes to breakdown, perhaps by joule heating. The remaining CNTs are semiconducting.

Also selective electro chemical reactions $[78,79]$, where the resistivity of the metallic tubes is increased by several orders of magnitude, has been applied again resulting in the removal of the metallic CNTs, from the device.

Perhaps one of the most exciting methods for the selection between metallic and semiconducting tubes is dielectrophoresis, whereby the difference in the dielectric constants between metallic and semiconducting CNTs allows selective alignment [77, 80-84]. The metallic tubes have a much larger dielectric constant and as such preferentially align to the electric fields. The semiconducting CNTs will remain unaligned in the solution unless in the case of poor suspension, where they will be aligned along side the 
metallic tubes, due to van der waals interactions. Burn off can then be applied to devices where a mixture of metallic and semiconducting CNTs have aligned. Or where good suspensions have limited the bundling, the remaining dispersion solution should contain only semiconducting CNTs, the metallic CNTs having aligned across the electrodes on the substrate surface.

The field dependent device characteristics for back gated CNTs are now discussed more specifically.

\subsection{Carbon Nanotube Field Effect Transistors}

Inherent CNT FETs in air are all observed to behave as p-type devices. Originally it was thought that this was due to $\mathrm{O}_{2}$ from the atmosphere chemically doping the CNTs along the sidewalls. However, when CNTs were annealed in a vacuum or a gas such as nitrogen the CNT FETs became increasingly ambipolar and then n-type $[72,85,86]$. Re-exposure to atmosphere was seen to reverse the effect and the CNT FETs reverted back to p-type. These experiments have shown that the Schottky barrier nature of the contacts is influenced by the oxygen from the atmosphere affecting the CNT electrode interface.

Following from the discussion of the Schottky barrier CNT FETs above in Sections 2.2 locally applied gates have been successfully applied to produce p-type and n-type devices on the same chip, the crucial step towards logic devices. However, the choice of electrode metal was again seen to be an important consideration with similarly prepared devices with $\mathrm{Cr} / \mathrm{Au}$ electrodes remaining p-type after the anneal and Co electrodes producing n-type devices for one group [87].

\subsubsection{CNT Doping}

Carbon nanotubes can also be doped in the conventional, chemical doping sense, by the exposure to gas dopants such as bromide and iodine to provide p-type dopants and to potassium and cesium to provide n-type dopants [67,88-90]. Exposure to amine containing groups has been observed to provide enough dopants to create $n$-type devices $[16,91,92]$ as well as use of an amine plasma [14] and even proteins [93]. Ex- 
posure to some polyimine groups has been shown to be reversible $[15,94]$ with others providing molecules which can both accept and donate charge to the same nanotube depending upon external conditions [95].

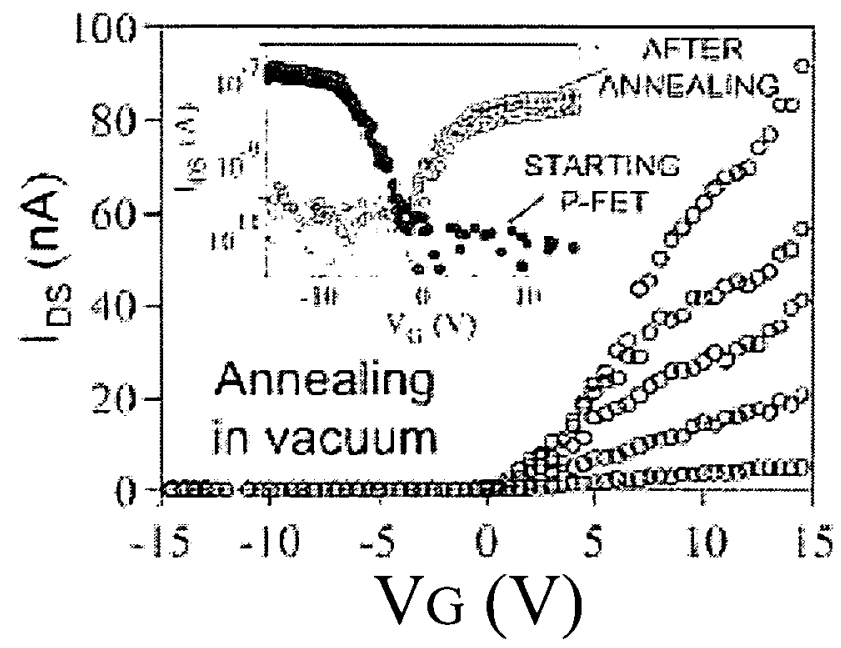

(a) A p-type CNT FET is annealed in a vacuum to become a n-type device. The inset shows the device before and after annealing

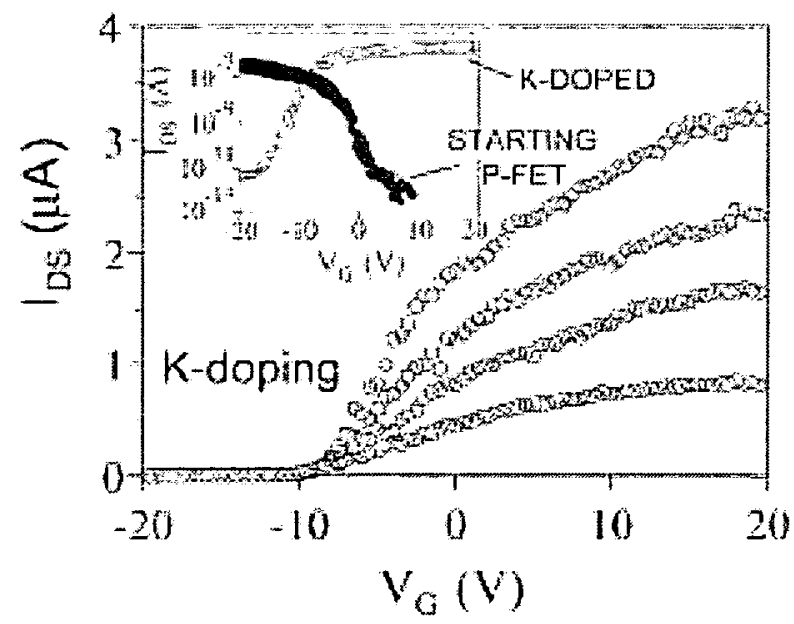

(b) A p-type CNT FET is doped with potassium to become a n-type device. The inset shows the devices before and after doping.

Figure 2.10: p-type CNT FETs are converted to n-type devices by both vacuum annealing and chemical doping taken from work by the IBM group [85]

The chemical doping of CNTs for FET devices has been shown to be different from the locally applied gate voltage effect primarily in the sense that the threshold voltage shifts for the chemically doped devices $[67,72,85,86]$. A comparison between the two 
different methods of doping was made at IBM and the resulting I-V curves are shown in figure 2.10 taken from work by Derycke et al. [85]. The threshold shift is a clear sign that the addition of electrons is responsible for the doping observed in these devices rather than an alteration at the CNT/electrode interface. In other work with a potassium doping and annealing stage [67] on a thin gate oxide, devices with highly improved n-type CNT FETs were achieved. The potassium provided the threshold voltage shift and the n-type dopants, whilst the annealing stage improved the bulk doping and the injection from the source electrodes.

\subsubsection{CNT Logic Devices}

The two distinct routes, gate induced doping and chemical doping give researchers the ability to carefully control the devices. With the recent success of n-type and ptype devices being produced carbon nanotube logic devices have been created such as NOT [72,75] NOR, OR, NAND and AND gates [75], ring oscillators, $220 \mathrm{~Hz}$ [75] $5 \mathrm{~Hz}$ [96] and CNT transistors have been seen to operate at $2.6 \mathrm{GHz}$ frequencies [28]. Carbon nanotubes were also successfully integrated with silicon NMOS devices by a direct growth method [97]. The ability of carbon nanotubes to be controlled by local gating has lead to both inter ( 2 separate tubes) and intra (one tube only) CNT devices [72] and also the ability to induce optical emission from hole and electron recombination in a device $[29,76]$. More recently local gating of a single carbon nanotube has created an ideal $p-n$ diode [98], thus confirming the local gating method to successfully control the CNT electronic behaviour without any damage to the structure of the CNTs.

\subsubsection{CNT Electronic Device Summary}

At present techniques for positional control of CNTs still need to be refined and a key requirement is the ability to determine the CNT electrical behaviour to order, rather than the unknown chiralities from each growth process that is the current state of the art. Nevertheless some promising characteristics have been shown, with good local control of the CNT gate allowing device behaviour to be controlled [86], improved dopant uniformity and injection through annealing [67] and the ability to use a single molecule for different dopant effect [91]. 
CNTs have been shown to have uses not just as interconnects and field effect transistors, but also as field emission devices, for applications such as television screens and as electron sources for e-beam lithography $[26,99-102]$. By growing the CNTs perpendicular to the substrate surface, high quality displays and electron sources can be made. In this work no attempt to explore the field emission properties of CNTs has been made so no thorough review of the field will be made here. However, it is interesting to note the stunning versatility of the CNT material.

It is with a view to the promising electronic device behaviour of carbon nanotubes that this investigation started, with emphasis on controlling the positioning of the CNTs for FET applications. 


\section{Chapter 3 Characterisation Techniques: Spectroscopy and Electronic Device Characterisation}

The approach of functionalising CNTs using chemical modification techniques was adopted here as a first step in the utilisation of carbon nanotubes (CNTs) for molecular electronics. In order to draw conclusion from any experiment carried out on the carbon nanotubes, sensitive spectroscopic techniques are required. Throughout this investigation the main techniques used have been Raman spectroscopy and X-ray photoelectron spectroscopy (XPS). Due to the inherently small size of the samples and the complexity of the arrangements all spectroscopic analysis of CNTs is non-trivial.

The advantages of both Raman spectroscopy and XPS along with the information which can be gathered is described in the following chapter in Sections 3.1 to 3.2. Some practical advice on accurate analysis of the experimental data will be given.

Microscopy is also a useful tool in the study of carbon nanotubes. Scanning electron microscopy (SEM), transmission electron microscopy (TEM) and atomic force microscopy (AFM) have all been applied during this work, however merely as an inspection tool so no background description will be made here.

The subsequent devices were then characterised by microelectronic probe station techniques. In doing so the electronic behaviour of the CNT devices could be analysed. In particular whether the device was field-dependent or not. Along with the expected behaviour CNT device probing will be discussed in Section 3.3.

\subsection{Raman Spectroscopy}

Raman spectroscopy is a powerful tool for understanding the molecular structure of molecules. A general understanding of the principles of Raman spectroscopy can begin 
from the polarizability of a molecule and the quantum theory of radiation. A clear discussion of the technique is given by Banwell [103]. A molecule in a static electric field will suffer some form of distortion, whereby + ve atoms in the nucleus will become attracted to the -ve pole of the field, and -ve atoms in shell will become attracted to the + ve pole of the field. Such a distortion causes an induced dipole moment, $\mu$, and the molecule is said to be polarised. The size of $\mu$ depends on the magnitude of the applied field $\vec{E}$ such that,

$$
\mu=\alpha \vec{E}
$$

where $\alpha$ is the polarizability. The polarizability will change periodically with time as the molecule vibrates or rotates, therefore it is more accurate to consider

$$
\mu=\alpha_{0}+\Delta \alpha \sin 2 \pi \nu_{v i b} t
$$

where, $\alpha_{0}$ is the average of the polarizability, $\Delta \alpha$ is the rate of change of polarizability with the vibration and $\nu_{v i b}$ is some kind of internal frequency. Now for a Raman setup, consider a stream of photons incident upon a molecule. The photons will strike the molecule with an energy $E=h \nu$, where $\nu$ is the frequency of the radiation and $h$ is Planck's constant. The electric field experienced by each molecule due to the incident radiation will also vary with time such that,

$$
\vec{E}=E_{0} \sin 2 \pi \nu t
$$

Therefore a description of the dipole moment is

$$
\mu=\alpha \vec{E}=\left(\alpha_{0}+\Delta \alpha \sin 2 \pi \nu_{v i b} t\right) E_{0} \sin 2 \pi \nu t
$$

expanded simply to give

$$
\mu=\alpha_{0} E_{0} \sin 2 \pi \nu t+\frac{1}{2} \Delta \alpha E_{0}\left\{\cos 2 \pi\left(\nu-\nu_{v i b}\right) t-\cos 2 \pi\left(\nu-\nu_{v i b}\right) t\right\}
$$


producing 3 output terms all with physical meaning. The first term showing the case when the scattered and incident frequency are the same, and the latter 2 terms due to the addition or loss of energy to the molecule, respectively [103, 104]. When a photon and molecule interact the molecular wavefunction is disturbed. For a very short time the system no longer exists in a stationary state, and exists in a virtual state. When the molecule returns to a stationary state it may or may not be different from the initial state, the possible outcomes are shown below in table 3.1 .

\begin{tabular}{|c|c|c|}
\hline Molecule energy change & Scattered radiation frequency & Type of scattering \\
\hline No energy change & $\nu$ & Rayleigh \\
gains energy & $\nu-\frac{\Delta \mathbf{E}}{h}$ & Stokes \\
loses energy & $\nu+\frac{\Delta \mathbf{E}}{h}$ & Anti-Stokes \\
\hline
\end{tabular}

Table 3.1: Raman scattering outcomes

The molecule can lose and gain energy only in accordance to quantal laws. Therefore any $\Delta E$ is the difference in energy between two allowed states, meaning a change in the vibrational or rotational energy of the molecule. In order for a molecule to be Raman active a molecular rotation or vibration must cause a change in a component of the molecular polarizability. The prediction of such changes are difficult and are best described by group theory analysis which will not be done here, complicated further by the fact that polarizability is a tensor component. For practical purposes though it is necessary to note that the radiation incident during Raman spectroscopy is usually in the visible or ultraviolet region, $(1 \mu \mathrm{m}-10 \mathrm{~nm}$ ) and therefore much greater than the size of a molecular bond which is about $0.1 \mathrm{~nm}$. As such radiation can only sense the average polarizability in various directions [103].

The intensity of the stokes and anti-stokes signals will be relatively weak in comparison to the Rayleigh scattering line. The anti-stokes will be especially weak as it is statistically less likely to occur. The giving of energy from the molecule to the scattered radiation that an anti-stokes transition dictates, implies that the molecule was originally in an excited state, an occurrence which is known to be less likely due to Boltzmann's distribution and the rules of statistical mechanics. Sensitive equipment is therefore re- 
quired to detect these scattered photons. However, it is useful to note that symmetric vibrations result in intense Raman lines, whilst non-symmetric vibrations can often be weak, or even not visible. In the case of carbon nanotubes the key Raman active modes are highly visible.

\subsubsection{Raman Spectroscopy of Carbon Nanotubes}

A widely accepted spectroscopic technique for the study of carbon nanotubes is Raman Spectroscopy. A great amount of pioneering research has been done with Raman spectroscopy, largely by the group led by M. S. Dresselhaus at M.I.T. [32, 105-110], making it one of the more reliable experimental techniques at present in nanotube characterisation. It has been possible to perform single-tube Raman spectroscopy [32] and novel techniques have given insight into the photon interactions of the CNTs and provided much information regarding their characteristic structure. Single nanotube resonant Raman spectroscopy can be applied to accurately determine the $(n, m)^{1}$ indices of the tube, due to the strong coupling between the incident or scattered photons and the electronic transitions between the conduction and valence bands of the tubes $[32,105,106]$. To do so the radial breathing mode is used as a first point of characterisation, which is then correlated with the G-band vibrations of the CNTs. Figure 3.1 shows the RBM and the G-band vibrations on the CNT structure.

The Raman spectra of a CNT can immediately determine whether or not the tube is structurally intact, which is why it is often the first point of characterisation. The RBM is particularly sensitive to the $(n, m)$ of the individual CNT making it a useful first point of identification for the tube structure. From the frequency of the RBM the nanotube diameter $d_{t}$ can be simply determined from equation 3.6 .

$$
\omega_{R B M}=\frac{\alpha}{d_{t}}
$$

where $\alpha=248 \pm 2 \mathrm{~cm}^{-1} \mathrm{~nm}$ according to experimental verification $[32,105,106]$. Tunable lasers could be powerfully applied to determine the $(n, m)$ from the RBM alone, however, tunable lasers are not readily available and the G-bands have to be considered to double check that no large errors were made in the initial determination stages.

\footnotetext{
${ }^{1}$ See Section 2.1 for a description of the CNT structure.
} 


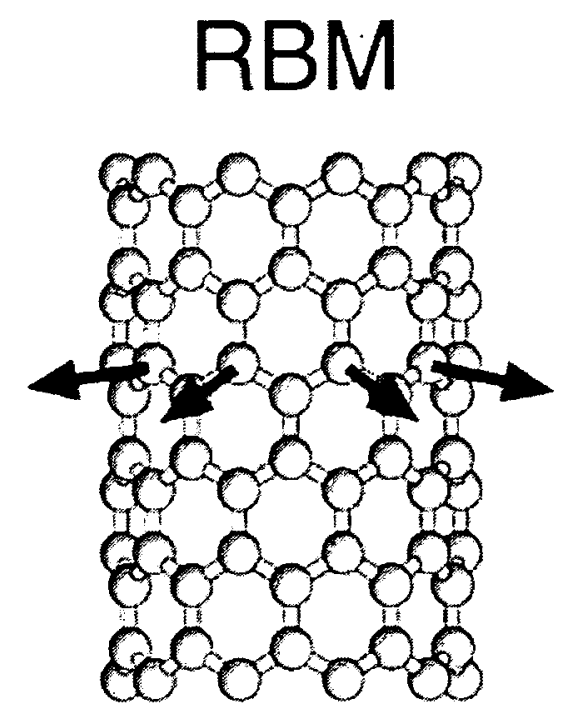

(a) The radial breathing mode (RBM): All carbon atoms are moving in phase in the radial direction.

\section{G-band}

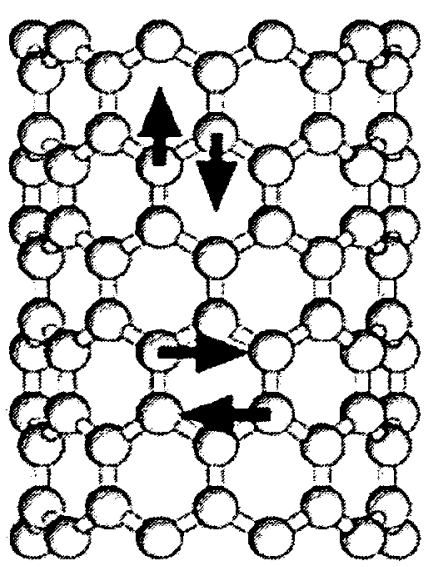

(b) The carbon nanotube G-band: neighbouring atoms are moving in opposite directions along the surface of the tube as in $2 \mathrm{D}$ graphite.

Figure 3.1: The vibrations of the radial breathing mode (RBM) and the G-band. Taken from [108]

The G-band is also sensitive to the incident laser energy and can be split into two parts, $\omega_{G}^{+}=$circumferential and $\omega_{G}^{-}=$along the tube axis, as shown in figure 3.1(b). However, this is only useful in the case for single nanotubes. In the case of bundles or ropes of nanotubes as observed here, the same modes are visible but arising from many different tubes in a single bundle.

The intensity of the tube vibration will depend on whether that tube was in resonance with the laser energy or not. The $633 \mathrm{~nm}$ wavelength as used here is particularly useful for researchers looking at bundles with both semiconducting and metallic tubes [111] as was the case here. Although the bundles of tubes and the resonance considerations make it difficult to accurately determine the structure of the tubes in any given bundle, it is still a powerful and useful technique. The presence of nanotube-nanotube interactions may cause changes in the vibrational frequencies of the SWNTs in the bundles, however these interactions are not important for the G-band [107] which is a useful determination of the SWNT structural integrity. The Raman spectra of a bundle of tubes can also be readily applied to determine the diameter distribution of the CNTs in the bundles $[32,89]$. 


\subsubsection{Raman Spectroscopy: Experimental Considerations}

In a standard Raman setup, light from a laser is directed towards the sample of interest. Usually most of the light emitted from the sample has the same frequency as the incident light (Rayleigh scattering), however, if energy is exchanged between a photon and a molecule during a collision, the emitted radiation will be either higher or lower in frequency. The theory and experimental considerations of Raman spectroscopy are described in the textbook by Banwell [103] and were discussed briefly in the introduction to this chapter. The experimental setup is described below in figure 3.2.

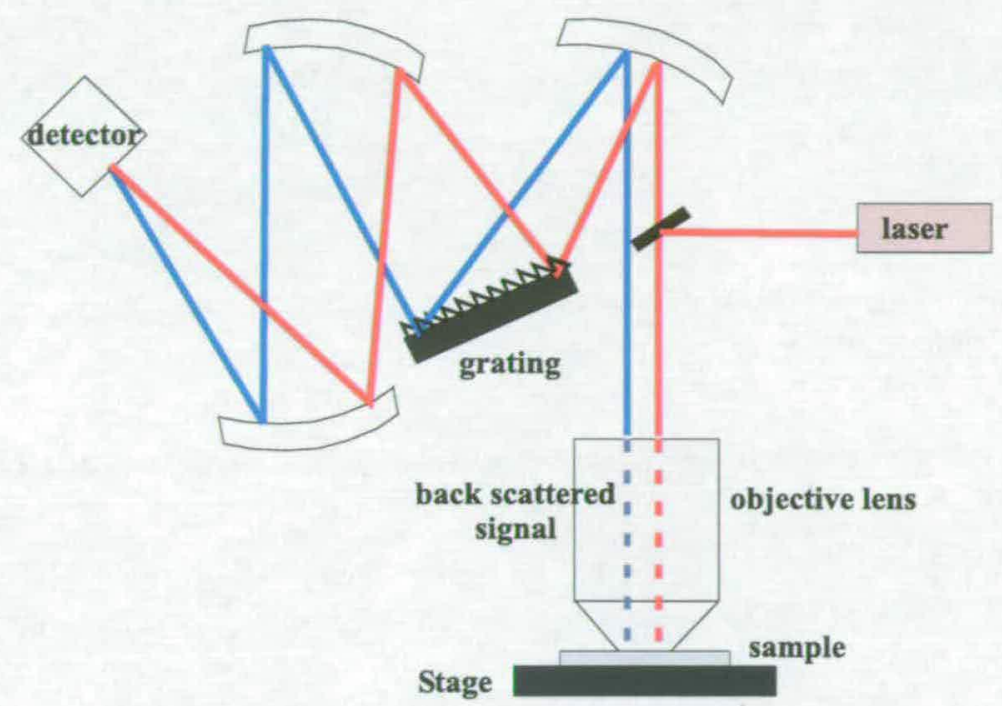

Figure 3.2: An example of the Raman setup used.

Here the LabRam 300 with $633 \mathrm{~nm}$ laser line at $100 \mathrm{~W}$ power was used. The incident laser is directed towards the source, whereby an optical light source has been used previously to focus the image. The reflected light is then collected and sent to a detector. The set-up shown here is for backscattering geometry which was used throughout the investigations.

\subsubsection{The Carbon Nanotube Raman Spectra}

For a change to be seen in the Raman spectra a molecular rotation or vibration has to change a component of the molecular polarizability. A typical spectra for a carbon nanotube sample is shown in figure 3.3. 


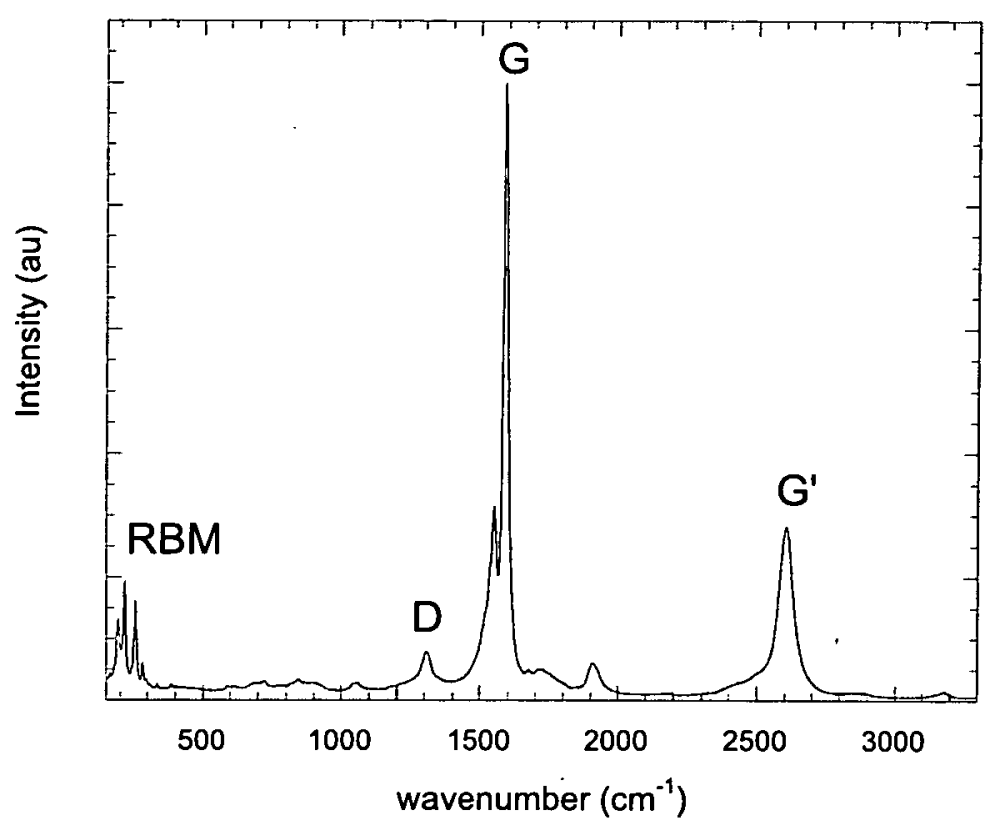

Figure 3.3: An example of the typical Raman spectra of CNTs.

The low frequency resonance which is observed at approximately $200 \mathrm{~cm}^{-1}$ is known as the radial breathing mode of the carbon nanotube and relates sensitively to the diameter of the tube. The higher frequency peaks at $1600 \mathrm{~cm}^{-1}$ and $1300 \mathrm{~cm}^{-1}$ are known as the G-band and the D-band respectively. The G-band refers to $\mathrm{sp}^{2}$ bonded carbon (e.g. CNTs or graphite) and was discussed above whilst the D-band can be used as a gauge of the quality of the CNTs. The higher the intensity of the D-band implies a higher level of defects on the tube samples $[32,105,106,108,112]$.

The D-band is also accompanied by its second harmonic the G'-band at approximately $2600 \mathrm{~cm}^{-1}[32,105,106,108,112]$. The D-band and the $G^{\prime}$-band both arise from defects on the CNT structure, or due to attached molecules by functionalisation, and are dispersive i.e. they change in frequency when the laser excitation energy changes. However, the mechanism is slightly different with the D-band arising due to a photon interacting with a defect, and the G'-band with 2 phonons at specific wavevectors $q$ and -q. See $[32,105,106,108,112]$ for a full discussion.

These higher frequency peaks, G-band and D-band, depend less on the CNT diameter and tend not to vary too much between different $(n, m)$ tubes. However the G-band is presumed to shift upon high levels of covalent functionalisation $[108,111]$ where electron acceptors (p-type dopants) should shift $\omega_{R B M}$ and $\omega_{G}$ to higher frequencies 
and electron donators (n-type dopants) should shift $\omega_{R B M}$ and $\omega_{G}$ to lower frequencies.

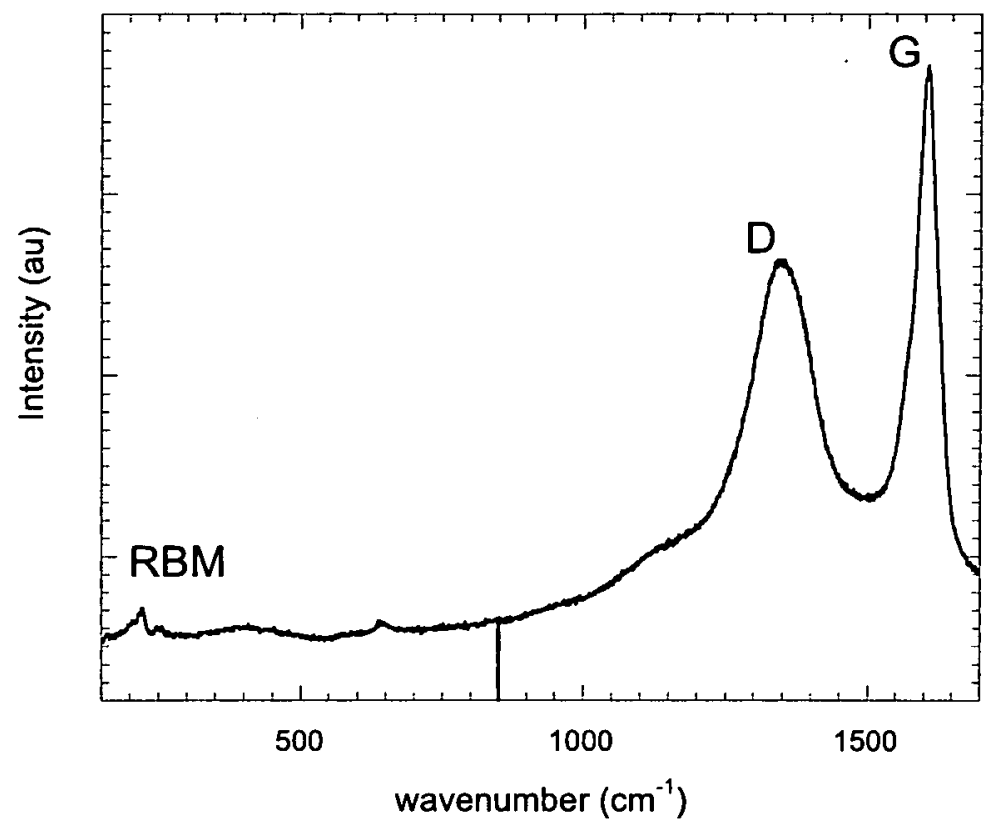

Figure 3.4: An example of the Raman spectra of CNTs functionalised using an aggressive acid.

It is therefore essential after various stages of functionalisation to check the structural integrity of the tubes and in practice Raman Spectroscopy has been shown to be a quick and reliable method of doing so. For example, an acid oxidised sample of CNTs as observed in figure 3.4 has clearly increased defect content and the RBM is no longer clearly visible. The loss of the RBM may be due to a broadening of the band or an extreme down shifting $[108,111]$. It can therefore be concluded that the acid functionalisation process used was detrimental to the nanotube structure. It may also be an indication of carboxylic acid groups being attached to the sidewalls of the CNTs. Such attachments would alter the $\mathrm{sp}^{2}$ carbon bonding of the sidewall atoms to a more disordered arrangement of $\mathrm{sp}^{3}$ hybridisation, clearly observed by a dramatic increase in the D-band intensity. At this stage it is difficult with Raman alone to determine how much of the D-band is caused by defects or by functional groups bonded to the sidewalls. Further investigation means, such as XPS are required ${ }^{2}$.

\footnotetext{
${ }^{2}$ TEM or FT-IR are also useful techniques for CNT functionalisation characterisation. However, these are not discussed here.
} 


\subsubsection{Raman Summary}

Raman spectroscopy is a quick and non-intrusive way of deducing whether the CNT structure is intact, and to gain an indication of the average tube diameter in a bundle. As the theoretical understanding of the origins of the RBM, G-band and D-band are now well established, it is possible to deduce the $(n, m)$ values for a given CNT when single nanotube Raman spectroscopy is employed. For the purposes here, where bundles of CNTs are characterised, the $633 \mathrm{~nm}$ laser line is most useful as many CNTs, both metallic and semiconducting, can be probed at this wavelength. The average properties of the bundles, such as CNT diameter distribution and the structural integrity of the CNT can be easily determined.

\subsection{X-ray Photoelectron Spectroscopy (XPS)}

$\mathrm{X}$-ray photoelectron spectroscopy is a powerful technique, whereby the core electrons of an atom of a molecule are probed by a X-ray source. The experiment is set up using a monochromatic X-ray source whereby the X-rays have energy capable of probing the innermost core of the atom or molecules. The method behind XPS comes from the photoelectron effect. Consider the diagram shown in figure 3.5, taken from [103].

When the energy of the incident photon is higher than the binding energy of the electron, the electron will be emitted. Similarly, when an X-ray collides with an atom or molecule an excitation takes place and an X-ray is emitted with a different velocity. Unlike the Raman excitations the energy is not quantised. All that is required is that the incident energy is larger than the binding energy. The relationship between the incident X-ray energy, the emitted electron energy and the binding energy of the sample is described by equation 3.7 [103, 113-115].

$$
h \nu=E_{K E}+B E
$$

where $h$ is Planck's constant and $\nu$ is the frequency of the incident X-ray form the source, $E_{K E}$ is the kinetic energy of the emitted electrons and $B E$ is the binding energy of the atom or molecule. For the apparatus used here, the VG Scientific Sigma Probe, the incident radiation is from an $\mathrm{Al} k_{\alpha}$ source for which $h \nu=1486.6 \mathrm{eV}$. 


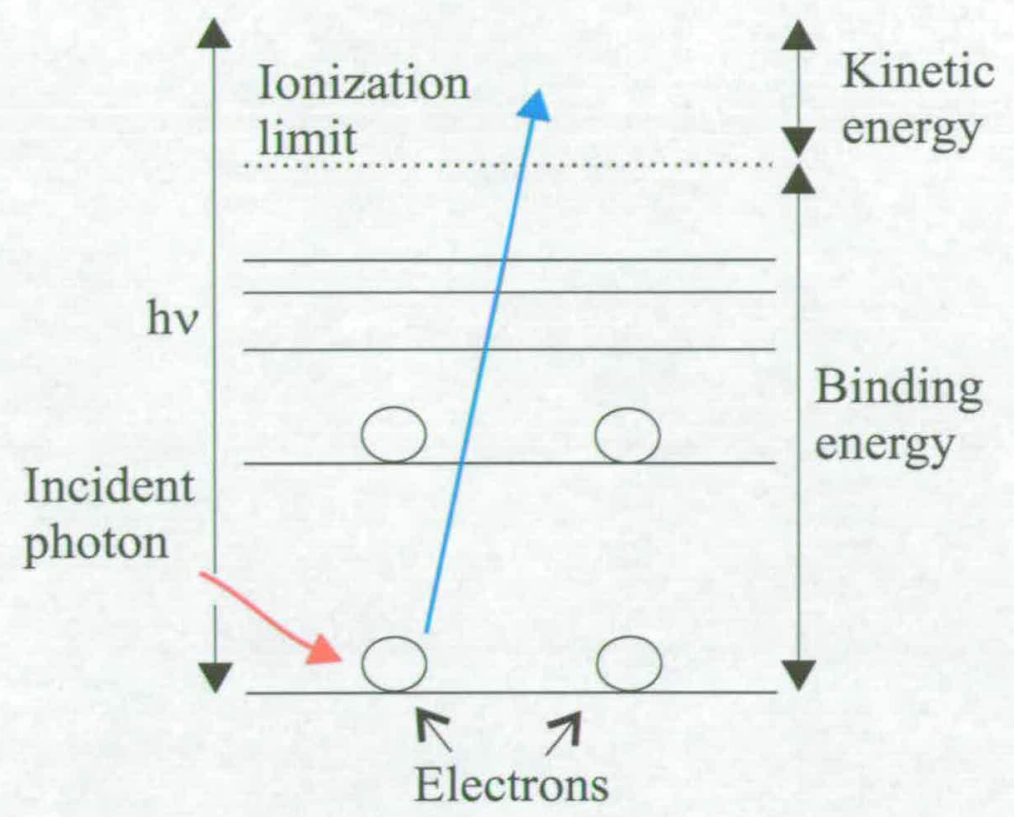

Figure 3.5: An example of the photoelectron effect. When the energy of the incident photon is higher than the binding energy of the electron, the electron will be emitted.

Equation 3.7 can be simply rearranged to give the binding energy of the electron,

$$
B E=h \nu-E_{K E}
$$

The energy of the emitted electron will depend sensitively on the bond being formed within the atom or molecule. Although chemical bonds do not alter the energies of the core electrons as dramatically as for the valence electrons, there is still a clearly observable energy shift, due to the electron shielding effects. Ultra high vacuum is required for the successful probing of the core electrons and valence electrons ${ }^{3}$, as the electrons are highly reactive and as such limited reaction with other matter is essential.

The velocity of the electrons emitted depends sensitively on the level of the core electron. For example electrons can be emitted from the $1 s, 2 s$, or $2 p$ level of fluorine. The fluorine peak with the highest intensity is usually the F1s peak and as such it is usually used for analysis. The 1s peak is a single peak, and can be deconvoluted to determine physical information of the bond being studied. The $2 \mathrm{p}$ peak of a molecule will be more

\footnotetext{
${ }^{3}$ The technique for the study of valence electrons also follows the photoelectron affect as discussed here and is known as ultra-violet photoelectron spectroscopy.
} 
complicated due to the spin orbit splitting of the $2 \mathrm{p}$ level. A $2 \mathrm{p}_{\frac{3}{2}}$ and $2 \mathrm{p}_{\frac{1}{2}}$ both exist, with the latter being twice as likely and therefore having twice the intensity (e.g. an intensity ratio of 2:1), due to quantum mechanical considerations of the $s$ and $l$ quantum states [104]. For the $1 \mathrm{~s}$ shell where $l=0$ spin orbit coupling is not evident.

The binding energies of particular atoms or molecules have been extensively studied and can be found in many databases. Here, the NIST database [116] and the handbook of X-ray photoelectron spectroscopy [114] were the primary sources of reference. It is important to note here that the known binding energies of atoms and molecules are well defined and can be used as a fingerprint identification system. However it is up to the scientist to determine any factors influencing an individual experiment such as gun energy drift, contamination, possible cross linking etc. in order to understand the experimental results.

\subsubsection{Deconvolution of the XPS Spectra}

By use of known standard peaks for the XPS of atoms it is possible to deduce particular information regarding the bonding taking place. For example, the ionic and covalent regions of the $\mathrm{F} 1 \mathrm{~s}$ peak of fluorine, lie in different regions, $686-687.5 \mathrm{eV}$ respectively $[9$, $114,117-119]$. When a spectra of fluorine is obtained it will consist of a combination of peaks within the F1s range. In reality it is possible that a spectra will contain both semi-ionic and covalent F1s components. It is therefore necessary to deconvolute the spectra to calculate the relative intensities of each bonding type.

To do this, specific software such as XPS Peak ${ }^{4}$ is required. It is necessary to consider the type of core electron being probed. In the case of carbon, oxygen and fluorine, studied here, the core electrons are in the s orbital shell. Therefore closest to the core of the molecule. The spectra will be deconvoluted using a combination of Gaussian peaks in most cases here, such as the $01 s[90,113,114,121], F 1 s[10,114,117,119]$ and the C1s $[9,90,114,119]$ peaks. However, other types of peaks exist, for example the sulphur S2p, which requires a 70:30 Lorentzian:Gaussian peak is often used in the literature [122-126].

In principle, the shape of the peak does little to alter the relative intensity so long as the

\footnotetext{
${ }^{4}$ XPS Peak is freely available software on the web [120], written by Raymond Kwok.
} 
choice is consistent; most authors use a Gaussian peak. The peaks are then fitted within the software using best fit algorithm consistent with the choice of peak shape and any other restrictions made by the user. A fit is considered when the value of $\chi^{2} \leq 1$ and in practice the fit is repeated until no further improvement of $\chi^{2}$ can be made.

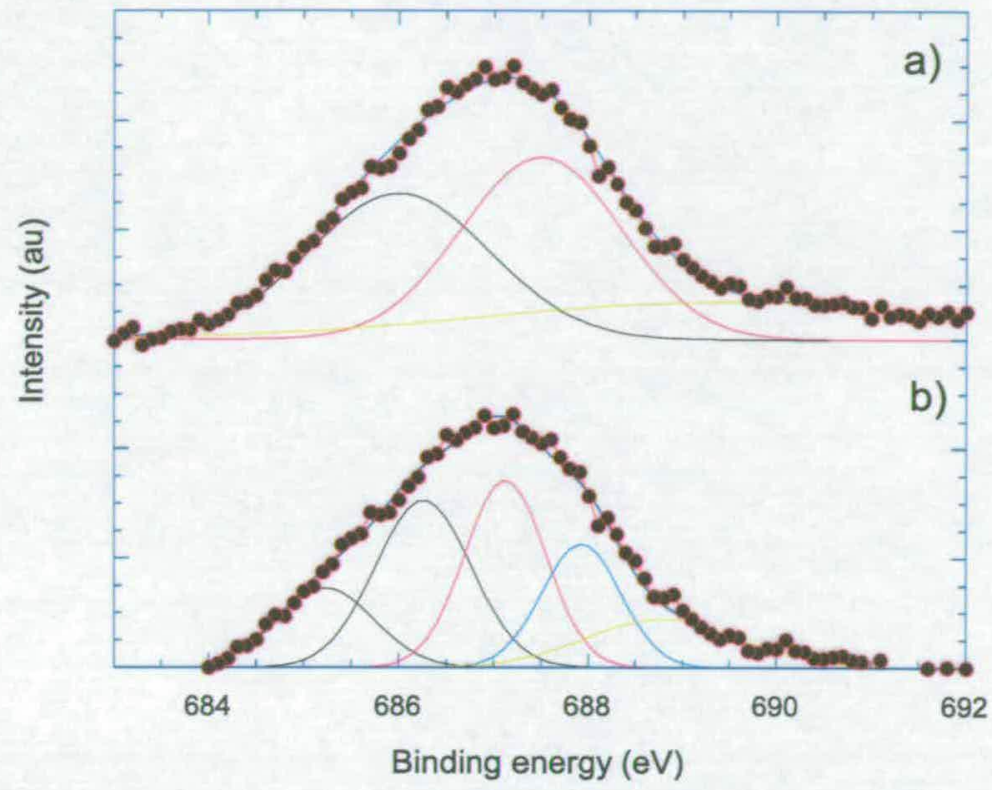

Figure 3.6: An example of the F1S peak, fitted to a) Semi-ionic and covalent fluorine plus background effects, and b) multiple Gaussian peaks at $1 \mathrm{eV}$ spacing.

It is important to note that any peak can be made up of an infinite number of Gaussian peaks. Therefore it is usual to fit with the minimum amount of peaks possible in the first instance. An example F1s peak is shown in figure 3.6, the peak has firstly been fitted using the minimum amount of peaks possible, 3 peaks. The second shows multiple peaks at $1 \mathrm{eV}$ spacing, being fitted to the same curve. Both situations make a good match between the fitted and the measured data. Clearly before deconvoluting it is necessary to have an indication of what may or may not be present on the sample, and fitting to the minimum peaks required is always advised.

There may however be situations where deconvoluting of the XPS peak is not possible without further spectroscopic data. For example in the $\mathrm{C} 1 \mathrm{~s}$ peak of fluorinated carbon nanotubes, the binding energy of carbon to fluorine and carbon to oxygen overlaps $[9,90,114,117,119]$. As the samples have been functionalised with fluorine and atmospheric contamination of oxygen is expected, it is difficult to know how to deconvolute the spectra. In such a case, the $\mathrm{C} 1 \mathrm{~s}$ peak is an unreliable means of characterising 
the functionalisation, and the O1s and F1s peaks must be studied alone.

\subsubsection{Experimental and Analysis Considerations}

In order to make efficient use of the data acquired from the XPS experiments it is essential that the sample preparation is consistent when comparisons are to be made. It is also often necessary to shift the binding energies measured during the experiment using a well defined element. For example, shifting the maximum of the C1s peak to lie at a binding energy of $284.6 \mathrm{eV}$, which corresponds to $\mathrm{sp}^{2}$ bonded carbon. The $\mathrm{C} 1 \mathrm{~s}$ peak can only be used if it is known that the tube structure has remained intact and some form of confirmation technique such as Raman Spectroscopy discussed in Section 3.1 has been performed. In the literature metals such as gold are often used for this purpose when C1s peaks would not be suitable.

The XPS data can also be applied to calculate the atomic percentages of each element present on the sample. In these experiments the ratio of carbon to fluorine, oxygen, nitrogen and sulphur has provided valuable insight into the functionalisation processes carried out. The atomic percentages have been calculated within the software present from VG Scientific with a linear background used in the case of all elements studied here. The atomic percentages are then calculated using Al Wagner coefficients [113], which account for the different atomic weights and sensitivity of the particular element to XPS characterisation. In doing so, XPS has shown to be a valuable tool for the accurate and expedient determination of the CNT functionalisation. Together with the binding energies, atomic percentage data can be used to determine the success and failures of any given functionalisation process. Further spectroscopic techniques such as Fourier transform infrared spectroscopy (FT-IR), would also be valuable tools for CNT functionalisation characterisation. However, limited access to such equipment was available.

\subsubsection{XPS Summary}

X-ray photoelectron spectroscopy (XPS) is a powerful tool, where the incident high energy X-ray can probe the bonding via the binding energy of the core electrons. The technique can be used to accurately determine the presence of elements on a surface, 
the spectra for each element can then be deconvoluted to provide precise information on the bonding behaviour. For practical use several considerations are needed, such as the possibility of energy overlap between several different types of molecules, and a consideration of the peak shape used for fitting techniques.

\subsection{Electrical Characterisation}

After functionalisation of the CNTs, the CNTs were used as the channel for carbon nanotube field effect transistors (CNT FETs). To characterise the behaviour of the CNT devices electronic probe techniques were carried out. The CNT FETs were in turn compared with standard metal oxide semiconductor field effect transistors, MOSFETs, to characterise their behaviour. A brief description of the MOSFET in comparison to the CNT FET is given before the probing method is described.

\subsubsection{Field Effect Transistors}

Field effect transistors (FETs) have revolutionised modern information technology. Due to advancements in semiconductor microfabrication techniques, millions of transistors can be created on a single chip leading to fast output speeds and high throughput of data handling. In particular through the development of complementary metal oxide semiconductor (CMOS) devices, where n-type and p-type FETs are made in parallel to provide logic applications. The constant demands for faster and cheaper computers have meant that electronic engineers have had to miniaturise the standard CMOS devices according to the semiconductor technology road map, following Moore's Law [1].

Shown in figure 3.7 are schematic representations of a MOSFET and a CNT FET. In the MOSFET structure regions of highly n doped silicon, embedded in a p-type silicon wafer, are ohmically contacted to a source and drain electrode. The semiconductor regions are isolated from the gate electrode by a region of high quality thermally grown $\mathrm{SiO}_{2}$, as shown in the diagram. When a large positive voltage is applied via the gate electrode, the mobile charge carriers, for this diagram electrons, form an inversion layer known as the channel. Electrons can then flow freely between the source and drain electrode via the conductive channel. 


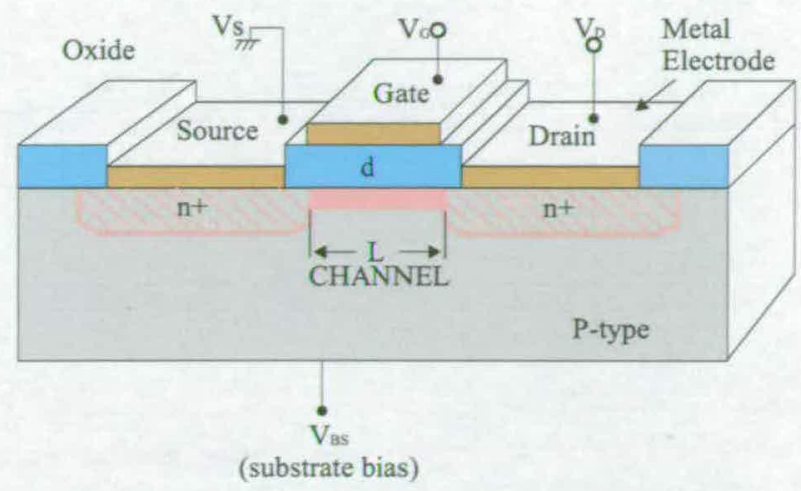

(a) The standard n-type FET schematic, taken from [127].

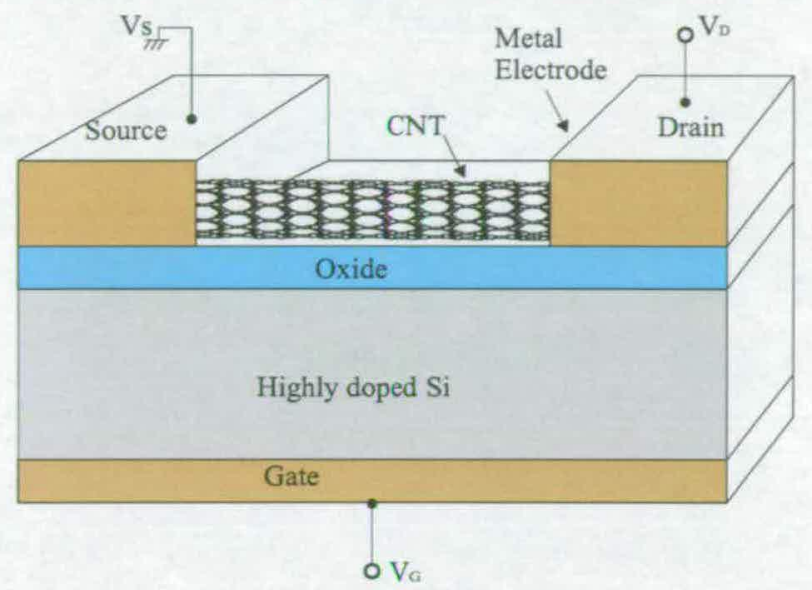

(b) A schematic of a CNT FET.

Figure 3.7: Schematic of a standard FET and a CNT FET for comparison

Figure 3.8 shows the characteristic outputs for both n-type and p-type MOSFETs in enhancement mode. Under the application of a positive bias, the n-type MOSFET begins to turn on. Looking to the schematic of the device structure 3.7(a) this effect can be explained by the field created by the gate electrode. When a large positive bias is applied at the gate the electrons in the bulk silicon are attracted towards the region, whilst the p-type carriers in the bulk silicon are repelled. When the gate field is sufficiently large the mobile electrons then form a channel bridging the two $n^{+}$doping wells and conduction takes place. The reverse is true for the p-type MOSFET, whereby application of a large negative gate field attracts the positive p-type carriers and p-type conduction channel is formed [127-129]. The performance of the MOSFET depends on several factors, in particular the doping level, for the amount of charge carriers present and the field required to provide an inversion layer. The gate oxide thickness is also important to isolate the gate electrode but not be so thick that the required field is too high. 


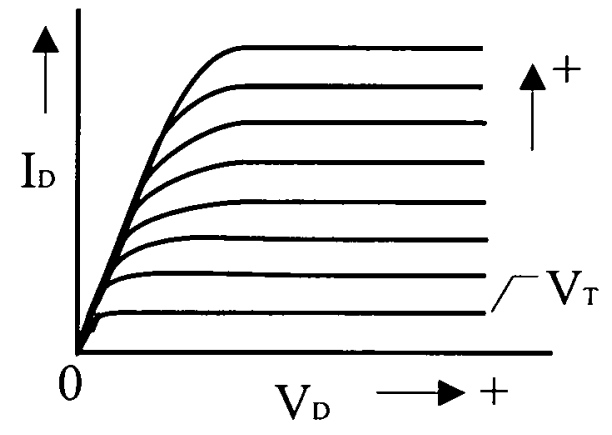

(a) For enhancement mode n-type FET drain current versus drain voltage, taken from [127]. The device is normally off.

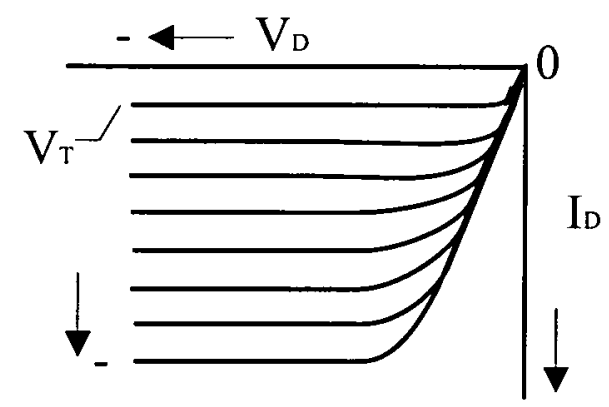

(c) For enhancement mode p-type FET drain current versus drain voltage, taken from [127]. The device is normally off.

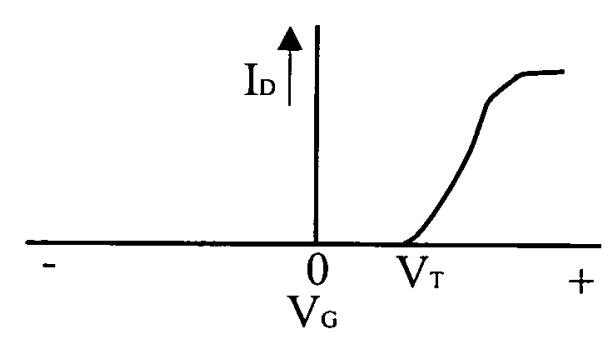

(b) For enhancement mode n-type FET drain current versus gate voltage, taken from [127].

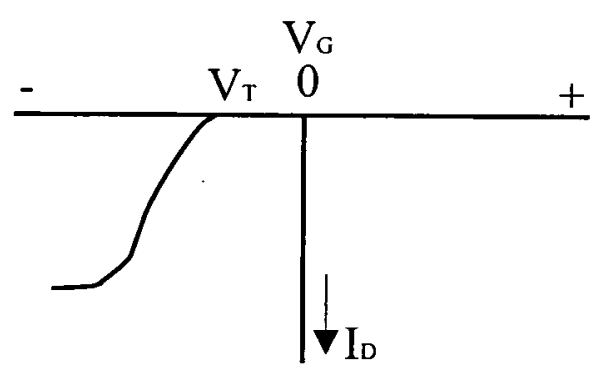

(d) For enhancement mode p-type FET drain current versus gate voltage, taken from [127].

Figure 3.8: The output for both a p-type and n-type FET in enhancement mode. The devices are normally off.

The operation of carbon nanotube transistors CNT FETs, is slightly different and the detailed operation mechanism of CNT FETs is still an active area of research. As discussed in Section 2.2 carbon nanotube and electrode contacts are often found to be Schottky barriers and not Ohmic contacts. Also from the diagram in figure 3.7(b), the CNT FETs do not have an inversion layer. The channel in this case is the CNT itself, however, there is no region to provide the extra mobile carriers, (unless chemical doping is performed). The conduction is therefore dependent upon the intrinsic CNT properties and the transport over the Schottky barrier. Intrinsic CNT FETs have all been observed to be p-type FET devices $[27,85,130]$. The Schottky barrier interface is now discussed below. 


\subsubsection{Metal-Semiconductor Interfaces}

A useful guide to understanding the process of conduction and the energy concepts is the band diagram of the metal-semiconductor contact, and example is shown in figure 3.9. The figures show the band diagrams for a n-type semiconductor. The arguments and solutions are however similar for p-type semiconductors. A Schottky diode is formed by bringing a semiconductor and a metal surface into intimate contact. At the interface between the semiconductor and the metal, a barrier will be formed and at thermal equilibrium the Fermi levels of the two materials must meet.

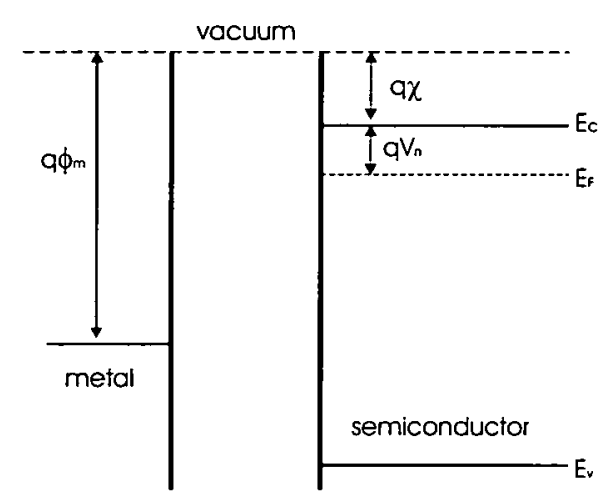

(a) The band diagram of the metal and the semiconductor before contact.

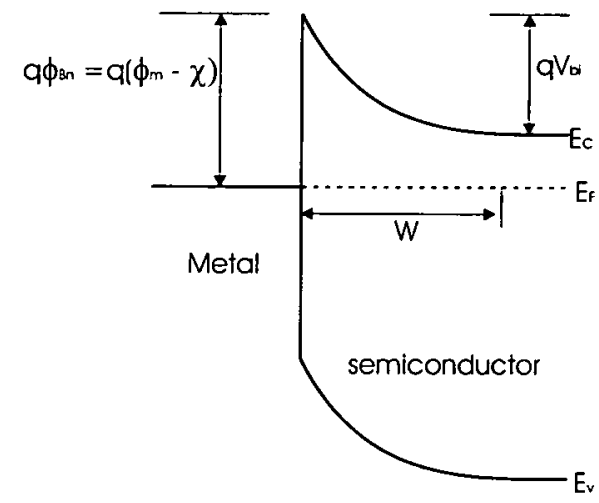

(b) The band diagram of the metal and the semiconductor after contact

Figure 3.9: For a n-type semiconductor making contact with a metal. taken from [128]

The transport of electrons across such a barrier has been a topic of intense scientific research for many years. By the middle of last century, the theory of current transport had made huge advances, and the theories produced then, still hold today. However, it is still the case that the current transport mechanisms are not easy to solve, and the precise method of conduction may at times be a complicated combination of one or more transport method.

An in depth discussion of the mechanisms involved in transport across the barrier can be read in the textbooks by Sze [127], Rhoderick [131] and Heinisch [132, 133], which the interested reader is advised to consult. The information in all of these texts has been taken from many papers written by the leaders in this field from the early $1900 \mathrm{~s}$ when the first point contact rectifiers were being used.

The four possible processes of conduction are listed below and depicted in figure 3.10 
[131].

1. The electrons have sufficient energy to pass over the potential barrier.

2. The electrons quantum tunnel through the potential barrier.

3. The electrons recombine with the holes in the space-charge region.

4. The holes are injected from the metal into the semiconductor.

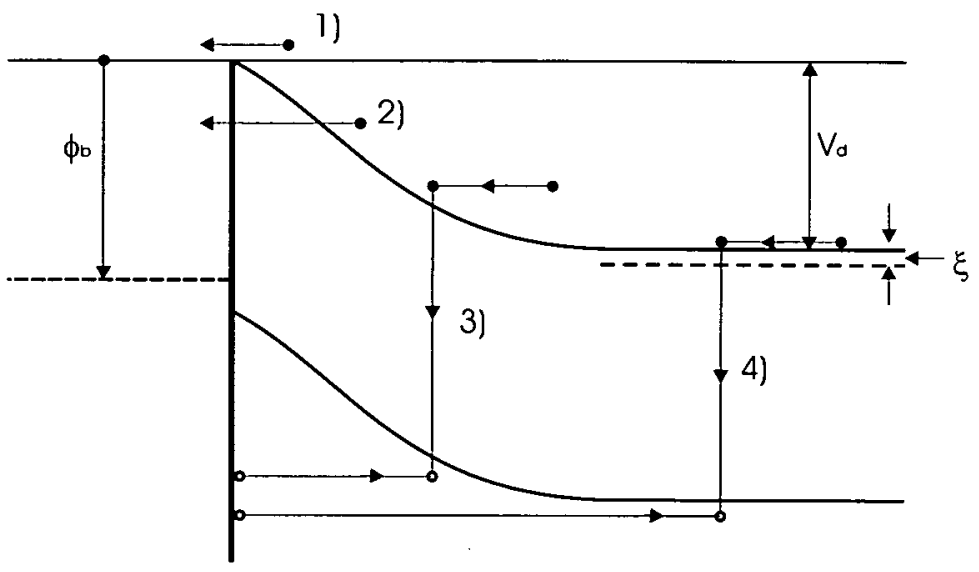

Figure 3.10: Current transport mechanisms.

In the case of a Schottky diode, the current transport is dominated by the motions of the majority carriers, in the n-type case, electrons and the p-type case, holes. When a field is applied to the Schottky diode, changes take place within the semiconductor. The electrons from either the semiconductor or the metal can transfer over the barrier, the direction of this current transport being dependent upon the applied bias.

\subsubsection{CNT Field Effect Transistors}

In the case of the CNT FETs, where the interface between the CNT and metal is a Schottky barrier, the band bending effects are the dominant factor for electrical conduction. Shown in figure 3.11 is a description of the band bending of CNT devices. Figure 3.11 is taken from work by the IBM group published by Derycke el al. [86] and is used to illustrate the difference between n-type behaviour due to the CNT/electrode contacts, (a) and (b) in comparison to n-type behaviour due to CNT doping (c) and (d). 

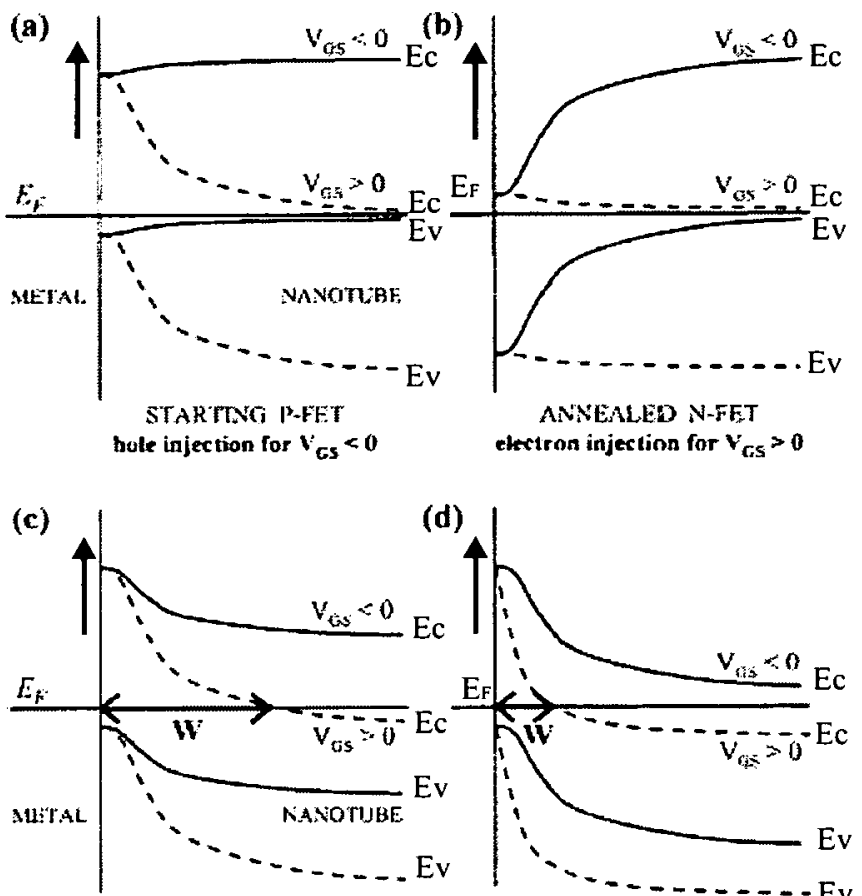

ANNEMLED S-FET

eketon injection for $\mathrm{V}_{\mathrm{CS}}>0$

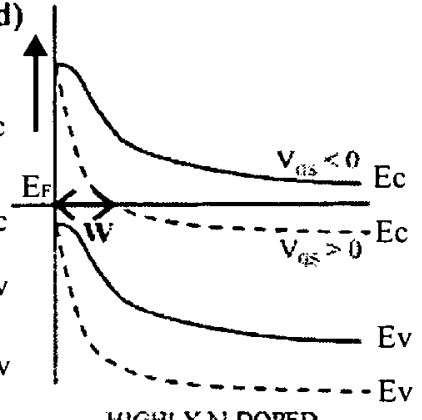

no current at any $r_{c s}$

HOHLY N-DOPED

electron tunneling through the barriar

Figure 3.11: The band diagrams of the CNT FET taken from [86] at $V_{D S}=0$. (a) bands of the starting p-type device in air. (b) the device after annealing in vacuum. (c) a nanotube FET that is not out-gassed but is n-doped by potassium, (d) as (c) but higher dopant level. $W$ denotes the barrier thickness for electron injection.

Firstly consider the p-type device in air, figure 3.11 (a), when $V_{G S}<0$ the barrier is too large for electrons from the conduction band to contribute to conduction in the device. Holes however, can conduct from the valence band through the barrier. When $V_{G S}>0$ neither holes from the valence band nor electrons from the conduction band can travel over the barrier and as such the device is turned off. Therefore for the conditions illustrated in figure 3.11(a), a current is achieved at $-\mathrm{V}_{G S}$ and not at $+\mathrm{V}_{G S}$, indicative of p-type behaviour. When the contacts have been annealed, figure 3.11(b), the energy levels between the metal and the CNT are altered. Conversely, to the case described in figure 3.11(a), at $V_{G S}>0$ electrons can travel from the conduction band. At $V_{G S}<0$ neither holes nor electroncs are capable of passing the barrier. The device is therefore behaving as a n-type FET.

Figures 3.11(c) and (d) show the effect of chemical doping. In figure 3.11(c), the CNT 
is weakly doped. At $V_{G S}<0$ and $V_{G S}>0$ there is no current flow observed for this device. The depletion width, at the CNT metal interface, is too large for electron conduction at positive $V_{G S}$ and due to the doping, the carrier density of the holes has been significantly reduced. The depletion width is wide and a large negative bias would be required to move the high concentration of charges, therfore no hole conduction takes place. The barrier is too high for the holes at $V_{G S}<0$ and as such there is an observed reduction in the overall $I_{d}$. When the CNT doping level is increased, figure 3.11(d), there is a reduction in the depletion region allowing electron conduction at positive $V_{G S}$. The depletion of hole carriers has been increased, therefore hole conduction at negative $V_{G S}$ is again not possible. The device here would act as a highly n-doped FET.

The exact conduction mechanism of CNTs and the interface between the CNT and the contact electrode is still not fully understood [53] and often the litertature on the subject can offer contradictory explanations. However, considering the mechanism of the CNT conduction described above, which is maintained along with experimental evidence $[85,86]$, and the difference between annealing contacts and chemical doping, the analysis of the current voltage characteristics for CNT devices produced are now discussed.

\subsubsection{Current-Voltage Characteristics}

To test the quality of the Schottky diode or an Ohmic contact, the first point of testing is usually the analysis of the current voltage (I-V) characteristics. The current can be measured as a function of voltage by making use of the HP 4165B probe table and ICCAP software. The I-V curve plotted is used to give an indication of the behaviour of the device, in particular whether or not it is field-dependent. The probe station applies a voltage to the relevant electrode, here the source electrode and the back gate, and measures the resulting current via the drain probe. Figure 3.12 shows the schematic representation of the $\mathrm{CNT}$ FET probe setup. The source, drain and gate probes are all clearly indicated.

By applying the source voltage and gate voltage the measured drain current gives an indication of the device type allowing for the CNT device to be characterised. The source and drain probes are needles whilst the gate probe is the chuck holding the CNT device. It is important that good contact is made between the electrodes and the 


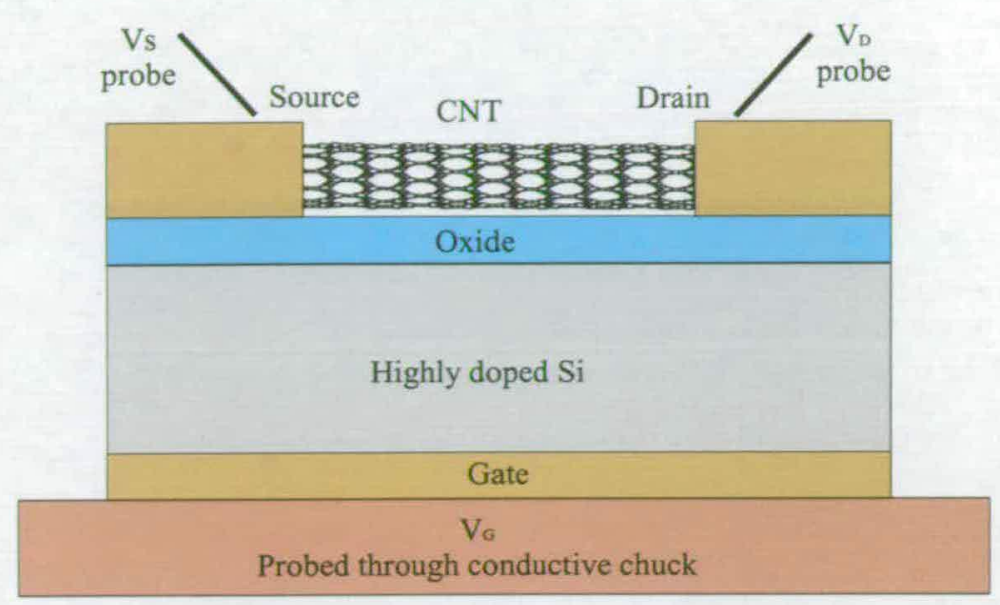

Figure 3.12: The CNT FET probe setup. The source and drain probes are needles points which gently contact the electrode surface. The gate probe is the chuck.

probe, without damaging the electrode. Especially in the case of the needle probes, gentle contact must be achieved. The HP $4156 \mathrm{~B}$ probe analyser allows the outputted CNT device behaviour to be analysed manually in the form of a tex file. The device characteristics have been discussed above in Section 3.3.1 and further characterisation requirements will be made in the results chapters.

\subsubsection{Schottky Diode Characterisation}

The current voltage characteristics of a Schottky diode is usually asymmetrical with high conduction in the forward direction and low conduction in the reverse. Using the data from the current voltage characteristics, parameters can be extracted to determine the dominant mode of conduction and to test the quality of the contact produced. If the diodes fabricated are presumed to adhere to the thermionic emission process, the current voltage (I-V) characteristics can be interpreted by making use of the following method.

As a test of the quality of the Schottky diodes the ideality factor, $\eta$, and the barrier height, $\phi_{B}$, of the Schottky diodes can be obtained by using the thermionic emission theory $[127,131]$. The Schottky diode equation shown below is required.

$$
I=I_{s t}\left[\exp \left(\frac{q V}{\eta k_{B} T}\right)-1\right]
$$


with $\mathrm{I}=$ current and $\mathrm{V}=$ voltage. Here $\mathrm{q}$ is the fundamental unit of charge $1.6 \times 10^{-19} C$, $k_{B}$ is the Boltzmann constant, $1.38 \times 10^{-23} \mathrm{JK}^{-1}, \mathrm{~T}=$ room temperature (300K) and $I_{s t}$ is the saturation current.

$$
I_{s t}=S A^{*} T^{2} \exp \left(-\frac{q \Phi_{B}}{k_{B} T}\right)
$$

where $\mathrm{S}$ is the contact area (the area of the diodes formed), and the Richardson constant $A^{*}$ depends on the semiconductor material. The factor of 1 in equation 3.9 may be ignored as it is only important when $V<\frac{3 k_{B} T}{q}$ otherwise it is negligible.

The extrapolated value of the logarithmic current density as a function of voltage at $\mathrm{V}$ $=0$, gives the saturated current $I_{s t}[127,131]$, using this value, the barrier height can be obtained by

$$
\phi_{B}=\frac{k_{B} T}{q} \ln \left(\frac{S A^{*} T^{2}}{I_{s t}}\right)
$$

which is simply a rearrangement of equation 3.10. The ideality factor $\eta$ is obtained by taking the gradient of the $\frac{\delta V}{\delta \ln (I)}$ slope on the $\ln (\mathrm{I})-\mathrm{V}$ curve, and using equation 3.12 .

$$
\eta \equiv \frac{q}{k_{B} T}\left(\frac{\delta V}{\delta \ln (I)}\right)
$$

However, it has to be considered that ideal diodes are not always observed in experimental work, and often it is necessary to conclude that the current transport is not pure thermionic emission. A hybrid of several conduction methods may be necessary to explain the diode behaviour[133]. It may be the case that there is a leakage current or significant surface defect states which may alter the expected results. In the case of the CNT Schottky contacts, the asymmetrical conditions are difficult to observe as we see two back to back Schottky contacts, which essentially act as a Schottky barrier field effect transistor.

\subsubsection{Field Effect Transistor Characterisation}

As discussed previously in Section 2.2 and 3.3.3, the conduction of CNT FETs is experimentally observed to depend on the Schottky barriers at the CNT electrode interfaces $[53,66,68,73]$, not in a manner completely analagous to.a MOSFET as first thought $[67,72]$. In order to characterise the Schottky barrier field dependent type CNT FETs it is essential to consider how this device varies from the traditional MOS- 
FET. A Schottky barrier type device is dominated by transport through the Schottky barrier, for such a device the assumption of ballistic conduction through the CNT channel will accurately explain the electronic behaviour [53]. Similarly, for short channel (length less than $300 \mathrm{~nm}[53,73]$ ) Ohmic contact CNT devices, conduction is dominated by ballistic conductance. However, it should be noted that the exact nature of the CNT and electrode interface is still not fully understood nor is the exact nature of the conduction in the CNT channel [53]. In a conventional MOSFET the electronic behaviour of the device is dominated by diffusive conductance.

\subsubsection{The subthreshold slope}

A key difference between the Schottky barrier type CNT FET devices and a MOSFET is observed when considering the behaviour of the device before the device turns on when $\mathrm{V}_{g s}<\mathrm{V}_{t h}$, e.g. the switching behaviour of the device. By looking at the subthreshold slope $S[53,68,73,127]$ key differences in the device behaviour are observed.

$$
S=\frac{d V_{g s}}{d\left(\log I_{d}\right)}
$$

In a conventional MOSFET the thermal emission over the conduction or the valence band (for n-type and p-type devices respectively) determines the $I_{d}$ when there is no charge in the channel. There is an exponential dependence of $I_{d}$ on the gate voltage, $V_{g}$, with an exponent proportional to $\frac{1}{k_{B} T}$, so long as $V_{g s}$ is below the threshold voltage $V_{t h}$. In a conventional MOSFET, $S$ only depends on temperature, $T$, and not on any device specific parameters, such as the source drain voltage $V_{d s}$, as there is an Ohmic contact at the source and the drain. In contrast, the short channel CNT FETs with Schottky barrier contacts do depend on device specific parameters as the conduction is dominated by the Schottky barrier. Therefore when $\mathrm{V}_{d s}$ is applied in Schottky barrier CNT FETs a change is induced in $\mathrm{I}_{d}$ through the existing CNT channel. Thus, the subthreshold slope $S$ changes with $V_{d s}$ for CNT devices $[53,68,73,127]$ and does not show a large temperature dependence. 


\subsubsection{CNT mobility}

There is a big change between a conventional MOSFET and a CNT FET in the scaling of the device behaviour. When the channel length of a CNT device $L$, is long in comparison to the gate oxide thickness, $t_{o x}$, the device will be dominated by diffusive conductance, similar to a conventional MOSFET, rather than the ballistic conductance stated for short channel devices above [53]. As discussed above, the conduction behaviour of Schottky barrier CNT FETs is still an interesting topic of study, with the behaviour in the device channel still not fully understood [53]. However, the change from ballistic conductance to diffusive conductance with increasing channel length for a Schottky barrier type CNT device, is generally considered to be $500 \mathrm{~nm}$ and for an Ohmic contact CNT FET device $300 \mathrm{~nm}[53,73]$. For long channel devices it is therefore possible to determine the carrier mobility, $\mu[53,73]$ in the CNT.

$$
\mu=\frac{\sigma}{q}
$$

In a one dimensional conductor the conductivity, $\sigma=G L$, where $G$ is the conductance and $L$ is the length of the devices. The charge density per length of the device, $q=$ $c_{g}\left(V_{t h}-V_{g}\right)$, with $c_{g}$ the capacitance per length, $V_{t h}$ is the device threshold voltage and $V_{g}$ the applied gate voltage. With the channel length significantly greater than the oxide thickness, (which in the case of CNT FETs is a prerequisite to reach the diffusive conductance regime), usually greater than a few micrometers is sufficient to ensure uniform charge density.

$$
\mu=\frac{L}{c_{g}} \frac{G}{\left(V_{t h}-V_{g}\right)}
$$

However, in reality it is often difficult to determine the threshold voltage accurately. Exactly when the device turns on, is often an ambiguous deduction. It is therefore often necessary to consider the field effect mobility, $\mu_{F E}$ which is specific to the individual device rather than the device materials. As such, the field effect mobility also includes the effect of the contacts. 


$$
\mu_{F E}=\frac{L}{c_{g}} \frac{\delta G}{\delta V_{g}}
$$

There is also a further method considering the saturation mobility $\mu_{\text {sat }}$ at high voltages. However, for conventional MOSFETs this method is not well understood [53] and will not be discussed here.

It should also be noted that there is limited information on the mobility of CNTs in the literature due to the difficulty in producing a device with a negligible Schottky contact at the CNT electrode interface. However the intrinsic mobility has been measured at room temperature and observed to be $100000 \mathrm{~cm}^{2} \mathrm{~V}^{-1} \mathrm{~s}^{-1}$ [73]. The CNT FETs made throughout this investigation have not shown saturation and appear to remain in the linear regime. The CNT FETs fabricated are all short channel devices with Schottky barriers and as such, the subthreshold characterisation is the most valid means of analysis. The subthreshold currents will be discussed in the results section along with the implications of the fabrication methods.

\subsubsection{Electronic Device Characterisation Summary}

The HP4156B probe analyser allows the outputted CNT device behaviour to be analysed manually. The electronic probes allow control over the three electrodes fabricated here. Provided there is a clear channel between the source and drain electrodes and the gate oxide is thick enough and of high quality to prevent gate leakage current, the field dependence of the CNTs can be determined.

\subsection{Characterisation Techniques: Conclusion}

The characterisation of carbon nanotubes is possible both chemically and electronically. Spectroscopic techniques such as Raman and x-ray photoelectron spectroscopy have allowed for the pristine and functionalised CNTs structure and chemical attachments to be characterised. Electronic probe techniques using the HP4156B probe analyser have allowed the CNT electronic behaviour to be characterised provided there is a channel between the source and drain electrodes and the CNTs are structurally intact. 


\section{Chapter 4 Plasma Diagnostics and Microfabrication Techniques}

Carbon nanotube field effect transistors (CNT FETs) have been fabricated during this investigation by a combination of chemical functionalisation techniques and self-assembly. The functionalisation of CNTs was achieved by exposure to reactive gases within a standard reactive ion etcher (RIE), a tool which is common to the microfabrication industry. To understand the plasma environment and how functional groups bonded with the CNTs, plasma diagnostic techniques have been applied. The plasma characterisation methods are described in Section 4.1.

In order to connect the nanoscale carbon nanotubes (CNTs) to the macroscopic world, microfabrication techniques have been applied to produce electrode structures, gate oxides, and molecular stamps. Common microfabrication techniques and poly (dimethylsiloxane) (PDMS) stamp fabrication methods are described in Section 4.2.

\subsection{Plasma Diagnostics}

Plasmas are often considered to be the 4th state of matter. In the simplest sense, they are a charged gas containing both ions and neutrals, with high energy electrons causing excitation and ionisation within the plasma. The reactions which take place can be complicated and difficult to analyse, with the exact mechanisms of a plasma process often remaining unknown for the entire plasma process is coupled, and responses to changes of any one parameter can lead to a non-linear change in others. In recent years, the ability to probe inside plasmas has lead to a more detailed understanding of the plasma. An explanation of the physics and chemistry within plasmas can be found in several textbooks $[134,135]$, discussing plasmas for microelectronic processing applications. Discussions of the etch mechanisms, and the advances in the field made from the late seventies and early eighties, are given in several review articles [136-138]. 
A laboratory reactor usually creates a weakly ionised plasma within which the electrons are able to gain far more in kinetic energy than the ions and the neutrals. A schematic representations of a reactive ion etcher (R.I.E.) is shown in figures 4.1 [135].

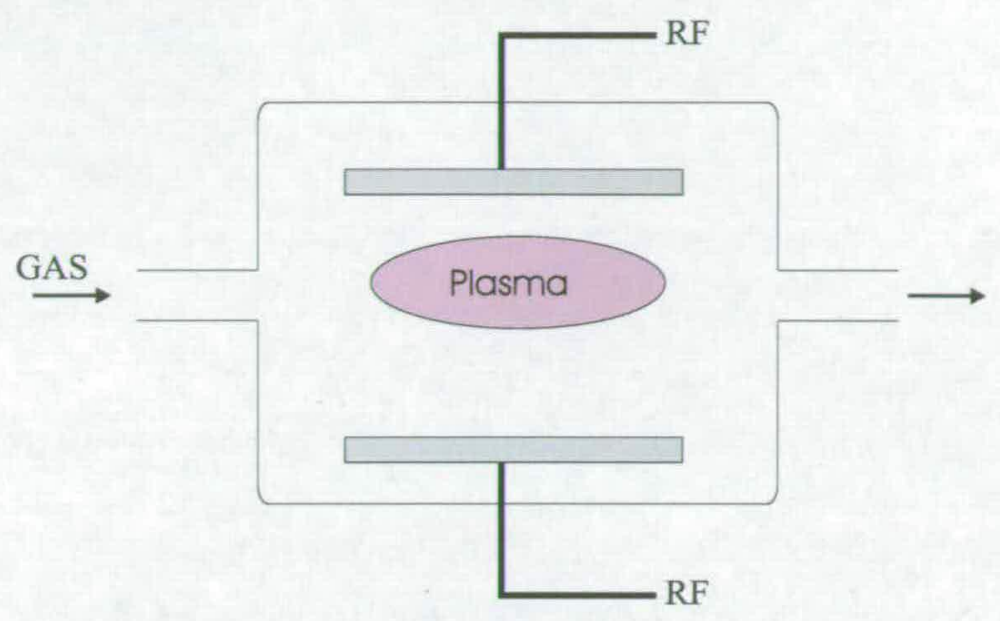

Figure 4.1: A schematic overview of a parallel plate reactor.

The plasma is electrically driven by applying a power to the parallel plates shown in the figure. In doing so, the applied power preferentially heats the electrons rather than the ions. This is because the electrons are considerably lighter and once heated gain far more in kinetic energy than the heavy ions. The power is applied at the industrial frequency of $13.5 \mathrm{MHz}$. During each half wave cycle of the reactor, the fast moving electrons are attracted to the chamber walls. A negative charge then builds up at the chamber wall whilst the plasma becomes positively charged due to the deficit of electrons. Over the next half cycle the ions are attracted to the chamber walls. However, as the ions are slow moving and the frequency of the applied current is so high, there is no significant positive charge built up on the wall before the next half cycle begins. Electrons are again attracted to the chamber walls and the plasma becomes more positive. This continues until an equilibrium has been reached and there is a remaining potential difference between the plasma and the side walls. Between this area of positive and negative charges lies a sheath region.

This is how the ions within the plasma reach a substrate placed within the reactor. The plasma is at a positive potential to all surfaces within the reactor, including any substrates placed on the chuck. The energetic ions are accelerated towards this surface from the plasma. The energy of the ions striking the surface can be controlled 
by altering the bias on the substrate chuck. The potential difference can then be increased by the user. The importance of such ions within the plasma etch system must be considered. Due to the different geometry within plasma systems, and the subsequent changes to the chemical reactions caused by the use of various substrates and gas mixtures, the role of the ions can vary significantly between reactors. There is more than one mechanism defining the plasma etch process, and the complex interactions of the particles within the plasma region and at the surface must be considered for each individual system. The plasma process is discussed in more detail.

Plasmas are formed in modern reactors by applying large electric or magnetic fields across a gas, which then provide the electrons with energy. It is the interaction of the energetic electrons, atoms and the molecules within the gas that cause ionisation and excitation, thus generating the reactive species responsible for the etching to occur. Examples of the electron impact reactions which take place within the plasma are given as follows [137].

1. Excitation (rotational, vibrational, electronic)

$$
e+A_{2} \longrightarrow A_{2}^{*}+e
$$

2. Ionisation

$$
e+A_{2} \longrightarrow A_{2}^{+}+2 e
$$

3. Dissociative Ionisation

$$
e+A_{2} \longrightarrow A^{+}+A+2 e
$$

4. Dissociation

$$
e+A_{2} \longrightarrow 2 A+e
$$


5. Dissociative Attachment

$$
e+A_{2} \longrightarrow=\left\{\begin{array}{l}
A^{-}+A^{+}+e \\
A^{-}+A
\end{array}\right.
$$

Probe diagnostics are crucial to the understanding of plasma functionalisation techniques. Probes are employed in order to understand how the parameters involved in the plasma formation affect any individual reactor. Doing so allows for reproducibility of results and the complexities of the processes involved to be solved. Two probes which are commonly used in plasma diagnostics and were available for this investigation are the Langmuir probe and optical emission spectroscopy (OES). The methods used to extract the relevant information with each of these probes are detailed in this section.

\subsubsection{The Langmuir Probe}

As a simplified approximation, the Langmuir probe consists merely of a wire placed inside the plasma, the workings of such a probe are discussed in review articles and textbooks $[134,135,137,139]$. The probe itself will create a sheath region surrounding itself and as such will locally distort the plasma. The effect of this, however, is considered to be minimal, and is accounted for in the software used to take such measurements [140]. A voltage is applied across the probe and the resulting current is measured. By the direction of the applied bias, either + ve or -ve, the probe will draw upon the electron or the ion current respectively. The Langmuir probe can uncover many of the electrical plasma properties by interpreting the current-voltage (I-V) characteristics of the plasma which are plotted from the probe measurements. Figure 4.2 shows an example of the I-V curves which can be extracted [140] by Langmuir probe measurements.

Here the Smart Probe from Scientific Systems [140] was used. The probe scanned the plasma at various predefined points within the reactor for a set period of time at the etching conditions used during the parameter scans. By varying the voltage across the tip from $-95 \mathrm{~V} \longrightarrow+95 \mathrm{~V}$ and measuring the resulting current, the software provided by Scientific Systems could deduce properties of the plasma in a relatively non-intrusive way. The ion current density was outputted from these measurements and used to determine the role of the ions in the plasma functionalisation process. 


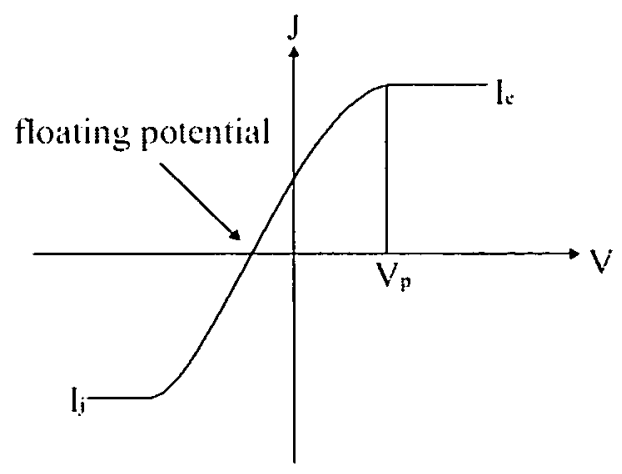

Figure 4.2: A typical example of a Langmuir probe $J-V$ characteristic.

\subsubsection{Optical Emission Spectroscopy}

Optical emission spectroscopy (OES) is a powerful and non intrusive tool, which can be used to study the atomic densities of reactants within the plasma $[134,135,137]$. By using OES here, the concentrations of fluorine within the plasma gas mixtures were calculated. The spectroscopic details of an element are unique and although it is not a trivial matter to distinguish one atom or element from another, this branch of science is well developed and emission spectra information can be accurately linked to the corresponding element or molecule.

The actrinometrical technique used here required the addition of an inert tracer gas to the plasma, in this case the gas used was argon. In order for the actrinomical technique to be successful, the emission of the inert gas must arise from an energy level in close proximity to the excited state giving rise to the species under study [137]. After striking the plasma, the optical emission spectra was read out into a photo multiplier, using the SR 400 Jobin-Yvon double grating spectrometer, enhanced by a GaAs photo multiplier tube, where the data was fed into the relative software package. An example of the emission spectra is shown in figure 4.3

The lines under investigation were argon at $751 \mathrm{~nm}$ and fluorine at $704 \mathrm{~nm}$. The spectroscopic data was read into an Excel file where the areas under the peaks were calculated by integration, the result being the amplitude of the line. The relative fluorine concentrations were then calculated as follows [141]:

$$
F_{\text {pressure }}=\frac{\text { Amplitude of } F_{2}-\text { line }}{\text { Amplitude of } A r-\text { line }} \times\left(P_{\text {plasma }}\right) \times 0.81
$$




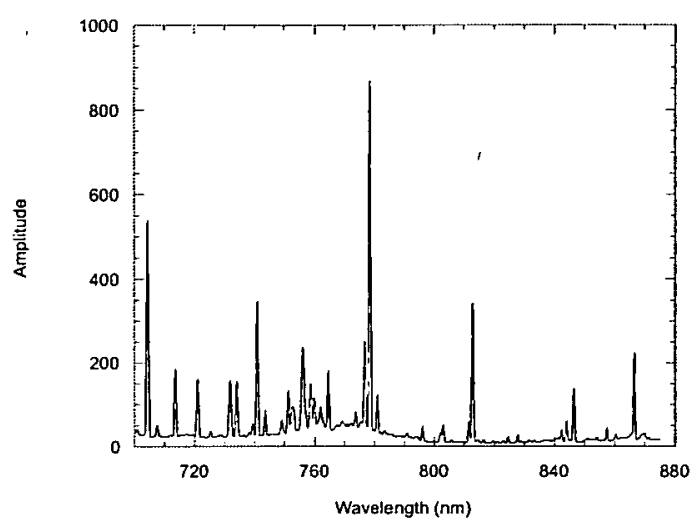

Figure 4.3: An example of the optical emission spectra observed.

where

$$
P_{\text {plamsa }}=\left(P_{\text {plasma }} \text { with } A r\right)-\left(P_{\text {plasma }} \text { without } A r\right)
$$

All of the pressures above were measured in Pascal. The data was used to give an indication of the concentration of the fluorine species present within the plasma during the functionalisation process. Further discussion of the radical species in the plasma will be made in Chapter 5.3.2.

\subsubsection{Plasma Diagnostics: Summary}

A combination of Langmuir probe and optical emission spectroscopy measurement of a plasma environment can characterise the role of the ions and radicals in a plasma system. The plasma diagnostic tools are non-intrusive and effective means of determining how functional groups attach to the CNT sidewalls.

\subsection{Microfabrication}

Standard cleanroom fabrication techniques were employed throughout this work to contact the carbon nanotubes electronically to the macroscopic world for study of their physical behaviour. As the CNTs are inherently on the small scale they are difficult to probe directly without a specialised equipment such as a modified atomic force microscope. A minimum feature size for a contact electrode for manual probing is $100 \mu \mathrm{m}^{2}$, 
which can be produced by either optical or e-beam lithography. The patterning of PDMS stamps used here also required lithographic patterning of a silicon wafer prior to the formation of the stamp itself. The lithographic methods are discussed in the following sections along with the metallisation techniques and standard PDMS stamp fabrication method.

\subsubsection{Electrodes}

The first stage in the fabrication of electrodes required the lithographic patterning on to a substrate surface. Figure 4.4 shows schematically the process flow for patterning the substrate surfaces. The metal is then deposited onto the surface and the excess removed by a process known as lift-off, figure 4.5. The standard procedures for the Lithographic patterning of substrate surfaces are listed below.

\subsubsection{Optical Lithography}

Optical lithography is useful when the minimum feature size to be patterned is greater than $1 \mu \mathrm{m}$ [142]. It is a fast and efficient means of transferring patterns to a wafer surface as usual exposure times are 15 seconds with development times of only a few minutes.

1. A blank silicon or silicon dioxide substrate is cleaned in acetone and IPA with gentle sonication.

2. The substrate is spin coated with resist, SPR2 from Shipley, at $5000 \mathrm{rpm}$ for $40 \mathrm{~s}$. A vacuum syringe was used with a $0.2 \mu \mathrm{m}$ filter to avoid particle contamination.

3. The resist thickness was $1 \mu \mathrm{m}$. The substrates were then soft baked on a hot plate for a minimum of 1 minute at $90^{\circ} \mathrm{C}$.

4. The substrate is exposed to the Cobilt mask aligner using a chromium mask plate for $15 \mathrm{~s}$.

5. The samples are developed for $60 \mathrm{~s}$ by stirring method in 351 developer from Shipley, requiring a 3.5:1 solution of water:developer immediately followed by a stop bath of clean water for 1 minute. 
6. The samples were exposed to a $40 \mathrm{sccm}$ oxygen plasma for $10 \mathrm{~s}$ to produce an undercut.

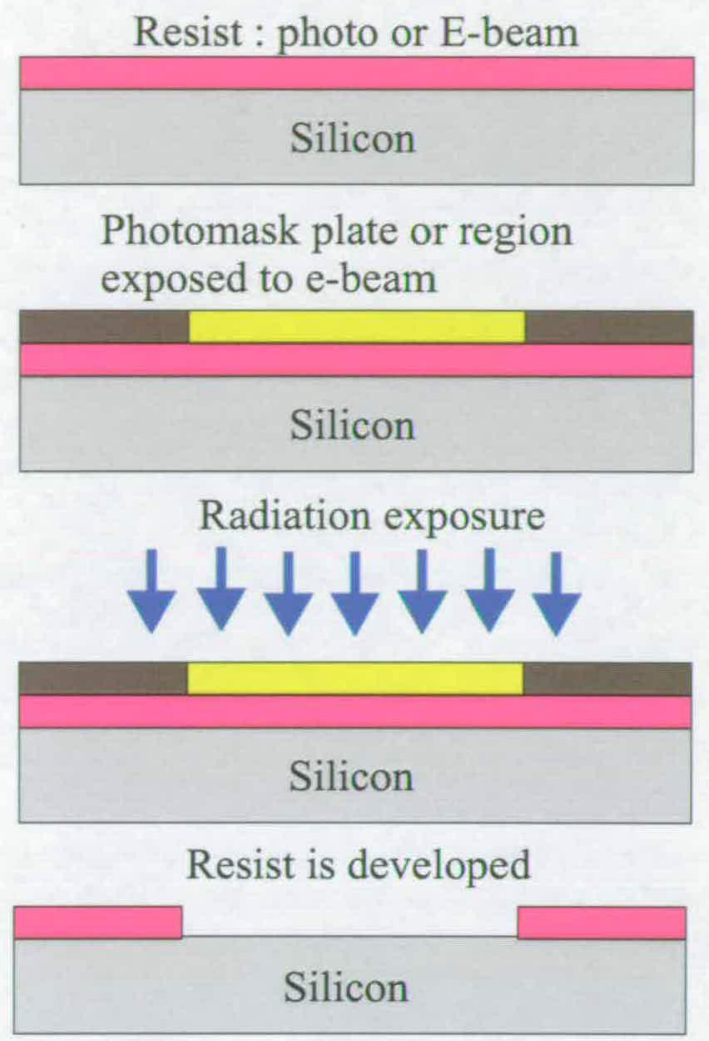

Figure 4.4: The schematic process flow for the lithographic patterning of a substrate

\subsubsection{E-beam Lithography}

E-beam lithography is identical in process to Optical lithography, in the sense that a resist is patterned via exposure to radiation. As such figure 4.4 and 4.5 are also valid descriptions for the E-beam lithography process. E-beam exposure becomes essential when the minimum feature size to be patterned is less than $1 \mu \mathrm{m}[142]^{1}$. In contrast to optical lithography the E-beam exposure process is the time limiting step as the exposure takes place via a single electron beam scanning over a predefined field size, which can at times be less than $100 \mu \mathrm{m}^{2}$. The E-beam must scan over the entire chip or wafer, which for a 4 inch wafer results in many fields. The pattern, analogous to the

\footnotetext{
${ }^{1}$ In most university cleanroom facilities. However, it should be noted that recent advances in Lithography common to industry fabrication centres can produces submicron features as standard with optical lithogrphy techniques.
} 
mask in the optical process, is fed into the e-beam writer by means of an electronic file, usually in GDSII format. The beam then scans and exposes according to the pattern instructions.

1. A blank silicon or silicon dioxide substrate is cleaned in acetone and IPA with gentle sonication.

2. The substrate is spin coated with PMMA resist at $5000 \mathrm{rpm}$ for $40 \mathrm{~s}$. A vacuum syringe can be used but is not always essential as often e-beam resists are thicker than their optical counterparts.

3. The resist thickness was $1 \mu \mathrm{m}$. The substrates were then baked in an oven in inert $\mathrm{N}_{2}$ atmosphere for a minimum of 1 hour at $170^{\circ} \mathrm{C}$.

4. The substrate is exposed to the E-beam. Here the Joel JBX-5FE was used.

5. The samples are developed for 60s in an MIBK:IPA mixture, (1:3) ratio before being placed in a stop bath of IPA.

\subsubsection{Metallisation}

To make the final electrodes the patterned substrate is placed into a metal deposition chamber, either an evaporator or sputterer. Evaporation can be split into two main divisions, thermal and e-beam. A thermal evaporator works by applying a large current across a tungsten filament containing the electrode metal to be deposited, thermally heating a metal. Likewise, the e-beam evaporator uses an electron gun fired at a metal target, to heat the metal. Both evaporation methods result in metals becoming so hot that they vapourise. The evaporated metals will strike the surface of the sample at close to $90^{\circ}$ if the sample positioning is correct, see figure 4.5 , making metal lift-off processes possible.

Sputtering methods are slightly different in the sense that a metal target is bombarded with energetic ions causing the metal ions to bounce off the source. In such a situation the metal ions strike the sample surface in all directions making metal-lift-off processes difficult to achieve. Sputtering is therefore more suitable to producing back Ohmic contacts, or a blanket metal layer which can be patterned by etching over the metal. 

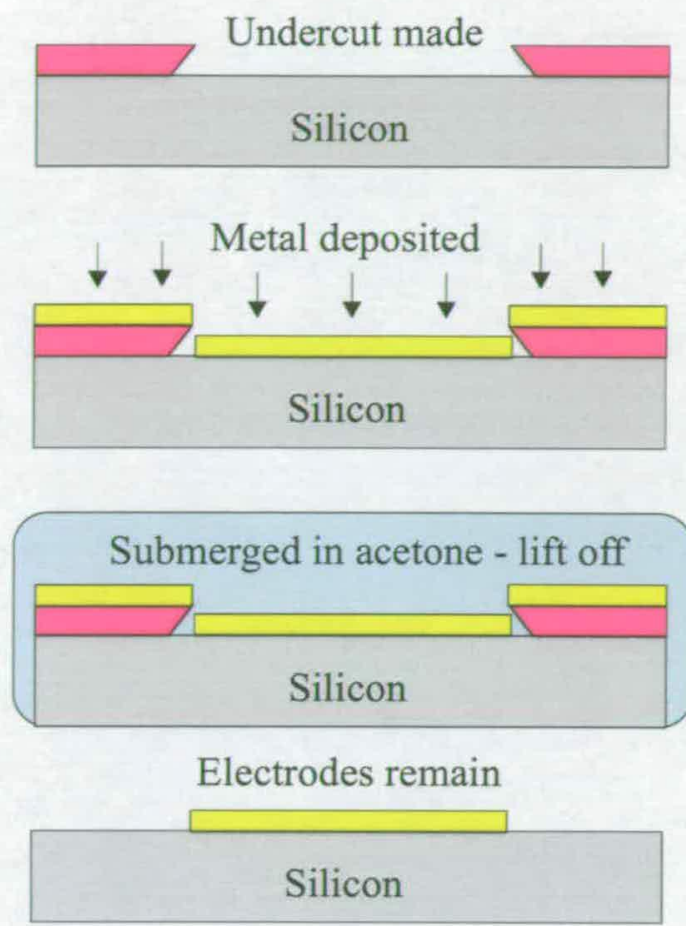

Figure 4.5: The schematic process flow for metalisation

Listed below are the main steps in metallisation used here, where lift-off has been achieved:

1. A lithographically patterned substrate is placed into the evaporator. Preferably the resist has a slight undercut.

2. The metal is deposited at right angles (approximately) to the substrate surface.

3. The substrate is submerged in acetone to dissolve the resist; ultrasonication can be performed if required.

4. The substrate is then cleaned in acetone and IPA. The metal above the resist has been removed by the acetone. Only the metal making contact with the substrate surface is left.

Several considerations are needed in the metallisation process. For instance, thermal evaporation is only possible for metals with a relatively low evaporation point, such as aluminium, gold and nichrome, as there are usually limits to the current driving abilities of the evaporator, preventing sufficient heat to be created. Also, it is difficult to 
stick gold to $\mathrm{SiO}_{2}$ substrates, and as such a sticking layer, usually chromium or titanium is required.

In the final stage of lift-off where sonication can be applied to assist the acetone in the resist dissolving stage, care must be taken to avoid ragged edges of the electrodes as the sonication can sometimes be too aggressive. However, metal lift-off techniques are relatively simple, fast and effective ways of creating high quality contacts for CNT electronic characterisation.

\subsubsection{PDMS Stamps}

Molecular stamping techniques have been applied for the self-assembly of carbon nanotubes. Poly(dimethylsiloxane) (PDMS) stamps have been fabricated following similar procedures to those in the literature $[40,41]$, shown schematically in figure 4.6 . To produce a PDMS stamp a silicon wafer was originally patterned by lithography as shown for the electrode fabrication in Section 4.2.1.

Lithographically patterned resist

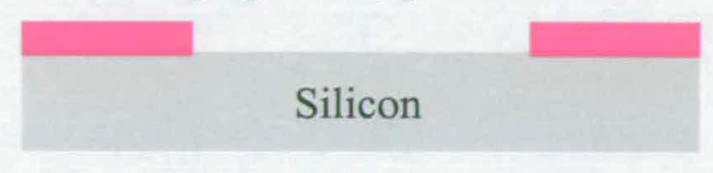

TMAH etched Silicon

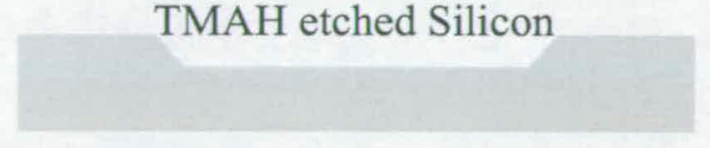

Fluorocarbon layer
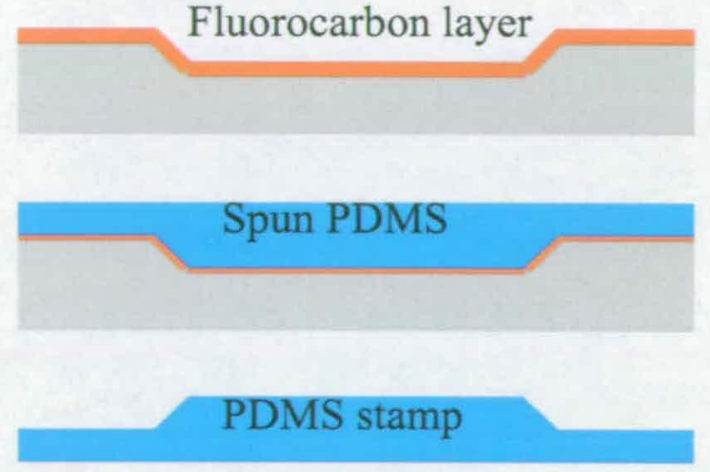

Figure 4.6: The schematic formation of a PDMS stamp

1. A lithographically patterned substrate is etched with TMAH to produce a smooth 
sloped sidewall etch profile.

2. A fluorocarbon layer was deposited by spin coating over the etched substrate.

3. The degassed PDMS was spin coated over the wafer at $100 \mathrm{rpm}$.

4. The stamps were then cured at $120^{\circ} \mathrm{C}$ for at least 2 hours.

5. The PDMS stamp can then be easily removed from the wafer and cut via a knife to the required size for the molecular stamping.

The PDMS used was the commercially available Sylgard 184. The base and curing agent were mixed together thoroughly at a weight ratio of 10:1 respectively $[40,41$, $143,144]$. After mixing the PDMS was placed in a vacuum chamber and completely degassed. The fluorocarbon layer prevents the PDMS stamp from sticking to the wafer surface and as such reduced the number of defects found on the stamp surface after removal.

\subsubsection{Microfabrication Techniques: Summary}

The microfabrication techniques described here have been critical for the probing of CNT electronic behaviour. Although the techniques described are not conceptually complex, it is necessary to appreciate that these techniques have been refined and developed over several years. In any experimental procedure reproducibility is crucial for successful device production, as such the described techniques are excellent for the CNT device characterisation as the procedures are well developed and known to be highly reproducible.

All techniques described are standard within the semiconductor device industry and research fields and are performed in a cleanroom environment to minimise contamination and increase reproducibility. As such it is essential to make CNT device structures which are compatible with such standard techniques for expedient integration into current technologies. 


\section{Chapter 5 Plasma Fluorination of Carbon Nanotubes}

As discussed in the introduction, carbon nanotubes (CNTs or SWNTs) have been functionalised by several groups to improve suspension and to control the electronic behaviour. However, one of the problems with some functionalisation methods was the damage caused to the CNT structure, as well as the length of time required for the functionalisation procedure to take place $[8-10,34]$.

In the following chapter a CNT functionalisation process has been developed by short time exposure to fluorine based plasmas. An emphasis is given to the control of the fluorination process used here as it is hypothesised that functionalisation will create a chemical interaction between the CNTs and a functional attachment, thereby allowing control of the electronic properties of the CNTs.

Fluorine is a useful functionalisation element for carbon nanotubes due to the high electronegativity making the $\mathrm{C}-\mathrm{F}$ bond easy to achieve and also strong and stable. The high reactivity also makes fluorine useful for further manipulation, as the correct choice of reaction agents can be used to substitute fluorine for other molecules. In Chapter 6, plasma fluorinated CNTs will be further functionalised with amine containing molecules. The electronic characterisation of plasma fluorinated CNTs will be compared to the amine functionalised CNTs in Chapter 6. In the meantime the following chapter will discuss the fluorination of CNTs and the mechanism of attachment in the plasma environment.

\subsection{The Fluorination of Carbon Nanotubes, a Brief Review}

A thorough review of CNT fluorination procedures up to the start of this investigation is given by Valery [8]. The highlights involved the method proposed by Mickelson showing 
control on the resulting $\mathrm{C}_{x} \mathrm{~F}_{y}$ stoichiometries by altering reaction time and temperature. The fluorination process was seen to be reversible by exposure to hydrazine [10]. The nature of the fluorination on the CNTs was studied by Kelly et al. [145] where Scanning Tunnelling Microscopy (STM) had shown sharp bands of CNT-fluorination regions.

Two mechanisms of the fluorination were observed, the 1,4-isomer, where the fluorine bonds to the carbon at positions 1 and 4 on the benzene ring, and the 1,2-isomer, similarly explained. The known stoichiometry control and the band forming fluorination structure was later exploited by $\mathrm{Gu}$ et al. to produce F-CNTs at $\mathrm{CF}_{0.2}$ stoichiometry. These F-CNTs were then pyrolyzed in argon removing the $\mathrm{F}$ as $\mathrm{CF}_{4}$ and $\mathrm{COF}_{2}$ (confirmed by in-situ FT-IR), leaving reduced length CNTs, i.e. the CNTs were cut in a controlled fashion. The fluorination is seen to be useful for improving the suspensions of CNTs and controlling the length. However, the functionalisation processes can also be damaging to the CNT structure, whereby at stoichiometry $\mathrm{C}_{2} \mathrm{~F}$, CNTs are known to break down structurally [10].

\subsubsection{Plasma Fluorination of Carbon Nanotubes}

The methods employed here involved plasma functionalisation of CNTs usually occurring in several minutes and with limited damage to the structure of the nanotubes. The initial motivation for the plasma fluorination came from the observation in traditional plasma etching process used in the microfabrication of electronic devices, plasma exposure would often result in a fluorine rich carbon film on substrate surfaces $[117,146]$.

The fluorination of CNTs with a plasma began firstly with use of a $\mathrm{CF}_{4}$ plasma using a Vacutec reactive ion etching system. The use of a $\mathrm{CF}_{4}$ plasma as a functionalisation process for CNTs has become more common since the publication of our initial study $[17,18]$ and several authors have found similar functionalisation achievements using such a plasma $[11,13]$. Other plasma functionalisation methods have also been developed, namely by Khare et al. $[147,148]$, where $\mathrm{H}_{2}$ groups were attached to CNTs by plasma exposure. 


\subsection{Carbon Nanotubes Functionalised in a $\mathrm{CF}_{4}$ Plasma}

\subsubsection{Experimental Setup of Fluorinated Plasmas}

Single wall carbon nanotubes, (CNTs), from Carbon Nanotechnologies Inc, were prepared by dispersion in isopropanol (IPA), at a concentration of $5 \mathrm{mg}$ of CNTs to $15 \mathrm{ml}$ of IPA, using a magnetic stirrer for 2 hours at room temperature resulting in the formation of a thick black paste. The dispersion was then filtered through a $2 \mu \mathrm{m}$ polytetrafluoroethylene (PTFE) filter membrane from Millipore under vacuum and left to dry for 24 hours at room temperature, resulting in the formation of bucky papers on top of the PTFE membranes. Subsequently, the samples were exposed to $\mathrm{CF}_{4}$ plasmas with 20 and $40 \mathrm{sccm}$ flow rates for $30 \mathrm{~s}, 1$ and 2 minutes in a Vacutec reactive ion etcher (R.I.E), with a chamber pressure of $0.15 \mathrm{~Pa}$ at $20 \mathrm{sccm}$ and $0.196 \mathrm{~Pa}$ at $40 \mathrm{sccm}$. All samples were exposed to the plasma with a DC bias of $300 \mathrm{~V}$.

A comparison was then made between a $\mathrm{CF}_{4}$ plasma and an $\mathrm{SF}_{6}$ plasma as a means of functionalisation at various reactor conditions outlined in Table 5.2. The plasma conditions were characterised with optical emission spectroscopy (OES) and Langmuir probe measurements, as described in Chapter 4. The mechanism of fluorine attachment to the CNTs is discussed. To characterise the CNTs after exposure to a plasma, Raman spectroscopy was initially used. The Raman spectra of carbon nanotubes is known to have a distinctive shape as discussed in Chapter 3.1 and was therefore a useful first step to assess whether the plasma environment was too aggressive for the tubes to remain intact.

\subsection{2 $\mathrm{CF}_{4}$ Plasma Exposed CNTs: The Raman Spectra}

The Raman spectra of the control and $\mathrm{CF}_{4}$ plasma exposed carbon nanotubes (CNTs) are shown in figure 5.1. The shape of the spectra remains unchanged throughout the parameter scans with the intensity of the lines being altered. Both the $\mathrm{G}$-line (at around $1580 \mathrm{~cm}^{-1}$ ) corresponding to $\mathrm{sp}^{2}$ bonded carbon, characteristic of the $\mathrm{C}-\mathrm{C}$ bond in carbon nanotubes and the D-line (at around $1375 \mathrm{~cm}^{-1}$ ) corresponding to $\mathrm{sp}^{3}$ bonding, relating to scattering defects in the CNT samples are evident in the spectra.

The data shows strong agreement with previously reported Raman studies of CNTs [121, 


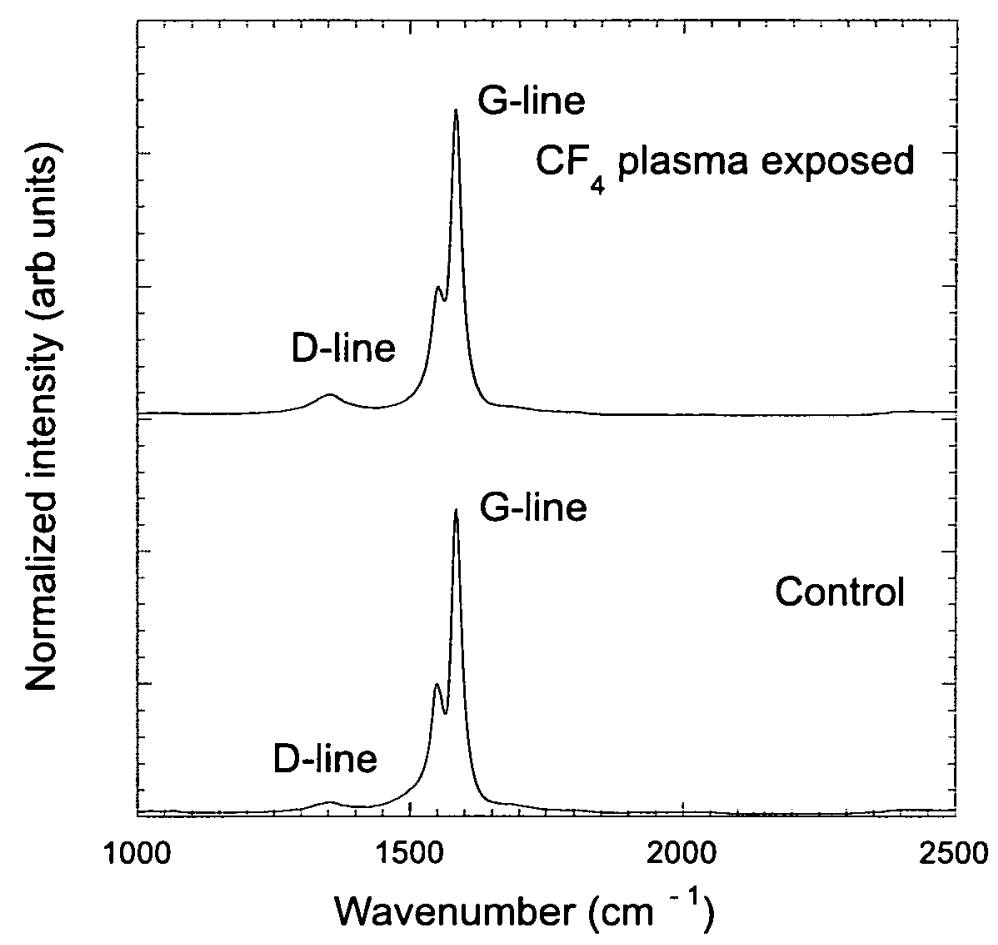

Figure 5.1: The Raman spectra of a typical control and a typical $C F_{4}$ plasma exposed $C N T$ samples

149-152]. Furthermore, the Raman spectra are characteristic of CNTs when compared with the Raman spectra of amorphous carbon, glassy carbon and carbon black [149].

\subsubsection{The D/G Ratio}

The relative intensity ratios of the D to G-bands shown in figure 5.2 give an indication of the degree of disorder in the carbon nanotubes. The weak $\mathrm{D}$ band at approximately $1300 \mathrm{~cm}^{-1}$ has previously been correlated to the extent of sidewall functionalisation [121], it is highly likely that the same effect occurs on our samples. The general trend is that as the flow rate of the gas increases, a larger degree of disorder, functionalisation is observed. Moreover, exposing the carbon nanotubes to $\mathrm{CF}_{4}$ plasma for 2 minutes results in a larger degree of disorder compared to exposure for 1 minute. It should be noted here that the G-band has stayed strong throughout indicating that a large proportion of ordered nanotubes remain.

It has been reported that energies up to $100 \mathrm{keV}$ are necessary to damage the tubes [153] and at $300 \mathrm{eV}$, the energies involved in the plasma process, the nanotubes would not 


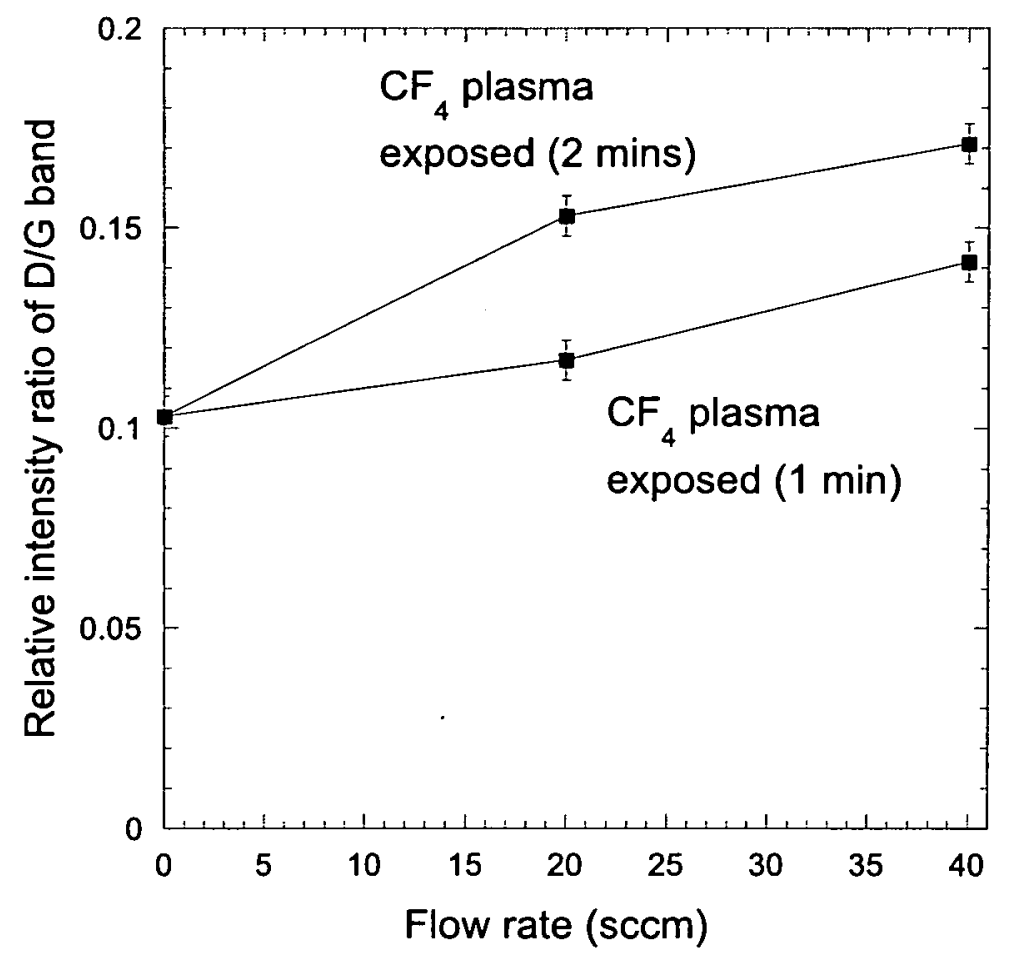

Figure 5.2: The ratio of the $D$-band to the $G$-band as a function of $C F_{4}$ plasma exposure conditions. Over all conditions the D.C. bias was held at $-300 \mathrm{~V}$

have been damaged although they may be slightly modified. The processes involving either fluorine or oxygen attachment in other work has caused the Raman spectra to alter significantly $[9,10,118,121]$, with broadening of the $D$ and $G$ peaks.

\subsubsection{Scanning Electron Microscopy of the CNT Surface}

In these experiments, due to the short reaction times and the reaction mechanism, plasma exposure appears to be a fast and reliable method of fluorinating CNTs without loss of structure. SEM images of the control sample and the $\mathrm{CF}_{4}$ plasma functionalised CNTs is shown in figure 5.3. Little difference is observed between the two samples further indicating that little damage has been caused to the CNT surfaces.

\subsection{5 $\mathrm{CF}_{4}$ Plasma Exposed CNTs: The XPS Spectra}

The presence of fluorine on the carbon nanotube surfaces was investigated by $x$-ray photoelectron spectroscopy (XPS) as described in Chapter 3.2. Emphasis has been placed 


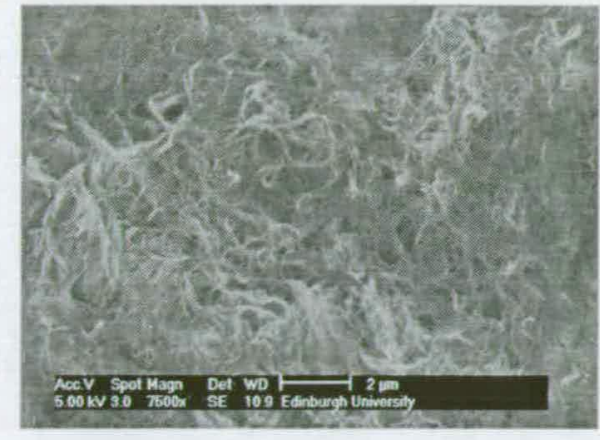

(a) SEM images of the control bucky paper

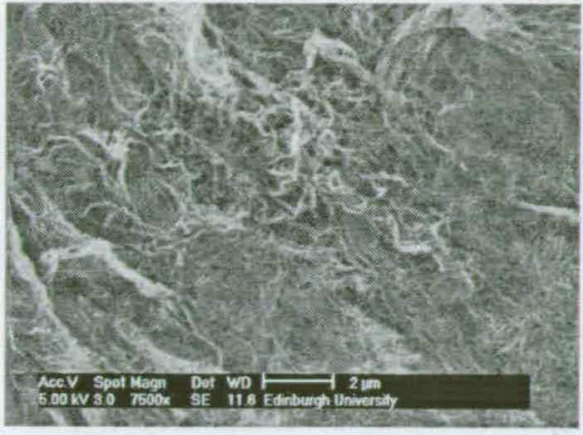

(b) SEM image of the $\mathrm{CF}_{4}$ plasma exposed bucky paper

Figure 5.3: $S E M$ images of typical control and $\mathrm{CF}_{4}$ plasma exposed samples

on the F1s peaks although the $\mathrm{C} 1 \mathrm{~s}$ and $\mathrm{O} 1 \mathrm{~s}$ peaks have also been recorded. Due to the nature of the carbon and oxygen peaks it is difficult to determine exactly how a useful and valid deconvolution would be carried out as both of these elements can be due to atmospheric contaminants. The deconvolution of the $\mathrm{C} 1 \mathrm{~s}$ peak is also further complicated by the overlapping binding energies of the $\mathrm{C}_{x} \mathrm{~F}_{y}$ and $\mathrm{C}_{m} \mathrm{O}_{n}$ species. The fluorine present on the CNTs is only possible due to the plasma exposure, therefore making the F1s peak a valid means of analysis.

\subsubsection{Deconvolution of the XPS Spectra: The C1s Region}

All the core level spectra of the plasma exposed samples have been analysed by deconvoluting with Gaussian peaks after subtraction of linear mode backgrounds. Figure 5.4 and 5.5 show the normalised $\mathrm{C} 1 \mathrm{~s}$ and $\mathrm{F} 1 \mathrm{~s}$ spectra of the control and $\mathrm{CF}_{4}$ plasma exposed tubes.

From figure 5.4, it can be seen that the signals from the $\mathrm{sp}^{2} \mathrm{C}-\mathrm{C}$ bond corresponding to CNTs dominate the C1s spectra in the control and plasma exposed tubes. The control sample spectra observed is similar to those found for pristine nanotubes by other authors $[90,118]$. A slight broadening is observed at the higher binding energies for the $\mathrm{CF}_{4}$ plasma exposed sample. Because of the presence of fluorine detected on our $\mathrm{CF}_{4}$ plasma exposed nanotubes, see figure 5.5 , the high energy peaks in the $\mathrm{C} 1 \mathrm{~s}$ spectra are thought to be mainly due to fluorinated carbon rather than to $\mathrm{C}=\mathrm{O}$ and $\mathrm{O}-\mathrm{C}=\mathrm{O}[9,10,118,121]$; due to the known strong electronegativity of fluorine a C-F 


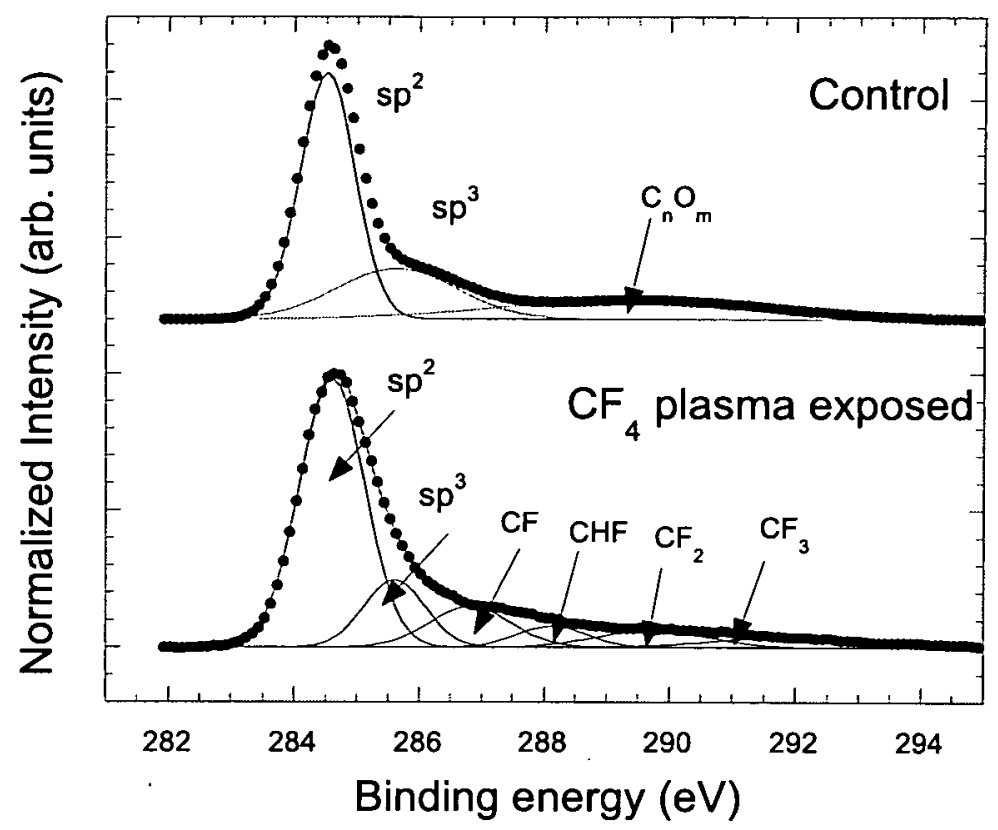

Figure 5.4: The C1s peaks of the control sample and the $C F_{4}$ plasma exposed sample

bond is thought to be more favourable to a $\mathrm{C}-\mathrm{O}$ bond ${ }^{1}$. However, due to the overlapping energies it is non-trivial to distinguish which peaks belong to fluorinated or oxygenated carbon species.

Apart from the main C1s component peak at $284.6 \mathrm{eV}$, there is the $\mathrm{sp}^{3} \mathrm{C}-\mathrm{C}$ [121] Gaussian peak at $285.6 \mathrm{eV}$ as well as peaks observed at $287.1 \mathrm{eV}, 289.8 \mathrm{eV}$ and at $292.4 \mathrm{eV}$ which may correspond to $\mathrm{CF}, \mathrm{CF}_{2}, \mathrm{CF}_{3}[9]$ or $\mathrm{C}-\mathrm{O}, \mathrm{C}=\mathrm{O}$ and $\mathrm{O}-\mathrm{C}=\mathrm{O}[118,121]$. The peak observed at $288 \mathrm{eV}$ may also be due to a contribution from the CHF bonds [114], or have arisen due to $\mathrm{C}_{2} \mathrm{~F}$ bonding [119], or indeed, be a combination of both. The $\mathrm{C} 1 \mathrm{~s}$ spectra of the control sample may be similarly deconvoluted. The $\mathrm{sp}^{2}$ and $\mathrm{sp}^{3}$ carbon will be present along with components from oxygenated species at high energy.

\subsubsection{Deconvolution of the XPS Spectra: The F1s Region}

The normalised F1s peaks, figure 5.5, show the presence of fluorine on the bucky papers is only detected on the $\mathrm{CF}_{4}$ plasma exposed tubes. The nature of the carbon to fluorine bonds (C-F) has been analysed by deconvoluting the $\mathrm{F} 1 \mathrm{~s}$ peak of the $\mathrm{CF}_{4}$ plasma

\footnotetext{
${ }^{1}$ Evidence of this effect has been observed whereby CNTs were exposed to an $\mathrm{O}_{2}$ plasma in the Vacutec RIE. As the reaction chamber was not sufficiently clean residual F-species were observed. This is discussed later in Section 5.3.3.
} 
exposed CNTs.

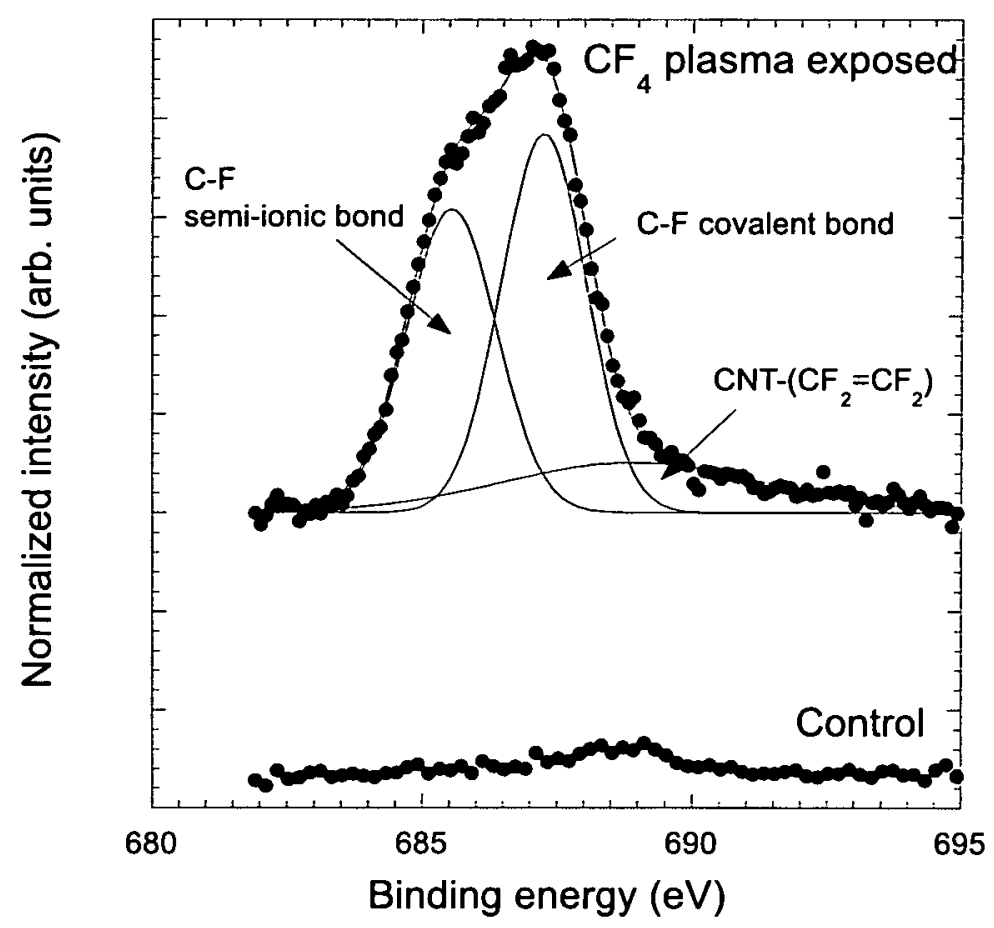

Figure 5.5: The F1s peaks of the control sample and the $\mathrm{CF}_{4}$ plasma exposed sample

Both semi-ionic (at $685.5 \mathrm{eV}$ ) and covalent (at $687.5 \mathrm{eV}$ ) C-F bonds [117, 119, 154], have been clearly observed. There is also a third peak observed in the F1s spectra of the $\mathrm{CF}_{4}$ plasma exposed nanotubes at $689.2 \mathrm{eV}$, possibly due to the presence of $\mathrm{p}\left(\mathrm{CF}_{2}=\mathrm{CF}_{2}\right)$ bonds [114]. The occurrence of the fluorinated carbon bonds is believed to be due to the $\mathrm{CF}_{4}$ plasma chemistry [155-157].

\subsubsection{The Semi-ionic and Covalent F1s Regions}

As it is difficult to determine the precise contribution from different types of carbon fluorine bonds, e.g. the $\mathrm{CF}_{2}$ binding energy is very close to $\mathrm{CF}_{3}$ in the F1s spectra [119], a useful means of characterising the $\mathrm{F} 1 \mathrm{~s}$ region is to calculate the ratio of the semi-ionic and covalent contributions. The intensity ratios of semi-ionic and covalent C-F bonds $\left(\mathrm{I}_{\text {semi-ionic }} / \mathrm{I}_{\text {covalent }}\right.$ ) from the $\mathrm{CF}_{4}$ plasma exposed CNTs are shown in Table 5.1.

It is noticed that the presence of covalently bonded C-F increases as the $\mathrm{CF}_{4}$ gas flow rate is increased during $\mathrm{CF}_{4}$ plasma exposure and/or with increase of exposure time. The smallest ratio is observed for the highest $\mathrm{CF}_{4}$ flow rate and maximum exposure time 


\begin{tabular}{|ccc|}
\hline Flow rate & $20 \mathrm{sccm}$ & $40 \mathrm{sccm}$ \\
\hline time (min) & & \\
1 & 0.888 & 0.848 \\
2 & 0.810 & 0.639 \\
\hline
\end{tabular}

Table 5.1: $I_{\text {semi-ionid }} / I_{\text {covalent }}$ intensity ratio for the $C-F$ bonds

under study. This observation is commensurate with the fact that covalently bonded C-F occurs when the localisation of $\pi$ electrons of the $\mathrm{C}$ atoms increases strongly as a result of an increase in the $\mathrm{F}$ density, causing the $\mathrm{F}$ atoms to get closer to the $\mathrm{C}$ atoms [118, 119]. The position of the F1s peak will be discussed again later in Section 5.3.3.

It should be noted that the F1s and C1s peaks observed during this study are similar to those found during the fluorination of CNTs by An et al. [118] at low temperatures $\left(150^{\circ} \mathrm{C}\right)$ where the $\mathrm{CNTs}$ had an $\mathrm{F} / \mathrm{C}$ ratio $<0.5$. The highest $\mathrm{F} / \mathrm{C}$ ratio found in our $\mathrm{CF}_{4}$ plasma exposed CNTs has been 0.22 , which is less than the theoretical and experimental saturation stoichiometries of $\mathrm{C}_{2} \mathrm{~F}$ [10] or $\mathrm{CF}_{0.5}$ [118], thus indicating the possibility that the $\mathrm{F}$ atoms are chemisorbed at the outer surface of the CNT wall $[118,119]$. Here a level of control in the fluorination procedure has been achieved by altering the conditions of the plasma exposure. Due to the low stoichiometries and preservation of the structure of the CNTs observed it is possible that during the short plasma exposure, only the reactive sites, or defect sites, of the CNTs are functionalised.

\subsubsection{Summary: $\mathrm{CF}_{4}$ Plasma Functionalisation}

In summary, the effect of changing $\mathrm{CF}_{4}$ plasma exposure parameters and the resulting changes to the single-wall carbon nanotubes has been studied. Raman spectroscopy results indicate that the modifications to the CNTs structure have been subtle and that the structural integrity of the tubes has been maintained. The macroscopic appearance of the CNTs before and after plasma exposure, are shown to be similar from the SEM images.

X-ray photoelectron spectroscopy has shown that fluorine can be attached to CNTs using 
a $\mathrm{CF}_{4}$ plasma. The intensity ratio of $\mathrm{I}_{\text {semi-ionic }} / \mathrm{I}_{\text {covalent }}$ of the $\mathrm{F} 1 \mathrm{~s}$ peak is seen to decrease upon the increase of flow rate of the plasma gas and exposure time of the samples to the $\mathrm{CF}_{4}$ plasma, showing that the fluorination process is sensitive to the reactor conditions. The low $\mathrm{F} / \mathrm{C}$ ratios of the CNTs confirm that the fluorination process has not yet reached saturation.

The results indicate that exposing CNTs to a short plasma exposure process can cause functionalisation of the carbon nanotubes without damaging the unique CNT structure.

\subsection{The Comparison of $\mathrm{CF}_{4}$ and $\mathrm{SF}_{6}$ Plasma}

The comparison between a $\mathrm{CF}_{4}$ plasma and an $\mathrm{SF}_{6}$ plasma as a means of functionalisation was achieved by studying the CNTs exposed to various reactor conditions outlined in Table 5.2. Bucky papers were prepared as described in the experimental Section 5.2.1. The mechanism of the fluorine attachment is studied by a comparison of the CNTs surface using XPS and Raman spectroscopy and the characterisation of the plasma generated at the different reactor conditions, using both optical emission spectroscopy (OES) and Langmuir probe measurements discussed in Chapter 4.1.

It should be noted that the flow of the $\mathrm{SF}_{6}$ gas is kept to $20 \mathrm{sccm}$ as opposed to 40 sccm of the $\mathrm{CF}_{4}$ plasma. Due to the heaviness of $\mathrm{SF}_{6}$ gas a higher R.F. power is need to dissociate the feed stock as a successful plasma mixture. The matching of the system is critical in order to effectively and accurately characterise the reactor behaviour. As such at a flow rate of $20 \mathrm{sccm} \mathrm{SF}_{6}$ the plasma environment is similar to that of the $\mathrm{CF}_{4}$ plasma at $40 \mathrm{sccm}$ with power required and measured bias voltages.

\subsection{1 $\mathrm{CF}_{4}$ and $\mathrm{SF}_{6}$ Plasma Exposed CNTs: The XPS Spectra}

The $\mathrm{F} 1 \mathrm{~s}$ region of $\mathrm{CNTS}$ exposed to the $\mathrm{CF}_{4}$ plasma, the $\mathrm{SF}_{6}$ plasma and the unexposed control sample are shown in figure 5.6. The F1s peak of the CNTs functionalised in the $\mathrm{CF}_{4}$ plasma and the $\mathrm{SF}_{6}$ plasma have been deconvoluted using XPS software. The tubes functionalised with $\mathrm{CF}_{4}$ plasma require two large Gaussian peaks to obtain a good fit with the measured data, one at $686 \mathrm{eV}$ in the semi-ionic region and another at 687.5 $\mathrm{eV}[114,119]$ in the covalent C-F bonding region, over all exposure conditions. 


\begin{tabular}{|ccc|ccc|}
\hline \hline & \multicolumn{2}{c|}{$\mathrm{CF}_{4}$} & \multicolumn{3}{|c|}{$\mathrm{SF}_{6}$} \\
Flow (sccm) & Bias voltage $(\mathrm{V})$ & Time $(\mathrm{s})$ & Flow $(\mathrm{sccm})$ & Bias voltage $(\mathrm{V})$ & Time $(\mathrm{s})$ \\
\hline 10 & -200 & 60 & 10 & -200 & 60 \\
20 & -200 & 60 & 20 & -200 & 60 \\
30 & -200 & 60 & 30 & -200 & 60 \\
40 & -200 & 60 & & & 60 \\
\hline 40 & -0 & 60 & 20 & -0 & 60 \\
40 & -300 & 60 & 20 & -300 & 60 \\
40 & -400 & 60 & 20 & -350 & 30 \\
\hline 40 & -200 & 30 & 20 & -200 & 120 \\
40 & -200 & 120 & 20 & -200 & \\
\hline 40 & -200 & 180 & & & \\
\hline
\end{tabular}

Table 5.2: Reactor conditions of the Vacutec RIE system.

The CNTs functionalised in $\mathrm{SF}_{6}$ plasma however need only one large peak at $687 \mathrm{eV}$, towards the covalent region of the F1s spectra to fit the measured data, again over all exposure conditions. The broad low intensity peak at $689.5 \mathrm{eV}$ is present after both functionalisation procedures and is thought to be due to weak sputtering effects within the plasma environment.

\subsection{2 $\mathrm{CF}_{4}$ and $\mathrm{SF}_{6}$ Plasma Exposed CNTs: The Attachment Mechanism}

The possible mechanism for this behaviour has been studied using OES and Langmuir probe measurements. The results from the OES have been used to calculate the fluorine pressure within the reactor using an actinometry technique described previously in Chapter 4.1. 


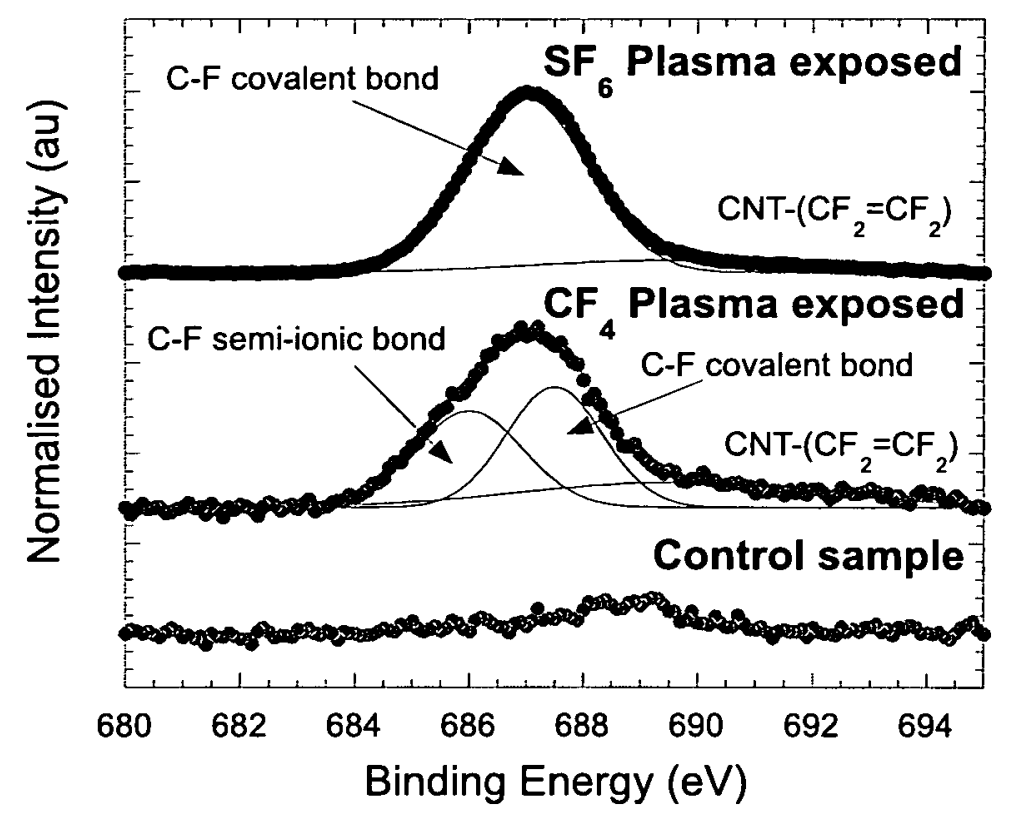

Figure 5.6: Typical $F 1 s$ peaks of the control, $\mathrm{CF}_{4}$ exposed and $S F_{6}$ exposed samples

\subsubsection{Plasma Diagnostics}

The fluorine pressure as a function of bias voltage is shown in figure 5.7(a). The result at $0 \mathrm{~V}$ bias refers to only the flow of $\mathrm{SF}_{6}$ and $\mathrm{CF}_{4}$ process gases respectively, as the plasma environment could not be maintained without application of a bias voltage; therefore no fluorine radicals are produced.

The OES results show that there is a higher concentration of fluorine within the $\mathrm{SF}_{6}$ plasma than the $\mathrm{CF}_{4}$ plasma over all conditions, even though the actual flow rate is $20 \mathrm{sccm}$ for the $\mathrm{SF}_{6}$ gas and $40 \mathrm{sccm}$ of $\mathrm{CF}_{4}$. The fluorine pressure increases by $7.32 \times 10^{-4} \mathrm{~Pa}$ in the $\mathrm{SF}_{6}$ plasma and $1.31 \times 10^{-3} \mathrm{~Pa}$ in the $\mathrm{CF}_{4}$ plasma, as the bias voltage is increased from $-200 \mathrm{~V}$ to $-350 \mathrm{~V}$ and $-400 \mathrm{~V}$ respectively.

The ion current density $\left(\mathrm{I}_{i}\right)$, as a function of bias voltage is shown in figure 5.7(b). The results at $0 \mathrm{~V}$ bias again refer to the flow of the process gases whereby the ion current density in the reactor falls to zero. The ion current density is seen to be higher in the $\mathrm{SF}_{6}$ plasma at $200 \mathrm{~V}$, although at $-300 \mathrm{~V}$ and above the $\mathrm{CF}_{4}$ plasma produces a higher ion current density by approximately $0.01 \mathrm{~mA} / \mathrm{cm}^{2}$. The difference in $\mathrm{I}_{i}$ at higher voltages is small compared to the difference when the reactor was held at $200 \mathrm{~V}$, at which the $\mathrm{I}_{i}$ in $\mathrm{SF}_{6}$ was $0.03 \mathrm{~mA} / \mathrm{cm}^{2}$ greater than in the $\mathrm{CF}_{4}$ plasma. As the bias voltage increases, 


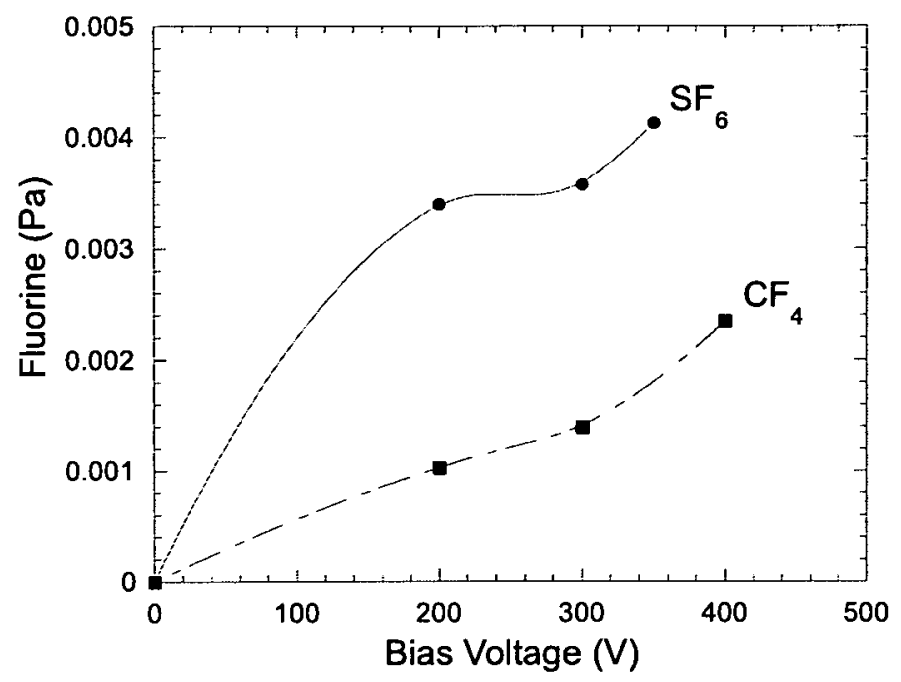

(a) The fluorine pressure as a function of bias voltage

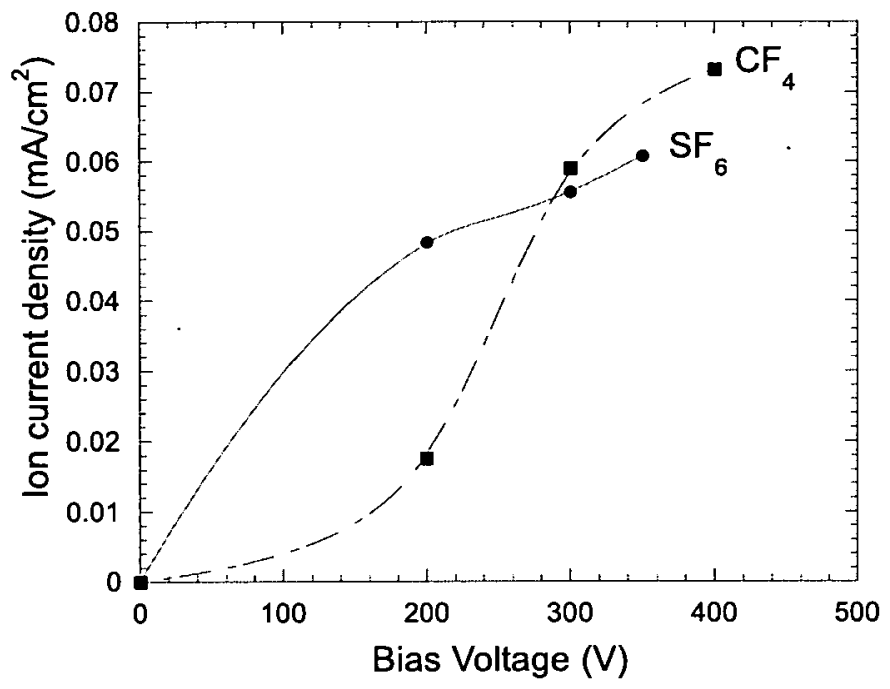

(b) The ion current density as a function of bias

Figure 5.7: The plasma conditions as measured by OES and LP plasma diagnostics. The fluorine pressure and ion current densities are shown as a function of bias voltage. The $\mathrm{CF}_{4}$ flow rate is $40 \mathrm{sccm}, S F_{6}$ flow rate at $20 \mathrm{sccm}$

the overall $\mathrm{I}_{i}$ increases for both plasma compositions, although the $\mathrm{SF}_{6}$ plasma shows less bias voltage dependence. 


\subsubsection{CNT Surface Characterisation}

The ratio of $\mathrm{F} / \mathrm{C}$ attached to the CNTs as a function of bias voltage is shown, figure 5.8(a), from the XPS data collected 4 weeks after the functionalisation procedure ${ }^{2}$. The $\mathrm{F} / \mathrm{C}$ ratio is higher in the $\mathrm{SF}_{6}$ exposed CNTs at low bias voltages although it appears to saturate at $300 \mathrm{~V}$, after which there is little increase in $\mathrm{I}_{i}$. The functionalisation process used here is sensitive to the reactor conditions, and there is a required balance between the amount of fluorine available and the ion current density to allow for successful attachment. The increase in the $\mathrm{F} / \mathrm{C}$ ratio seems to depend both on the ion current density increase and the increase in fluorine pressure. The $\mathrm{CF}_{4}$ exposed CNTs show the highest $\mathrm{F} / \mathrm{C}$ ratio of all at $400 \mathrm{~V}$ at which point $\mathrm{I}_{i}$ and F-pressure have both increased. However, the F-pressure is ultimately higher in the $\mathrm{SF}_{6}$ plasma leading to the conclusion that the $\mathrm{F} / \mathrm{C}$ ratio shows more of a dependence on $\mathrm{I}_{i}$.

The binding energy of the C-F bonds, going from semi-ionic to covalent in the F1s peak, is thought to depend primarily on the amount of fluorine available, where a higher fluorine pressure in the $\mathrm{SF}_{6}$ plasma has resulted in a more covalent $\mathrm{C}-\mathrm{F}$ bond over all reactor conditions. In Section 5.2.2 and previous work [17] it was observed that within a $\mathrm{CF}_{4}$ plasma, the intensity ratio of $\mathrm{I}_{\text {semi-ionic }} / \mathrm{I}_{\text {covalent }}$ in the $\mathrm{F} 1 \mathrm{~s}$ peak decreased with an increase in the $\mathrm{CF}_{4}$ gas flow. The F-pressure in the $\mathrm{CF}_{4}$ plasma never reaches the F-pressure achieved in the $\mathrm{SF}_{6}$ plasma, see figure 5.7, over all reactor conditions, thus always requiring a semi-ionic and covalent F1s peak component and never only a covalent bond component as is the case for the $\mathrm{SF}_{6}$ exposed CNTs.

The percentage of oxygen attached to the CNTs is also investigated by XPS, figure 5.8(b) and again the results taken 4 weeks after functionalisation are shown. The oxygen present here is due to contamination from the atmosphere. It is observed that the oxygen to carbon ratios are higher in the $\mathrm{CF}_{4}$ plasma exposed $\mathrm{CNT}$ and reach a saturation, similar to that observed for the $\mathrm{F} / \mathrm{C}$ in the $\mathrm{SF}_{6}$ plasma exposed CNTs. It is proposed that the oxygen is bonded to the CNTs via surface attachment at ion induced defect sites as the oxygen ratio increases with bias voltage. The tubes exposed at $0 \mathrm{~V}$ and the control sample display oxygen due to defect sites already present on the CNTs after manufacturing as well as to the bucky paper preparation process.

\footnotetext{
${ }^{2}$ The latter data is presented as it is believed that during the time interval elapsed at exposure to atmosphere any oxygen bonding to the CNTs at plasma induced defect sites will have taken place.
} 


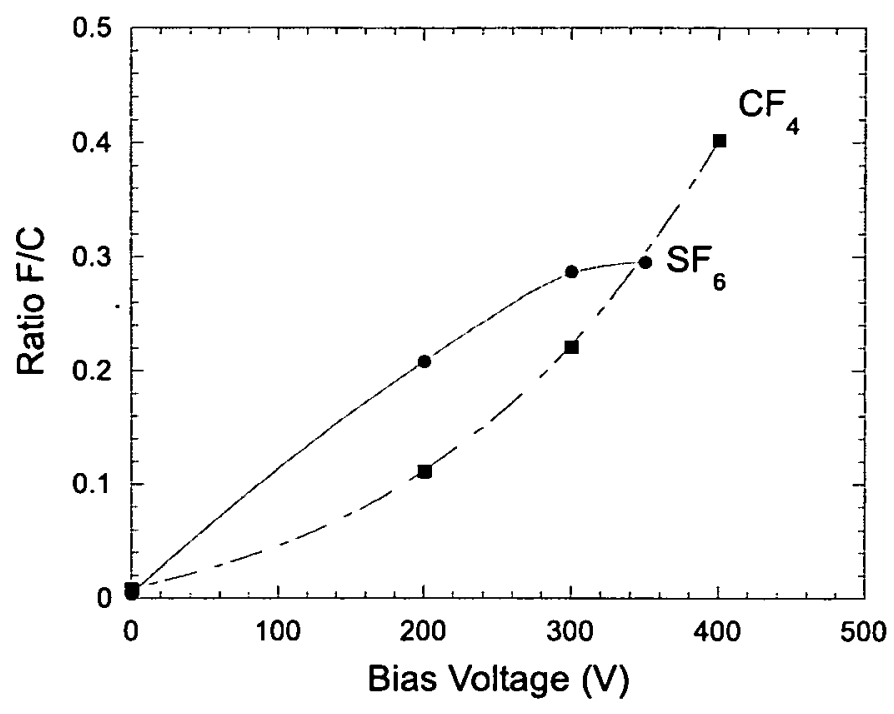

(a) The fluorine to carbon ratio as a function of bias

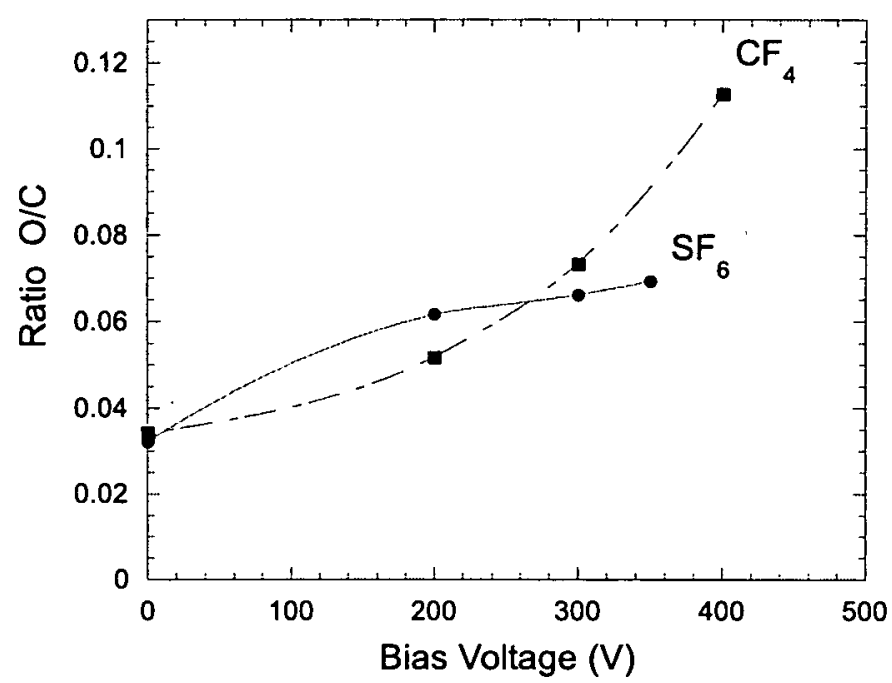

(b) The oxygen to carbon ratio

Figure 5.8: The surface characteristics of the CNT bucky papers as a function of bias voltage. The $\mathrm{CF}_{4}$ flow rate is $40 \mathrm{sccm}, \mathrm{SF}_{6}$ flow rate at $20 \mathrm{sccm}$

The F1s spectra have been observed to remain unchanged with time, with the binding energies constant and hence it is concluded that the fluorine is well attached to the nanotubes. It is interesting to note that for all CNTs exposed to a plasma environment, the atomic percentage of oxygen increased over time. It is likely that oxygen from the atmosphere has bonded to the CNTs via defect sites created by the ion bombardment during the plasma exposure. The $\mathrm{O} / \mathrm{C}$ ratio closely follows the trend observed for the ion current density, see figures 5.7(b) and 5.8(b). For example, the $\mathrm{O} / \mathrm{C}$ ratio is higher 
for the $\mathrm{CF}_{4}$ exposed tubes than the $\mathrm{SF}_{6}$ exposed at bias voltage conditions above -300 $\mathrm{V}$ DC bias, figure $5.8(\mathrm{~b})$ in agreement with the higher $\mathrm{I}_{i}$ present in the $\mathrm{CF}_{4}$ plasma, figure 5.7(b).

It is noted that there is no possibility of further F-attachment as no further fluorine exposure has taken place, and the atmosphere would be unable to produce such an effect. However, it may be the case that for the $\mathrm{SF}_{6}$ exposed CNTs, which were exposed to a higher $\mathrm{F}$ pressure throughout the scan, the fluorine attached to defect sites made by the plasma, limiting the remaining regions for atmospheric oxygen to attach.

\subsubsection{The Position of the F1s Peak}

The position of the F1s peak as discussed in the previous sections, is indicative of the covalencey of the bond between the carbon and the fluorine. The plasma feed stock gas drastically alters the position of the F1s peak, with a higher F content resulting in a move covalent C-F bond, shown in figure 5.9 .

It is noted that in the case of the $\mathrm{O}_{2}$ feed stock gas there is still an ionic F1s component present on the CNT surface, even though no fluorine feed stock gas was used. The percentage value of $\mathrm{F}$ on the surfaces in this case is very small. It is presumed that the reactor sidewalls were not completely clean and therefore it was still possible for small amounts of fluorine present in the reactor to have been sputtered from the reactor sidewalls and to attach to the CNT sidewalls. Due to the high electronegativity of fluorine even small percentage amounts in the plasma environment will easily attach to the CNTs.

The higher fluorine pressure in the $\mathrm{SF}_{6}$ plasma has led to a more covalent $\mathrm{F} 1 \mathrm{~s}$ peak than the $\mathrm{CF}_{4}$ plasma exposure. However, by using a conductive substrate the $\mathrm{F} 1 \mathrm{~s}$ peak shifts higher to an even more covalent region in the case of the $\mathrm{SF}_{6}$ plasma. Presumably this is due to the positive fluorine ions in the plasma being more attracted to the negatively biased CNT surface and having a higher acceleration through the plasma sheath region, when conductive substrates are used. Along with the ionic F1s peak in the oxygen plasma, the evidence points strongly towards covalent F1s occurring with higher fluorine pressure in the plasma. 


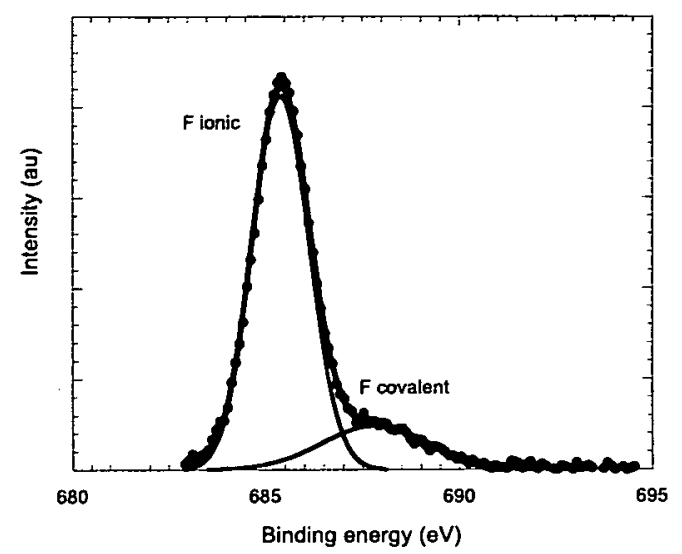

(a) The F1s peak of CNT on a PTFE substrate exposed to $\mathrm{O}_{2}$ plasma

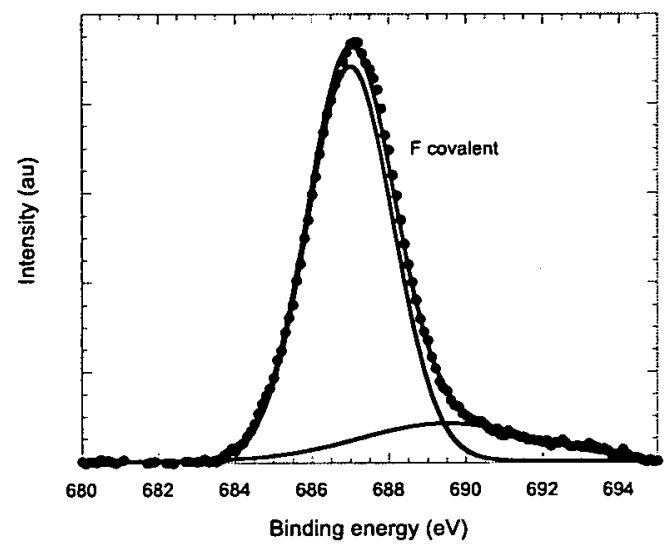

(c) The F1s peak of CNT on a PTFE substrate exposed to $\mathrm{SF}_{6}$ plasma

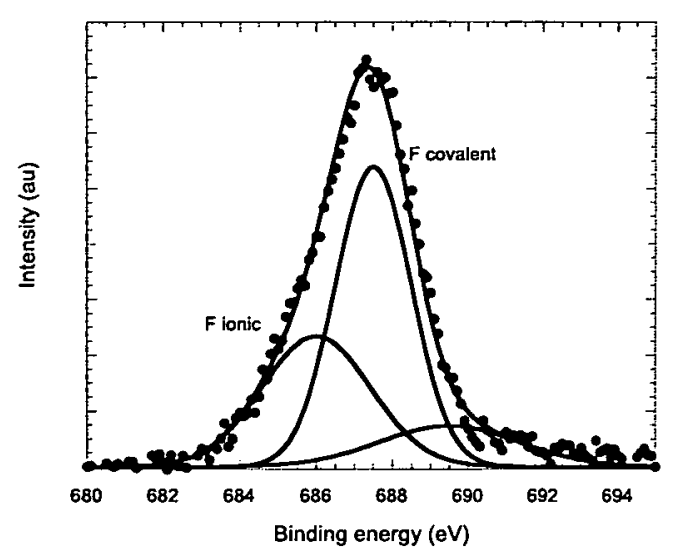

(b) The F1s peak of CNT on a PTFE substrate exposed to $\mathrm{CF}_{4}$ plasma

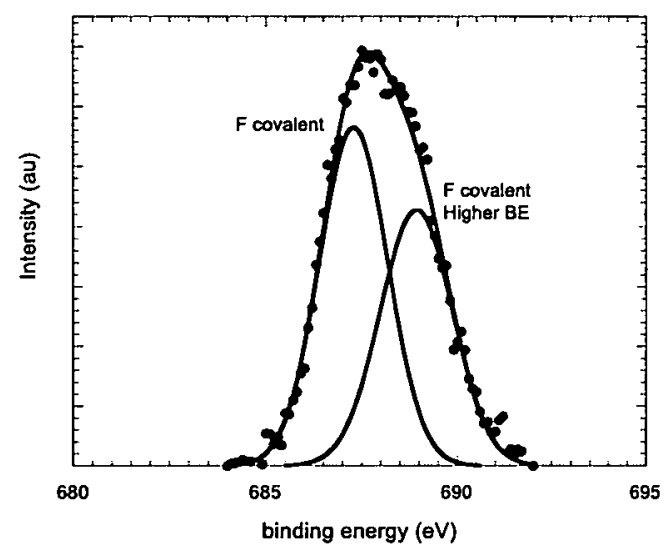

(d) The F1s peak of CNT on an aluminium substrate exposed to $\mathrm{SF}_{6}$ plasma

Figure 5.9: The F1s peaks for altered plasma feedstock gases. All samples were exposed to the plasma at -200 V bias for 1 minute. In the case of the $\mathrm{O}_{2}$ and $\mathrm{CF}_{4}$ plasma the flow was $40 \mathrm{sccm}$, whilst for the $S F_{6}$ plasma $20 \mathrm{sccm}$.

Other work by An et al. [118] had shown that the covalencey of the F1s region on fluorinated CNTs would increase with reaction temperature, which would also explain the covalent bonds found by Mickelson et al. when the reactions took place at elevated temperatures, ranging from $150^{\circ} \mathrm{C}$ to $600^{\circ} \mathrm{C}$ for several hours. As the process used for plasma fluorination exploited short functionalisation times, it has not been possible to draw a direct comparison between the covalencey over similar time periods. The increase in covalencey with fluorine content in the plasma feedstock is a desirable at- 


\begin{tabular}{c|c}
\hline \hline Feed stock gas & Main F1s component $(\mathrm{eV})$ \\
\hline $\mathrm{O}_{2}$ & 685 \\
$\mathrm{CF}_{4}$ & 686 \\
$\mathrm{SF}_{6}$ & 687 \\
$\mathrm{SF}_{6}^{*}$ & 688 \\
\hline
\end{tabular}

Table 5.3: Position of the F1s peak. *The CNTs are placed on a conducting substrate.

tribute for functionalisation control.

\subsubsection{Transmission Electron Microscopy of the CNTs Surface}

Although it is presumed that the ion bombardment within the plasma will weaken or produce defects in the CNTs structure, the $\mathrm{F} / \mathrm{C}$ ratios have not reached the saturation stoichiometry which would result in complete loss of tube structure. Transmission electron microscopy (TEM) images of the CNTs, before and after exposure to the plasma are shown in figures 5.10.
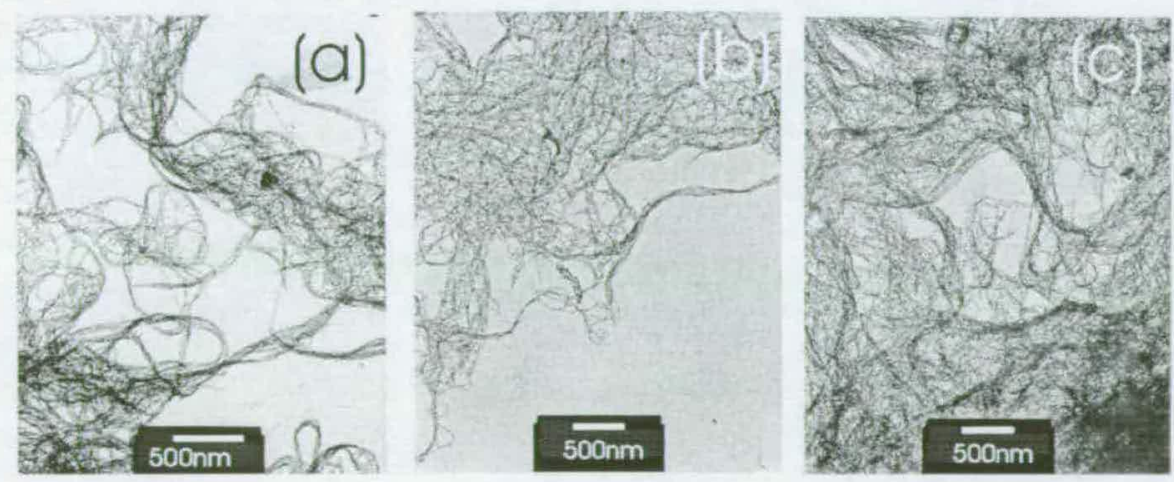

Figure 5.10: TEM images of a) pristine tubes, $b$ ) $C F_{4}$ plasma exposed samples and c) $S F_{6}$ plasma exposed samples

The TEM samples were prepared by making a weak suspension of CNTs, both pristine and functionalised, in IPA. A single drop of the CNT suspension was then drop coated onto $300 \mu \mathrm{m}^{2}$ carbon TEM grids from Agar. All three images shown are seen to be similar, with the CNTs forming bundles due to Van der Waals interactions, and the 
nanotubes appear to be intact. This has also been confirmed with Raman spectroscopy, whereby the signal showed strong radial breathing modes and a large G-band relative to D-band as was the case for the $\mathrm{CF}_{4}$ exposed CNTs shown in Section 5.2.2.

\subsubsection{Summary of $\mathrm{CF}_{4}$ and $\mathrm{SF}_{6}$ Plasma Exposed CNTs and the Attachment Mechanism}

In summary, the functionalisation of CNTs in a plasma environment has been carried out using both a $\mathrm{CF}_{4}$ and $\mathrm{SF}_{6}$ plasma. The actinometry and ion current density results have shown that in a plasma environment with a greater atomic fluorine pressure, a more covalent C-F attachment is observed after deconvolution of the F1s spectra, as was the case for the $\mathrm{SF}_{6}$ plasma in comparison to the $\mathrm{CF}_{4}$ plasma over all bias conditions. A bias voltage is required for fluorine to attach to the CNTs.

The $\mathrm{F} / \mathrm{C}$ ratio is influenced more by the $\mathrm{I}_{i}$ within the plasma rather than the F-pressure. The $\mathrm{O} / \mathrm{C}$ ratio is seen to depend on $\mathrm{I}_{i}$ and has increased over time. It is concluded that the ion bombardment within the plasma allows sufficient energy for the $\mathrm{C}$ - $\mathrm{C}$ bonds to be weakened or even broken, hence allowing for the attachment of fluorine in the plasma system. However, the ion bombardment has not resulted in loss of nanotube structure. When there has been insufficient fluorine to attach to all the weakened bonds or defect sites atmospheric oxygen has been able to attach to the CNTs.

The fluorine attachment is seen to remain stable over time, showing that the functionalisation process is a reliable means of fluorinating CNTs.

\subsection{Conclusion}

Exposing CNTs to a fluorinated plasma for short exposure times has repeatedly functionalised the CNTs without damaging the unique CNT structure. The effect of changing $\mathrm{CF}_{4}$ plasma exposure parameters and the resulting changes to the single-wall carbon nanotubes has been studied. Raman spectroscopy results indicate that the modifications to the CNTs structure have been subtle and that the structural integrity of the tubes has been maintained. The macroscopic appearance of the CNTs before and after plasma exposure, are shown to be similar from the SEM images. 
X-ray photoelectron spectroscopy has shown that fluorine can be attached to CNTs using a $\mathrm{CF}_{4}$ plasma. The intensity ratio of $\mathrm{I}_{\text {semi-ionic }} / \mathrm{I}_{\text {covalent }}$ of the $\mathrm{F} 1 \mathrm{~s}$ peak is seen to decrease upon the increase of flow rate of the plasma gas and exposure time of the samples to the $\mathrm{CF}_{4}$ plasma, showing that the fluorination process is sensitive to the reactor conditions. The low $\mathrm{F} / \mathrm{C}$ ratios of the CNTs confirm that the fluorination process has not yet reached saturation.

The functionalisation of CNTs in a plasma environment has been carried out using both a $\mathrm{CF}_{4}$ and $\mathrm{SF}_{6}$ plasma. The actinometry and ion current density results have shown that in a plasma environment with a greater atomic fluorine pressure, a more covalent $\mathrm{C}-\mathrm{F}$ attachment is observed after deconvolution of the F1s spectra, as was the case for the $\mathrm{SF}_{6}$ plasma in comparison to the $\mathrm{CF}_{4}$ plasma over all bias conditions. A bias voltage is required for fluorine to attach to the CNTs.

The $\mathrm{F} / \mathrm{C}$ ratio is influenced more by the $\mathrm{I}_{i}$ within the plasma rather than the F-pressure. The $\mathrm{O} / \mathrm{C}$ ratio is seen to depend on $\mathrm{I}_{i}$ and has increased over time. It is concluded that the ion bombardment within the plasma allows sufficient energy for the $\mathrm{C}$ - $\mathrm{C}$ bonds to be weakened or even broken, hence allowing for the attachment of fluorine in the plasma system. However, the ion bombardment has not resulted in loss of nanotube structure. When there has been insufficient fluorine to attach to all the weakened bonds or defect sites atmospheric oxygen has been able to attach to the CNTs.

The plasma functionalisation process has shown an advantage over previous methods, since the functionalisation time is considerably reduced from the several hours needed in other work $[8-10,118,145,158]$. Not only this, highly elevated temperatures are not needed here.

The fluorine attachment is seen to remain stable over time, showing that the plasma functionalisation process is a reliable means of fluorinating CNTs. In particular the plasma environment has allowed for the study of the mechanism of CNT functionalisation and has shown that the CNTs can withstand the somewhat aggressive nature of a plasma environment and maintain their structural integrity. The recent advent of other authors using similar methods to the plasma functionalisation process used here $[11,14,146]$ has shown the versatility of plasma fluorinated CNTs.

In the next chapter, the CNTs will be exposed to a $\mathrm{CF}_{4}$ plasma before being further 
functionalised with diamine. The aim of these experiments is to convert the inherently p-type field dependent behaviour of CNTs to n-type. Both the fluorine exposed and the diamine functionalised electronic properties will be discussed. 


\section{Chapter 6 Diamine Functionalisation of Plasma Fluorinated Carbon Nanotubes}

The fluorinated carbon nanotubes have been further functionalised with amine containing groups to change the CNTs from inherent p-type semiconductors to n-type. In the following chapter a description of the experimental methods used to produce n-type CNT devices is made.

Here we aim to exploit the advantages of the $\mathrm{CF}_{4}$ plasma functionalisation process, such as the short processing time and the maintaining of structural integrity of the CNTs, to then further functionalise the fluorinated CNTs (F-CNTs) into amine functionalised (NCNTs). The substitution of 1,2-diaminoethane has been employed, following a modified version of the reaction developed by Stevens et al. [16]. The electrical behaviour of CNTs exposed to a plasma environment and further amine functionalisation will then be discussed.

\subsection{N-type Doping of CNTs, a Brief Review}

The use of amine containing groups to alter the inherently p-type CNTs to n-type devices has been exploited by several groups due to the amine's electron donating nature. The use of polyethylene imine (PEI) first carried out by Dai et al. [15] provided permanently n-type doped CNT devices, this was further expanded to produce soluble n-type CNTs starting with $\mathrm{CNT}(\mathrm{COCl})_{n}$ functionalised tubes [94]. CNTs have been doped with amine groups by butylamine [16, 92], 3'-((aminopropyl)triethoxy silane) (APTES) [91, 92], proplyamine [92] and hydrazine [95] to alter the CNT electrical behaviour. Amine functionalisation methods have also included the exposure of CNTs to an amine based plasma [14]. In-situ doping during growth [159-161] and doping with $\mathrm{K}, \mathrm{Cs}, \mathrm{Br}_{2}$ and 
$\mathrm{I}_{2}[67,85,86,88-90]$ to produce both p-type and n-type behaving tubes respectively has been employed to alter CNT conduction. The electrical behaviour of CNT devices has also been shown to change upon annealing of the devices [67,85-87] and ambipolar devices can even be made by controlled doping over specific regions of the CNT [85].

The Stevens group had used F-CNTs which were subjected to long gas exposure times at $150^{\circ} \mathrm{C}$ as described by the Rice University group [158], to reach $\mathrm{C}_{2} \mathrm{~F}$ stoichiometry. During this experiment the reaction time during the amine functionalisation was lengthened in an attempt to remove all the fluorine from the CNTs and to saturate the CNTs with nitrogen dopant to create fully n-type behaving $\mathrm{CNTs} \mathrm{CF}_{4}$ plasma functionalisation of CNTs similar to the method developed here and presented in Chapter 5 , has also been carried out by Valentini et al. in 2005 [11]. The Valentini group then continued to further functionalise their fluorinated CNTs with butylamine [12]. Butylamine has only one amine group capable of doping the CNTs as opposed to 2 in the $\mathrm{N}$-alkyidene amine groups put forward by the Stevens group.

In this chapter the plasma fluorinated CNTs have been further reacted with 1,2- diaminoethane to convert p-type CNT devices to n-type devices. The amine acts as an electron donator in such a situation. The experimental procedures and the CNT characterisation is discussed in this chapter.

\subsection{Experimental Method of the F-tube Diamine Functionali- sation}

Single-walled carbon nanotubes, $\mathrm{HiPCO}$ from $\mathrm{CNI}$, were fluorinated in a $\mathrm{CF}_{4}$ plasma at $40 \mathrm{sccm}$ flow rate for periods of 10,20,40 and 60 seconds at the low bias end of the reactor; D.C. bias -30 V. The reaction with diamines developed by Stevens et al. [16] was employed to functionalise fluorinated CNTs with 1,2-diaminoethane. Since it was found by Stevens et al. that a reaction time of three hours was insufficient for full conversion to the amine substituent, the reaction time here was increased to 6 hours $^{1}$.

Plasma fluorinated CNTs were added to 1,2-diaminoethane $\left(5 \mathrm{~cm}^{3}\right)$ and sonicated briefly ( 5 mins) to aid dispersion. A catalytic quantity of pyridine ( 5 drops) was added

\footnotetext{
${ }^{1}$ The amine functionalisation reaction was carried out by Gavin Forrest a collaborator from the School of Chemistry at The University of Edinburgh. email: G.A.Forrest@sms.ed.ac.uk
} 
and the suspension was stirred under argon at reflux $\left(116^{\circ} \mathrm{C}\right)$ for 6 hours. The reaction vessel was allowed to cool to room temperature and filtered on a $0.2 \mu \mathrm{m}$ micropore filter to leave a black nanotube residue that was washed twice with ethanol.

1,2-diaminoethane was chosen in contrast to diamines with longer alkyl spacers such as alkylamines for two reasons. Firstly, the multiplicity of amine groups should increase the degree of electron donation to the nanotube. Secondly, by using shorter spacers electron transport through the molecule from the second amine group is facilitated. Reaction at both ends of the diamine is predicted to occur, either with fluorine on the same or different CNTs as shown in figure 6.1.
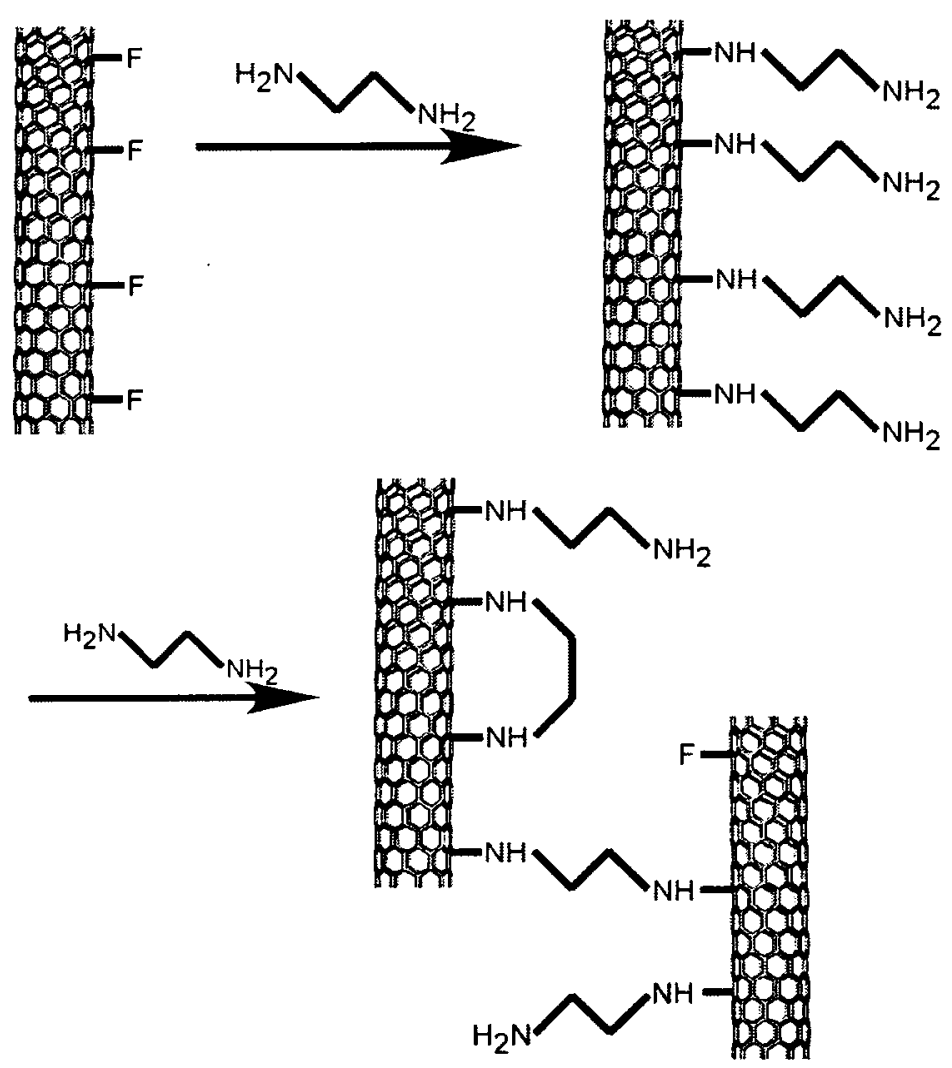

Figure 6.1: Schematic representation of the amine-functionalisation process. An individual fluorine atom can become substituted for 2 amine groups, or share the second amine with a nearby fluorine atom via a bi-functionalisation process.

X-ray photoelectron spectroscopy and Raman spectroscopy are both used to characterise the CNT functionalisation as discussed in Chapter 3 and in Chapter 5 . The $\mathrm{CF}_{4}$ plasma fluorinated CNTs will be referred to as F-CNTs and the diamine functionalised as NCNTs. 


\subsection{Functionalised CNTs Spectroscopy}

X-ray photoelectron spectroscopy (XPS) of the plasma fluorinated and the further functionalised amine exposed samples, have shown both functionalisation processes to be successful. There is a clear difference between the plasma fluorinated CNTs and the amine functionalised CNTs easily observable by the presence of fluorine and nitrogen in the XPS spectra respectively. The Raman spectra has also shown that the CNTs remain intact over all functionalisation conditions tested here.

The following sections discuss the XPS spectra and the Raman spectra. As the functionalisation results are coupled, the discussion will first show the stoichiometries achieved during the functionalisation. The specific regions of interest in the XPS spectra - the $\mathrm{C} 1 \mathrm{~s}, \mathrm{~F} 1 \mathrm{~s}$ and N1s regions will then be considered, followed by the structural inference and the disorder of the CNT $\mathrm{sp}^{2}$ bonding measured by Raman spectroscopy.

\subsubsection{Atomic Ratios on the CNT Surfaces}

The stoichiometries of the functionalised CNTs are shown in table 6.1 along with the $\mathrm{CF}_{4}$ plasma exposure conditions.

\begin{tabular}{ccc|cccc}
\hline \hline \multicolumn{2}{c|}{$\mathrm{CF}_{4}$ plasma conditions } & \multicolumn{4}{c}{ Stoichiometry } \\
$\mathrm{CF}_{4}$ exposure time & $\mathrm{DC}$ Bias & Gas flow & Fluorinated & $\mathrm{O} / \mathrm{C}$ & aminated & $\mathrm{O} / \mathrm{C}$ \\
\hline $10 \mathrm{~s}$ & $30 \mathrm{~V}$ & $40 \mathrm{sccm}$ & $\mathrm{C} \mathrm{F}_{0.300}$ & 0.222 & $\mathrm{C} \mathrm{N}_{0.065} \mathrm{~F}_{0.015}$ & 0.118 \\
$20 \mathrm{~s}$ & $30 \mathrm{~V}$ & $40 \mathrm{sccm}$ & $\mathrm{CF}_{0.514}$ & 0.262 & $\mathrm{C} \mathrm{N}_{0.068} \mathrm{~F}_{0.014}$ & 0.108 \\
$40 \mathrm{~s}$ & $30 \mathrm{~V}$ & $40 \mathrm{sccm}$ & $\mathrm{CF}_{0.633}$ & 0.104 & $\mathrm{C} \mathrm{N}_{0.077} \mathrm{~F}_{0.019}$ & 0.103 \\
$60 \mathrm{~s}$ & $30 \mathrm{~V}$ & $40 \mathrm{sccm}$ & $\mathrm{C} \mathrm{F}_{0.546}$ & 0.130 & $\mathrm{C} \mathrm{N}_{0.059} \mathrm{~F}_{0.013}$ & 0.089 \\
\hline
\end{tabular}

Table 6.1: The plasma exposure conditions and the resulting stoichiometry of the CNTs post functionalisation

The atomic ratios on the CNT surface have been plotted against plasma exposure time figure 6.2. The degree of fluorination post $\mathrm{CF}_{4}$ plasma exposure can, in general, be seen to increase with plasma exposure duration. The anomalous 60 second sample 
can be attributed to the non linearity of the plasma process due to the number of variables involved, an effect also seen by other groups using $\mathrm{CF}_{4}$ plasma to functionalise CNTs [13].

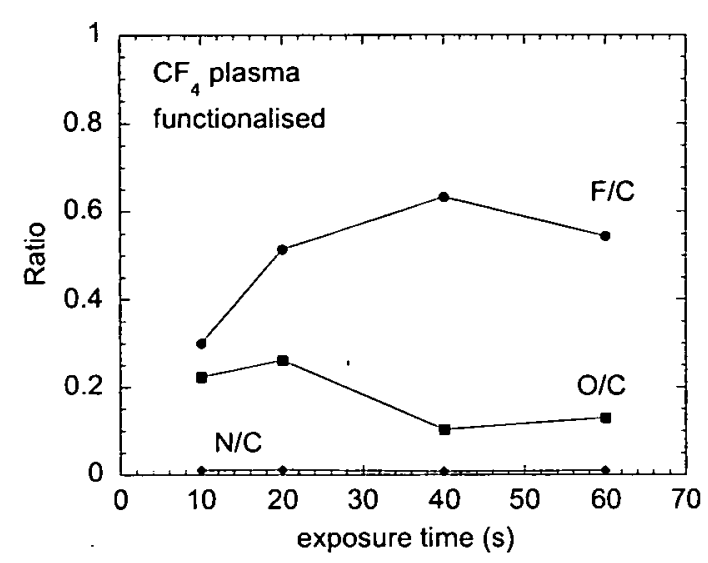

(a) The $\mathrm{F} / \mathrm{C}, \mathrm{O} / \mathrm{C}$ and $\mathrm{N} / \mathrm{C}$ ratios of the F-CNTs

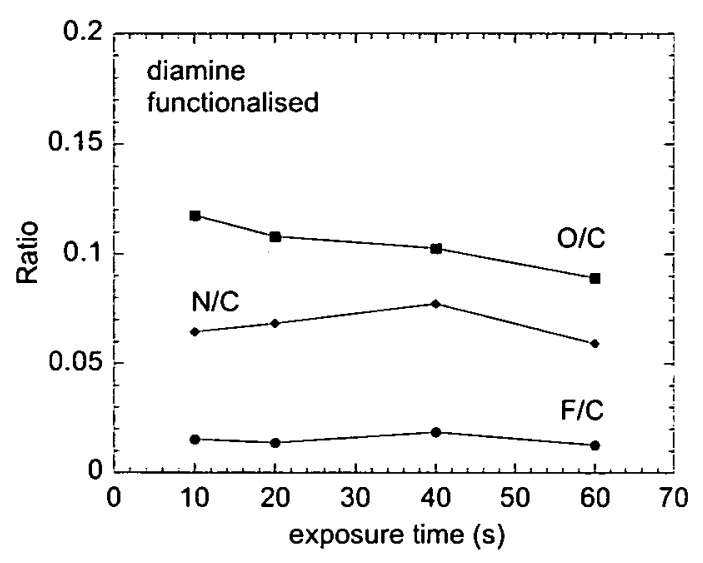

(b) The F/C, O/C and N/C ratios of the N-CNTs

Figure 6.2: The surface characteristics of the functionalised CNTs as a function of plasma exposure time

After diamine functionalisation the $\mathrm{F} / \mathrm{C}$ ratio of all the $\mathrm{CNT}$ batches reduced to approximately 0.015 regardless of the initial $\mathrm{F} / \mathrm{C}$ and the initial comparison between ionic and covalent fluorine. The N/C ratio was approximately constant throughout the samples, with a slight increase at 40s plasma exposure, similar to the trend observed for the $\mathrm{F} / \mathrm{C}$ ratio of the plasma exposed tubes figure 6.2. The $\mathrm{O} / \mathrm{C}$ ratio decreases after diamine functionalisation although becomes the highest atomic ratio contributor to the functionalisation after the amine functionalisation process, steadily decreasing with plasma exposure time.

As discussed previously $[17,18]$ and in Chapter 5 , the plasma process exposes the CNTs to ion bombardment. The flux of ions reaching the CNT sample surface should not vary considerably over time, after steadying out in the first few seconds of bias voltage application, since the movement of electrons and ions is fast an equilibrium is soon reached. Given the ion bombardment and the creation of defects in the CNT sidewalls such bombardment would create, the functionalisation via fluorination will become more efficient over time as there is a trade off between defect creation and available radical concentration capable of bonding to the defect sites on the CNTs. It appears as though not all defect sites were fluorinated from the oxygen concentration visible. However, 
the similarity in trend between the $\mathrm{F} / \mathrm{C}$ ratio and the $\mathrm{N} / \mathrm{C}$ ratio and the constant $\mathrm{F} / \mathrm{C}$ ratio over all conditions on the N-CNTs, shown in figure 6.2 implies that almost complete saturation of the amine functionalisation was achieved.

The most striking observation from table 6.1 and figure 6.2 is that the degree of functionalisation in the N-CNTs is considerably less than the F-CNTs, an effect that is also observed by both Stevens et al. [16] and Valentini et al. [11,12] raising the question of what happens to the majority of the fluorine during the amine functionalisation reaction?

The simplest explanation may be that some of the fluorine is only adsorbed onto the nanotubes rather than being chemically attached. Within the $\mathrm{CF}_{4}$ plasma we presume the creation of radicals and molecular $\mathrm{CF}_{2}$ and $\mathrm{F}_{2}$, as well as charged ions, such as $\mathrm{C}_{x} \mathrm{~F}_{y}^{+}$and $\mathrm{F}^{+}$[155-157], that could all be contributing to the extra fluorine observed. At the low bias end of the reactor the acceleration between the plasma and the substrate due to the sheath region will be far lower than the high bias end. Such a lowering in acceleration could limit the covalencey of the C-F bonds, resulting in more adsorbed fluorine than the stoichiometries account for.

The C1s, F1s and N1s regions are now discussed as a function of plasma exposure time to determine the nature of the functionalisation process.

\subsubsection{The C1s Region}

Looking to the carbon C1s region in the XPS spectra of both the F-CNTs and the NCNTs, a clear difference can be seen between the two spectra, figure 6.3. The F-CNTs exhibit three peaks at $289 \mathrm{eV}, 291 \mathrm{eV}$ and $293 \mathrm{eV}$ which can be attributed to carbon 1s electrons in $\mathrm{CF}, \mathrm{CF}_{2}$ and $\mathrm{CF}_{3}$ respectively [9] or $\mathrm{C}-\mathrm{O}, \mathrm{C}=\mathrm{O}$ and $\mathrm{O}-\mathrm{C}=\mathrm{O}[118,121]$, figure 6.3(a). The N-CNTs, exhibit a broadening at $286 \mathrm{eV}$ due to the presence of diamine carbon atoms [114] and no high energy carbon peaks. Both functionalisation processes have resulted in oxygen residing on the CNT surface as confirmed by the XPS stoichiometries. However as the the high energy C1s peaks are not evident in the amine functionalised CNTs, it can be deduced that carbon fluorine bonds are responsible for the higher binding energy peaks observed for the F-CNTs samples.

As altering the plasma exposure times for the CNTs did not alter the positioning of the 


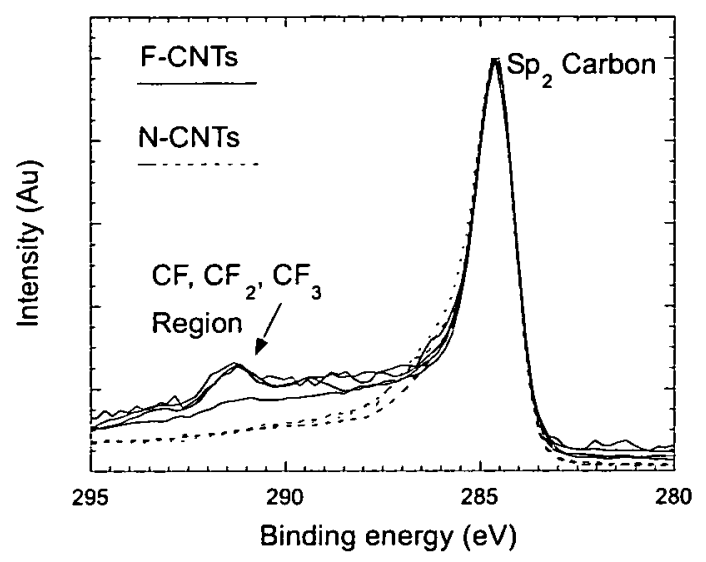

(a) The C1s peaks for the fluorinated tubes (solid line) and the aminated tubes (dashed line)

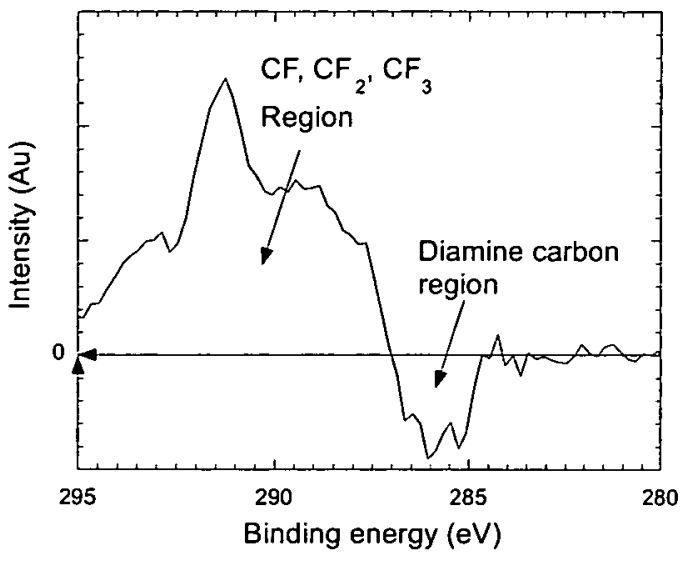

(b) An average of the XPS spectra of the F-CNTs minus the average XPS spectra for the N-CNTs

Figure 6.3: The normalised C1s spectra of the F-CNTs and the N-CNTs. The plasma exposure times were varied but caused no change in peak positions. All $\mathrm{CF}_{4}$ plasma exposures were carried out at $40 \mathrm{sccm}$ and D.C. bias $-30 \mathrm{~V}$.

C1s peaks, an average of the samples could be used to visualise the differences in the $\mathrm{C} 1 \mathrm{~s}$ region between the F-CNTs and the N-CNTs. By looking at the average normalised spectra of the F-CNTs minus the N-CNTs, figure 6.3(b), the change in the C1s spectra after the functionalisation steps is highlighted. The $\mathrm{C}_{x} \mathrm{~F}_{y}$ peaks are seen in the region from $290 \mathrm{eV}$ to $295 \mathrm{eV}$, whilst around $286 \mathrm{eV}$ a negative intensity at the diamine region is clearly observable. As such it is reasonable to assume the high energy $\mathrm{Cls}$ peaks are due to carbon fluorine bonds whilst the amine peak comes directly from the diamine functionalisation.

\subsubsection{The F1s Region}

To begin to characterise the functionalisation mechanism the F1s region of the initial plasma fluorinated CNTs is considered. The fluorine region of the XPS spectra showed two peaks, attributed to semi-ionic bonded fluorine and covalent bonded fluorine figure 6.4(a). For the F-CNTs the fluorine F1s peaks observed were similar to those seen in Chapter 5.

However, at the plasma exposure conditions used here, at the low bias end of the reactor, CNTs with higher F/C ratios (i.e. subjected to the plasma for longer, in general), exhibited C-F bonds with an increased semi-ionic/covalent ratio, figure 6.4(b). Such a 


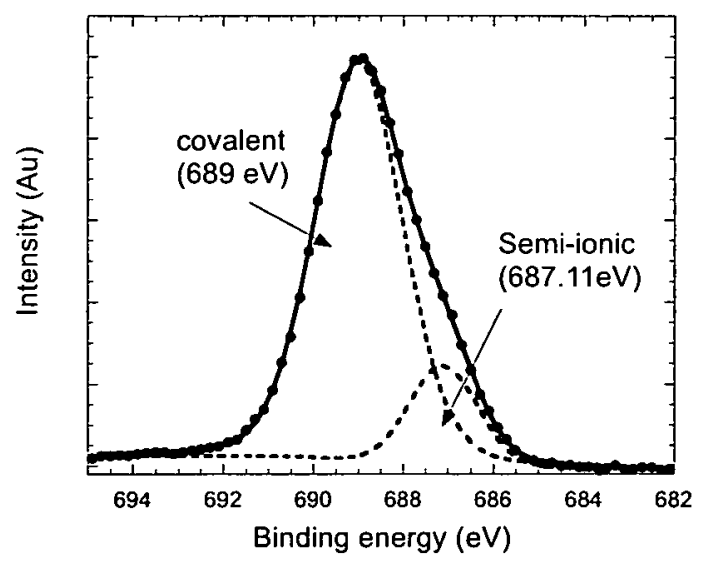

(a) The deconvoluted F1s spectra for the sample exposed to the $\mathrm{CF}_{4}$ plasma for $10 \mathrm{~s}$

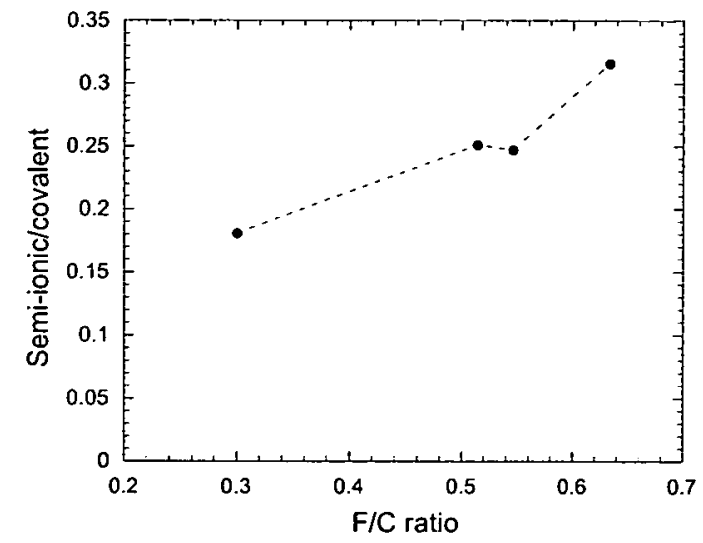

(b) The ratio of the semi-ionic to covalent bonding as a function of $\mathrm{F} / \mathrm{C}$ ratio

Figure 6.4: A typical F1s spectra of the functionalised CNTs. All $C_{4}$ plasma exposures were carried out at $40 \mathrm{sccm}$ and D.C. bias $-30 \mathrm{~V}$.

result is in contrast to previous discussion at the high bias end of the reactor, see [17] and Section 5.2.5, where increased exposure time led to decreased semi-ionic/covalent ratio. Such a decrease in the relative covalent bonding can be attributed to the reduced acceleration between the plasma and the biased substrate through the sheath region at low bias voltages. The directionality of the ion bombardment will be reduced at low bias and the loss of acceleration through the sheath may be allowing for a larger ratio of semi-ionic bonding to take place.

\subsubsection{The N1s Region}

When considering the further amine functionalisation the N1s region is studied. The nitrogen XPS region deconvoluted to two peaks, at $\approx 399.5 \mathrm{eV}$ and $\approx 400.5 \mathrm{eV}$ which can be attributed to nitrogen 1 s electrons in $\mathrm{NH}_{2}$ (primary amines) and $\mathrm{NH}$ (secondary amines) respectively [114]. Recalling the reaction schematic presented in figure 6.1, the secondary amines are a product of the reaction of 1,2-diaminoethane with the fluorinated nanotubes whilst the primary amines are thought to arise from unreacted amine groups. Due to the short length of the 1,2-diaminoethane molecule $(5 \AA)$ the degree of secondary reaction will be dependent on the availability of nearby C-F bonds. Therefore, it is likely that any bi-functionalisation will occur primarily with fluorines on the same nanotube, figure 6.1. 


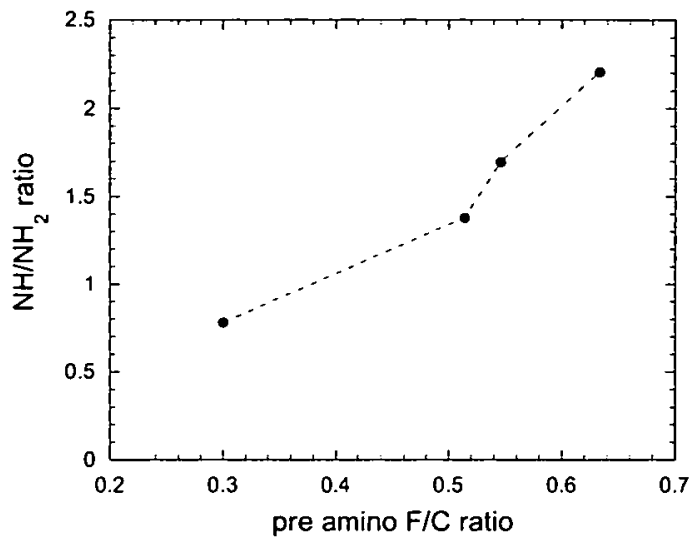

(a) The ratio of primary to secondary amines as a function of initial $\mathrm{F} / \mathrm{C}$ ratio

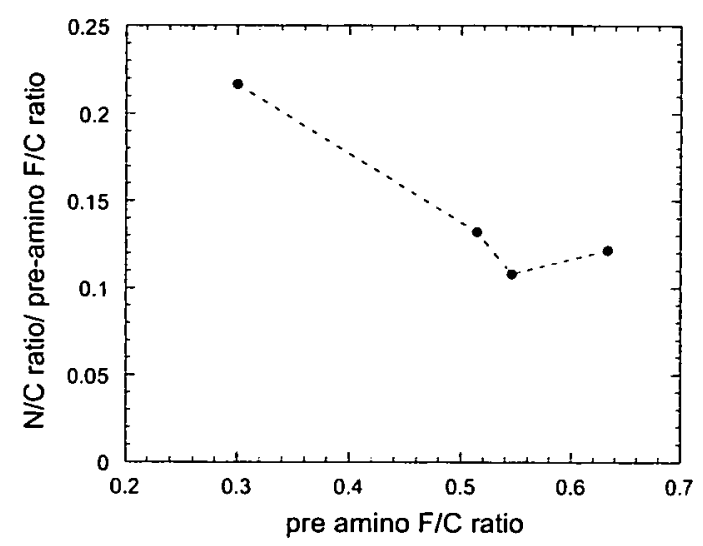

(b) Ratio of post amine functionalisation $\mathrm{N} / \mathrm{C}$ ratio, to initial $\mathrm{F} / \mathrm{C}$ ratio as a function of initial $\mathrm{F} / \mathrm{C}$ ratio

Figure 6.5: The diamine functionalisation as a function of initial $F / C$ ratio. (a) With increasing $F$ content, bi-functionalisation increases and more $\mathrm{NH}$ is present relative to $\mathrm{NH}_{2}$. (b) With increasing $\mathrm{F}$ content, the total relative $\mathrm{N}$ content decreases due to increased bi-functionalisation.

Figure 6.5(a) confirms the expected increase in bi-functionalised 1,2-diaminoethane with increasing fluorine content. Presumably such an increase is due to the increased availability of nearby fluorines making bi-functionalisation more favourable and more likely. Consequently, since carbon-bonded fluorine atoms are being replaced by 1,2diaminoethane molecules in a ratio less than $1: 1$ by an increased degree of bi- functionalisation, some decrease in the overall nitrogen content should be seen as a function of increasing pre-amine functionalisation fluorine content. This is indeed observed 6.5(b), where the total relative $\mathrm{N}$ content decreases with an increase in initial $\mathrm{F}$ content.

\subsubsection{The Raman Spectra}

The adsorption of fluorine, as opposed to bonding on the CNT surface, in the functionalisation processes used by other groups $[10,158]$ may also be the cause of their apparent incomplete removal of fluorine and failure to transfer all fluorine dopant to nitrogen. Here, Raman analysis supports this explanation. If all the fluorine detected by XPS were covalently attached to the CNTs the Raman spectra would show strong D-bands and a reduction in the RBM, which is not observed in figure 6.6.

In particular such an effect would be evident at $40 \mathrm{~s}$ plasma exposure, where there is clearly an apparent break of the $\mathrm{C}_{2} \mathrm{~F}$ limiting stoichiometry, known to cause structural 


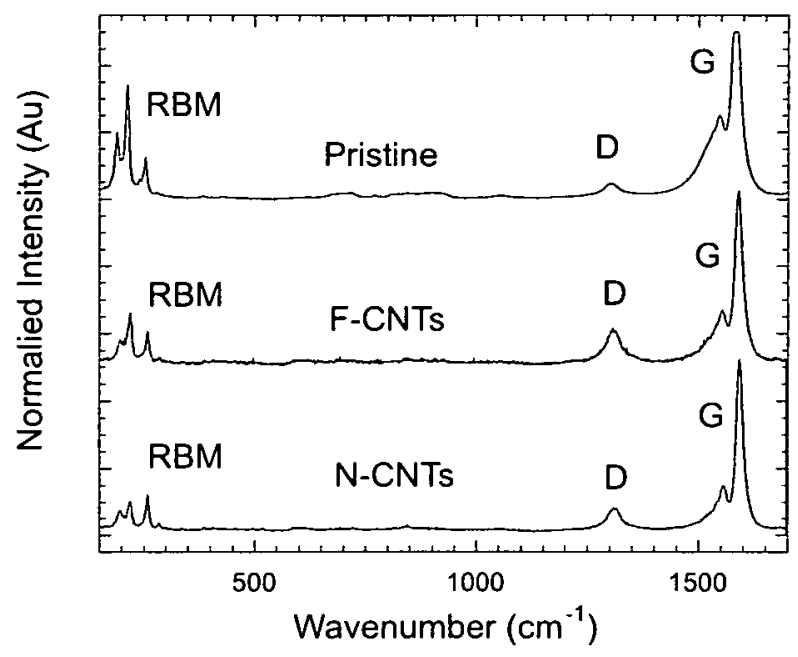

Figure 6.6: The typical Raman spectra from the pristine and functionalised CNTs.

damage to the CNTs [10]. As it is, strong radial breathing modes are observed in all samples along with a high G-band and low D-band (D/G ratios of typically 0.3 ), implying the CNTs are structurally intact [32].

It is of interest that the D-bands are slightly smaller after reaction with 1,2-diamino ethane, suggesting that some of the fluorine may have been removed without being replaced. The adsorption of $\mathrm{C}_{x} \mathrm{~F}_{y}^{+}, x$ and $y \geq 1$, in the $\mathrm{CF}_{4}$ plasma exposure stages could be a large contributor to such an effect.

\subsection{The Diamine Functionalisation Mechanism}

In the XPS spectra the $\mathrm{F} 1 \mathrm{~s}$ region has shown that $\mathrm{CF}_{4}$ plasma fluorination results in a combination of semi-ionic and covalent fluorine attached to the CNTs. After the amine functionalisation reaction both semi-ionic and covalent fluorine peaks were present on both the $10 \mathrm{~s}$ and $20 \mathrm{~s}$, plasma exposed samples however, the N-CNTs also show a lower semi-ionic/covalent fluorine ratio than the F-CNTs. The $40 \mathrm{~s}$ and $60 \mathrm{~s}$ plasma exposed samples both show only covalent fluorine post amine functionalisation.

At first glance this would suggest semi-ionic fluorine is preferentially substituted by 1,2 diaminoethane a reasonable assumption since the semi-ionic C-F bond is more charge separated. However, due to the unknown contribution by adsorbed fluorine, it is not possible to accurately determine the semi-ionic/covalent ratio of chemically bonded 
fluorine on the F-CNTs. Therefore, the F-CNT and N-CNT semi-ionic/covalent fluorine ratios cannot be compared and the observed "reduction" in the ratio may only be apparent.

It is expected that the reasonably severe conditions of the diamine functionalisation reaction will result in the removal of adsorbed fluorine and perhaps some weakly bonded fluorine, confirmed by the reduction of the D-band in the Raman spectra after amine functionalisation. Further work is required to determine the reaction mechanism, primarily the use of a $\mathrm{SF}_{6}$ plasma in comparison to the $\mathrm{CF}_{4}$ plasma, since a $\mathrm{SF}_{6}$ plasma is known to result in covalent $\mathrm{F}$ 1s peaks on the CNT surfaces ${ }^{2}$.

\subsubsection{Summary of the Diamine Functionalisation}

In summary it has been shown that substitution reactions of 1,2-diaminoethane onto plasma fluorinated CNTs has been successful. The reaction is limited by the initial fluorination of the CNTs themselves and it is shown that the ratio of nitrogen observed on the CNTs increases following the trend for the initial fluorination of each sample.

The ratio of the primary amines to secondary amines has been shown to increase with initial fluorine content implying that bi-functionalisation has taken place. The overall nitrogen content as a function of initial fluorine content was seen to decrease confirming that the replacement of fluorine with 1,2-diaminoethane molecules took place at a ratio of less than $1: 1$.

The exact mechanism of the functionalisation process has not been deduced, although the evidence points to a quantity of adsorbed fluorine increasing the initial F/C ratio. Functionalisation with a $\mathrm{SF}_{6}$ plasma should improve the amine functionalisation capabilities by reducing absorption and resulting in covalent fluorination.

\footnotetext{
${ }^{2}$ Further experiments are being carried out at the time of writing. Initial results have indicated that a sonication stage post $\mathrm{CF}_{4}$ plasma exposure, as done in Chapter 5 prior to XPS characterisation, removes adsorbed fluorine.
} 


\subsection{Electronic Characterisation of F-CNTs and N-CNTs}

\subsubsection{The Experimental Method}

Both the F-CNTs and the N-CNTs were separately dispersed in 1,2-dichloroethane before being spin coated onto electrode structures. The electrode setup is shown schematically in figure 6.7. The backgated silicon substrate was covered with $50 \mathrm{~nm}$ thermal $\mathrm{SiO}_{2}$ where $\mathrm{Ti} / \mathrm{Au} 5 / 25 \mathrm{~nm}$ source drain electrodes were fabricated on top by e-beam lithography. The electrode spacing was $500 \mathrm{~nm}$ for all device characteristics probed here. The CNTs were dispersed at weak concentrations, of $1 \mathrm{mg}$ CNTs in $10 \mathrm{ml}$ of 1,2-dichloroethane, as an attempt to achieve smaller bundles of CNTs bridging the electrodes, and one drop was coated on each sample. However large bundles of tubes still remained. The I-V characteristics were probed using an HP4156B probe analyser, as discussed in Chapter 3.3.

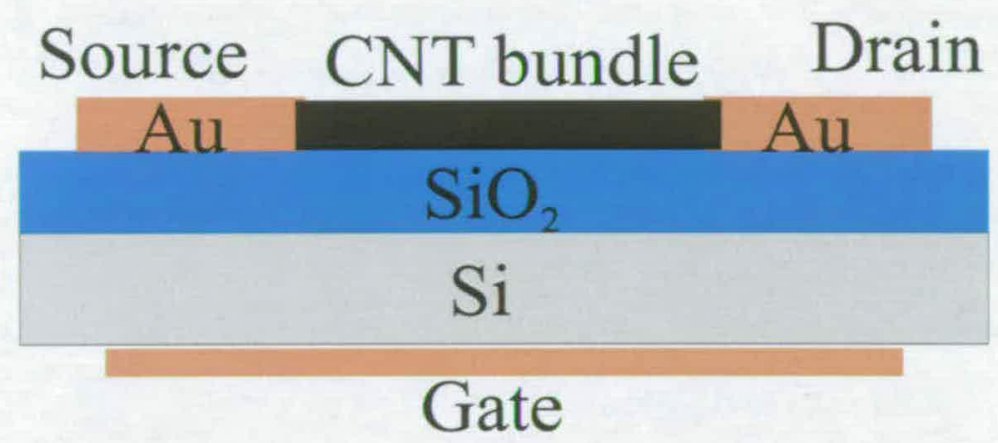

Figure 6.7: Schematic representation of the device setup

As is often discussed in the experimental considerations of carbon nanotube electronics the unreliability of the suspensions limits the reproducibility of the results [77]. When predicting the semiconducting or metallic behaviour of the CNTs due to statistical differences in the suspension mixture the observance of both metallic and semiconducting tubes is likely. Figure 6.8 shows an example of two separate bundles of CNTs bridging electrodes. There are clear differences in bundle size and therefore the chiralities and behaviour of each bundle can be drastically altered. Metallic conduction is more often observed in the average device properties where large bundles of CNTs are observed, and is presumed to be due to the dominance of the metallic conduction, in comparison to weak semiconducting effects. 


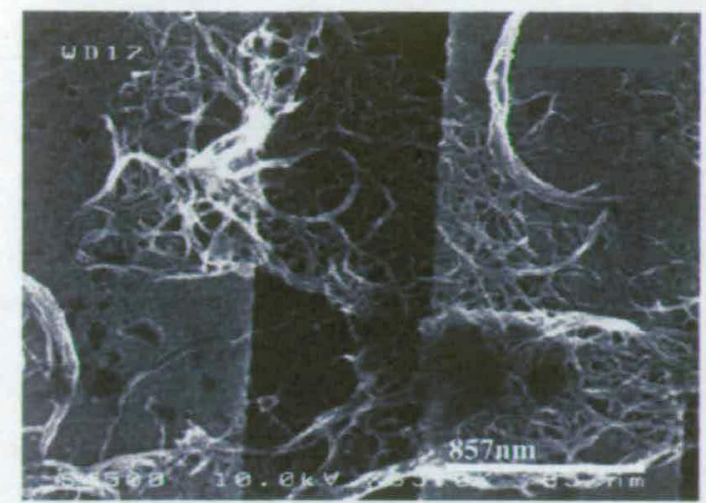

(a) A random bundle of CNTs bridging electrodes

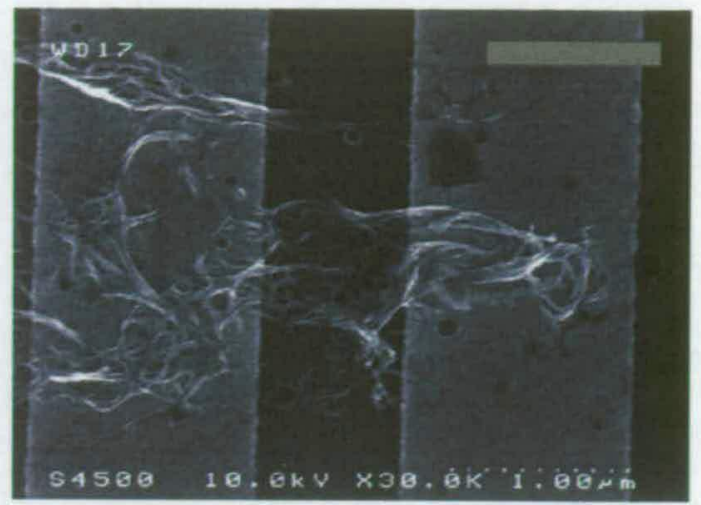

(b) A random bundle of CNTs bridging electrodes

Figure 6.8: The SEM images of randomly distributed functionalised HiPCO CNTs between electrodes. Image (a) and (b) show visibly different bundling properties and apparent differences in the number of CNTs. It is not possible by SEM to confirm the chirality distribution of the CNTs.

\subsubsection{Electronic Burn Off Techniques}

A technique known as "burn-off" is often applied to the devices $[60,77]$ to remove the metallic CNTs. "Burn-off" involves the application of a high potential to the CNT device. As metallic tubes will always conduct and semiconducting tubes will only conduct at specific gate voltages, the technique requires that the semiconducting tubes be turned off and the metallic tubes subjected to a high voltage. The heating effect of the resultant high current then breaks the CNTs by a simple burning process. By "burning-off" the metallic CNTs only semiconducting tubes should remain and field dependent electronic properties can then be studied. However, it should also be considered that in tight bundles of CNTs the burn-off will apply to semiconducting tubes in close vicinity of the metallic tubes.

The experimental characterisation here required the testing of the CNT devices by similar procedure, following the steps discussed using the probe setup and HP4156B as discussed in Chapter 3.3.4. A source drain voltage was applied from $-5 \mathrm{~V}$ to $+5 \mathrm{~V}$ at a spacing of $100 \mathrm{mV}$, the gate voltage as the second variable was altered from $\pm 5 \mathrm{~V}$ to $\mp 5 \mathrm{~V}$ at a spacing of $1 \mathrm{~V}$, the sign of the gate voltage depending on whether p-type or n-type semiconducting devices were to be turned off. The variable order was then altered with the gate voltage swept at $100 \mathrm{mV}$ spacing from $-5 \mathrm{~V}$ to $+5 \mathrm{~V}$ and the source drain voltage from $-2.5 \mathrm{~V}$ to $+2.5 \mathrm{~V}$ with $500 \mathrm{mV}$ spacing. Where the devices showed 


\begin{tabular}{|c|c|c|c|c|c|}
\hline \multicolumn{3}{|c|}{ Vds sweep. Vg held constant } & \multicolumn{3}{|c|}{ Vg step range } \\
\hline Start Vds & End Vds & Step & Start Vg & End Vg & Step \\
\hline$-5 \mathrm{~V}$ & $+5 \mathrm{~V}$ & $100 \mathrm{mV}$ & $\pm 5 \mathrm{~V}$ & $\mp 5 \mathrm{~V}$ & $1 \mathrm{~V}$ \\
\hline$-7.5 \mathrm{~V}$ & $+7.5 \mathrm{~V}$ & $100 \mathrm{mV}$ & $\pm-5 \mathrm{~V}$ & $\mp 5 \mathrm{~V}$ & $1 \mathrm{~V}$ \\
\hline \multicolumn{6}{|c|}{ Vds increased until burn-off or device breakdown } \\
\hline \multicolumn{3}{|c|}{ Vg sweep. Vds held constant } & \multicolumn{3}{|c|}{ Vds step range } \\
\hline Start Vg & End Vg & Step & Start Vg & End Vg & Step \\
\hline$-5 \mathrm{~V}$ & $+5 \mathrm{~V}$ & $100 \mathrm{mV}$ & $-2.5 \mathrm{~V}$ & $+2.5 \mathrm{~V}$ & $500 \mathrm{mV}$ \\
\hline$-10 \mathrm{~V}$ & $+10 \mathrm{~V}$ & $100 \mathrm{mV}$ & $-2.5 \mathrm{~V}$ & $+2.5 \mathrm{~V}$ & $500 \mathrm{mV}$ \\
\hline
\end{tabular}

Table 6.2: Standard probe station sweep conditions for backgated CNT devices

no gate voltage dependence, and therefore metallic behaviour, the source-drain voltage was then re-swept, and then increased to $-7.5 \mathrm{~V}$ to $7.5 \mathrm{~V}$ and further until burn-off occurred or in some cases complete breakdown of the devices. The sweep conditions listed above are shown in table 6.2.

\subsubsection{Source-Drain Electronic Behaviour of F-CNTs}

Shown in figure 6.9 are the source-drain characteristics for the CNT devices made post $\mathrm{CF}_{4}$ plasma functionalisation.

Current has flowed through all devices in a source drain sweep with $\mathrm{Vg}$ held at $0 \mathrm{~V}$. At $40 \mathrm{~s}$ and $60 \mathrm{~s}$ plasma exposure the drain current has increased by several orders of magnitude. The increase in conduction could be attributed to the CNT bundle itself, e.g. there are physically many more CNTs on this sample causing an increased current density. It is not possible from the magnitude of the drain current to determine the chirality of the CNTs present on any given device, however some information on the CNT behaviour can be deduced from the shape of the source-drain curves.

The shape of the source-drain curves has altered between the devices, with no two 


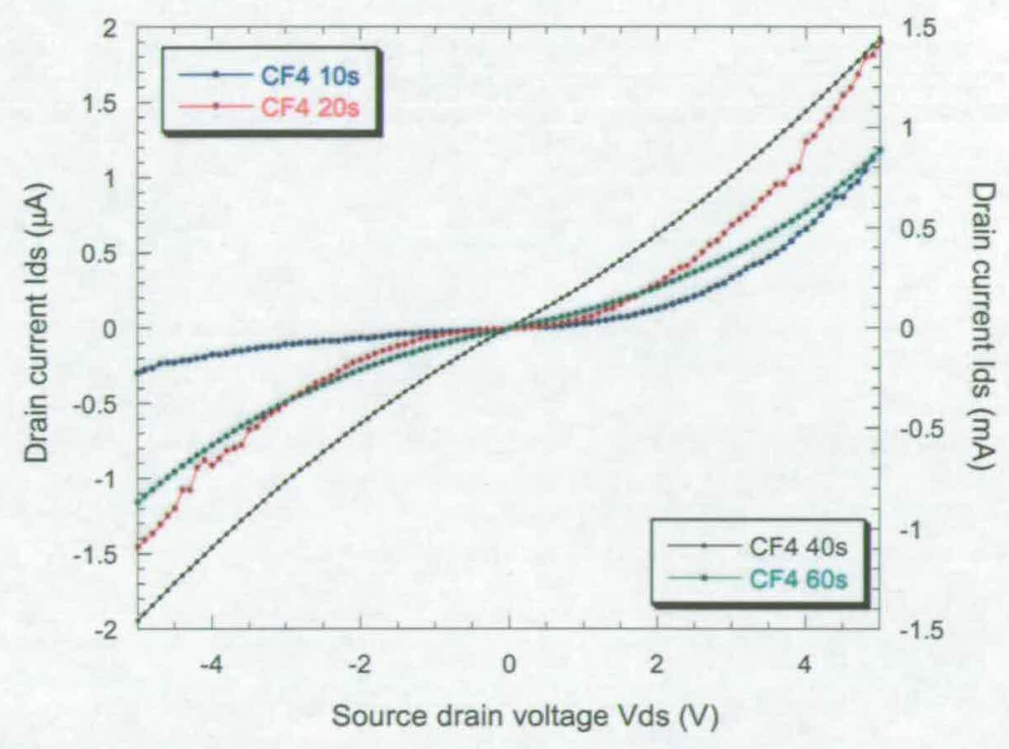

Figure 6.9: The source-drain characteristics for all F-CNTs devices at $V g=0 \mathrm{~V}$. The $40 \mathrm{~s}$ and $60 \mathrm{~s}$ plasma exposed sample uses the right hand side $y$-axis, the $10 \mathrm{~s}$ and 20 s the left.

devices showing identical source-drain behaviour. The $10 \mathrm{~s}$ plasma exposed F-CNTs device shows a highly asymmetrical curve indicative of back to back Schottky diodes. The $20 \mathrm{~s}$ and $60 \mathrm{~s}$ plasma exposed F-CNTs devices also show back to back Schottky diode character. The $40 \mathrm{~s}$ plasma exposed F-CNTs device in contrast shows a straight symmetrical line cutting through the origin, indicative of a metallic conductor. The $40 \mathrm{~s}$ plasma exposed sample had surpassed the structural breakdown stoichiometry as observed in table 6.1.

However, to determine the success of the functionalisation process for electronic applications, the CNT devices have been measured in backgated geometry to characterise their field dependent behaviour. It is therefore expected from the asymmetrical sourcedrain characteristics, that the $10 \mathrm{~s}, 20 \mathrm{~s}$ and $60 \mathrm{~s}$ plasma exposed F-CNTs devices will show field dependent behaviour when tested in backgated geometry. The 40 s plasma exposed F-CNTs device is not expected to show field dependence as there is little indication of semiconducting CNTs present on the sample. 


\subsubsection{Source-Drain Electronic Behaviour of N-CNTs}

The source-drain characteristics of the amine functionalised samples are shown in figure 6.10.

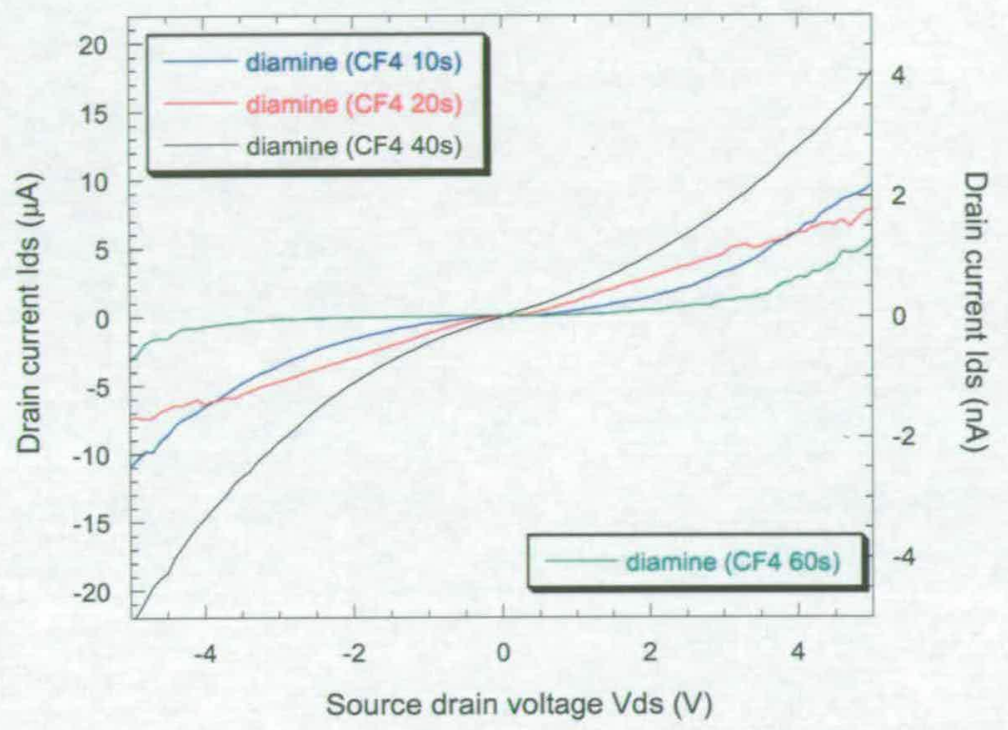

Figure 6.10: The source-drain characteristics for all $\mathrm{N}$-CNTs devices at $\mathrm{Vg}=O \mathrm{~V}$. The time indicates the exposure length of the CNTs to the initial $\mathrm{CF}_{4}$ plasma. The $60 \mathrm{~s}$ plasma exposed sample uses the right hand side $y$-axis, the $10 \mathrm{~s}, 20 \mathrm{~s}$ and 40 $s$ the left.

During the source drain sweep at $\mathrm{Vg}=0 \mathrm{~V}$, the $\mathrm{N}$-CNTs devices have again shown that the magnitude of the drain current varies between each device due to the statistical mix of CNTs on the devices. The 60 s plasma exposed N-CNTs device shows very low drain current of only a few $\mathrm{nA}$, whilst the other devices are all in the range of several $\mu \mathrm{A}$. All devices have shown a reduced magnitude of drain current in comparison to the F-CNTs at similar plasma exposure times.

The $10 \mathrm{~s}$ and $60 \mathrm{~s}$ plasma and diamine functionalised N-CNTs devices have both shown asymmetric source-drain characteristics indicating back to back Schottky diodes, similar to the F-CNTs devices. The $20 \mathrm{~s}$ plasma exposed N-CNTs device has shown a straight line through the origin implying a larger proportion of metallic CNTs, although the magnitude of the drain current and the gradient of the line implies a small bundle or a high resistance. The $40 \mathrm{~s}$ plasma exposed N-CNTs device again shows symmetrical resistor characteristics, although not as large a drain current as observed for the metallic $40 \mathrm{~s}$ F-CNTs device. 
It is predicted from the shape of the source-drain curves and the magnitude of the overall drain current, that the $10 \mathrm{~s}$ and $60 \mathrm{~s}$ N-CNTs devices are made from a mixture of metallic and semiconducting CNTs, whilst the $20 \mathrm{~s}$ and $40 \mathrm{~s}$ N-CNTs devices contain predominantly metallic CNTs. The N-CNTs devices were then tested in backgated geometry for field dependent behaviour and any indication of n-type doping of the CNT devices.

\subsubsection{Gate field dependent Electronic Behaviour of F-CNTs and N-CNTs}

Due to the problems in the positioning of the CNTs over the electrodes and the inconsistency between the bundles on each device, it is not possible to reliabley compare the backgated device characteristics for either the fluorine functionalised CNT devices nor the amine functionalised CNT devices. The quality of the devices produced after plasma functionalisation and diamine functionalisation are seen to vary across any given sample and the devices are not shown here. However as discussed previously this is believed to be due to the suspensions of the CNTs, used for drop coating, changing the average properties of each bundle. Although attempts were made in the drop coating process to prepare all samples by identical methods it is not possible to achieve $100 \%$ accuracy and identical samples, hence the varying magnitudes of drain current were observed.

A general trend was observed with p-type field dependent behaviour occurring after $\mathrm{CF}_{4}$ plasma exposure and ambipolar behaviour, post $\mathrm{CF}_{4}$ exposure and 1,2-diaminoethane functionalisation. The most apparent n-type behaviour is seen for the N-CNTs devices post $60 \mathrm{~s}$ plasma exposure, where there are fewer CNTs according to the magnitude of the drain current. However, the nature of the n-type effects cannot be fully correlated to the CNT stoichiometry in table 6.1, as the $\mathrm{N}$-CNTs device post $10 \mathrm{~s}$ plasma exposure shows mostly p-type conduction and no current increase in positive $\mathrm{Vg}$, even though the $\mathrm{N} / \mathrm{C}$ ratio is 0.065 . The $\mathrm{N}$-CNTs device post $20 \mathrm{~s}$ plasma exposure does have a limited increase at positive $\mathrm{Vg}$, but a high metallic leakage current. What can be said however, is that all the N-CNT field dependent devices have shown a reduced drain current in comparison to the similarly prepared F-CNTs devices, known to be the first stage of chemical doping $[85,86,130]$. The XPS data has confirmed that nitrogen has bonded to the F-CNTs in the form of a substitution reaction.

The decrease in the conduction with the diamine functionalisation is in agreement 
with the gate dependent characteristics shown by Valentini et al. [12]. However in the devices produced here the gate dependent effects are more pronounced and show a greater gate voltage dependence for the amine functionalised samples. In particular this can be attributed to the thickness of the gate oxides used. Here the oxide is $50 \mathrm{~nm}$, thick by comparison to state of the art devices, however the Valentini group have used a $250 \mathrm{~nm}$ thick thermal oxide and as such will only observed a small gate dependence over the ranges swept.

\subsubsection{N-type Doping of CNTs}

It is reasonable to attribute the observed n-type behaviour to the successful donation of electrons from the $\mathrm{NH}_{2}$ groups observed in the XPS spectra. The observation of n-type doping by nitrogen containing groups has been observed by other groups [15, $91,92,94,95]$ and is shown to be due to electron donation. In particular the success in the doping of CNTs by chemical methods has recently been realised by Klinke et al. [95] whereby exposing prefabricated CNT devices to hydrazine has doped the tubes from p-type to n-type. It was also shown that by chemically reacting dopants device characteristics such as the threshold voltage $\left(\mathrm{V}_{t h}\right)$ can be tailored. A similar technique by Auvray et al. [91] has pre-positioned CNTs by self-assembly onto an aminosilane monolayer and then exposed the devices to acidic or basic vapours. In turn this has removed or donated electrons making for $\mathrm{p}$ and $\mathrm{n}$-type devices to be fabricated.

As stated by Klinke [95] at moderate doping levels as is the case here, the barrier at the CNT electrode contacts dominate the conduction, whereas at high doping levels the carrier density dominates. Electrical modification has also been shown to take place by annealing the CNT devices in hydrogen [87] and in a vacuum $[85,86]$. The vacuum annealing study [85] produced ambipolar tubes by selectively annealing specific stages along a single tube using a PMMA mask.

Previous CNT device annealing studies also confirmed the difference between fieldeffect behaviour due to the contact between the CNT and the electrode and due to sidewall doping contributions $[85-87,95]$. In particular the conventional doping will cause a shift in the threshold voltage of the device, whereas contact annealing (which acts to shift the Fermi level) shows no threshold voltage shift. This results in the fact that by annealing to remove the excess oxygen, ambipolar devices can be made. 
For the devices produced here the CNT/electrode contacts are likely to dominate the conduction. It is proposed that due to measurements taking place in air with no annealing stage, the charge carriers on the CNTs can become unsettled and may in fact drift over time. Importantly, the influence of atmospheric oxygen altering the electronic response should not be ruled out. The noise observed on the drain current may be indicative of defect sites having been created on the CNTs. Also it is reasonable to presume that when conduction takes place through a network of CNTs that electrons and holes may have to overcome potential barriers from other tubes and the functional attachment groups on the CNT sidewalls.

However, in the case of both the F-CNTs characteristics and the N-CNTs, there appeared to be a shift in the position of $\mathrm{V}_{t h}$ for both types of devices. The shift is more pronounced after diamine functionalisation at $20 \mathrm{~s}$ plasma exposure and $60 \mathrm{~s}$ plasma exposure. The diamine functionalisation has also been shown to reduce the ability of hole carriers as is known to be the first stage of n-type doping $[12,15,92]$.

\subsubsection{Device Characterisation}

As discussed in Chapter 3 the field dependent devices can be characterised by studying the Schottky barriers, the subthreshold behaviour and the carrier mobilities. However, the devices made here have several problems when such analysis is considered as discussed in Sections 6.5.2 and 6.5.3. Shown in figure 6.11 is a logarithmic plot of the drain current vs the source-drain voltage, from Sections 6.5 .2 and 6.5 .3 for both the F-CNTs devices and the N-CNTs devices respectively, showing the devices are back to back Schottky diodes as discussed.

In particular it can be seen for the fluorine devices, figure 6.11(a), that the magnitude of the current varies by several orders of magnitude between the $10 \mathrm{~s}$ and $20 \mathrm{~s}$ plasma exposure times and the $40 \mathrm{~s}$ and $60 \mathrm{~s}$. All measurements were taken at zero gate voltage. The devices are not directly comparable as it is not possible to say how many CNTs are between the electrodes for each device. Similarly, for the diamine functionalised devices, figure 6.11(b) the same problems occur. The magnitude of the drain current is similar between each devices, making a more useful comparison with the exception of the devices aminated after $60 \mathrm{~s}$ plasma exposure. 


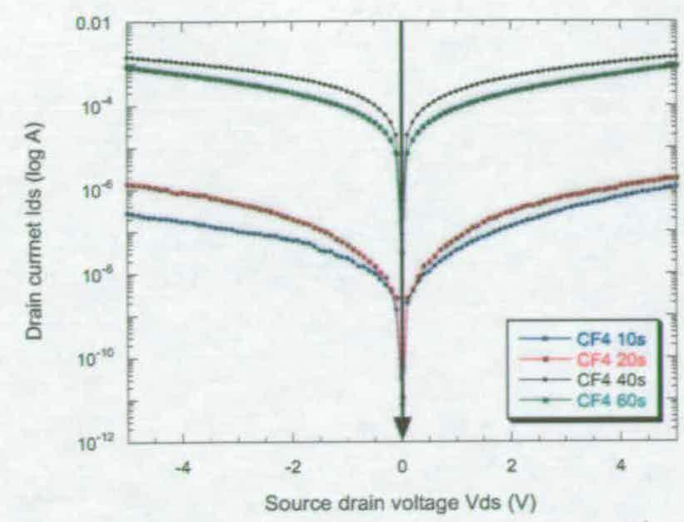

(a) The logarithmic output of the F-CNTs sourcedrain characteristics

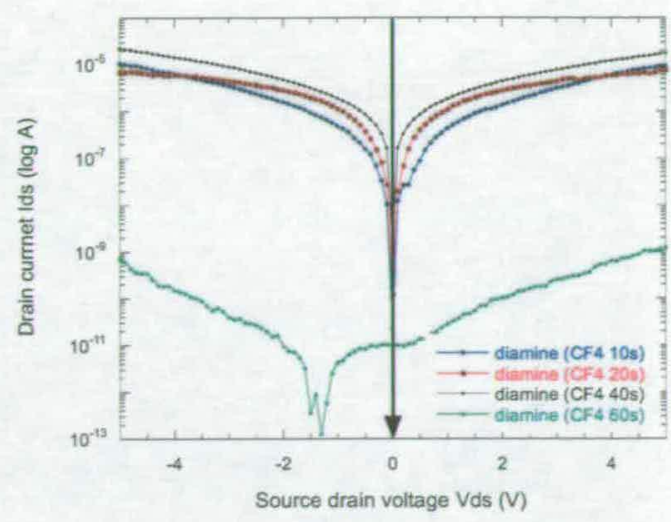

(b) The logarithmic output of the N-CNTs sourcedrain characteristics

Figure 6.11: A logarithmic plot of the drain current vs source drain voltage for the F-CNTs and $\mathrm{N}$-CNTs devices. $\mathrm{Vg}=\mathrm{OV}$ for all device shown. The time indicates the length of the original plasma exposures.

As field dependent devices, we can state that p-type or n-type behaviour has been observed, but determining the ideality factor, $\eta$ and the Schottky barrier height $\phi_{B}$ is not possible, as the area of the contact between the CNT and electrode can not be defined, nor can the saturation current be used in a truly comparative method. It is highly likely that some level of metallic tubes remains in the device, thus also rendering the extraction of transistor characteristics an unreliable means of characterisation.

\subsubsection{Summary of the I-V characteristics}

The devices produced by the functionalisation of $\mathrm{CNTs}$ in a $\mathrm{CF}_{4}$ plasma followed by further amine-functionalisation have shown altered gate dependence between purely p-type and towards n-type respectively. The amine donation of electrons to the CNT conduction has decreased the hole carrier contribution and allowed for electrons to flow under the application of a positive gate bias. As the plasma exposure time was increased it became more likely for the CNT devices to breakdown when a higher Vds was applied as opposed to burn off to a semiconducting device. However, the statistical differences in the CNT mixtures between the electrodes has made a direct comparison of device behaviour difficult.

Nevertheless, it can be stated that the plasma fluorination is a successful means as a first step to create n-type CNT devices, with the degree of diamine functionalisation 
being directly dependent on the fluorination beforehand.

\subsection{Conclusion}

In summary it has been shown that substitution reactions of 1,2-diaminoethane onto plasma fluorinated CNTs has been successful. The reaction is limited by the initial fluorination of the CNTs themselves and it is shown that the ratio of nitrogen observed on the CNTs increases following the trend for the initial fluorination of each sample.

The ratio of the primary amines to secondary amines has been shown to increase with initial fluorine content implying that bi-functionalisation has taken place. The overall nitrogen content as a function of initial fluorine content was seen to decrease confirming that the replacement of fluorine with 1,2-diaminoethane molecules took place at a ratio of less than 1:1.

The devices produced by the functionalisation of CNTs in a $\mathrm{CF}_{4}$ plasma followed by further diamine-functionalisation have shown altered gate dependence between purely p-type and towards n-type respectively. The amine donation of electrons to the CNT conduction has decreased the hole carrier contribution and allowed for electrons to flow under the application of a positive gate bias. As the plasma exposure time was increased it became more likely for the CNT devices to breakdown as opposed to burning off to a semiconducting device. However, the magnitude of the drain current was largely affected by the mixing of CNTs between the electrodes and was prone to variations between devices.

The plasma fluorination is a successful means as a first step to create n-type CNT devices, with the degree of diamine functionalisation being directly dependent on the fluorination beforehand. The devices made here have shown improvements from similar plasma fluorinated and further amine functionalised CNT devices made by Valentini et al. [11]. However, the devices made were not as refined as those made by the IBM group with direct sidewall functionalisation processes $[85-87,95]$.

By refining the functionalisation procedures, both during the fluorination and the diamine functionalisation, the doping effects may be controlled. In particular, by achieving a more covalent $\mathrm{C}-\mathrm{F}$ bond it is predicted that the $\mathrm{N} / \mathrm{C}$ ratio after the diamine func- 
tionalisation can be increased. All further functionalisation procedures to be considered must maintain the structural integrity of the CNTs to exploit the electronic behaviour. $\mathrm{SF}_{6}$ plasma exposure has indeed shown the ability to create more covalent $\mathrm{C}-\mathrm{F}$ bonds without loss of the CNT structure and is therefore predicted to be a useful first step for future functionalisation experiments.

It is therefore envisaged that controllable CNT devices can be produced by using the plasma fluorination and controlled functionalisation procedures. Altered fabrication procedures such as electrode annealing should also improve the electrical behaviour of the doped CNT devices. 


\section{Chapter 7 \\ Plasma Thiolation of Carbon Nanotubes for Self Assembly}

It is widely accepted that in order to successfully use carbon nanotubes (CNTs) for electronic devices a room temperature method to enable the selective positioning of already grown CNTs is needed. A positioning method could be implemented either by selectively making a substrate reactive, as will be discussed in Chapter 8 or by functionalising the normally unreactive CNTs themselves.

The functionalisation of CNTs has been an intense topic of research in the current scientific literature, where in particular CNTs have been treated to long acid exposures to produce carboxylic acid end groups [34,39], and as presented in the previous chapter, fluorinated via gas fluorination steps. Many of these functionalisation processes show exceptional promise for uses in material science, as the CNTs can be more easily dispersed and introduced into nanocomposite materials, but are not so promising for selectively positioning CNTs. For selective positioning, thiol bonding shows the most promise.

The plasma thiolation process developed here will be discussed in the following chapter, including experimental evidence of CNT self-assembly and electronic characterisation of the functionalised CNTs.

\subsection{Thiolation of Carbon Nanotubes, a Brief Review}

The Au-thiol bond is known to be selective and occur spontaneously in solution, provided that the gold surface is clean and of high quality. Reports have demonstrated thiol functionalisation techniques using long and short chain alkanethiols respectively [42, $122,125]$.

To take advantage of the thiol gold self-assembly, CNTs had been previously thiolated by 
substitution reactions. In all cases CNTs were firstly functionalised by long acid purification and cutting steps to obtain carboxylic acids [33-35, 39]. Smalley et al. have functionalised the CNTs with a long chain carbon group to produce CNT- $\left(\mathrm{CH}_{2}\right)_{11}-\mathrm{SH}$ [33], whilst Liu et al. produced CNT-CONH- $\left(\mathrm{CH}_{2}\right)_{2}-\mathrm{SH}$ [34]. A shorter chain reaction was then developed by Lim et al. [35] where via several acid reflux steps, conversion of carboxyl groups to hydroxyl groups, further conversion of hydroxyl groups to chloride groups, and finally thiolation, $\mathrm{CNT}-\mathrm{CH}_{2}-\mathrm{SH}$ tubes were produced.

Here, the direct thiolation of single-walled CNTs, without any long chain carbon groups, e.g. CNT-SH was achieved, by physically mixing elemental sulphur with CNTs and then exposing the sulphur/CNT mixture to an $\mathrm{Ar} / \mathrm{H}_{2}$ plasma.

The advantage of this method has been to allow CNTs to attach selectively to gold electrodes with a short functionalisation time. Due to the non-destructive nature of plasma functionalisation processes $[11,13,17,18,147,148]$, and Chapters 5 and 6 , it was envisioned that a thiol rich plasma environment could be created allowing for direct thiolation of CNTs. The sulphur based plasma has demonstrated the creation of thiolated CNTs and the subsequent self-assembly across patterned gold electrodes.

\subsection{Sulphur Plasma Exposure of CNTs}

\subsubsection{Experimental procedure}

Single-wall carbon nanotubes (CNTs) from Carbon Nanotechnologies Inc were used in these experiments. The sulphur/CNTs samples were prepared by physical mixing with elemental sulphur from Aldrich. The non-treated sample, sample 1, is referred to as the control sample throughout the text.

Other samples were then exposed to an argon and hydrogen based plasma mixture within the Plasma Etch reactor with the electrodes heated to $120^{\circ} \mathrm{C}$ to allow the sulphur to melt, the plasma feed stock gas flows and sulphur/CNTs mixing ratios are outlined in table 7.1. All exposures were carried out with an R.F. power of $250 \mathrm{~W}$. After stabilisation of the gas flows the sulphur/CNTs mixtures were exposed to the plasma for 1 minute. A schematic of the plasma setup is shown in figure 7.1.

The localised blue region of the plasma was clearly visible through a quartz window 


\begin{tabular}{cccc}
\hline \hline Sample & Weight of sulphur/CNT $(\mathrm{g})$ & Ar flow $(\mathrm{sccm})$ & $\mathrm{H}_{2}$ flow $(\mathrm{sccm})$ \\
\hline Control & $0 / 0.002$ & No plasma & No plasma \\
1 & $0.02 / 0.002$ & No plasma & No plasma \\
2 & $0.02 / 0.002$ & 75 & 25 \\
3 & $0.02 / 0.002$ & 50 & 50 \\
4 & $0.02 / 0.002$ & 25 & 75 \\
\hline
\end{tabular}

Table 7.1: The reactor conditions. The reactor was heated to $120^{\circ} \mathrm{C}$, with R.F. power 250 $W$ and plasma exposure time of 1 minute

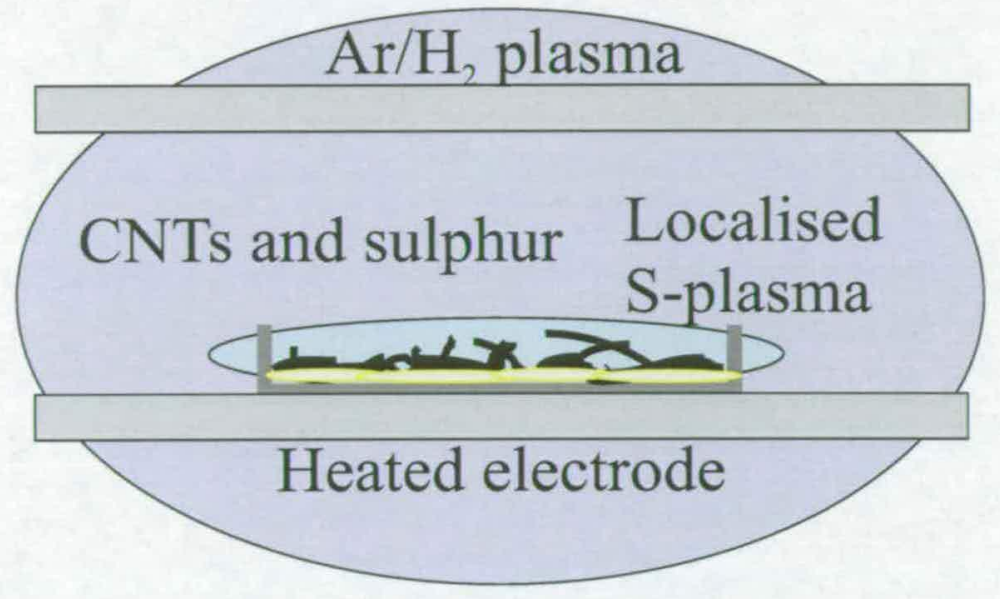

Figure 7.1: A schematic representation of the plasma thiolation set-up

around the region where the sulphur/CNTs samples had been placed. The blue colour is indicative of a sulphur based plasma, whilst the shown purple colour is representative of the $\mathrm{Ar} / \mathrm{H}_{2}$ mixture.

Immediately after plasma exposure (where exposure had taken place) the samples were placed in $2 \mathrm{ml}$ of toluene and rinsed through with $5 \mathrm{ml}$ of toluene, via a $2 \mu \mathrm{m}$ pore sized PTFE filter from Millipore, to rid of any excess sulphur. The CNTs were then drop-coated onto a silicon wafer for characterisation.

To characterise the extent of sulphur attachment to the nanotubes before self-assembly, X-ray photoelectron spectroscopy (XPS) and Raman spectroscopy have been used. 


\subsubsection{The XPS Spectra of Thiolated CNTs}

It has been discussed previously, Chapter 5, that CNTs exposed to fluorinated plasmas for time periods of several minutes do not suffer significant damage to the structure, as shown by Raman spectroscopy. It was verified by XPS that several minutes is sufficient exposure time in such a reactive atmosphere for functional groups to attach to the sidewalls of CNTs.

XPS was performed on the thiolated CNTs with emphasis on the S2p peaks for the control CNTs, CNTs that have been mixed with sulphur (sulphur/CNTs) and sulphur/CNTs mixture exposed to $\mathrm{Ar} / \mathrm{H}_{2}$, figure 7.2. The peaks have been fitted using XPS peak software, with $\Delta \mathrm{E}=1.18 \mathrm{eV}$ between the $\mathrm{S}_{\mathrm{P}_{3 / 2}}$ and $\mathrm{S}_{\mathrm{p}_{1 / 2}}$ and fixed intensity ratio of $2: 1$ respectively. A mixture of Lorentz:Gaussian peaks were required at 70:30, with FWHM of 1 as in the literature $[122,125]$. It has been observed in these experiments, that for all CNT samples exposed to sulphur treatment, a binding energy of 163.7-163.8 eV was found.

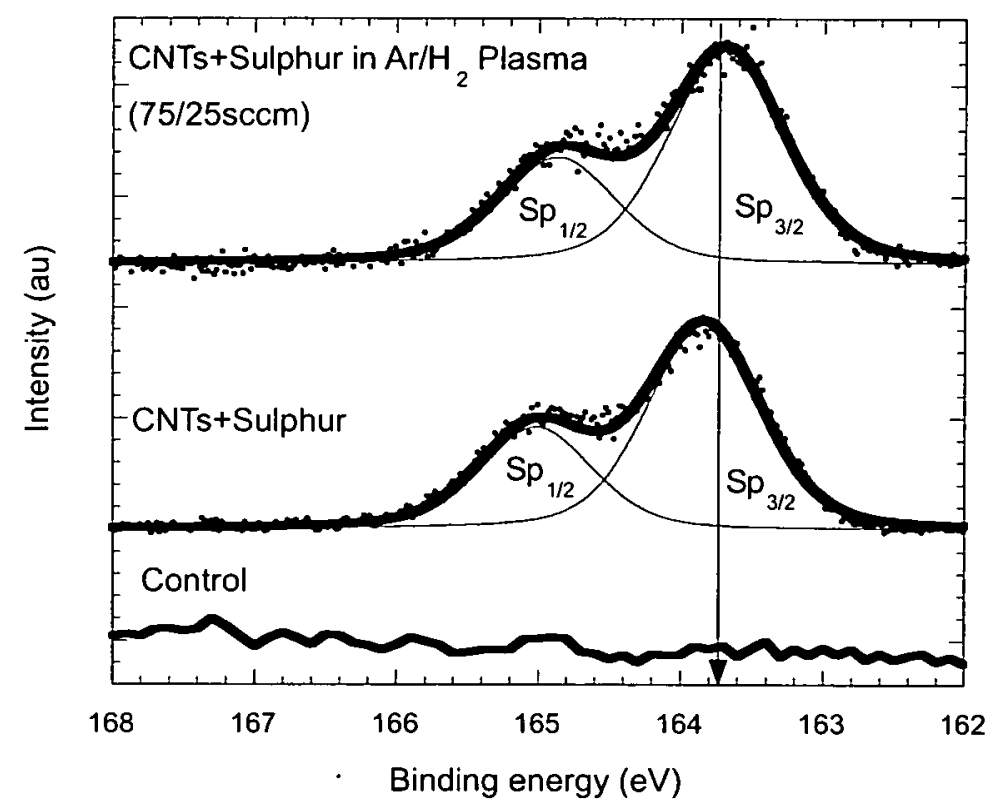

Figure 7.2: The S2p peaks of the control CNTs, sulphur/CNTs and sulphur/CNTs exposed to $\mathrm{Ar} / \mathrm{H}_{2}$ plasma

According to the literature, $\mathrm{S}-\mathrm{H}$ is present at $163.7 \mathrm{eV}$ while S-S occurs at $163.8 \mathrm{eV}$ and S-Au bond is known to occur at $162 \mathrm{eV}[122,124,125]$. Multilayer thiols have also been observed at $164.4 \mathrm{eV}$ [162] and $163.6 \mathrm{eV}$ [124]. Due to the closeness of 
the energy peaks for sulphur containing compounds, we conclude that some form of sulphur is bonded to the CNTs after sulphur treatment within the thiol region. There is also a general trend for the position of the $S 2 p$ thiol peak to shift to higher binding energies with an increase in the length of the carbon chain attached to the thiol [42]. As the CNTs are exceptionally long carbon groups, a slightly higher S2p peak position is to be expected.

Figure 7.3 shows the influence of the flow rate of $\mathrm{H}_{2}$ in the $\mathrm{Ar} / \mathrm{H}_{2}$ gas mixture during plasma exposure on (a) the $\mathrm{S} / \mathrm{C}$ ratio and (b) the position of the S2p energy peak. The amount of S/C (around 5\%) does not appear to be affected significantly by the presence of the plasma nor by the rate of $\mathrm{H}_{2}$ flow, yet the presence of the plasma does shift the S2p peaks to slightly lower binding energies. Therefore, we see that the exposure to the $\mathrm{Ar} / \mathrm{H}_{2}$ plasma is not essential for sulphur to attach to the CNTs but does help to provide an excess $\mathrm{H}_{2}$ environment for thiolation to take place.

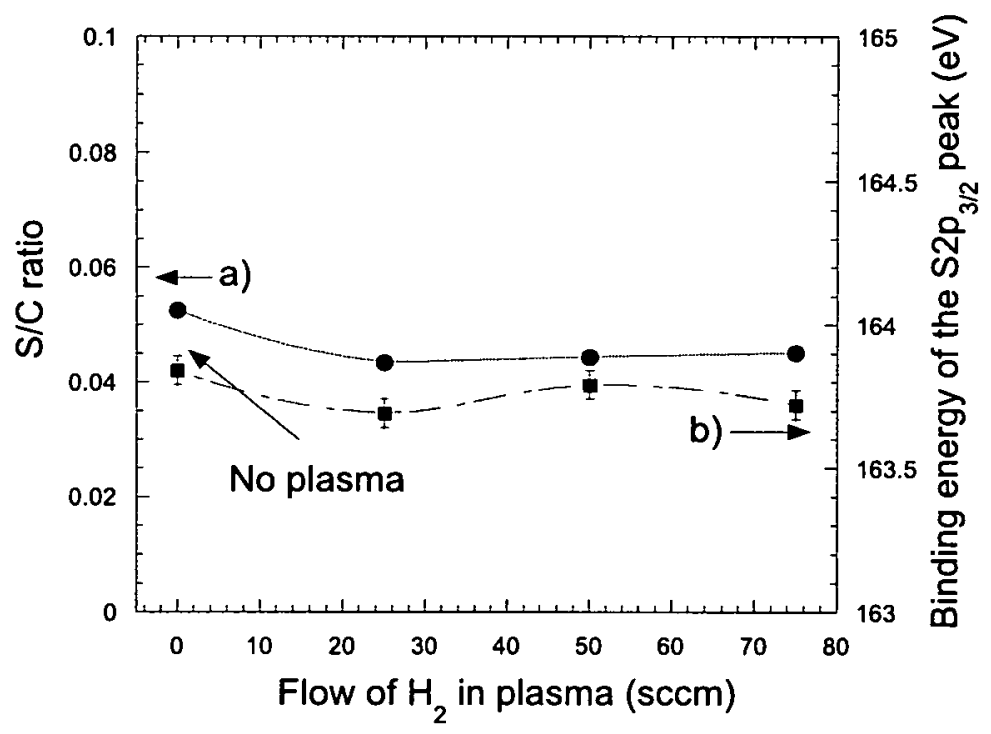

Figure 7.3: The influence of the $\mathrm{H}_{2}$ flow rate in the $\mathrm{Ar} / \mathrm{H}_{2}$ plasma exposure (total flow = $100 \mathrm{sccm}$ ) on (a) the $S / C$ ratio and (b) the position of the $S 2 p$ energy peak of the sulphur/CNTs. When at $\mathrm{H}_{2}=0 \mathrm{sccm}$, no plasma has been struck.

A level of confidence can be given to the assignation of the S2p peaks for the plasma exposed CNTs and that complete thiolation was achieved due to the observed spin orbit coupling. During the peak fit, the $\mathrm{S} 2 \mathrm{p}$ regions were fitted with $\mathrm{Sp}_{3 / 2}: \mathrm{Sp}_{1 / 2}$ of $2: 1$ without the addition of further peaks. If indeed an overlap between CNT-S-S-CNT and CNT-SH has occurred, deconvolution by a single peak would not be possible [124]. 
Plasma functionalisation of graphite and glassy carbon using $\mathrm{SO}_{2}$ plasma have shown similar sulphur atomic percentages and position of the S2p peaks, at D.C. bias voltages and exposure times close to those used in these experiments [163]. Moreover, the adhesion of sulphur in the thiol region without the presence of additional S-O peaks is demonstrated in our thiolation method, even after 4 weeks exposure to atmosphere. The $\mathrm{SO}_{2}$ bonding, often found in different methods of treating the CNTs $[34,35]$ and other forms of carbon $[42,126,164]$, is observed by others usually at $167 \mathrm{eV}$. In addition, the oxygen peak component has not been seen to increase in our thiolated CNTs after 4 weeks exposure to atmosphere, as has been the case in our previous studies on the fluorine functionalisation process $[17,18]$.

Experimental evidence suggests that the lack of oxygen incorporation into the CNTs is inherent to our thiolation process. Unfortunately no plasma diagnostic tools were available for these experiments, but a presumption can be made that by the lack of $\mathrm{SO}_{2}$ peak especially after the time interval of 4 weeks has elapsed, that the plasma created defect sites were directly functionalised by the SH plasma in the reactor.

\subsubsection{The Raman Spectra of Thiolated CNTs}

The Raman spectra for the control CNTs, sulphur/CNTs and sulphur/CNTs exposed to $\mathrm{Ar} / \mathrm{H}_{2}$ plasma are shown in figure 7.4. The presence of $\mathrm{D}$ and $\mathrm{G}$ bands and the RBM modes indicate that the tubular structure of the nanotubes have not been destroyed by these plasma treatments, figure 7.4(a) and that the average tube diameter distribution ranges from $0.8-1.3 \mathrm{~nm}$ by the simple calculation [32]

$$
d_{t}=\frac{248}{\omega_{R B M}}
$$

discussed in Chapter 3.1 .

A zoomed in view of the Raman spectra between 500 and $1200 \mathrm{~cm}^{-1}$ show low intensity peaks inherent to the Raman spectra of carbon nanotubes $[110,152]$ are evident in all three samples, figure $7.4(\mathrm{~b})$. Neither the $S=C$ peak at $1095 \mathrm{~cm}^{-1}$ nor $S-S$ peak at $585 \mathrm{~cm}^{-1}$ have been observed after the plasma thiolation treatment, in contrast to results reported by Lim et al. [35]. The lack of $S$ as indicated by Raman in our experiments could be due to the small sulphur atomic concentration measured, around $5 \%$ 


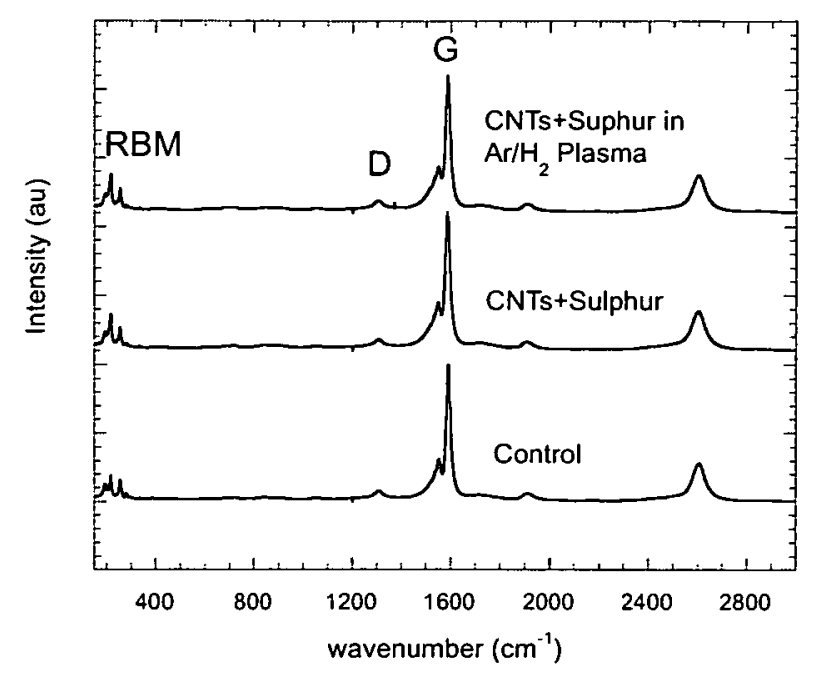

(a) The whole spectra

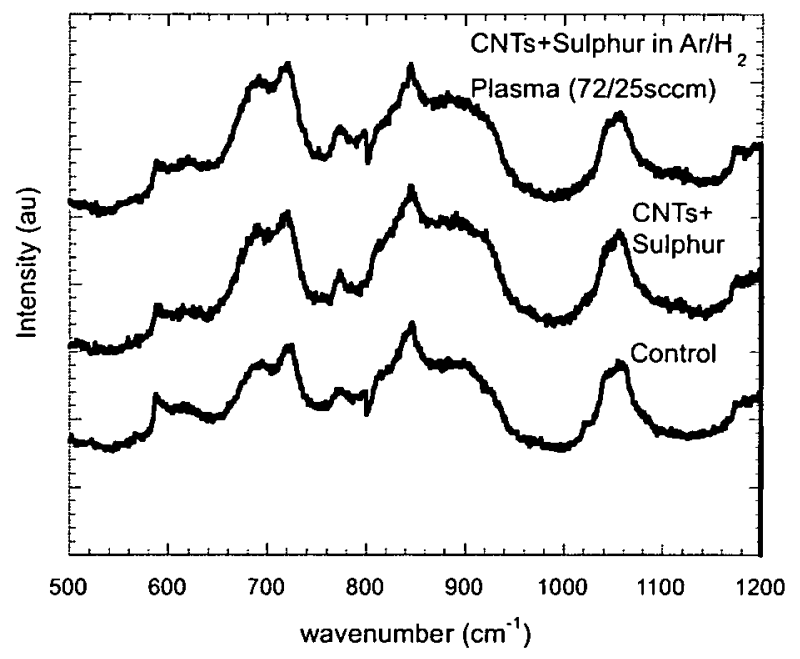

(b) A zoomed in view over $500-1200 \mathrm{~cm}^{-1}$

Figure 7.4: The Raman spectra of control CNTs, sulphur/CNTs and sulphur/CNTs exposed to $\mathrm{Ar} / \mathrm{H}_{2}$ plasma

by XPS experiments, whereas in the method used by Lim et al., the sulphur content is expected to be higher [35].

Other work where carbon films and carbon rods have been doped with sulphur resulting in atomic percentages similar to those observed in our experiments also show no sulphur related peaks in the Raman spectra [123, 165-168]. 


\subsubsection{Direct Thiolation Summary}

The plasma thiolation process has been successful in the production of an environment capable of attaching thiols to CNT sidewalls. The heating of the sulphur and the addition of a $\mathrm{H}_{2}$ feed stock gas were both essential in creating such an environment as without either CNTs were not thiolated. The plasma chemistry has also been advantageous for the thiolation without the addition of excess of oxygen contaminants which are known to alter the electrical behaviour of the CNTs.

\subsection{Self-assembly of Thiolated Carbon Nanotubes}

The directly thiolated CNTs have been self-assembled onto predefined Au electrodes. As the XPS has indicated the presence of sulphur in the thiol region, the self-assembly is presumed to be due to the spontaneous reaction between the gold and thiol bond. Such that

$$
S H+A u \longrightarrow S A u+H
$$

\subsubsection{Self-Assembly Experimental Procedure}

For the self-assembly of thiolated CNTs, a drop of functionalised CNT solution was placed in $2 \mathrm{ml}$ of 1,2-dichloroethane and sonicated for 10 minutes. The 1,2-dichloro ethane was used as the suspension solvent for the CNTs as the $\pi$ bond in the ethane molecule is known to attract the $\pi$ bonds of the CNT surface allowing for better suspensions compared to other common solvents such as IPA.

Electrode structures which had been fabricated by e-beam lithography and e-beam evaporated $\mathrm{Ti} / \mathrm{Au}(5 / 25 \mathrm{~nm})$ were cleaned by sonicating in ethanol then removing the samples and heating in an oven at $120^{\circ} \mathrm{C}$ for 20 minutes. The cleaned electrode samples were then left in the functionalised CNTs solution and covered for 12 hours. The samples were then removed, cleaned by ethanol rinsing and viewed in the Hitachi S4500 scanning electron microscope (SEM).

The thiolated CNTs described as sample 2 in table 7.1, whereby plasma exposure for 
1 minute was performed at 250 W R.F. power, and feed stock gas of $\mathrm{Ar} / \mathrm{H}_{2} \quad 75 / 25$ sccm respectively, were used for the self-assembly process. Both HiPCO CNTs from CNI and laser ablation CNTs made by Zhang [169] at NEC were subjected to identical plasma treatment and self-assembly tests. Spectroscopic data ${ }^{1}$ was in agreement with the earlier discussion showing that the growth method of the CNTs did not alter the plasma thiolation properties.

\subsubsection{Scanning Electron Microscopy}

Figures 7.5 and 7.6 both show the self-assembly of thiolated CNTs across Au electrode structures, by SEM characterisation. Several authors have indicated the end groups of CNTs to be the most reactive part of the CNTs $[2,31,34,35]$.

The low stoichiometries of the sulphur attachment shown in figure 7.3 support the hypothesis that the sulphur will not have bonded along the entire length of the CNTs. The self-assembly is therefore thought to be due to the $\mathrm{SH}$ bond being at the more reactive ends of the CNTs. The observed nature of the self-assembly is for the CNTs to bridge the defined electrode structure as clumps of CNTs, see figures 7.5 and 7.6. The HiPCO CNTs show larger clumps on average than the laser ablation CNTs.

The reaction dynamics of the self-assembly system is thought to depend on the energetic balance between the S-Au bond and the mechanical properties of the CNT bundles. Due to the length of the CNTs the reaction dynamics will have shifted towards the intermolecular forces between the CNTs rather than the thiol gold interaction.

CNTs were also seen to have self-assembled onto the larger gold pads. There was little example of adhesion of CNTs to the $\mathrm{SiO}_{2}$ surface although the presence was not $100 \%$ exclusive. In comparison, the sulphur/CNT non-plasma exposed sample has shown no sign of self-assembly to the gold electrodes nor any surface physisorption effects after the self-assembly stage.

\footnotetext{
${ }^{1}$ Not shown as is only a repeat of the earlier discussion.
} 


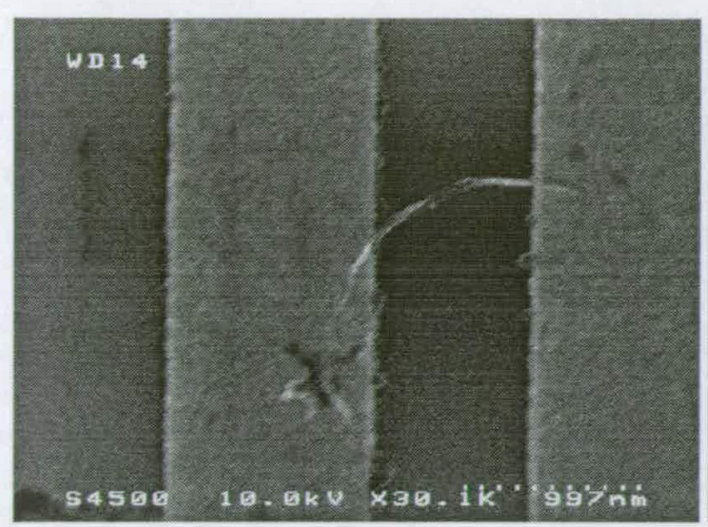

(a)

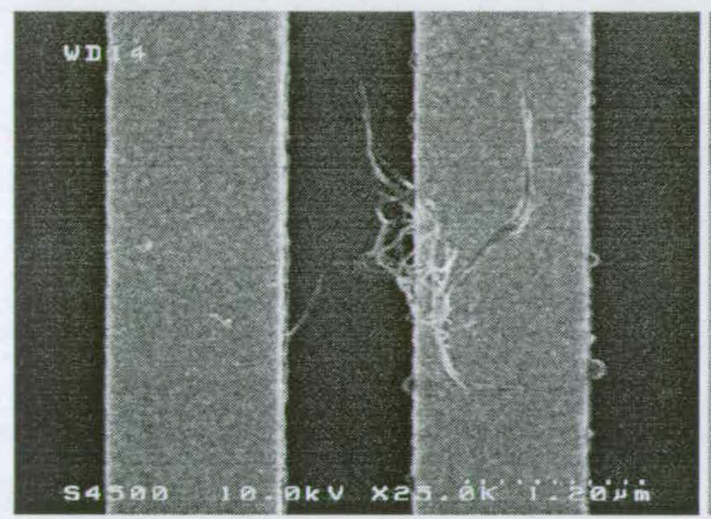

(c)

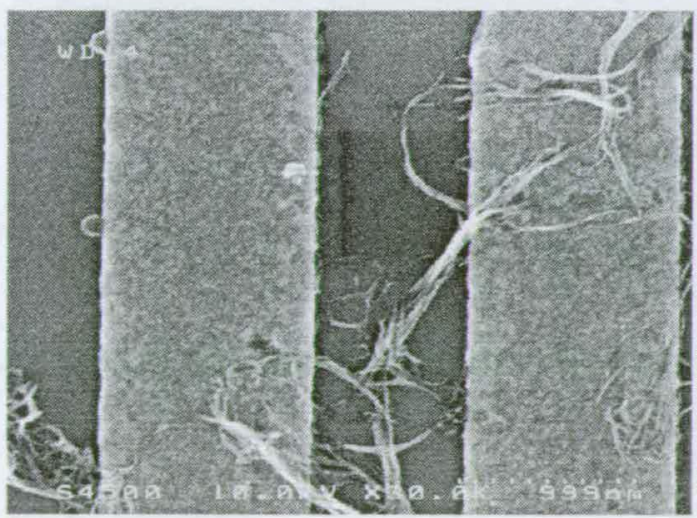

(e)

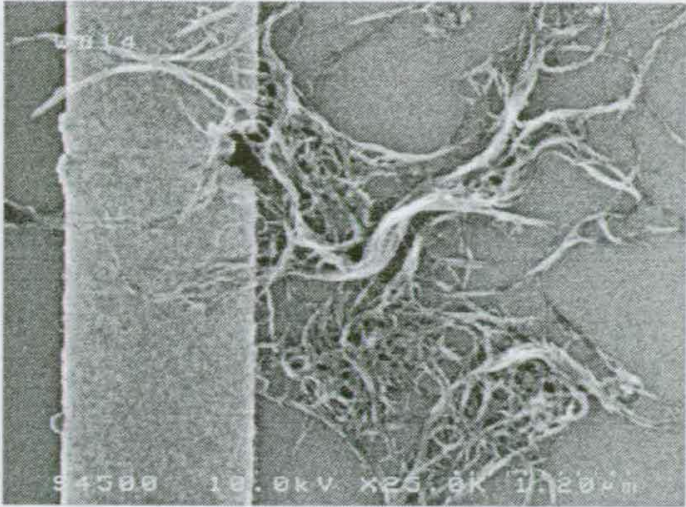

(b)

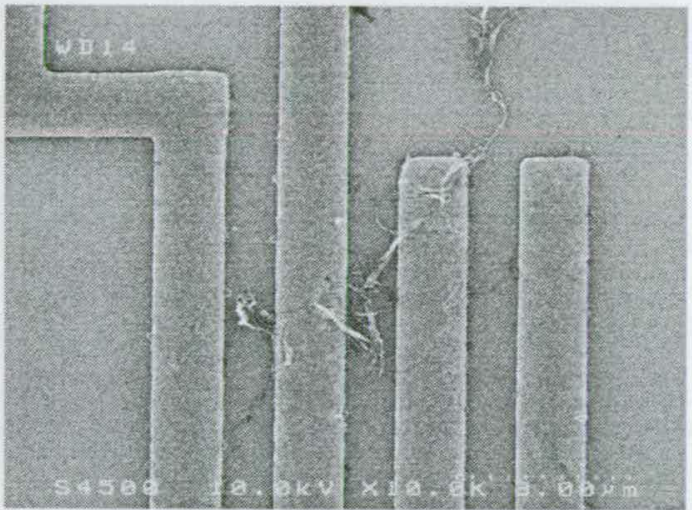

(d)

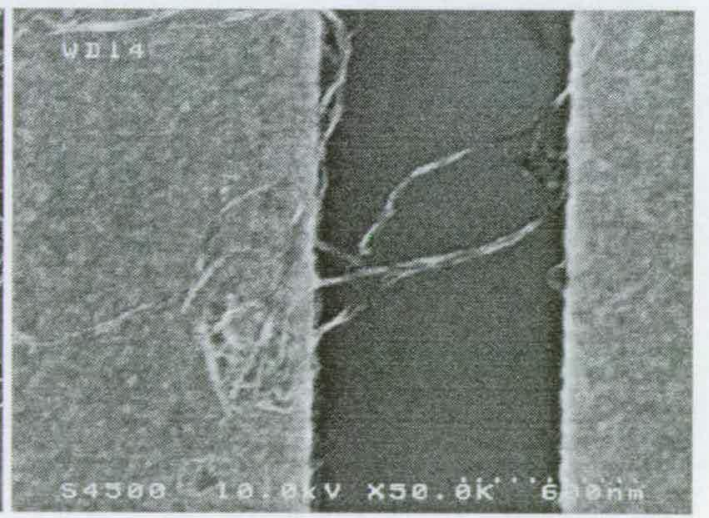

(f)

Figure 7.5: Examples of HiPCO plasma thiolated carbon nanotubes bridging Au electrodes. Six examples of the CNTs bridging the electrodes are shown in figures (a)-(f). The CNTs are visibly bundled together. 


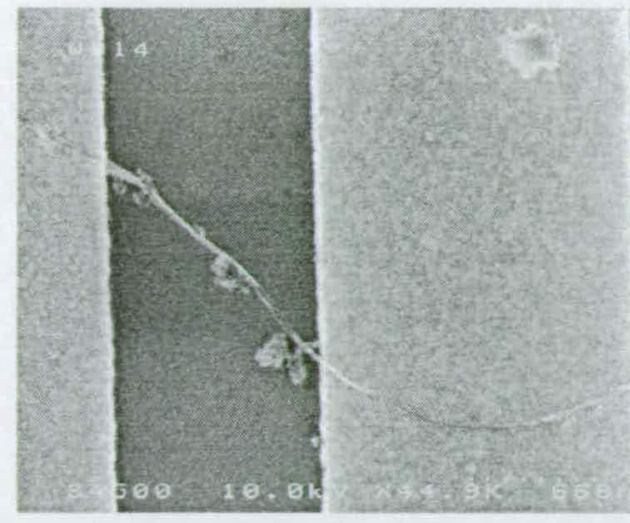

(a)

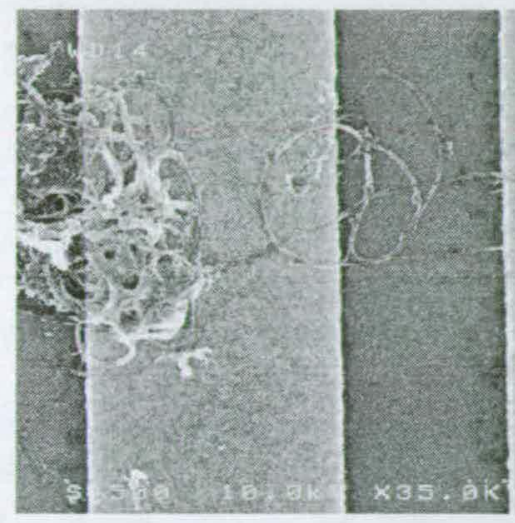

(c)
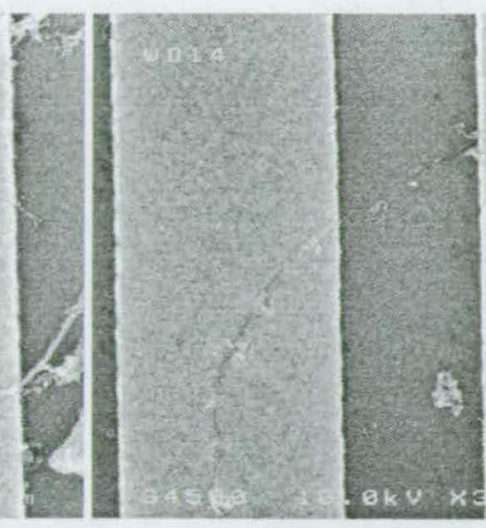

(b)

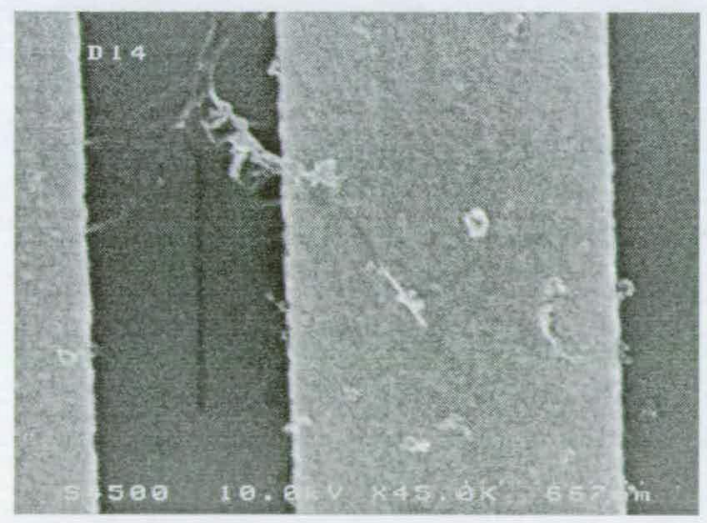

(e)

Figure 7.6: Examples of laser ablation plasma thiolated carbon nanotubes bridging $A u$ electrodes. Five examples of the CNTs bridging the electrodes are shown in figures (a)-(e). The CNTs are visibly bundled together: 
As an initial test of the CNT self-assembly, a $1 \mathrm{~cm}^{2} \mathrm{SiO}_{2}$ chip was lithographically patterned with $\mathrm{Au}$ trenches and the self-assembly process tested. However, there was no possibility of backgating these device for further electronic characterisation. The CNT dispersion solvent in this case was ethanol. Figure 7.7 shows both low and high resolution scanning electron micrographs of the functionalised CNTs lying across the gold trenches after self-assembly.

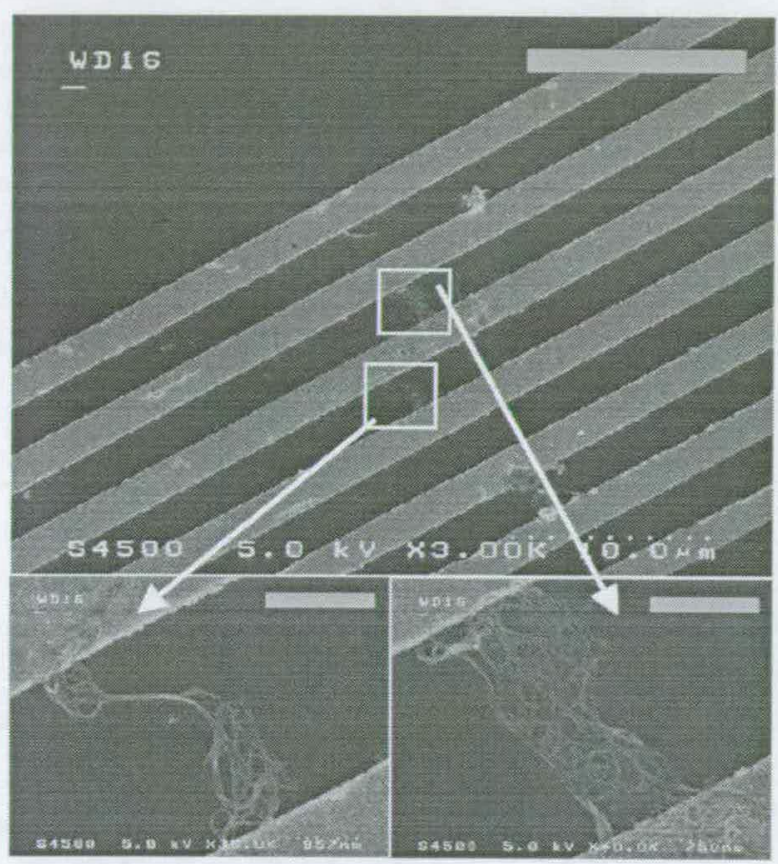

Figure 7.7: An SEM image of the sulphur/CNTs exposed to $\mathrm{Ar} / \mathrm{H}_{2}$ self assembled across gold trenches

The CNT bundles are seen to align across the trenches as opposed to along the trenches. The bundles appear on average to be larger than those observed when CNTs are suspended in 1,2-dichloroethane. As discussed above the thiolation is more likely to have occurred at the end of the CNTs. The reaction dynamics indeed shows a definite favouring of CNTs bridging electrodes. The previously thiolated CNTs made by Liu et al. [34] has shown the CNTs to self-assemble vertically, e.g. perpendicular to the Au surface. There the CNTs had been shortened during the aggressive acid functionalisation stages required for the thiolation process. As such, the Liu process would not be as suitable for the CNT devices produced here.

The reaction dynamics of the direct thiolation system was also seen to be dependent upon the initial suspension of the CNTs in the solvent. There was a trade off between 
the self-assembly of the CNTs and the bundling together within the solution after sonication. If the CNTs were not well suspended no self-assembly took place and the sample surface showed only large bundles of CNTs which were merely physically absorbed to the surface.

\subsubsection{Self-Assembly Summary}

The SEM images have shown that both HiPCO CNTs and laser ablation CNTs can be self assembled across gold electrodes after direct plasma thiolation. The reaction dynamics of the self-assembly process in relation to the chain length of the carbon nanotubes appear to have been advantageous in these experiments, allowing the CNTs to bridge $\mathrm{Au}$ electrodes over an oxide region.

\subsection{Electrical Characterisation of Plasma Thiolated Carbon Nanotubes}

Thiolation of single-walled CNTs has been demonstrated whereby the ends of the nanotube bundles have been selectively functionalised making it favourable for the nanotubes to self-assemble across a substrate to bridge gold electrodes.

\subsubsection{Electronic Testing: Experimental Procedure}

To characterise the electronic behaviour of the thiolated CNTs, the HP4156B probe analyser was used. The CNTs had been self-assembled following the procedures outlined previously in Section 7.3.

When only two contacts were available the CNT films were probed between a source and drain voltage. Where backgated devices were fabricated, the CNTs were tested between a source, drain and gate probe as described in Chapter 3.3.

\subsubsection{Thiolated CNT Bulk Film Behaviour}

Films of directly thiolated CNTs have been assembled over gold electrodes on $\mathrm{SiO}_{2}$ in a similar method to the self-assembly process. The electrical behaviour of the bulk 
film has shown an average resistance of $15454 \mathrm{Ohms}$ at $10 \mathrm{~V}$, figure 7.8 and is shown in comparison to a randomly dispersed bundle of pristine CNTs. Such a current is higher than that observed for single CNTs bridging electrodes by electric field alignment methods $[81,82]$ as there are many more CNTs present.

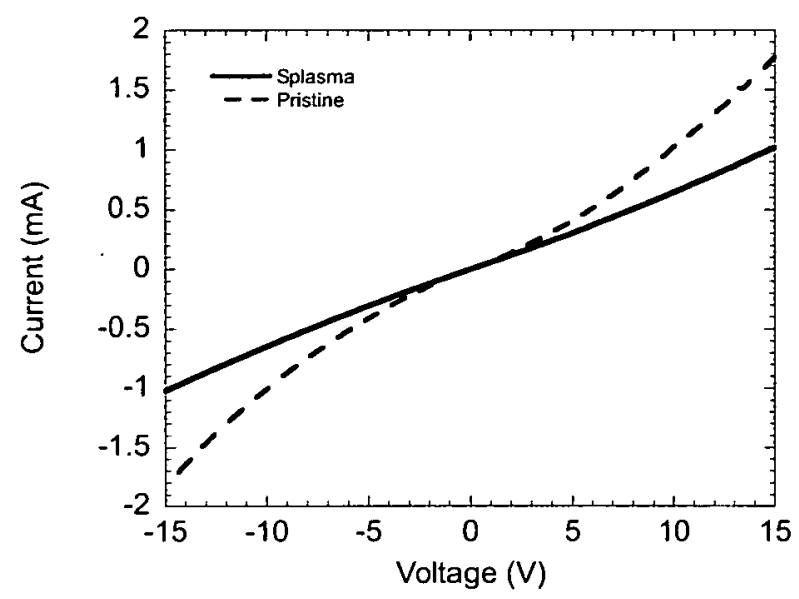

Figure 7.8: The source-drain characteristics of a self-assembled S-CNTS bundle produced at room temperature, and a randomly dispersed bundle of pristine CNTs.

However, it is not possible to say how many CNTs are present, nor directly relate these results to bulk behaviour of CNT films due to the statistical differences in any CNT suspension, a recurring problem throughout this study. As discussed in Section 7.3, the samples ought to be backgated for electronic characterisation, which has been done with the 1,2-dichloroethane suspended CNT samples.

\subsubsection{Back-gated Thiolated CNT Device Behaviour}

As a comparison between the different CNTs, devices have been made with both HiPCO carbon nanotubes from CNI which were purchased with a presumed acid purification steps and also laser ablation carbon nanotubes made within the NEC labs [169]. Where dense CNT solutions were produced and large bundles of CNTs observed metallic conduction dominated the conduction. A technique known as "burn-off", see Chapter 6.5.1.1, was applied to the metallic behaving devices, however it was not always possible to burn-off the metallic CNTs. Nevertheless, with low concentration solutions the CNTs could be burnt-off to show p-type semiconducting behaviour.

Figure 7.9 shows an example of self-assembled HiPCO and laser ablation CNTs current 


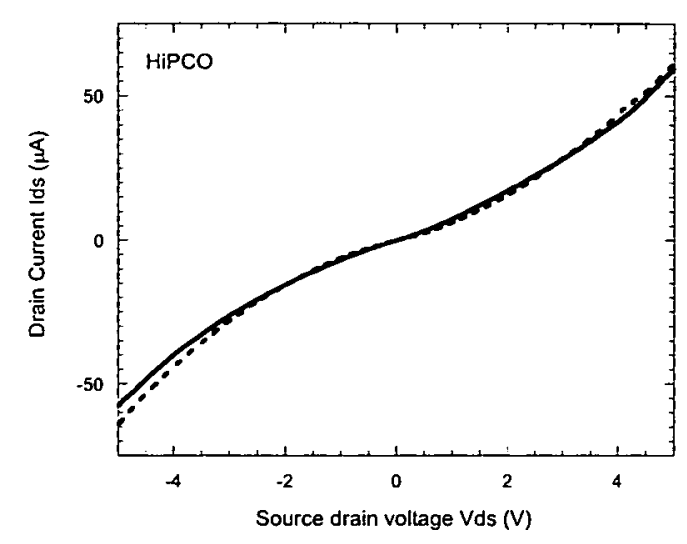

(a) The source-drain characteristics of thiolated and self-assembled HiPCO CNT bundle

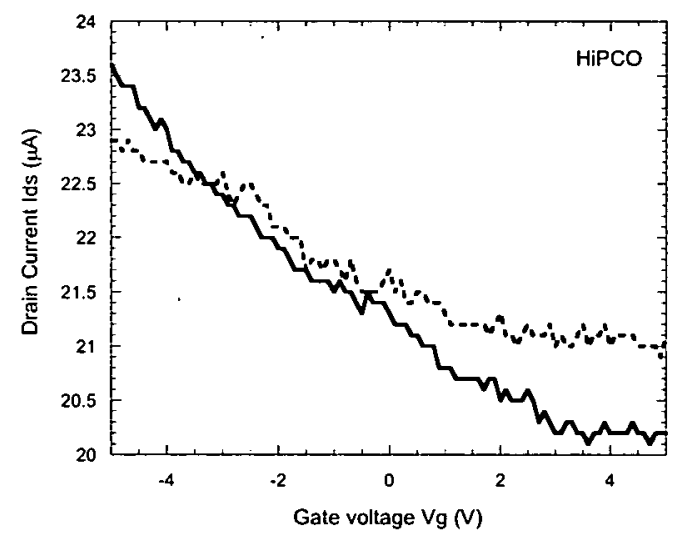

(c) The back gated output characteristics from thiolated and self-assembled HiPCO CNT bundle

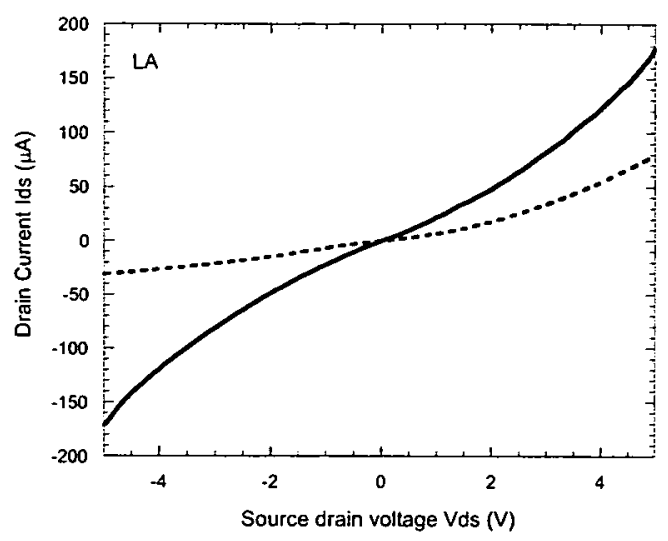

(b) The source-drain characteristics of thiolated and self-assembled laser ablation CNT bundle

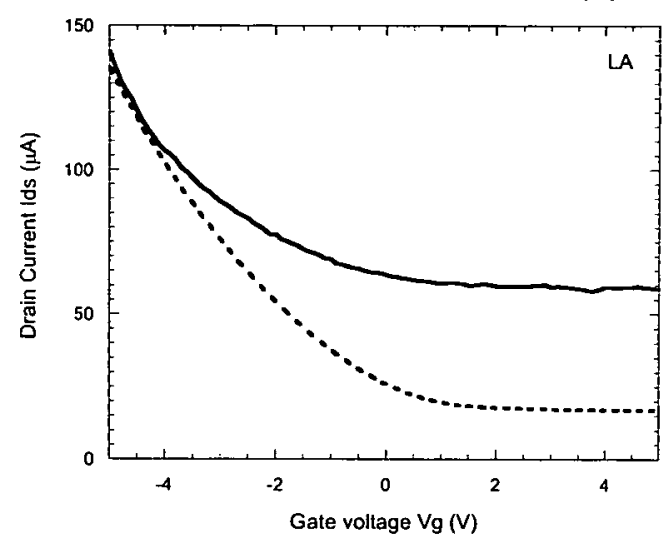

(d) The back gated output characteristics from thiolated and self-assembled laser ablation CNT bundle

Figure 7.9: $I-V$ characteristics of back-gated self-assembled thiolated carbon nanotubes. (a) and (b) show the source-drain characteristics for the self-assembled HiPCO and laser ablation CNTs respectively. (c) and (d) show the backgated output characteristics for the self-assembled HiPCO and laser ablation CNTs. The whole line and the dashed line show two different devices on a single chip.

voltage characteristics. Figures 7.9(a) and 7.9(b) show the source-drain characteristics of the self-assembled CNTs at $\mathrm{Vg}=0 \mathrm{~V}$. Figures 7.9(c) and 7.9(d) show the backgated output characteristics at Vds $=2 \mathrm{~V}$. The gate dependent effects of the HiPCO CNTs appear reduced to those of the laser ablation CNTs. Although the magnitude of the sourcedrain current is of the order of microamps for both types of devices, the HiPCO CNTs appear weakly p-type although largely metallic. The laser ablation CNTs by contrast show a strong metallic component, but a greater indication of p-type semiconducting behaviour. 
The crucial difference between the two types of tube used here is the ability to suspend the laser ablation CNTs being an improvement on HiPCO CNTs. As a practical consideration the HiPCO batch purchased in 2002 displays a powder consistency and a tendency to form tight bundles. The Laser ablation CNTs are more rope like and make a better suspension. However, in these experiments both types of CNTs have bundled together. The working device yield is also very low, less than $10 \%$, presumably due to limitations in the suspensions.

Each device on any given chip made here has had different current voltage behaviour, between stable metallic bundles and small groups of CNTs which are easily burnt off by the application of a source drain voltage. As yet there is no clear indication of how the different chiralities of CNTs present in any given bundle will affect the electrical characteristics of the CNT devices. However, the HiPCO CNTs, which had formed large bundles, show a stronger metallic component than the laser ablation CNTs, which had formed smaller bundles.

At present, it is promising to show that CNT devices have been made by the direct thiolation process without damaging the CNTs so dramatically that the electrical characterisation is impossible. Field-dependent device characteristics have been observed when low concentrations of CNTs bridge electrodes. Otherwise metallic conduction has dominated.

\subsubsection{Electronic Characterisation Summary}

The electronic characterisation of the self-assembled thiolated CNTs has shown that the CNT device vary considerably depending upon the bundle formed. Devices have been observed to behave as metallic conductors and p-type field dependent devices. The suspension of CNTs is thought to be limiting the device uniformity.

\subsection{Conclusion}

The plasma thiolation process has been successful in the production of an environment capable of attaching thiols to CNT sidewalls. The heating of the sulphur and the addition of a $\mathrm{H}_{2}$ feed stock gas were both essential in creating such an environment as 
without either CNTs were not thiolated. The plasma chemistry has also been advantageous for the thiolation without the addition of excess of oxygen contaminants which are known to alter the electrical behaviour of the CNTs. The direct thiolation method used here has been an advantage over the previous thiolation methods [33-35] as no long acid purification steps were required and the resulting structure did not involve any long or short chain molecules between the thiol and the CNTs.

The SEM images have shown that both HiPCO CNTs and laser ablation CNTs can be self assembled across gold electrodes after direct plasma thiolation. The reaction dynamics of the self-assembly process in relation to the chain length of the carbon nanotube appear to have been advantageous in these experiments, allowing the CNTs to bridge Au electrodes over an oxide region. The bridging was in contrast to CNTs self-assembled perpendicular to the Au surface as was the case for the thiolated CNTs made by Liu et al. [34].

Self assembled CNT devices measured in back gated geometry were observed to act as large bundles of metallic CNTs, or as p-type field dependent devices. Although the sulphur plasma has indeed been shown to allow CNT self-assembly across electrodes the yield of working devices is relatively small and unreliable. It is proposed that further improvements to the CNT suspensions and the patterning of $\mathrm{Au}$ microdots between electrode structures could be used to optimise the thiolated CNT self-assembly process.

In the next chapter, a second self-assembly method is proposed whereby CNTs are attracted by $\pi$ bond interaction to a molecule stamped onto a substrate surface. The yield of the working device is found to increase as is the uniformity of the CNT bundles bridging the electrodes. The molecular stamping and self-assembly methods will be discussed along with the electrical characterisation of the devices. 


\section{Chapter 8 Molecular Stamping to Position Carbon Nanotubes}

The need to selectively position carbon nanotubes (CNTs) for CNT device applications has been addressed in the introductory chapters. Direct thiolation of CNTs was developed in earlier work as a means of positioning CNTs and is discussed in Chapter 7. However, the direct thiolation and self-assembly process resulted in a low yield of working CNT devices, largely due to poor suspensions of the CNTs.

A molecular stamping technique has been developed whereby CNTs can self assemble due to $\pi-\pi$ interactions with a pyridine molecule. The molecular stamping and selfassembly techniques have resulted in high device yield of approximately $90 \%$. The fabrication process and the self-assembly for CNTs is discussed in the following chapter, along with the improved suspensions of CNTs for device applications. Finally the selfassembled CNT devices are characterised electronically. The potential applications of this method will be discussed.

\subsection{Molecular Stamping and Self-Assembly Techniques, a Brief Review}

The motivating factor for the work carried out here is the need to position CNTs without interacting with the inherent electronic properties of the CNTs, in a fast, simple high yield process.

Molecular stamping for CNT device fabrication had previously been developed for the positioning of CNTs by Rao et al. published in Nature [38], using polar molecules and surface passivation. The polar molecule used by Rao et al. [38] was 2-mercapto imidazole, the non-polar region 1-octadecanethiol and the passivation region octadecyltrichlorosilane. It has also been seen by other authors that well dispersed CNTs selectively attach to aminopropyltriethoxysilane $[170,171]$ either over entire substrates or 
by selectively exposing areas over an electrode structure. It is therefore interesting to speculate the dominant mechanism in the method proposed by Rao et al. . It could be either the polarity of the stamped regions, the aminosilane layer or the carbon $\pi$ bonds in the imidazole. In addition the fabrication process outlined by Rao et al. was unclear and indicated the requirement of many stages.

To produce CNT devices here, CNTs were self-assembled onto clean silicon dioxide substrates or onto predefined Au electrodes using molecular stamping techniques. A method is proposed using soft-lithography inking techniques $[40,41]$ of 2 -thiolpyridine a non-polar and short chain R-thiol molecule at room temperature, as a two step fabrication process for CNT devices. The selection of 2-thiolpyridine is due to the short chain length as less interaction with the nanotube electronic behaviour is expected. The pyridine end of the 2-thiolpyridine molecule is expected to attract the CNTs by $\pi$ bond interactions, as shown in other work for large pyrenyl functional groups to nanocarbon materials [172].

An advantage to this method over the self-assembly techniques described by others [38, $170,171]$ is the simplicity of the stamping procedure. The method developed here has the potential for easy application for both pristine and functionalised CNTs, with a high working device yield.

\subsection{Molecular Stamping for CNT Devices}

\subsubsection{Molecular Stamping Experimental Procedure}

The device fabrication was attempted with two distinct methods. Methods I and II are shown schematically in figure 8.1 and the fabrication process described by table 8.1.

The stamping mechanism is identical in both cases and can be described by three main stages. 1) The production of a Poly(dimethylsiloxane) (PDMS) stamp, 2) the stamping of 2-thiolpyridine onto the relevant surface and 3) the self-assembly of the CNTs onto the surfaces. These stages are described below in more detail.

1. Production of the PDMS stamp: To produce the PDMS stamp a clean Si wafer was used to fabricate the mask mould. The Si wafer was patterned using e-beam 

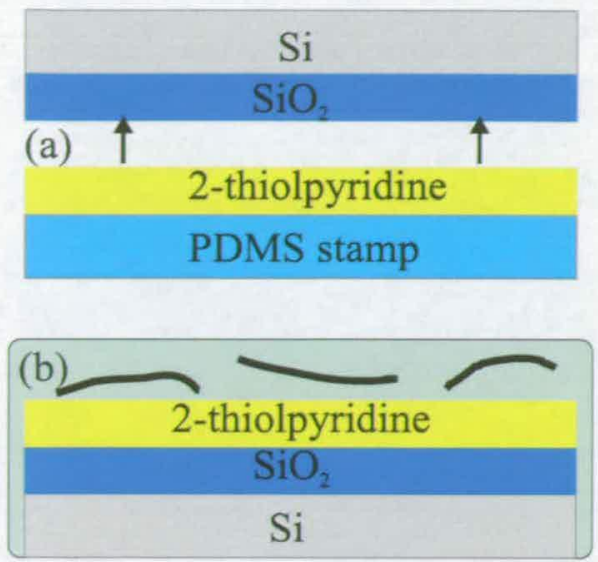

(c)

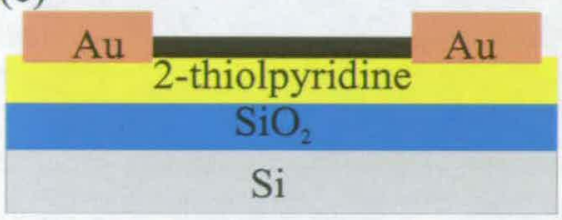

(a) Method I
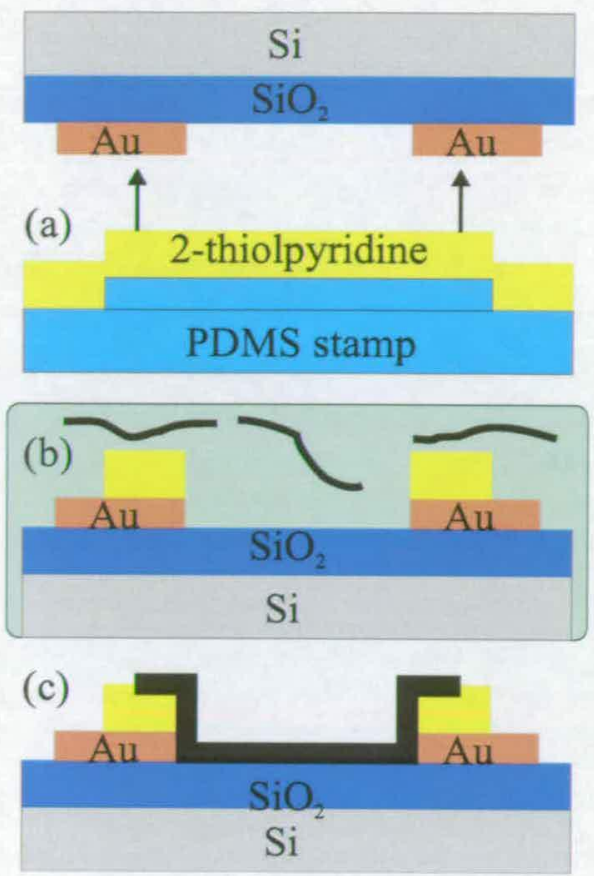

(b) Method II

Figure 8.1: A schematic representation of the molecular stamping process

lithography and the developed patterns etched to a depth of $20 \mu \mathrm{m}$ using TMAH $(25 \%)$ at $90^{\circ} \mathrm{C}$ resulting in smooth sloped sidewalls.

The etched wafer surfaces were then spin coated with a fluorocarbon containing solvent, Optool DSX from Daikin, immediately prior to being spin coated with a 10:1 weight ratio of Sylgard 184 base to curing agent respectively. Prior to spinning, the Sylgard 184 mixture was stirred thoroughly and then degassed in a vacuum chamber for 2 hours or until no air bubbles remained in the mixture.

After curing at $100^{\circ} \mathrm{C}$ for 2 hours, the PDMS stamp could be easily removed from the Si master, with no damage to the stamp and complete transfer of the original pattern.

2. Molecular stamping of 2-thiolpyridine: $0.120 \mathrm{~g}$ of 2-thiolpyridine was dissolved in $30 \mathrm{ml}$ ethanol at room temperature. The PDMS stamps fabricated as in part 1) were placed on a glass slide, with the patterned surface exposed to an oxygen plasma for $30 \mathrm{~s}$, producing a hydrophilic surface. The stamps were then spin coated with the 2-thiolpyridine in ethanol solution, minimising the time between plasma exposure and stamping. 


\begin{tabular}{|l|l|}
\hline Method I & Method II \\
\hline $\begin{array}{l}\text { (a) } \mathrm{SiO}_{2} \text { substrate is stamped with a 2- } \\
\text { thiolpyridine coated unpatterned PDMS } \\
\text { stamp. }\end{array}$ & $\begin{array}{l}\text { (a) Au electrodes are fabricated onto } \\
\mathrm{SiO}_{2} / \mathrm{Si} \text { substrate. The electrodes are } \\
\text { cleaned and heated before being care- } \\
\text { fully aligned and contacted with a 2- } \\
\text { thiolpyridine coated patterned PDMS } \\
\text { stamp. }\end{array}$ \\
\hline $\begin{array}{l}\text { (b) The stamped substrate is submerged } \\
\text { in a solution of CNTs dispersed in 1,2- } \\
\text { dichloroethane. The CNTs self-assemble } \\
\text { over the substrate surface. After 12 hours } \\
\text { the substrate is removed and cleaned in } \\
\text { clean 1,2-dichloroethane. }\end{array}$ & $\begin{array}{l}\text { (b) The stamped substrate is submerged } \\
\text { dichloroethane. The CNTs self-assemble } \\
\text { over the substrate surface for 12 hours. }\end{array}$ \\
\hline $\begin{array}{l}\text { (c) Au electrodes are fabricated on top of } \\
\text { the self-assembled CNTs on the } \mathrm{SiO}_{2} \text { sub- } \\
\text { strate to make the final device. }\end{array}$ & $\begin{array}{l}\text { (c) The substrate is rinsed clean in 1,2- } \\
\text { dichloroethane. Resulting in the final de- } \\
\text { vice. }\end{array}$ \\
\hline
\end{tabular}

Table 8.1: The self-assembly fabrication process

Meanwhile the predefined electrodes in method II were sonicated in clean ethanol and heated at $120^{\circ} \mathrm{C}$ in an oven for 20 minutes to clean the gold. The substrates were held by a vacuum chuck suspended above the PDMS stamps using a modified optical microscope shown in figure 8.2. The vacuum chuck position was controlled in $\mathrm{x}, \mathrm{y}, \mathrm{z}$ and rotation.

The PDMS stamps remained on glass slides and using a backlight geometry microscope, the stamps and the wafer surfaces could be accurately aligned by eye. The substrate was then lowered using the vacuum chuck and carefully brought into contact. The stamps and the wafer surfaces were held in contact for 1 minute before release. Each stamp was used only once.

3. Self-assembly of carbon nanotubes onto the wafer surfaces: The CNTs used in these experiments were grown in house by laser ablation method [169]. To obtain a good dispersion of the CNTs, $10 \mu \mathrm{g}$ of CNTs were sonicated in $10 \mathrm{ml}$ of 1,2 dichloroethane for 30 minutes - 1 hour. Initially small clumps of CNTs could be observed, but after sonication the solution was clear to the naked eye, at this stage sonication was stopped. 
The functionalised substrates or Au electrode surfaces produced by the stamping method outlined were then placed in the CNT solution immediately after stamping of the 2-thiolpyridine in part 2 and left in the solution for a minimum of 12 hours.

For both methods I and II the electrodes were defined by e-beam lithography. The metal was deposited by e-beam evaporation with a $5 \mathrm{~nm}$ Ti sticking layer and 25 $\mathrm{nm}$ of $\mathrm{Au}$, and patterned using standard lift-off techniques as described in the microfabrication chapter. Immediately after removal from the self-assembly solution the substrates were rinsed in clean 1,2-dichloroethane before being characterised further.

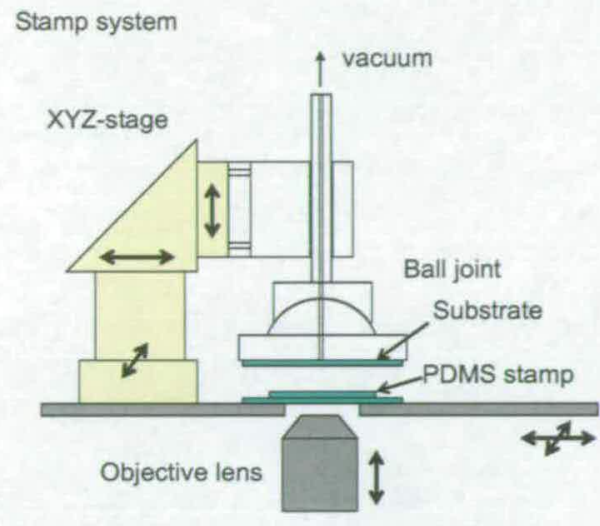

(a) Schematic of the vacuum chuck

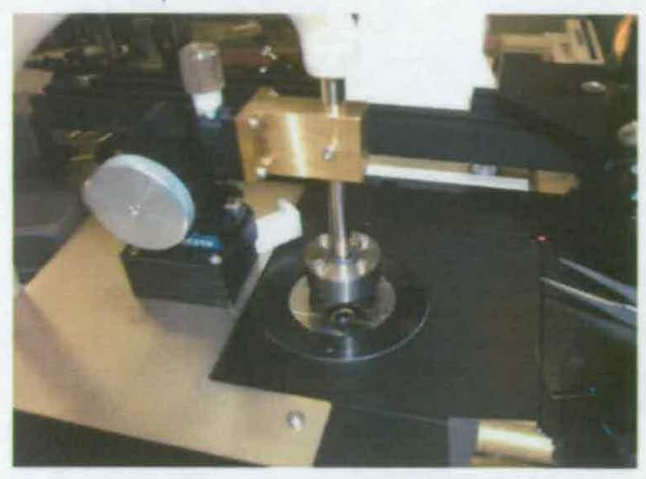

(b) Photograph of the vacuum chuck

Figure 8.2: The vacuum chuck

SEM characterisation of the surfaces was used to show that CNTs had successfully adhered to the substrate surfaces, using the Hitachi S4700 and S4500 SEM. The electronic properties of the devices produced were probed using the HP4156B analyser. All devices were back-gated using a prefabricated gold contact on the backside of the Si-wafers.

\subsubsection{The Molecular Stamping Mechanism}

For the self-assembly of CNTs using 2-thiolpyridine to be successful, it is necessary to consider the mechanism of the self-assembly process. A diagram of the 2-thiolpyridine molecule is shown in figure 8.3.

The attachment of the 2-thiolpyridine to the $\mathrm{SiO}_{2}$ and Au surface is believed to be due 


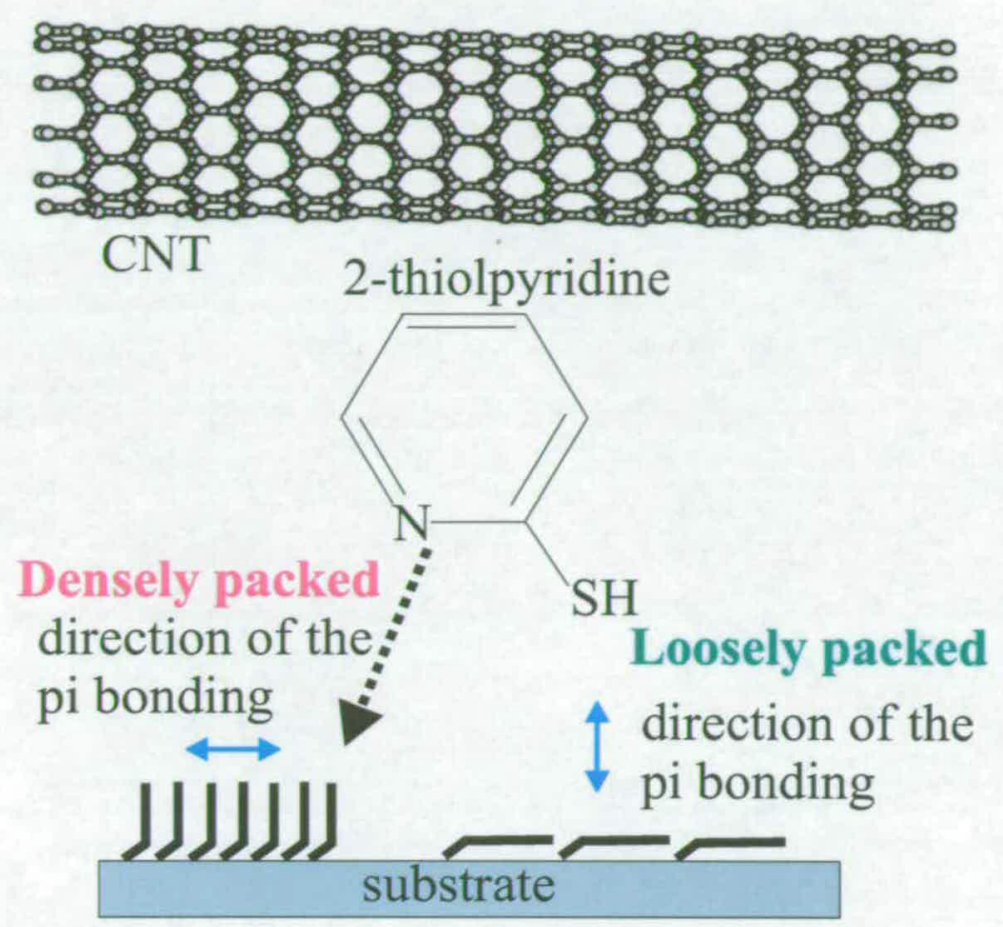

Figure 8.3: A schematic representation of the 2-thiolpyridine and CNT self-assembly process

to the thiol ( $\mathrm{SH}$ ) bond on the end of the 2-thiolpyridine. It is well known that in the case of thiol and gold the R-SH bond easily becomes R-S-Au [40,41] and it is believed that a similar effect will take place between $\mathrm{SH}$ and $\mathrm{SiO}_{2}$ with the dangling bonds on the oxide surface. X-ray photoelectron spectroscopy data collected from a 2-thiolpyridine stamped $\mathrm{SiO}_{2}$ sample has confirmed the presence of sulphur, nitrogen and carbon on the substrate surface ${ }^{1}$. The second stage of the self-assembly process depends on the pi bond interaction between the CNTs and the pyridine end of the 2-thiolpyridine as shown in figure 8.3 .

Several considerations were needed at this stage with regard to the concentration of the 2-thiolpyridine solution. The concentration must be sufficiently dense such that there is an even coating of 2-thiolpyridine over the stamped regions. If the concentration is too dense then the 2-thiolpyridine is believed to close-pack whereby the pi bonds from the pyridine are attracted only to the pi bonds of other pyridine molecules nearby, disallowing self-assembly of the CNTs. By optimising the concentration of the 2-thiolpyridine, self-assembly of CNTs by molecular stamping was achieved.

\footnotetext{
${ }^{1}$ Not shown here.
} 


\subsubsection{PDMS Stamp Geometry: Large Stamps}

Initially stamps were fabricated as $20 \mu \mathrm{m}$ by $120 \mu \mathrm{m}$ rectangles and aligned onto 100 $\mu \mathrm{m}^{2}$ electrodes as shown schematically in figure 8.4 .

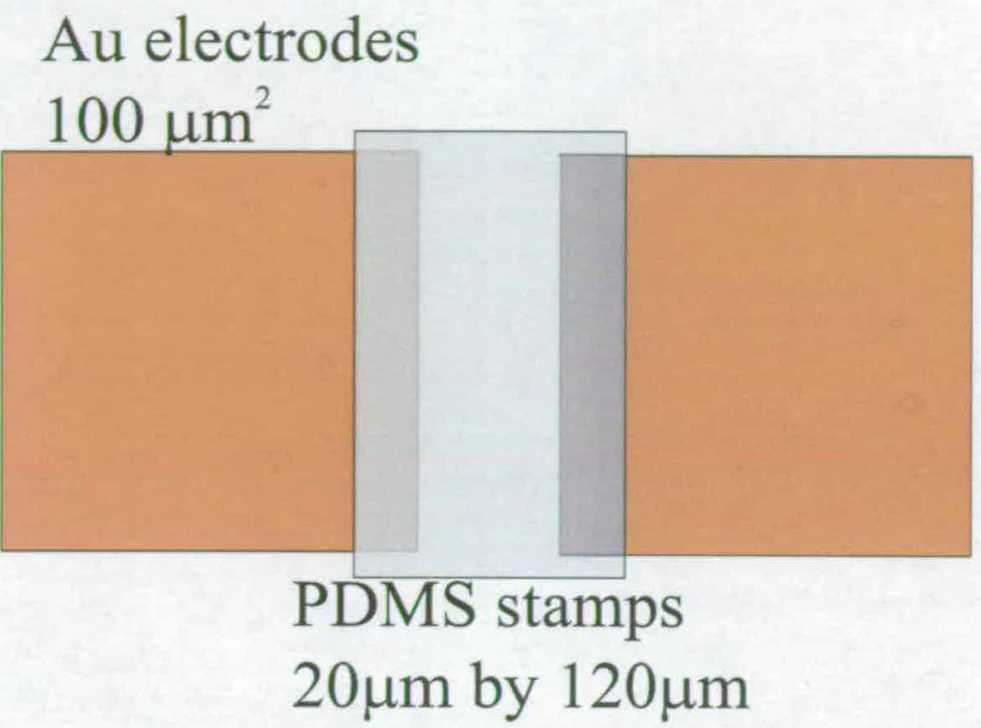

Figure 8.4: A schematic representation of the course alignment stamp and electrode geometry

Shown below in figures 8.5(a), 8.6(a), 8.7(a) are the SEM images of stamped surfaces where CNTs have self-assembled between the electrodes. In figures 8.5(b), 8.6(b), 8.7(b) the corresponding 2-probe source-drain measurements are shown.

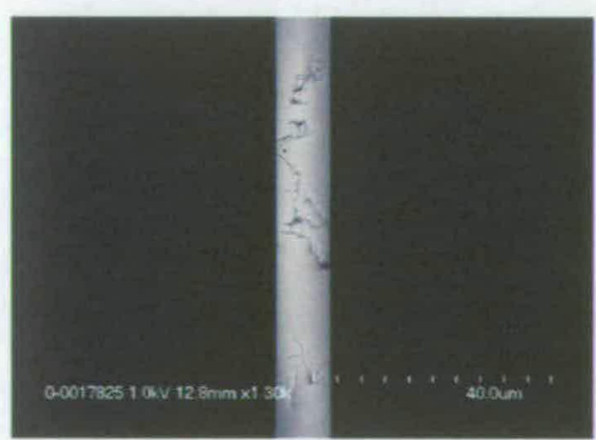

(a) SEM image of the CNTs bundle

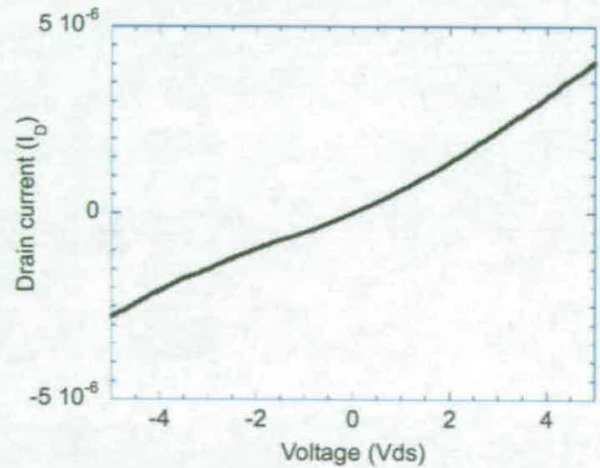

(b) Source-drain characteristics of the bundled CNTs

Figure 8.5: The SEM image of a large bundle of CNTs and the measured source-drain characteristics

It can be seen that in the case of figure 8.5, where a large bundle of CNTs are observed to 
bridge the electrodes, a high current is observed, similar to purely metallic conduction.

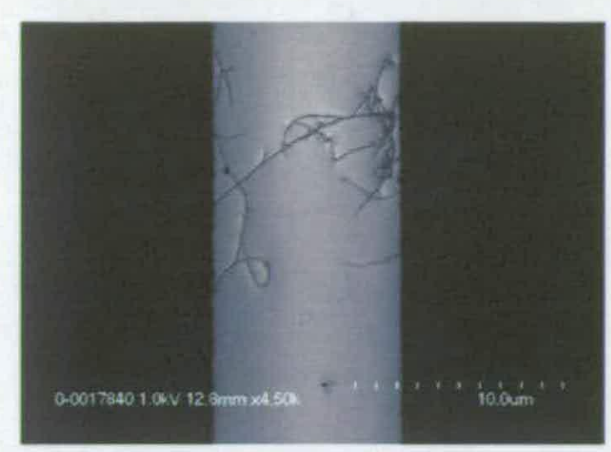

(a) SEM image of a CNTs bundle junction

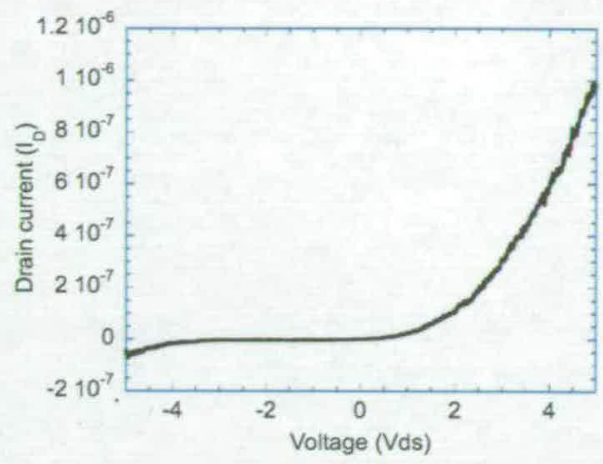

(b) Source-drain characteristics of the CNTs bundle junction

Figure 8.6: SEM image of a bundle of CNTs with a junction. Corresponding source-drain characteristics are shown.

Figure 8.6 shows a bundle of CNTs making a junction with a smaller bundle. The drain current as a function of source drain voltage shows the CNTs to be rectifying in nature.

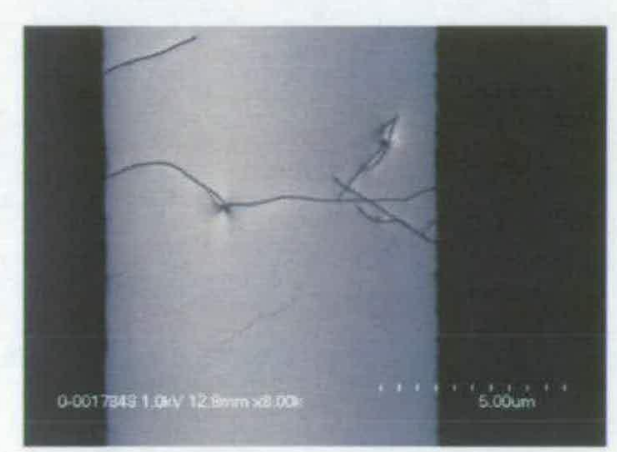

(a) SEM image of a small junction of CNTs

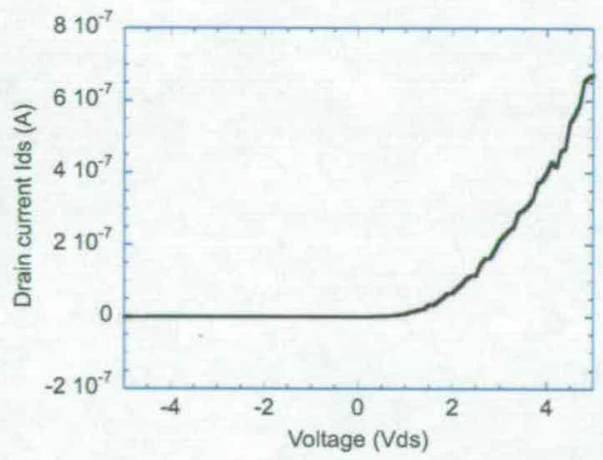

(b) Source-drain characteristics of the CNT junction

Figure 8.7: The I-V and SEM image of a small bundle of CNTs clearly showing a junction

A smaller CNT junction, presumed to be only several tubes or even a single CNT, imaged due to the high contrast between the conducting tubes and the insulating oxide, is shown in figure 8.7. The drain current as a function of source drain voltage clearly shows rectifying behaviour similar to the previous junction characteristics. Here, where each individual CNT devices have been imaged and probed individually, the dominance of the metallic CNTs in large bundles is observed. Small CNT bundles have shown increased semiconducting behaviour here, with asymmetrical source-drain characteristics. It would also be possible to observe small bundles of metallic CNTs, however, 
here semiconducting device behaviour was shown. These characteristics confirm the earlier discussion in Chapters 6 and 7, where the CNT device behaviour varied due to statistical differences in the bundles.

Although the stamping method using large PDMS stamps has been shown to produce a region where CNTs can self assemble between pre-defined electrode structures, the working device yield is low, at approximately $30 \%$. Primarily the low yield is thought to be due to the large gap between the electrodes of $10 \mu \mathrm{m}$.

\subsubsection{PDMS Stamp Geometry: Small Stamps}

To counteract the low device yield achieved when the electrode spacing is large, a new stamp and electrode structure was created by e-beam lithography and is shown in figure 8.8. The new structure allowed spacing between electrodes of $100 \mathrm{~nm}, 300 \mathrm{~nm}$, $500 \mathrm{~nm}$ and $1 \mu \mathrm{m}$ and stamp structures of $30 \mu \mathrm{m}$ by $5 \mu \mathrm{m}$.

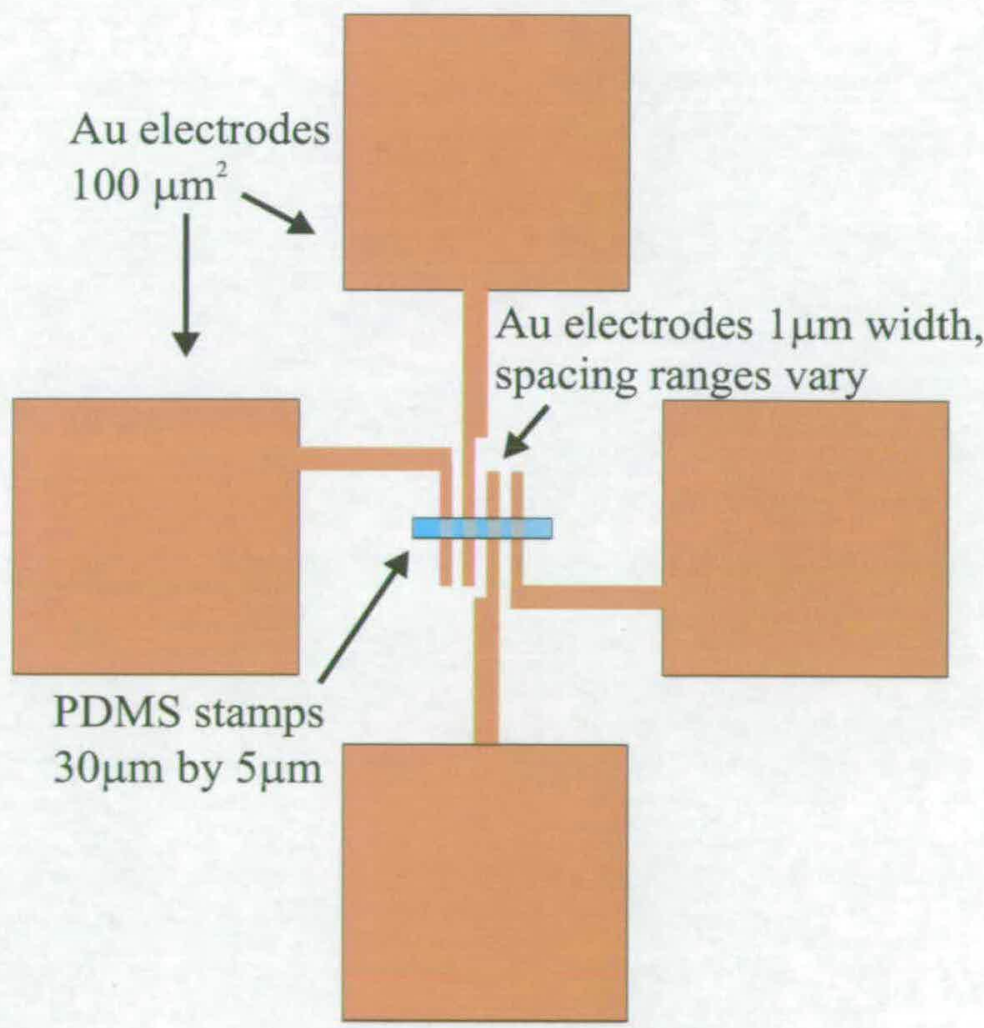

Figure 8.8: A schematic representation of the fine alignment and electrode geometry

Following method I as described in Section 8.2, figure 8.1 and table 8.1 the CNTs were 
often found to be covering the $\mathrm{SiO}_{2}$ substrate surface, appearing as a uniform coating. After the fabrication of the electrodes by e-beam lithography and e-beam metal evaporation, CNTs were observed to be bridging the electrodes structures. A success rate of $90 \%$ was found, success being defined as CNTs bridging the electrodes to allow current flow. Figure 8.9 shows a typical surface of a sample fabricated via method I.

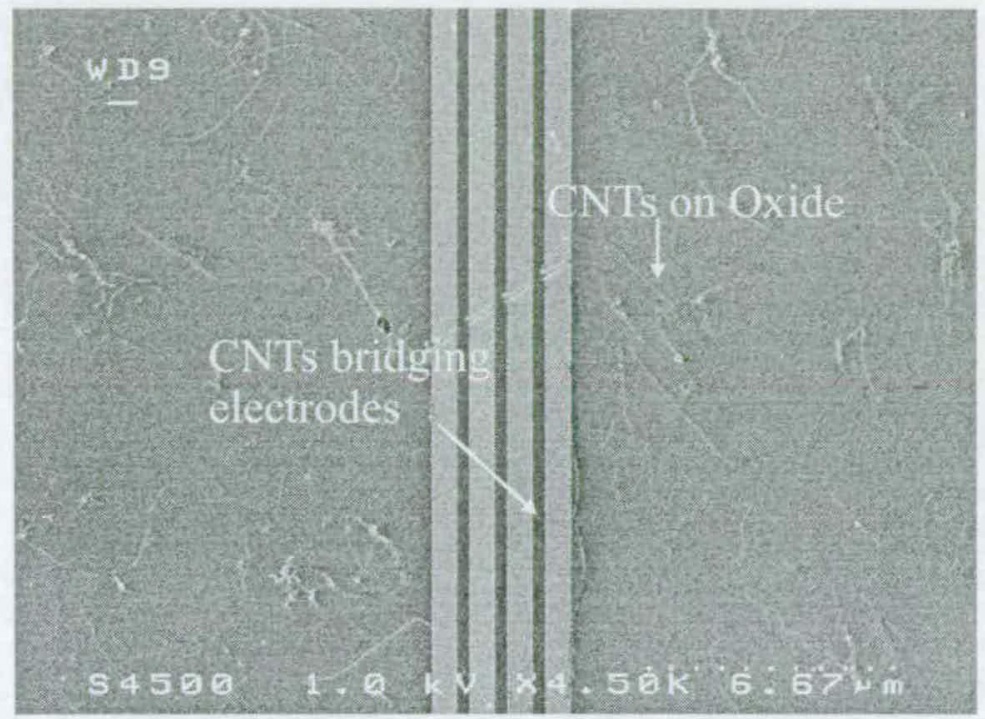

Figure 8.9: SEM image of CNT device made via method I

Similar geometry electrodes to those made in method I were fabricated and following method II CNT devices were produced. Method II also showed a high yield of successful conduction between electrodes, with approximately $90 \%$ of contacts probed showing conduction. Figure 8.10 shows the typical surface for a device made by method II using the small geometry stamp system.

It should be noted that due to inaccuracies of the manual stamping method, the stamp often deformed and 2-thiolpyridine was stamped across the substrate surfaces, not just onto the electrode regions during fabrication via method II. Yet using method II without alignment the device yield is lower, at around 30\%. CNTs were not observed over the substrate surface for the coarse alignment geometry used with method II stamping technique as the stamp area was larger and therefore more rigid. It would require greater pressure to deform the stamp. An oxygen plasma removal process could be applied to remove the excess CNTs not bridging the electrodes.

The self-assembly method developed here is different from those produced by Rao et 


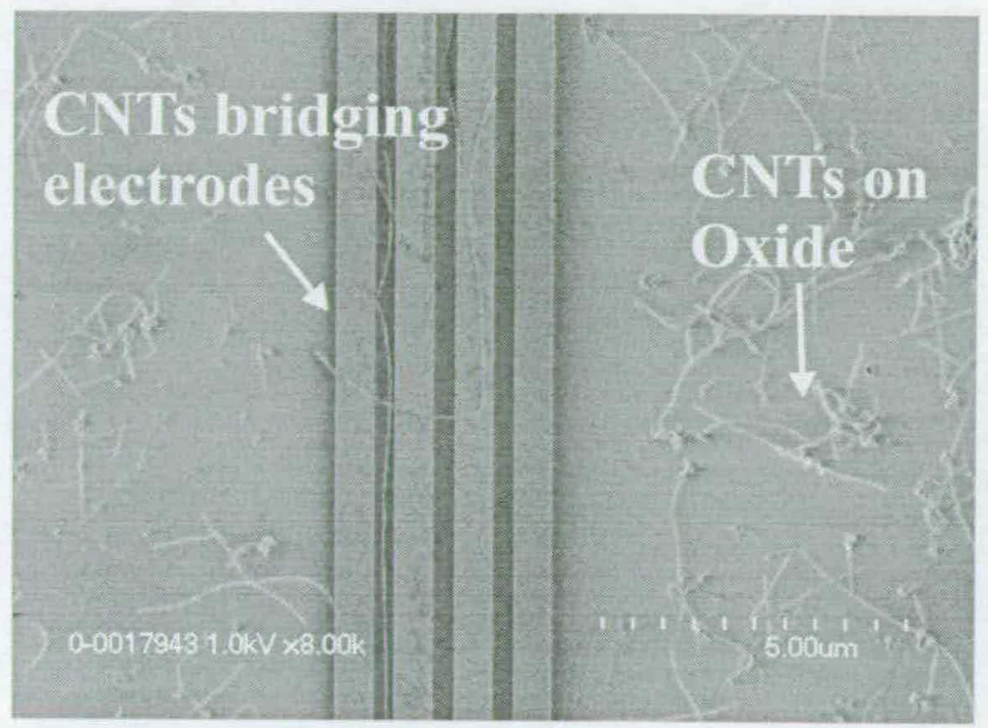

Figure 8.10: SEM image of CNT device made via method II

al., Lay et al. and Lewenstein et al $[38,170,171]$ where aminosilane layers have been used. In the case of the aminosilane self-assembly, CNTs tended to form more isolated groups and have not been as bundled as the devices made here, presumably due to initial suspension improvements. The Lay and Lewenstein methods both required stages to remove excess CNTs on the substrates. However, the self-assembly method developed here has the advantage of being a fast and simple fabrication process. The PDMS stamps are easily made and the 2-thiolpyridine self-assembles well across a substrate. Also, the method can be readily applied to both pristine and low stoichiometry functionalised CNTs.

\subsubsection{Molecular Stamping Summary}

The stamping of 2-thiolpyridine over $\mathrm{SiO}_{2}$ substrates and onto predefined electrode structures has been a useful method for the self-assembly of CNTs. A low concentration of 2-thiolpyridine in ethanol has been optimised to allow a dense layer of the 2thiolpyridine without close packing of the pyridine molecules. By suspending the CNTs in 1,2-dichloroethane at low concentration, the $\pi$ bonds of the CNTs are attracted to the $\pi$ bonds of the pyridine end of the 2-thiolpyridine. Thus allowing for self-assembly of the CNTs. The distance between the electrodes must also be optimised to allow for CNTs to successfully bridge the electrodes. By reducing the electrode spacing the yield 
of working devices was increased:

\subsection{Electronic Characterisation}

The CNT devices fabricated by molecular stamping have all resulted in backgated CNT devices suitable for transistor type behaviour. The electrodes are tested as described in Chapter 3.3, with a source, drain and back gate all probed using the HP4156B analyser.

\subsubsection{Source-Drain Characteristics of Self-Assembled CNTs}

An average example of the source-drain characteristics of devices produced by method I, with a $500 \mathrm{~nm}$ gap between the source and drain electrodes, are shown in figure 8.11(a) 8.11(b), 8.11(c). The differences between each device is the concentration of CNTs in the 1,2-dichloroethane solution. Although carefully measured with $10 \mu \mathrm{g}$ of CNTs in $10 \mathrm{ml}$ of 1,2-dichloroethane, there were slight differences between each solution, resulting in more CNTs on the surface for device method I (a), and decreasing to device method I (c), where the CNT solution was deliberately further diluted during the self-assembly stage.

Figure 8.11(d) shows the typical source-drain behaviour of a device produced by method II, also with $500 \mathrm{~nm}$ gap between the source and drain electrodes. It is seen that the value for Ids is of the same order of magnitude for the devices made by method I (a) and method II. Similarly the magnitude of Ids is similar for devices by method I (b) and (c).

The devices produced by method I (a) (b) and (c), show source-drain curves that are asymmetric around the origin, implying that the CNT devices are semiconducting in nature. There is a reduction in Ids when the gate voltage is altered from $-5 \mathrm{~V},-2 \mathrm{~V}$ to 2 $\mathrm{V}$ and $5 \mathrm{~V}$ during the source drain voltage sweep, figure 8.11, such behaviour observed here is indicative of p-type CNT devices as described in the background section in Chapter 3. The devices produced by method II in contrast have shown no field-dependent behaviour. When the gate voltage is altered the source-drain characteristics follow an identical curve, regardless of whether $\mathrm{Vg}$ is at $-5 \mathrm{~V}$ or $+5 \mathrm{~V}$, or any value in between. It can therefore be stated that method I type devices have shown semiconducting be- 


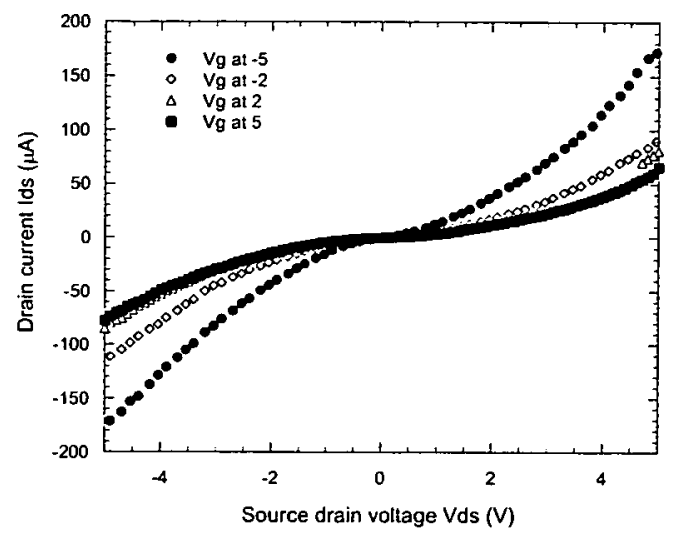

(a) The source-drain characteristics of device Method I (a) at $\mathrm{Vg}=-5 .-2,2$ and $5 \mathrm{~V}$

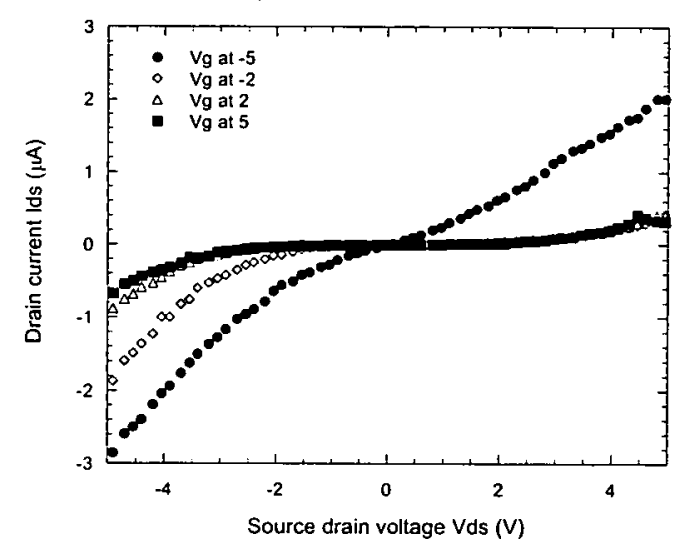

(c) The source-drain characteristics of device Method I (c) at Vg $=-5 .-2,2$ and $5 \mathrm{~V}$

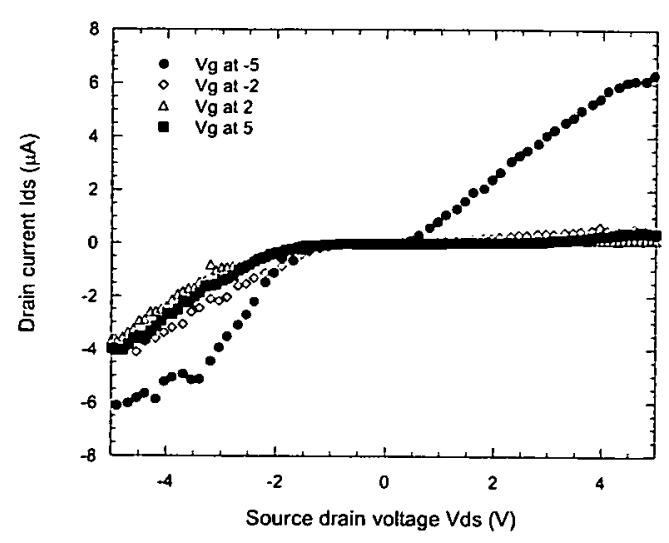

(b) The source-drain characteristics of device Method $\mathrm{I}(\mathrm{b})$ at $\mathrm{Vg}=-5 .-2,2$ and $5 \mathrm{~V}$

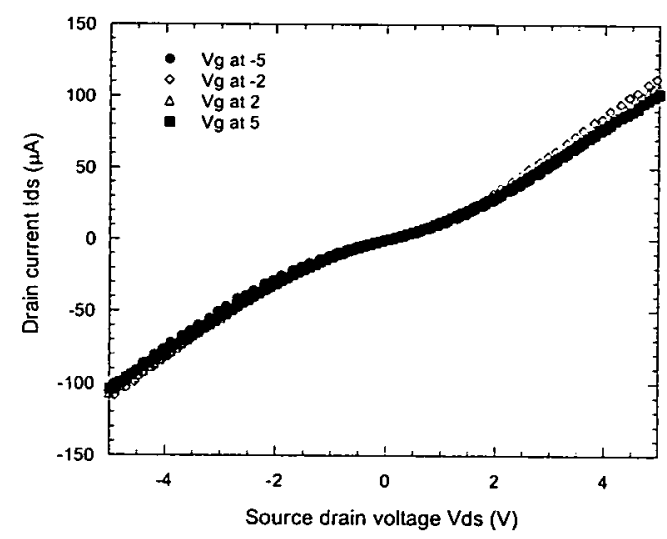

(d) The source-drain characteristics of device Method II at $\mathrm{Vg}=-5 .-2,2$ and $5 \mathrm{~V}$

Figure 8.11: The source-drain characteristics for devices produced by method I and II at $V g=-5,-2,2$ and $5 \mathrm{~V}$. Method I type devices have all shown semiconducting behaviour, with a change in $\mathrm{Vg}$ affecting the drain current during the sourcedrain sweep. At positive Vg, the drain current is reduced. The method II type device shows no dependence on $\mathrm{Vg}$ and acts as a metallic conductor.

haviour whilst method II type devices are metallic.

\subsubsection{Gate Field Dependent Characteristics of Self-Assembled CNTs}

The backgated output characteristics of the devices made by method I and method II are shown in figure 8.12, confirming the p-type behaviour of the field dependent devices. It is noted that method I produces devices with field dependent behaviour characteristic of semiconducting CNTs. Method II has been shown to produce metallic type devices. All field dependent devices have exhibited p-type behaviour as expected 
from unfunctionalised CNTs measured in air.

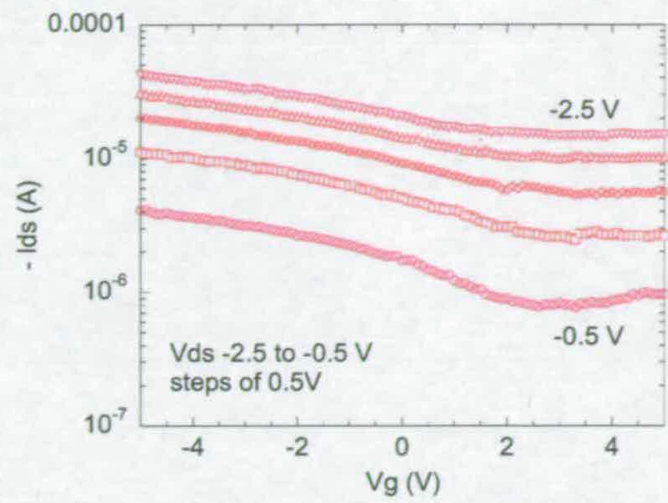

(a) The gate field dependent characteristics of device Method I (a).

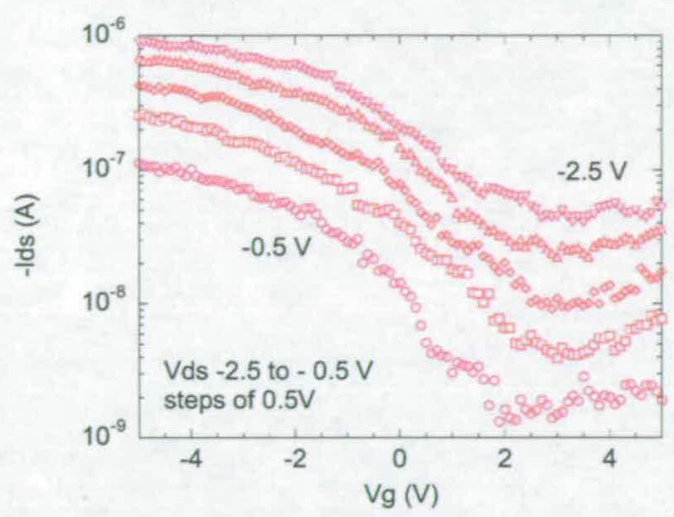

(c) The gate field dependent characteristics of device Method I (c).

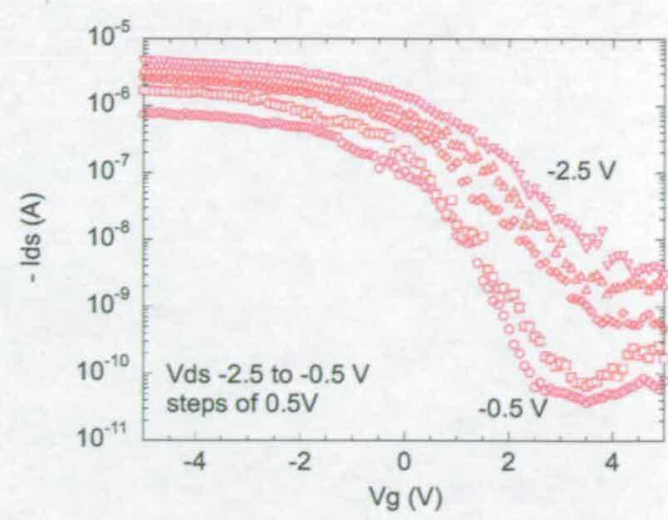

(b) The gate field dependent characteristics of device Method I (b).

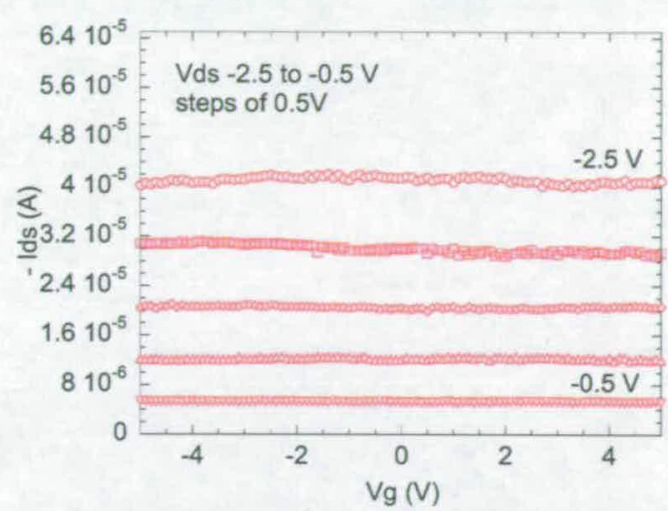

(d) The gate field dependent characteristics of device Method II.

Figure 8.12: The backgated output characteristics for devices produced by method I and II. Vds is altered from $-0.5 \mathrm{~V}$ to $-2.5 \mathrm{~V}$ at $0.5 \mathrm{~V}$ step size. The method I type devices show p-type semiconducting behaviour, with devices (a) and (c) both showing a metallic leakage path. The method II type device shows metallic behaviour.

The devices made by method I (a) show multi-channel CNT FET characteristics, with a relatively high off current shown as $2 \times 10^{-5} \mathrm{~A}$ at $\mathrm{Vds}=-2.5 \mathrm{~V}$. The remaining on current may be due to a metallic CNT component bridging the electrode structure. No burnoff was attempted here. The device made by method I (b) shows the most promising transistor behaviour with a low off current at magnitude of $n A$ and little indication of a metallic leakage current. The device made by method I (c) also shows a small metallic leakage current.

The devices produced in method I behave similarly to other FETs produced by other 
groups having Au electrodes fabricated on top of CNTs over the gate voltage ranges used here $[36,60,173,174]$ and similar oxide thickness. The devices have not yet reached saturation, due to the thickness of the gate oxide. Thinner oxides have been shown to produce devices with larger dependence of Ids at low gate voltages [58,64;68,69, 73], where saturation of the devices was observed within the gate voltage ranges tested here.

As the channel length of the devices is short, $500 \mathrm{~nm}$ for the results shown it is also not possible to reliably determine the mobility of the carriers in the CNT devices, as the channel in such a short channel device will not dominate the carrier flow $[68,73]$, discussed previously in Chapter 3.

It is proposed that the observed field dependent behaviour is due to the effect of the gate voltage on the CNT/electrode interface in each situation, at the channel lengths probed here $(500 \mathrm{~nm})$, the conduction in the CNT FETs is predicted to be due to the field-induced lowering of the Schottky barriers. Due to the oxide being thick, $50 \mathrm{~nm}$, screening of the Schottky barriers at the gate voltages probed has occurred and these devices have not yet reached saturation.

\subsection{Comparison of Field-Dependent Behaviour between Met- hod I and Method II Type Devices}

Returning to the two device fabrication methods, of method I and method II, the fabrication process has resulted predominantly in devices which are field-dependent for method I, and metallic for method II. As all materials used were the same and the microfabrication processes are well characterised, the explanation of such behaviour must lie in the device structure differences due to the altered order of the fabrication processes between method I and method II.

\subsubsection{The Device Structure}

The final device structures for method I and method II are shown in figure 8.13. During the electrical characterisation employed here, no selectivity in the growth of CNTs between metallic and semiconducting was attempted, nor any burn off of metallic CNTs 
attempted as in other work $[60,77]$. Yet, method I type devices are field dependent and method II type devices are not.

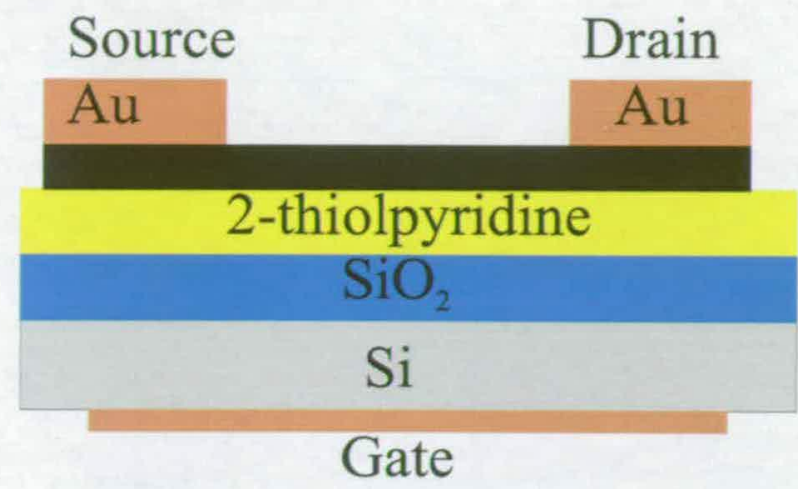

(a) The device structure for Method I type devices

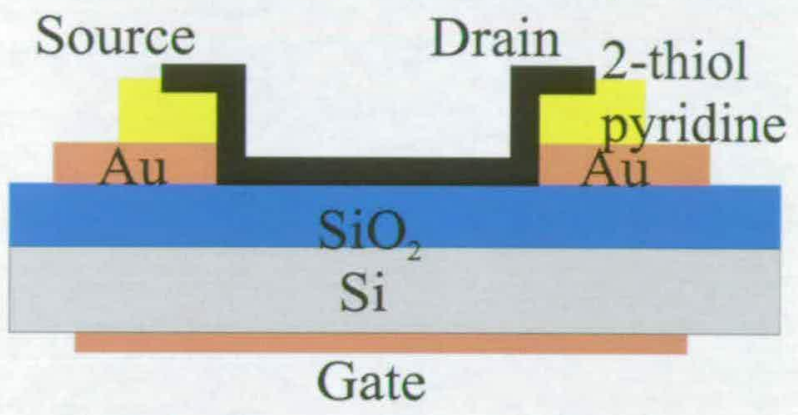

(b) The device structure for Method II type devices

Figure 8.13: The device structures

For method I the device structure is $\mathrm{CNT} / \mathrm{Ti} / \mathrm{Au}$, with any 2-thiolpyridine contribution being beneath the CNT, figure 8.13(a). It is well known that the CNT/Ti contact could have formed titanium carbide which has often resulted in Schottky barrier dependent CNT FET devices $[68,69,73,173]$. It is probable that in the method I fabrication process the CNT and electrode make a good contact, as the Ti is e-beam deposited directly on top of the CNTs, resulting in the field dependent behaviour observed here.

For devices produced by method II the structure is CNT/2-thiolpyridine/Au/Ti, figure 8.13 (b). The quality of the Au/CNT contact is not expected to be as high as the $\mathrm{Ti} / \mathrm{CNT}$ contact made in method I as the 2-thiolpyridine lies between the electrode and the CNT. It is possible in the case of method II devices that the 2-thiolpyridine is acting as a further screening region from the gate field, resulting in the metallic behaviour measured. 


\subsubsection{Gate Screening Effects}

During the method II fabrication process, the CNTs are self-assembled on top of the 2-thiolpyridine layer above the electrode structure. As a consequence of this, there is an extra physical barrier between the CNT and the electrode, as well as between the CNT and the gate field.

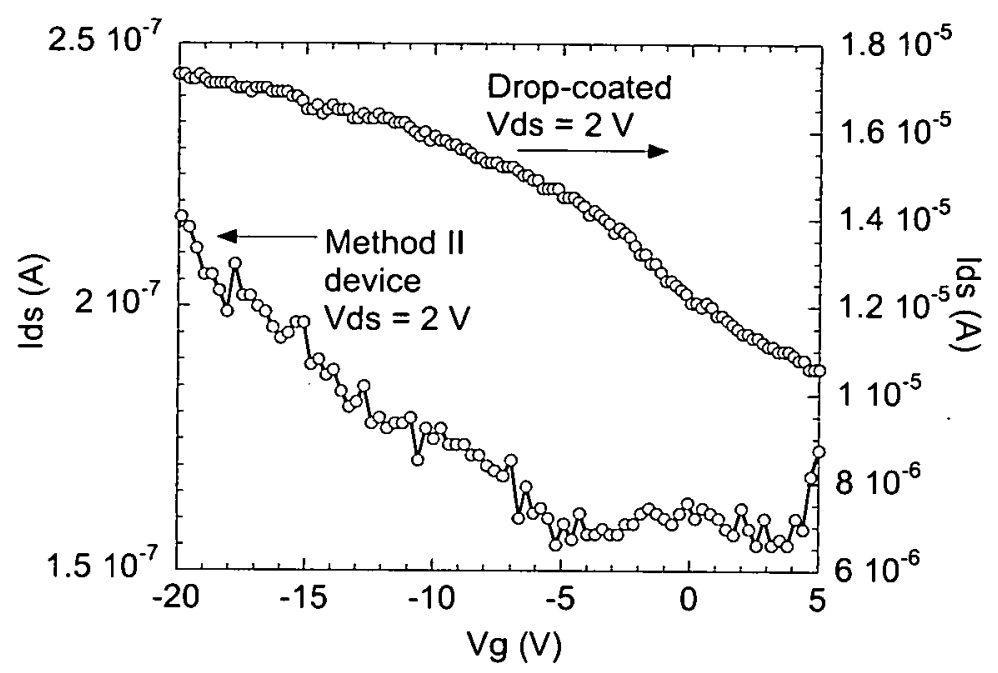

Figure 8.14: The gate dependence of a device made by method II in comparison to a device made by drop-coating of CNTs

Figure 8.14 shows a comparison between the backgated characteristics of a device made by method II, and a device made by drop coating a CNT solution over similar geometry electrodes. The overall conduction for the drop coated device is higher as a consequence of the altered CNT suspension.

Within the gate voltage range of -5 to $+5 \mathrm{Vg}$, the method Il type device shows no field dependent behaviour, figure 8.14. The drop coated devices in contrast shows a weak gate field dependence. By extending the range of the gate voltage in the negative direction to $-20 \mathrm{Vg}$, field dependent conduction was observed in a typical method II device. This confirmed that the physical distance between the gate field and the CNT/electrode interface and the 2-thiolpyridine layer had previously limited field dependent conduction. For further device fabrication such gate screening effects must be considered. 


\subsection{Characterisation of Method I Field Dependent Devices}

\subsubsection{Subthreshold Characteristics}

As discussed in Section 8.3.2, the devices produced by method I are observed to show field dependent behaviour. The devices are considered to be within the subthreshold regime as no saturation has yet been observed, a closer look at the gate dependent current of the devices in the subthreshold regime is shown below in figure 8.15(a) and a logarithmic plot in figure 8.15(b). The method II type devices are shown for comparison, however, as the method II type device shows no dependence on $\mathrm{Vg}$ within the range -5 to $+5 \mathrm{~V}$, they will not be discussed.

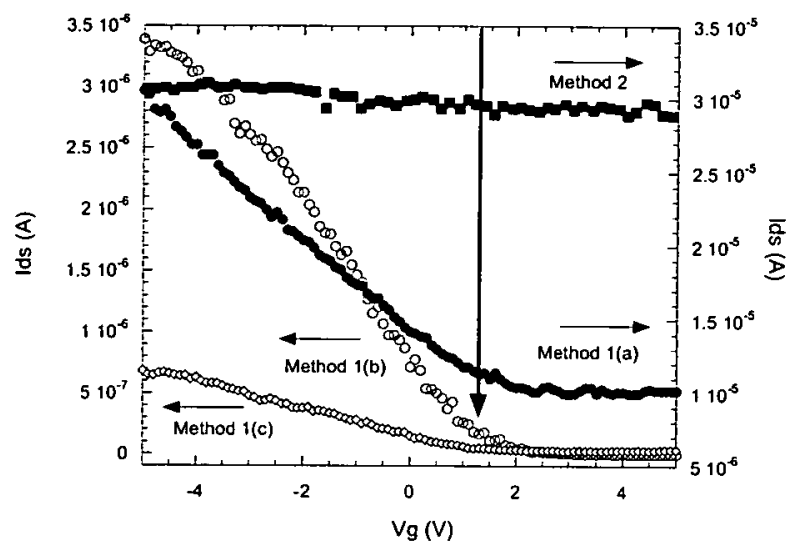

(a) Drain current versus gate voltage at $\mathrm{Vds}=-2 \mathrm{~V}$

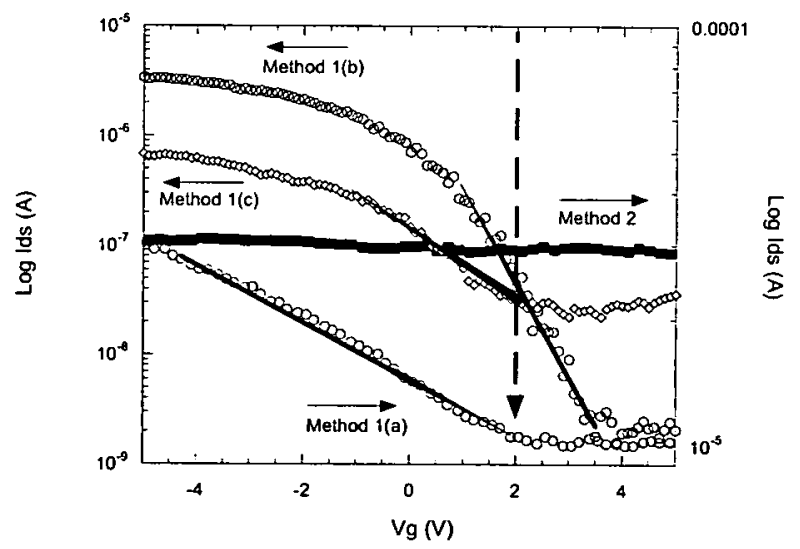

(b) Logarithmic drain current versus gate voltage at $\mathrm{Vds}=$ $-2 \mathrm{~V}$

Figure 8.15: The drain current (Ids) as a function of gate voltage for devices method I (a), method I (b), method I (c) and method II.

The dominance of the Schottky barrier in the CNT-FETs conduction has been studied by 
the dependence of the subthreshold slope on Ids by other authors $[36,68,73]$.

$$
S=\frac{d V g}{d(\log I d s)}
$$

The position of the slope is indicated in figure 8.15(b), on the source-drain current as shown in figure 8.16(d) where it is noted that the difference in Ids over the swept Vg is highest for device method I (b), covering 3 orders of magnitude, whereas method I (a) and (c) each cover only 1 order of magnitude. Both devices method I (a) and (c) each retain a higher off current than the method I (b) device indicating the presence of metallic CNTs for devices (a) and (c).

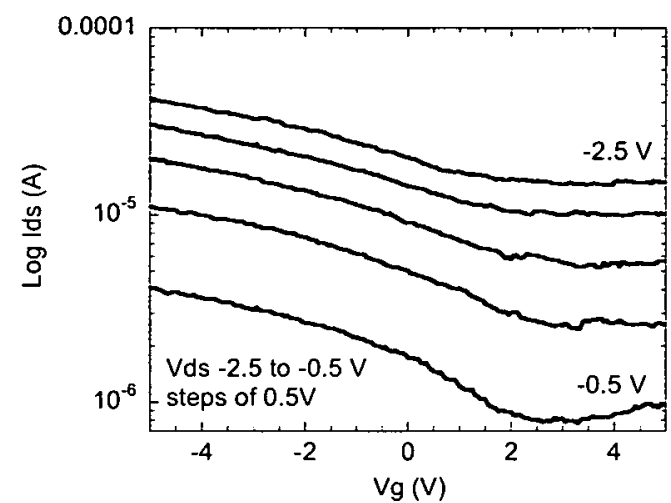

(a) Logarithmic drain current versus gate voltage of device Method I (a)

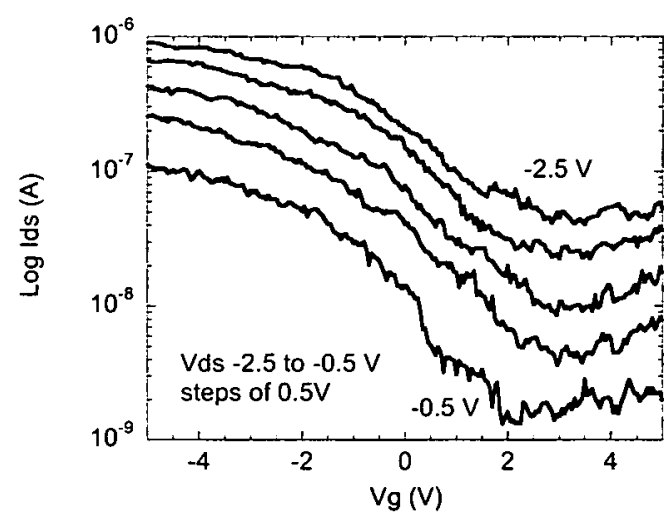

(c) Logarithmic drain current versus gate voltage of device Method I (c)

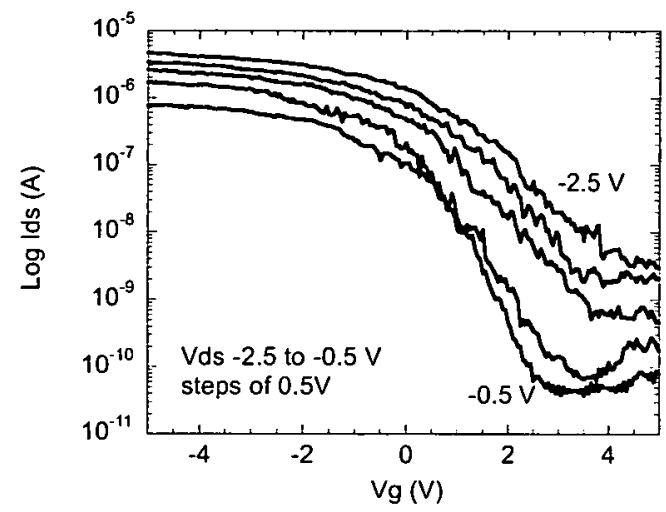

(b) Logarithmic drain current versus gate voltage of device Method I (b)

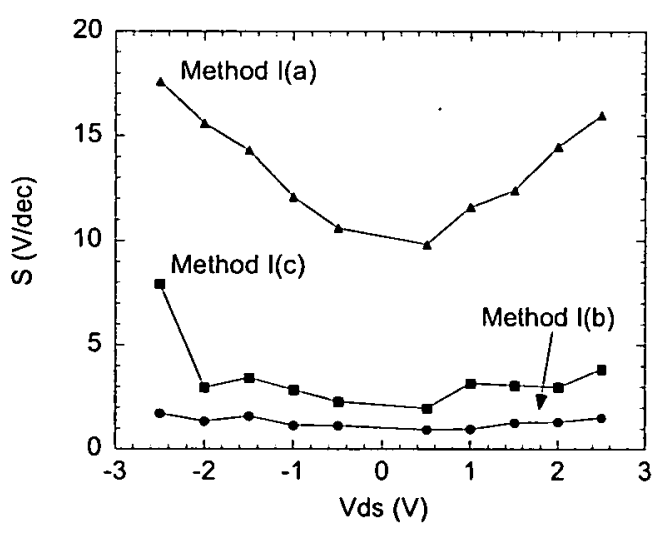

(d) The subthreshold slopes as a function of Vds

Figure 8.16: Logarithmic backgated output characteristics for devices produced by method $I$ and the corresponding subthreshold slope dependence on Vds

Figure 8.16 shows the Ids of the field dependent devices at Vds from $-0.5 \mathrm{~V}$ to -2.5 $\mathrm{V}$. The resulting subthreshold slopes of each device was then calculated and is shown 
as a function of Vds in figure 8.16(d). The device fabricated by method I (a) has shown the greatest dependence on Vds, whilst device method I (b) has shown the lowest. The device produced by method II shows no change as discussed for metallic type conduction. The magnitude of $S$ for device method I (a) is comparable to other work [36] for similar behaving devices.

However, it is noted here that the subthreshold slope may not be a valid analysis for these devices as the inclusion of metallic CNTs can not be ruled out. We therefore are calculating the subthreshold slope for a device with a very large leakage current. Since the CNT dispersions were produced using CNTs fabricated in the same batch, the dominant factor for the differing behaviour between the devices is due to the original suspension of the CNTs in the self-assembly stages.

\subsubsection{Electronic Characterisation Summary}

The molecular stamping and self-assembly methods used here have successfully produced CNT devices. The device reproducibility and behaviour is seen to depend on the initial CNT suspensions. For any given suspension, CNT films were observed to be similar and Ids did not vary at higher Vg. However, the ratio of metallic and semiconducting CNTs could not be homogenised, and as such dramatically altered the overall device characteristics.

By reducing the physical distance between the CNT/electrode interface in method I and having good contact with the CNT and the the electrode, field dependent devices were made, over the gate field range $\mathrm{Vg}-5 \mathrm{~V}$ to $+5 \mathrm{~V}$. By stamping over predefined electrodes, where an increased physical distance between the CNT/electrode interface existed, method II, devices were observed to act as metallic conductors with little or no gate dependence, over the gate field range $\mathrm{Vg}-5 \mathrm{~V}$ to $+5 \mathrm{~V}$. By increasing the gate voltage to $-20 \mathrm{~V}$, p-type field dependence was observed for method II type devices.

\subsection{Statistical Differences in the CNT Devices}

As discussed previously in Chapters 6.5 and 7.4, problems exist in the CNT devices, with bundling of the CNTs causing a large shift in the drain currents observed for any 
given device. In addition, there is also a difference between the chirality of the CNTs in any given bundle. Large variations in the magnitude of the drain current were observed for the fluorinated and further amine functionalised CNT devices in Chapter 6.5. Such problems also existed for the self-assembled thiolated CNTs in Chapter 7.4.

To consider the suspension problems for the CNT devices made by the molecular stamping methods, four devices from the method I (b) chip are shown in Figure 8.17. Device 1 and 2 look similar to each other, both exhibiting semiconducting device behaviour with little indication of a metallic component due to the low off current, at magnitude of nano amps. Device 3 however, is markedly different from the devices shown before as it begins to exhibit ambipolar type behaviour. Such ambipolar behaviour is not impossible for CNT devices in air, although usually higher gate voltages are required.

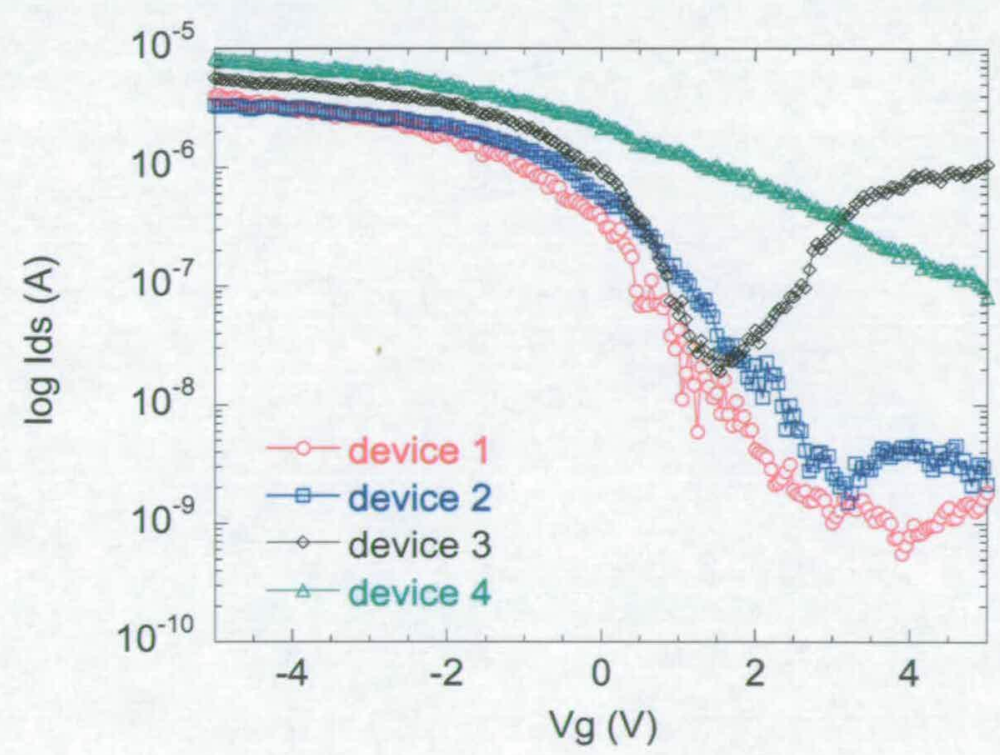

Figure 8.17: Gate field dependent conduction of four devices made by method I (b). Logarithmic backgated output characteristics at Vds $=-2 \mathrm{~V}$

As discussed in the introductory chapters, the p-type conduction observed for CNTs in air is not due to dopants, but rather it is due to the interface between the CNTs and the electrodes. Therefore for device 3 the interface must allow the conduction of holes and electrons depending on the field applied. Finally, device 4 is predominantly metallic. Although a weak field dependence has been observed, this device is clearly not purely semiconducting.

Although the devices produced on the method I (b) sample have shown altered gate 
dependent behaviour, it is interesting to note that the magnitude of Ids at $-5 \mathrm{Vg}$ is approximately the same for each device, in contrast to devices made in Chapters 6.5 and 7.4. As such this is further indication of the uniformity of the CNTs coverage self-assembled over the 2-thiolpyridine in the molecular stamping fabrication process. However, the remaining irregularities in the suspensions of the CNTs between metallic and semiconducting CNTs is the limiting factor to the device reproducibility here. It would therefore be invalid to continue characterisation techniques such as mobility and subthreshold behaviour of the CNTs as the metallic leakage coefficient is too large to ignore in many of the CNT devices made by the molecular stamping methods. Also, the channel length, $500 \mathrm{~nm}$, being too short to make mobility dominance likely [53].

\subsection{Conclusion}

In conclusion the fabrication process outlined has continually allowed successful contact of the CNTs between 2 electrodes. The stamping of 2-thiolpyridine over $\mathrm{SiO}_{2}$ substrates and onto predefined electrode structures has been a useful method for the self-assembly of CNTs, with high working device yield of $90 \%$. A low concentration of 2-thiolpyridine in ethanol has been the optimised to allow a dense layer of the 2thiolpyridine without close packing of the pyridine molecules. By suspending the CNTs in 1,2-dichloroethane at low concentration, the $\pi$ bonds of the CNTs are attracted to the $\pi$ bonds of the pyridine end of the 2-thiolpyridine. Thus allowing for self-assembly of the CNTs. The distance between the electrodes must also be optimised to allow for CNTs to successfully bridge the electrodes. By reducing the electrode spacing the yield of working devices was increased.

By reducing the physical distance between the CNT/electrode interface in method I and having good contact with the CNT and the the electrode, field dependent devices were made, over the gate field range $\mathrm{Vg}-5 \mathrm{~V}$ to $+5 \mathrm{~V}$. The device reproducibility and behaviour is seen to depend on the initial CNT suspensions. For any given suspension, CNT films were observed to be similar and Ids did not vary at higher Vg. However, the ratio of metallic and semiconducting CNTs could not be homogenised, and as such dramatically altered the overall device characteristics.

By stamping over predefined electrodes, method II, where an increased physical dis- 
tance between the CNT/electrode interface existed, devices were observed to act as metallic conductors with little or no gate dependence, over the gate field range $\mathrm{Vg}-5 \mathrm{~V}$ to $+5 \mathrm{~V}$. By increasing the gate voltage to $-20 \mathrm{~V}$, p-type field dependence was observed.

The interesting outcome of these experiments has been to show that CNTs can be self-assembled using $\pi$ bond interactions, to produce uniform CNT films for high device yields. Such $\pi$ bond interactions make the molecular stamping and self-assembly method highly versatile and suitable for both pristine and functionalised CNTs. By altering the order of the fabrication process devices can be either field-dependent or not. No selection of CNTs between metallic and semiconducting has been required. The magnitude of the current in each device depends largely on the original concentration of the CNT solution and at present is still irregular between similarly dispersed CNTs solutions.

It is therefore envisioned that by further control of the CNT dispersion and a shrinking of the oxide layer tailored CNT devices can be fabricated. 


\section{Chapter 9 Conclusion}

The work carried out in contribution to this thesis has exploited the use of chemistry based techniques to control carbon nanotubes (CNTs) for electronic device applications. Chemical functionalisation techniques have been applied to convert the normally unreactive CNTs to reactive molecules and to create reactive substrates for CNT adhesion.

Plasma fluorination was developed to increase the reactivity of the CNTs and to allow further functionalisation by amine groups. The benefits of such amine functionalisation is in the ability of the amine group to donate electrons to the CNTs structure, making the inherently p-type CNTs, towards n-type conductors. Plasma thiolation techniques were also developed to control the positioning of CNTs on substrate surfaces. The thiolated CNTs would self-assemble due to the spontaneous formation of a gold-thiol bond. Finally pristine CNTs have been self-assembled over a substrate using $\pi$ bond interactions between carbon carbon double bonds of the CNTs and a stamped pyridine molecule layer, to produce a high yield CNT device fabrication process.

The main conclusions from each section will now be presented and the developments for future work discussed.

\subsection{Plasma Fluorination of Carbon Nanotubes}

The first step to CNT electronic devices has been to control some of the properties of the CNTs by chemical modifications. The inherent CNT structure is chemically stable and behaves as a p-type field effect device. A plasma fluorination process was developed to produce CNTs with functional groups capable of undergoing further reactions. Due to the high reactivity of fluorine many further reactions could be carried out on fluorinated CNTs, (F-CNTs).

It has been shown that fluorination of carbon nanotubes by plasma exposure has been a reliable means for producing functionalised carbon nanotubes and remained stable 
over time, both with a $\mathrm{CF}_{4}$ plasma and a $\mathrm{SF}_{6}$ plasma. The fluorination was characterised using several spectroscopic methods. XPS and Raman spectroscopy were used to determine the bonding nature of the fluorine on the CNT surface and the CNT structural integrity respectively. In particular the plasma environment has allowed for the study of the mechanism of CNT functionalisation, by use of plasma diagnostic tools the Langmuir probe and optical emission spectroscopy. It has been shown that the fluorination of the CNTs is sensitive to the reactor conditions.

Such knowledge of the functionalisation mechanism has been useful for the further functionalisation of CNTs. The $\mathrm{CF}_{4}$ plasma exposed CNTs were observed to have an increased stoichiometry of $\mathrm{F} / \mathrm{C}$ in comparison to the $\mathrm{SF}_{6}$ plasma exposed $\mathrm{CNTs}$, however there was also an increased $\mathrm{O} / \mathrm{C}$, both due to the increase in ion current density in the $\mathrm{CF}_{4}$ plasma environment. The $\mathrm{SF}_{6}$ plasma exposed CNTs have a more covalent $\mathrm{C}-\mathrm{F}$ bond over all exposures tested, due to the increase in fluorine radicals available in the plasma in comparison to the $\mathrm{CF}_{4}$ plasma exposed CNTs.

CNTs can withstand the somewhat aggressive nature of a plasma environment and maintain their structural integrity as verified by Raman spectroscopy. The plasma fluorination process has shown advantages over previous CNT fluorination due to the dramatically reduced exposure times and without the need to functionalise at high temperatures $[8-10,118,158]$. The plasma diagnostic tools have also allowed for the mechanism of fluorination in these processes to be deduced, providing useful information for the choice of further functionalisation procedures.

\subsection{Amine Functionalisation of Fluorinated Carbon Nanotubes}

CNTs functionalised in a $\mathrm{CF}_{4}$ plasma, F-CNTs, have been readily applied for further functionalisation experiments by substitution reactions. Here, 1,2-diaminoethane was used to substitute the fluorine atoms bonded to the CNT surface to create N-CNTs. The 1,2-diaminoethane acts as an electron donator and as such can change the CNT device behaviour from p-type to n-type. 1,2-diaminoethane was chosen due to the double amine group as it was believed that the extra amine would further enhance the n-type behaviour of the N-CNTs.

The substitution of fluorine for 1,2-diaminoethane was shown to depend on the initial 
concentration of the fluorination of the F-CNTs. As such it could therefore be concluded that the substitution did take place at fluorine sites rather than plasma induced and oxygen defects on the CNT structure. The reaction was predicted to take place as a bi-functionalisation process, with increasing initial fluorine content increasing the likelihood of bi-functionalisation.

Probing the electronic behaviour of both the F-CNTs and the N-CNTs in back gate geometry has shown that the amine molecules do donate electrons to the CNT structure. However, the low stoichiometry of the doping has provided only weak n-type conducting effects over the range of gate voltages swept here and left the devices in a regime where the CNT/electrode contacts dominate the conduction [95].

Previous work by other authors had chemically doped CNTs using amine molecules to control the CNT chemistry $[14-16,92,94,95]$. Here the control of the initial fluorination stage is an advantage to produce CNT devices with known functionalisation parameters, similar to other work $[15,94]$ although the plasma fluorination is a simpler and faster initial functionalisation process. By refining the fluorination process the stoichiometry and therefore the electronic behaviour of the CNTs should be controlled. However, problems remain in the initial CNT suspensions containing both metallic and semiconducting CNTs and the tendency for the CNTs to bundle together. Such suspension problems do however affect all work on CNT electronics and is not an exclusive problem to this work.

Further fluorination and amine functionalisation experiments are underway at the time of writing. The emphasis is on the deduction of the ionic and covalent components of the initial fluorination and the effect on the subsequent amine functionalisation and I-V behaviour.

\subsection{Direct Thiolation of Carbon Nanotubes}

Due to the known reactivity of the plasma environment and the ability to fluorinate CNTs without loss of CNT structure, a second plasma functionalisation process was developed to directly thiolate the CNT sidewalls. Direct thiolation was developed to allow positional control of the CNTs onto a substrate featured with Au electrodes. Due to the spontaneous formation of the Au thiol bond, CNTs were observed to self-assemble 
onto the substrates.

To achieve the direct thiolation of CNTs, the heating of the sulphur and the addition of a $\mathrm{H}_{2}$ feed stock gas in a bulk argon plasma were both essential. The plasma chemistry has also been advantageous for the thiolation without the addition of excess oxygen contaminants which are known to alter the electrical behaviour of the CNTs.

SEM has confirmed that after direct plasma thiolation CNTs would self-assemble to bridge narrow gaps between Au electrodes. Previous work by other groups had thiolated CNTs with the use of long and short chain carbon groups by conventional wet chemical functionalisation techniques [33-35]. After such techniques the CNTs were often shortened and self assembled perpendicular to any Au surfaces. Here the CNTs have experienced less structural damage as there was no acid oxidation process employed for wet chemical functionalisation and therefore no cutting of the CNTs. The reaction dynamics of the plasma thiolated CNT self-assembly process in relation to the chain length of the carbon nanotubes appear to have been advantageous in these experiments, allowing the CNTs to bridge Au electrodes over an oxide region.

The electronic characterisation of the self-assembled thiolated CNTs has shown that the CNT devices vary considerably depending upon the bundle formed. Devices have been observed to behave as metallic conductors and p-type field dependent devices. The suspension of CNTs is again thought to be limiting the device uniformity and the yield of working devices.

It is proposed that further improvements to the CNT suspensions and the patterning of $\mathrm{Au}$ microdots between electrode structures could be used to optimise the thiolated CNT self-assembly process and increase the device yield.

\subsection{Molecular Stamping, for Carbon Nanotubes Device Fabri- cation}

Methods to self-assemble pristine CNTs onto substrate surfaces have also been developed by the use of molecular stamping techniques. The molecular stamping techniques developed here, resulted in high yield of CNT devices of 90\%. PDMS stamps were fabricated and used to stamp 2-thiolpyridine. The Au-thiol (and also $\mathrm{SiO}_{2}$-thiol) at one end 
of the stamped molecule provided even coverage across the substrate surface, leaving the pyridine end of the molecule available for CNT attachment via $\pi$ bond interactions. Several parameters must be optimised for the stamping process to provide successful self-assembly conditions for CNT device applications. The important parameters include the concentration of the 2-thiolpyrinde solution, the suspension of the CNTs and finally the electrode spacing.

Other self-assembly techniques for selective positioning of CNTs have required aminesilane layers $[170,171]$ and polar molecules [38]. The fabrication process outlined with the stamping of 2-thiolpyridine, is an advantage over these other methods $[38,170,171]$ due to the simplicity of the steps and the high throughput such a technique can allow. Here, the CNT hexagonal carbon $\pi$ bonds are the attractive force for the CNT self-assembly, making the use of 2-thiolpyrinde suitable for both p-type and n-type functionalised CNTs, at low stoichiometries.

The alteration of the fabrication process between electrodes on top of CNTs and electrodes below the CNTs has provided useful insight into the gate screening effects of the stamped 2-thiolpyridine layer. It was shown that by altering the order of the fabrication process devices can be either field-dependent or not over the gate voltage range -5 to $+5 \mathrm{~V}$. In method I, where electrodes are patterned directly on top of CNTs allowing for good contact between the CNT and the electrode, field dependent devices were made over the gate field range $\mathrm{Vg}-5 \mathrm{~V}$ to $+5 \mathrm{~V}$. By stamping 2-thiolpyridine and self-assembling CNTs over predefined electrodes in method II, an increased physical distance between the CNT/electrode interface existed. Such devices were observed to act as metallic conductors with little or no gate dependence, over the gate field range Vg -5 $\mathrm{V}$ to $+5 \mathrm{~V}$. By increasing the gate voltage to $-20 \mathrm{~V}$, p-type field dependence was observed for method II type devices. No selection of tubes between metallic and semiconducting has been required.

The magnitude of the current in each device depends largely on the original concentration of the CNT solution and at present is still irregular between similarly dispersed CNTs solutions, although similar on any given chip. So far the method has been applied for pristine CNTs only, however, the fabrication process and self-assembly mechanism is transferable to functionalised CNTs. 
It is therefore envisioned that by further control of the CNT suspensions and a shrinking of the oxide layer tailored CNT devices can be fabricated. By applying burn-off to the fabricated device structures, purely semiconducting devices may be realised. The stamping techniques are also useful for a scaled down version of the devices, provided that small contact electrodes can be made lithographically.

\subsection{Further Work}

Although this work has provided no definitive cure for the problems in CNT device technology, all method used have shown promise and progress towards the next generation of electronic devices. It is important to realise that the microelectronics industry requires, more than ever, the use of complex chemical methods and the use of bottom up based solutions previously unseen to meet the miniaturisation demands the field requires. The development of such techniques are still at an early stage, however, it is an area in which microelectronic engineers are becoming more proficient.

The characterisation and fabrication stages developed here have been a valuable step towards CNT devices, notably by the publication of several papers in the field $[17,18$, 175], and others accepted but yet to be published, appendix A. A common problem for all fabrication processes discussed here was the ability to produce CNT suspensions of high and repeatable quality. If a batch of CNTs was grown to specific chirality, the methods used here could be easily employed to create reproducible results. At present there are too many statistical differences within the CNT suspension to accurately predict the device behaviour.

Suspensions improvements would help both with the functionalisation experiments and the positional control aspects of the CNT devices. Yet, even with the current suspension limitations further improvements can be made to the CNT devices. Further characterisation of the ionic and covalent components of the C-F bonds in the F-CNTs, for further chemical doping should provide greater control of any n-type CNT device behaviour. Also, application of the molecular stamping self-assembly technique should be applicable to the n-type CNTs to provide more uniform CNT distribution on the substrate surfaces for comparative electronic analysis.

Improved micropatterning techniques for small gold nanodots between electrode struc- 
tures in the case of the thiolated CNTs may improve the device yield. Also the microfabrication of smaller electrodes and miniaturised PDMS stamps could be readily applied to scale down the molecular stamping devices. An accurate pressure control during the stamping stage and a strengthening of the PDMS stamp would both be useful to avoid CNTs self-assembly out with the predefined electrode regions.

Precise device characteristics can also be controlled by applying burn-off techniques and controlling the gate oxide thickness. Here the CNT FETs did not reach saturation largely due to the thick gate oxide. Thinning the oxide layer would allow further device characterisation to be be made. Also, by locally applying a gate directly above the CNTs individual devices may be probed and CMOS device structure could be fabricated. Such experiments could elucidate further CNT characteristics behaviour due to the interaction between the CNTs and the functional chemicals used here.

In conclusion it has been shown that pre-grown CNTs can be controlled by functionalisation processes, both direct, in the sense of the plasma functionalisation methods, and indirect during the molecular stamping. All processes were carried out in parallel to common microfabrication techniques, without loss of CNT structure. The techniques developed here show ample promise for refinement for future CNT device fabrication. 


\section{Appendix A Papers}

During the course of the research undertaken towards this thesis some of the work has been published in relevant journals and presented at international conferences. The peer reviewed journal publications are reprinted in this appendix and referenced in chronological order.

N. O. V. Plank, Liudi Jiang and R. Cheung, "Fluorination of carbon nanotubes in $\mathrm{CF}_{4}$ plasma', Applied Physics Letters, 83, 2462 (2003)

N. O. V. Plank and R. Cheung, "Functionalisation of carbon nanotubes for molecular electronics", Microelectronic Engineering, 73-74, 578 (2004)

N. O. V. Plank, R. J. Andrews and R. Cheung, "Thiolation of carbon nanotubes and their self-assembly", Applied Physics Letters, 85, 3229 (2004)

N. O. V. Plank, Masahiko Ishida and R. Cheung, "Positioning of carbon nanotubes using soft-lithography for electronics applications", accepted for publication by Journal of Vacuum Science and Technology B, September (2005)

Natalie O. V. Plank, Gavin A. Forrest, Rebecca Cheung and Andrew J. Alexander "n-type carbon nanotube devices prepared by $\mathrm{CF}_{4}$ plasma-fluorination and amino-functionalization", accepted for publication by Journal of Physical Chemistry B, October (2005) 


\title{
Fluorination of carbon nanotubes in $\mathrm{CF}_{4}$ plasma
}

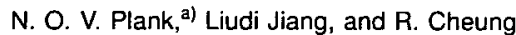 \\ School of Engineering and Electronics, University of Edinburgh, The Sconish Microelectronics Centre, \\ Mayfield Road, King's Buildings, EH9 $3 J$ L Scotland, United Kingdom \\ (Received 9 April 2003; accepted 21 July 2003) \\ The effect of $\mathrm{CF}_{4}$ gaseous plasma exposure to single-wall carbon nanotubes (CNTs) has been \\ studied. Raman spectroscopy results show that CNTs have gained more disordered $s p^{3}$ bonds \\ associated with functionalization, as both the flow rates of gas in the plasma and exposure time in \\ the plasma are increased. Scanning electron microscopy images indicate the CNTs have been \\ preserved after $\mathrm{CF}_{4}$ plasma exposure. $\mathrm{X}$-ray photoelectron spectroscopy provides evidence of \\ carbon to fluorine bonds $(\mathrm{C}-\mathrm{F})$ on the $\mathrm{CNTs}$ samples after $\mathrm{CF}_{4}$ plasma exposure. Semi-ionic and \\ covalent $\mathrm{C}-\mathrm{F}$ bonds are prevalent on the $\mathrm{CNTs}$ after $\mathrm{CF}_{4}$ exposure with the intensity ratio of the \\ semi-ionic to covalent $\mathrm{C}-\mathrm{F}$ bond decreasing as the flow rate of $\mathrm{CF}_{4}$ and exposure time in the $\mathrm{CF}_{4}$ \\ plasma is increased. (c) 2003 American Institute of Physics. [DOI: 10.1063/1.1611621]
}

Since their discovery in $1991^{1}$ great interest has been shown in carbon nanotubes (CNTs) both in the theoretical understanding and in practical applications, ${ }^{2}$ due to the physical and electrical properties of the material, ${ }^{3}$ The conduction properties of carbon nanotubes have been studied previously, as single conducting wires ${ }^{4}$ and as more complex device structures such as field effect transistors. ${ }^{5}$ An important step that will lead to more practical use of the naturally stable nanotubes is the process of functionalization. Functionalized nanotubes are chemically more reactive and can be employed for further processing such as thiolization ${ }^{6}$ or for integration in nanocomposites. ${ }^{7}$ Moreover, tuning of the electrical characteristics of nanotubes from metallic to semiconducting is possible depending on the degree and nature of functionalization. ${ }^{8.9}$ However, functionalization of the CNTs is a complex step and has been carried out using a number of different techniques. ${ }^{10-16}$ Recently, a glow discharge has been used to attach hydrogen to the sidewalls of carbon nanotubes. ${ }^{17.18}$ Plasma processing is known to modify surfaces at an atomic scale. ${ }^{19}$ For example, various types of $\mathrm{C}-\mathrm{F}$ bonding have been found on silicon carbide surfaces after exposure to fluorinated plasma. ${ }^{20}$ In this work, we demonstrate the fluorination of single wall CNTs using $\mathrm{CF}_{4}$ plasma. The advantages of CNT functionalization using plasma exposure are the short duration of time needed and the room temperature processing ability in comparison to previous methods, which often require long process times and high temperature treatment.

Single wall CNTs, from Carbon Nanotechnologies Inc were prepared by dispersion in isopropanol (IPA), at a concentration of $5 \mathrm{mg}$ of CNTs to $15 \mathrm{ml}$ of IPA, using a mag. netic stirrer for $2 \mathrm{~h}$ at room temperature resulting in the formation of a thick black paste. The dispersion was then filtered through a $2 \mu \mathrm{m}$ polytetrafluoroethylene (PTFE) filter membrane from Millipore under vacuum and left to dry for $24 \mathrm{~h}$ at room temperature, resulting in the formation of "bucky papers" on top of the PTFE membranes. Subsequently, the samples were exposed to $\mathrm{CF}_{4}$ plasmas with 20

"Llectronic mail: natalie.plank@ee.ed.ac.uk and $40 \mathrm{sccm}$ flow rates for $30 \mathrm{~s}, 1$, and $2 \mathrm{~min}$ in a Vacutech reactive ion etcher, with a chamber pressure of $0.15 \mathrm{~Pa}$ at 20 sccm and $0.196 \mathrm{~Pa}$ at $40 \mathrm{sccm}$. All samples were exposed to the plasmas with a dc bias of $-300 \mathrm{~V}$. The nonplasmaexposed sample is referred to as the control sample throughout the text. To characterize the effect of the plasma on the nanotubes, Raman spectroscopy, scanning electron microscopy (SEM), and x-ray photoelectron spectroscopy (XPS) have been used.

The Raman spectra of the control and $\mathrm{CF}_{4}$ plasma exposed CNTs are shown in Fig. 1. The shape of the spectra remains unchanged after plasma treatment with the intensity of the lines being altered. Both the $G$ line (at around $1580 \mathrm{~cm}^{-1}$ ) corresponding to $s p^{2}$ bonded carbon, characteristic of the $\mathrm{C}-\mathrm{C}$ bond in carbon nanotubes and the $D$ line (at around $1375 \mathrm{~cm}^{-1}$ ) corresponding to $s p^{3}$ bonding, relating to scattering defects in the CNT samples are evident in the spectra. Our Raman spectra are characteristic of CNTs when compared with the Raman spectra of amorphous carbon, glassy carbon, and carbon black ${ }^{21}$ as well as other Raman studies of CNTs, 14,21

The relative intensity ratios of the $D-G$ bands shown in

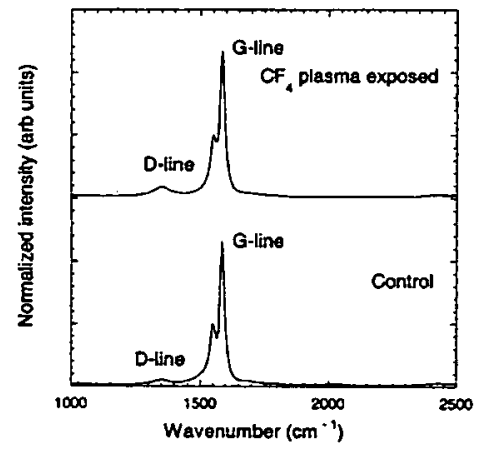

FIG. I. Raman specura of control and $\mathrm{CF}_{4}$ plasma exposed single wall CNTs.

Figure A.1: Reprint of APL vol 83, p2462 


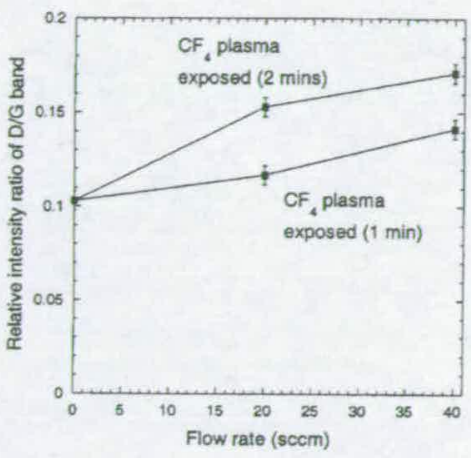

FIG. 2. Relative intensity ratios of the $D-G$ bands of $\mathrm{CF}_{4}$ plasma exposed CNTs as a function of flow rate of $\mathrm{CF}_{4}$ in the plasms.

Fig. 2 give an indication of the degree of disorder in the carbon nanotubes. The weak $D$ band at approximately $1300 \mathrm{~cm}^{-1}$ has previously been correlated to the extent of sidewall functionalization, ${ }^{14}$ it is highly likely that the same effect occurs on our samples. The general trend is that as the flow rate of the gas increases, a larger degree of disorder, functionalization is observed. Moreover, exposing the carbon nanotubes to $\mathrm{CF}_{4}$ plasma for $2 \mathrm{~min}$ results in a larger degree of disorder compared to exposure for $1 \mathrm{~min}$. It should be noted here that the $G$ band has stayed strong throughout indicating that a large proportion of ordered nanotubes remain. It is worth noting that the extreme treatments for fluorine or oxygen attachment in other works have often caused the significant broadening of the $D$ and $G$ peaks. ${ }^{13-16}$ Additionally, energies up to $100 \mathrm{keV}$ are reported to be necessary to damage nanotubes, ${ }^{22}$ it is believed that at the typical energies involved in the plasma process $\sim 300 \mathrm{eV}$, the nanotubes would not have been damaged although they may be slightly modified, as confirmed by the Raman study. Figure 3 shows the SEM images of the control and $\mathrm{CF}_{4}$ plasma exposed bucky papers. Both surfaces look very similar suggesting that subtle changes have been introduced to the nanotubes after $\mathrm{CF}_{4}$ plasma exposure.

XPS has been used as a means of detecting chemical species attached to the CNTs. Figure 4 shows the normalized $\mathrm{F}$ Is spectra of the control and $\mathrm{CF}_{4}$ plasma exposed tubes. The presence of fluorine on the bucky papers is detected only on the $\mathrm{CF}_{4}$ plasma exposed tubes. The nature of the carbon to fluorine bonds $(\mathrm{C}-\mathrm{F})$ has been analyzed by deconvoluting the $\mathrm{F} 1 s$ peak of the $\mathrm{CF}_{4}$ plasma exposed CNTs with Gaussian peaks after subtraction of linear mode backgrounds. Both semi-ionic $(685.5 \mathrm{eV})$ and covalent $(687.5 \mathrm{eV}) \mathrm{C}-\mathrm{F}$ bonds ${ }^{20,23,24}$ have been clearly observed. There is also a third peak observed in the $\mathrm{F} 1 s$ spectra of the $\mathrm{CF}_{4}$ plasma exposed nanotubes at $689.2 \mathrm{eV}$, possibly due to the presence of $p-\left(\mathrm{CF}_{2}=\mathrm{CF}_{2}\right)$ bonds ${ }^{25}$ The occurrence of the fluorinated carbon bonds is believed to be due to the plasma chemistry.

The intensity ratios of semi-ionic and covalent $\mathrm{C}-\mathrm{F}$ bonds $\left(I_{\text {semi-ionic }} / I_{\text {covalent }}\right)$ from the $\mathrm{CF}_{4}$ plasma exposed CNTs are shown in Table I. It is noticed that the presence of covalently bonded $\mathrm{C}-\mathrm{F}$ increases as the $\mathrm{CF}_{4}$ gas flow rate is increased during $\mathrm{CF}_{4}$ plasma exposure and/or with increase

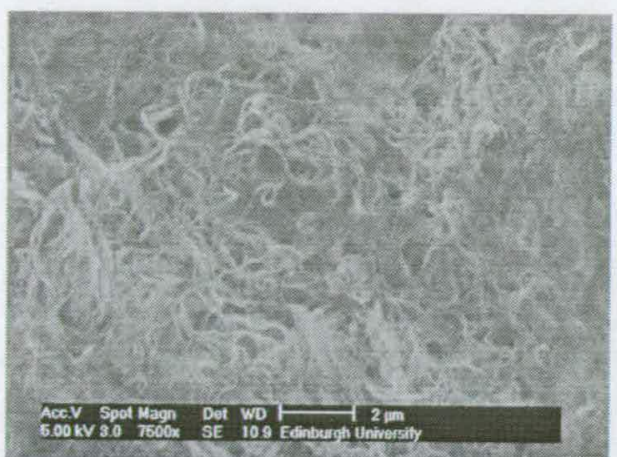

(a)

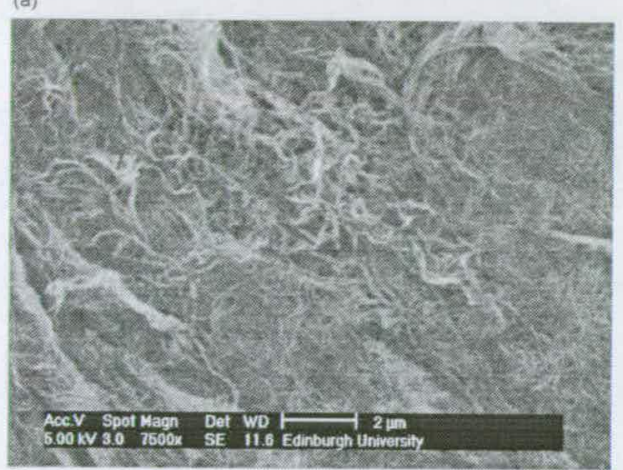

(b)

FIG. 3. Scanning electron micrographs for (a) control CNTs and (b) CNTs after $\mathrm{CF}_{4}$ plasma exposure with $40 \mathrm{sccm}$ flow rate for $1 \mathrm{~min}$.

with exposure time. The smallest ratio is observed for the highest $\mathrm{CF}_{4}$ flow rate and maximum exposure time under study. This observation is commensurate with the fact that covalently bonded $\mathrm{C}-\mathrm{F}$ occurs when the localization of pi electrons of the $\mathrm{C}$ atoms increases strongly as a result of an increase in the $\mathrm{F}$ density, causing the $\mathrm{F}$ atoms to get closer to the $\mathrm{C}$ atoms. ${ }^{15,23}$ It should be noted that the $\mathrm{F} 1 s$ and $\mathrm{C} 1 s$

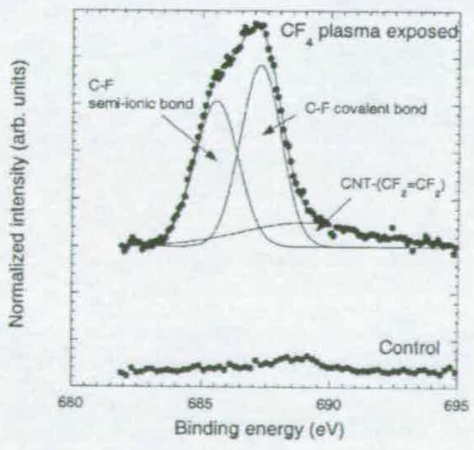

FIG. 4. X-ray photoelectron spectra of F is peak for control CNTs and CNTs after $\mathrm{CF}_{4}$ plasma exposure with 40 scem flow rate for 1 min.

Figure A.2: Reprint of APL vol 83, p2463 
TABLE I. / Isemi-inax $/ /$ conzlem intensity ratio for C-F bonds, derived from the $F$ is spectra as a function of $C_{4}$ flow rate and plasma exposure time.

\begin{tabular}{lcc}
\hline \hline Flow rate & $20 \mathrm{secm}$ & $40 \mathrm{secm}$ \\
\hline Time (min) & & \\
1 & 0.888 & 0.848 \\
2 & 0.810 & 0.639 \\
\hline
\end{tabular}

peaks observed during this study are similar to those found during the fluorination of CNTs by An et al. ${ }^{15}$ at low temperatures $\left(150^{\circ} \mathrm{C}\right)$ where the CNTs had a F/C ratio $<0.5$. The highest $\mathrm{F} / \mathrm{C}$ ratio found in our $\mathrm{CF}_{4}$ plasma exposed CNTs has been 0.22 , which is less than the theoretical and experimental saturation stoichiometries of $\mathrm{C}_{2} \mathrm{~F}^{13}$ or $\mathrm{CF}_{0.5}{ }^{15}$ The low stoichiometry, coupled with the subtle Raman changes observed indicate the possibility that the $F$ atoms are chemisorbed at the outer surface of the CNT wall..$^{15.23}$

The authors would like to thank Ronald Brown with assistance in the XPS measurements and Francesca Fabbiani for assistance in the Raman spectroscopy measurements.

'S. lijima, Nature (London) 354, 56 (1991).

${ }^{2} \mathrm{H}$. Dai, Surf. Sci, 500, $218(2002)$.

${ }^{3}$ S. J. Tans, A. R. M. Verschueren, and C. Dekker, Nature (London) 393, 49 (1998).

${ }^{4}$ C. Zhuu, J. Kong, and H. Dai, Appl. Phys. Lell. 76, 1597 (2000)

'R. Saito, G. Dresselhaus, and M. S. Dresselhaus, Physical Properties of Carbon Nanotubes (Imperial College Press, London, 2001)

${ }^{\circ} Z$. Liu, Z. Stren, T. Zhu, S. Hou, and L. Ying, Langmuir 16, 3569 (2000).
'Y.-P. Sun. K. Fu, Y. Lin, and W. Huang, Acc. Chem. Res. 35, 1096 (2002). ${ }^{8}$ G. Seirfet, T. Kohler, and T. Frauenheim, Appl. Phys. Lett. 71, 1313 (2000).

${ }^{9}$ K. N. Kudin, H. F. Bettinger, and G. E. Scuseria, Phys. Rev. B 63, 045413 (2001).

${ }^{10}$ A. Hirsch, Angew. Chem., Int. Ed. 41, 1853 (2002).

"S. B. Sinnon, J. Nanosci. Nanotechnol. 2, 113 (2002).

${ }^{12}$ V. N. Khabashsku, W. E. Billups, and J. L. Margrave, Acc. Chem. Res. 35, 1087 (2002).

${ }^{13}$ E. T. Mickelson, C. B. Huffman, A. G. Rinzler, R. E. Smalley, R. H. Hague, and J. L. Margrave, Chem. Phys. Lett. 296. 188 (1998).

is S. Banerjee and S. S. Wong, J. Phys. Chem. B 106, 12144 (2002)

${ }^{15}$ K. H. An, J. G. Heo, K. G. Jeon, D. J. Bac, C. Jo, C. W. Yang, C.-Y, Park, Y. H. Lee, Y. S. Lee, and Y. S. Chung, Appl. Phys. Lett. 80, 4235 (2002).

${ }^{16}$ P. R. Marcoux, J. Schriher, P. Batail, S. I efrant, J. Rennuard, G. Jacoh, D. Albertini, and J.-Y. Mevellec, Phys. Chem. Chem. Phys. 4, 2278 (2002). ${ }^{17}$ B. N. Khare, M. Meyyappan. A. M. Cassell. C. V. Nguyen, and J. Han, B. N. Khare, M. Meyy

Nano Lett. 2, 73 (2002).
${ }_{18}$ B. N. Khare, M. Meyyappan, J. Kralj, P. Wilhite, M. Sisay, H. Imanakia, J.

${ }^{18}$ B. N. Khare, M. Meyyappan. J. Kralj, P. Wilhite, M. Sisay, H. Imanaki,
Koehne, and C. W. Baushchitcher, Appl. Phys. Let. 81, 5237 (2002).

19. A. Mucha and D. W. Hess, in Introduction to Microlithography (Wash-

ington American Chemical Sociely. Washington, 1983). pp. 215-285.

${ }^{20}$ L. Jiang. R. Cheung, R. Brown, and A. Mount, J. Appl. Phys. 93, 1376 (2003).

${ }^{21}$ H.-D. Klotz, R. Mach, F. Oleszak, H.-E. Maneck, H. Goering, and K.-W. Brıezinka, Appl. Surf. Sci. 179, I (2001).

"v. Krasheninnitov. K. Nardlund. J. Keinonen. and F. Banhar, Phys. Rev. B 66, 245403 (2002)

B 66,245403 (2002).

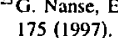

2ะ Y. Hattori, Y. Walanabe, S. Kawaski, F. Okino, B. K. Pradhan, T. Kyolani.

${ }^{2}$ Y. Hattori, Y. Walanabe, S. Kawaski, F. Okino, B. K. Prot
A. Tomila, and H. Touhara, Carton 37, 1033 (1999).

2s J. F. Moulder, W. F. Stickle, P. E. Subol, and K. D. Bomben, Handbook of $X$-Ray Photoelectron Spectroscopy (Perkin-Elmir, Eden Prairie, MN, 1993).

Figure A.3: Reprint of APL vol 83, p2464 


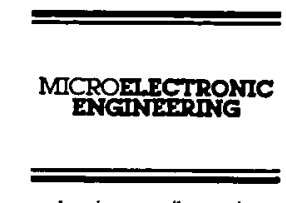

\title{
Functionalisation of carbon nanotubes for molecular electronics
}

\author{
N.O.V. Plank *, R. Cheung
}

School of Engineering and Electronics, Scontish Microelectronics Centre. University of Edinburgh. The King's Buildings, West Mains Road, Edinburgh EH9 3JL. Scotland, UK

Available online 14 March 2004

\section{Abstract}

Single-walled carbon nanotubes (SWNTs) have been functionalised using a conventional Reactive Ion Etching (RIE) system with $\mathrm{CF}_{4}$ and $\mathrm{SF}_{6}$ plasmas. The fluorinated $\mathrm{SWNTs}$ have been investigated using $\mathrm{X}$-ray photoelectron spectroscopy. Microscopic plasma parameters including the fluorine pressure and the ion current density within the plasma have been studied using optical emission spectroscopy and a Langmuir probe. It has been observed that a bias is required for successful attachment of fluorine to the SWNTs in the plasma environment. The mechanism of fluorine attachment to SWNTs is evaluated by correlating the microscopic plasma parameters with the Fls binding energy, the fluorine/carbon ratio and also the oxygen/carbon ratio on the SWNTs. The attached fluorine has been seen to remain stable over time, making the plasma process a reliable means of fluorine functionalisation of carbon nanotubes. (c) 2004 Elsevier B.V. All rights reserved.

Keywords: SWNTs; XPS; OES; Langmuir probe and fluorination

\section{Introduction}

Single-walled carbon nanotubes (SWNTs) show outstanding potential for uses in molecular electronics. Not only are the nanotubes inherently small, they also offer exceptional versatility due to their unique electronic properties, as SWNTs can be either metallic or semiconducting depending upon how the tubes have rolled up along the graphite plane [1]. As a possible first step in the

\footnotetext{
Corresponding author.

E-mail address: Natalie.Plank@ee.ed.ac.uk (N.O.V. Plank).
}

utilisation of SWNTs in molecular electronics, it is necessary to functionalise the relatively unreactive tubes. Previous studies have involved long acid oxidation stages [2] to functionalise the tubes with carboxylic acid groups and long gas fluorination processes [3,4]. Recently Khare et al. $[5,6]$ have used a glow discharge to functionalise carbon nanotubes with hydrogen and previous work by the authors have employed a conventional Reactive Ion Etcher (RIE) reactor to attach fluorine to SWNTs using a $\mathrm{CF}_{4}$ plasma [7] in a functionalisation process of the order of minutes. Here a $\mathrm{CF}_{4}$ plasma and a $\mathrm{SF}_{6}$ plasma have been compared at the high bias regime of the reactor, with emphasis

0167-9317/\$ - see front matter (c) 2004 Elsevier B.V. All rights reserved. doi:10.1016/j.mee.2004.02.088

Figure A.4: Reprint of Microelectronic engineering vol 73-74, p578 
on the chemical attachments to the SWNTs and the plasma chemistry.

\section{Experimental method}

Single walled carbon nanotubes ${ }^{1}$ were used throughout this work. Twenty milligrams of the nanotubes were dispersed in $20 \mathrm{ml}$ of isopropyl alcohol using a magnetic stirrer bar for $2 \mathrm{~h}$. The solution was then filtered through a $1 \mu \mathrm{m}$ PTFE membrane under vacuum. The membranes were then left to dry, resulting in the adhesion of a bucky paper to the top of the membrane.

The bucky papers were then exposed to a plasma environment within the Vacutech RIE system using either $\mathrm{SF}_{6}$ at $20 \mathrm{sscm}$ flow rate or $\mathrm{CF}_{4}$ at $40 \mathrm{sccm}$ flow rate as the plasma gas. The samples were exposed to the plasma for $1 \mathrm{~min}$ at different bias voltages to determine the effect of bias on the subsequent bonding of fluorine to the SWNTs. The chemical attachments were investigated by X-ray Photoelectron spectroscopy ${ }^{2}$ (XPS) using a monochromatic $\mathrm{Al}(\mathrm{K} \alpha)=1486.6 \mathrm{eV}$ source. The Fls peaks have also been studied with emphasis on the binding energy, using XPS Peak software whereby subtraction of a linear background is performed and Gaussian curves are fitted to the measured data. Attention has been paid to the relative concentrations of fluorine to carbon and oxygen to carbon and after subtraction of a linear background and with use of AlWagner coefficients in the Avantage data system, both 2 days after the initial functionalisation and also after 4 weeks. The AlWagner coefficients are used to limit any possible atomic percentage errors which may occur due to the sample preparation and positioning in the XPS equipment.

The reactor conditions are characterised with use of optical emission spectroscopy (OES) and Langmuir probe measurements. The OES data confirms the relative concentrations of radical fluorine within the plasma, whilst the Langmuir probe is used to determine the ion current density,

\footnotetext{
1 Purchased from CNI

2 Thermo VG Scientific Sigma Probe.
}

hence relating to the ion bombardment factors taking place on the bucky paper surface.

Transmission electron microscope (TEM) im. ages of the SWNTs are also taken to test the structural integrity of the SWNTs after plasma exposure. For the TEM images, $2 \mathrm{mg}$ of SWNT bucky paper were suspended in $2 \mathrm{ml}$ of IPA and a drop of the solution was then suspended onto 300 $\mu \mathrm{m}$ carbon grids.

\section{Results and discussion}

The Fls region of SWNTs exposed to the $\mathrm{CF}_{4}$ plasma the $\mathrm{SF}_{6}$ plasma and the unexposed control sample are shown in Fig. 1. The Fls peak of the SWNTs functionalised in the $\mathrm{CF}_{4}$ plasma and the $\mathrm{SF}_{6}$ plasma, have been deconvoluted using XPS Peak software. The tubes functionalised with $\mathrm{CF}_{4}$ plasma require two large Gaussian peaks to obtain a good fit with the measured data, one at $686 \mathrm{eV}$ in the semi-ionic region and another at $687.5 \mathrm{eV}[8,9]$ in the covalent $\mathrm{C}-\mathrm{F}$ bonding region, over all exposure conditions. The SWNTs functionalised in $\mathrm{SF}_{6}$ plasma however need only one large peak at $687 \mathrm{eV}$, towards the covalent region of the Fls spectra to fit the measured data, again over all exposure conditions. The broad low intensity peak at $689.5 \mathrm{eV}$ is present after both functionalisation

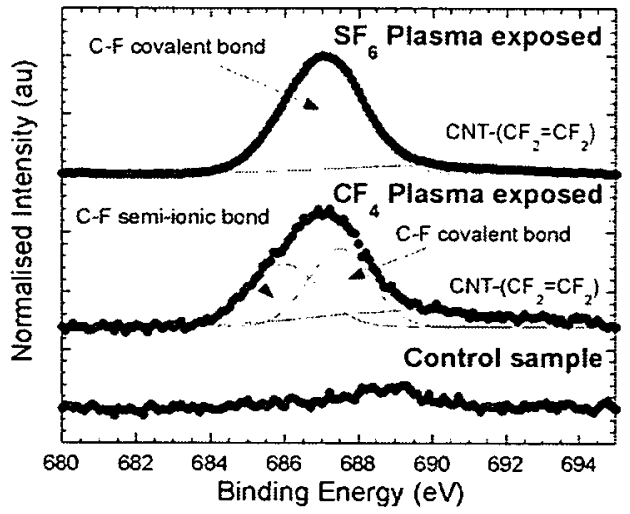

Fig. 1. The deconvoluted $\mathrm{F} l s$ peak for the $\mathrm{SF}_{6}$ and $\mathrm{CF}_{4}$ plasma exposed SWNTs at $-200 \mathrm{~V}$ for $1 \mathrm{~min}$, in comparison to the unexposed control sample.

Figure A.5: Reprint of Microelectronic engineering vol 73-74, p579 
procedures and is thought to be due to weak sputtering effects within the plasma environment.

The possible mechanism for this behaviour has been studied using OES and Langmuir probe measurements. The results from the OES have been used to calculate the fluorine pressure within the reactor using an actinometry technique described previously [10]. The fluorine pressure as a function of bias voltage is shown in Fig. 2(a). The result at $-0 \mathrm{~V}$ bias refers to the flow of $\mathrm{SF}_{6}$ and $\mathrm{CF}_{4}$ process gases, respectively, as the plasma environment could not be maintained without application of a bias voltage and therefore no fluorine radicals are produced. The OES results show that there is a higher concentration of fluorine within the $\mathrm{SF}_{6}$ plasma than the $\mathrm{CF}_{4}$ plasma over all conditions, even though the actual flow rate is $20 \mathrm{sccm}$ for the $\mathrm{SF}_{6}$ gas and $40 \mathrm{sccm}$ of $\mathrm{CF}_{4}$. The fluorine pressure increases by $7.32 \times 10^{-4} \mathrm{~Pa}$ in the $\mathrm{SF}_{6}$ plasma and $1.31 \times 10^{-3} \mathrm{~Pa}$ in the $\mathrm{CF}_{4}$ plasma, as the bias voltage is increased from -200 to -350 and $-400 \mathrm{~V}$, respectively. The ion current density $\left(I_{i}\right)$, as a function of bias voltage is shown in Fig. 2(b). The results at $-0 \mathrm{~V}$ bias again refer to the flow of the process gases whereby the ion current density in the reactor falls to zero. The ion current density is seen to be higher in the $\mathrm{SF}_{6}$ plasma at $-200 \mathrm{~V}$, although at $-300 \mathrm{~V}$ and above the $\mathrm{CF}_{4}$ plasma produces a higher ion current density by approximately $0.01 \mathrm{~mA} / \mathrm{cm}^{2}$. The difference in $I_{\mathrm{i}}$ at higher voltages is small compared to

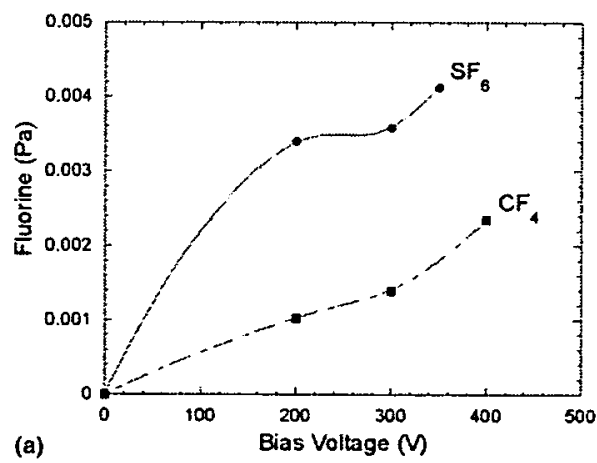

the difference when the reactor was held at $-200 \mathrm{~V}$, at which the $I_{\mathrm{i}}$ in $\mathrm{SF}_{6}$ was $0.03 \mathrm{~mA} / \mathrm{cm}^{2}$ greater than in the $\mathrm{CF}_{4}$ plasma. As the bias voltage increases, the overall $I_{i}$ increases for both plasma compositions, although the $\mathrm{SF}_{6}$ plasma shows less bias voltage dependence.

The ratio of F/C attached to the SWNTs as a function of bias voltage is shown, Fig. 3(a), from the XPS data collected 4 weeks after the functionalisation procedure. The $\mathrm{F} / \mathrm{C}$ ratio is higher in the $\mathrm{SF}_{6}$ exposed SWNTs at low bias voltages although it appears to saturate at $-300 \mathrm{~V}$, after which there is little increase in $l_{\mathrm{i}}$. The functionalisation process used here is sensitive to the reactor conditions, and there is a required balance between the amount of fluorine available and the ion current density to allow for successful attachment. The increase in the $\mathrm{F} / \mathrm{C}$ ratio seems to depend both on the ion current density increase and the increase in fluorine pressure. The $\mathrm{CF}_{4}$ exposed SWNTs show the highest F/C ratio of all at -400 $\mathrm{V}$ at which point $I_{\mathrm{i}}$ and F-pressure have both increased. However, the F-pressure is ultimately higher in the $\mathrm{SF}_{6}$ plasma leading to the conclusion that the $\mathrm{F} / \mathrm{C}$ ratio shows more of a dependence on $I_{\mathrm{i}}$. The binding energy of the $\mathrm{C}-\mathrm{F}$ bonds, going from semi-ionic to covalent in the Fls peak, is thought to depend primarily on the amount of fluorine available, whereby with higher fluorine pressure in the $\mathrm{SF}_{6}$ plasma has resulted in a more covalent $\mathrm{C}-\mathrm{F}$ bond over all reactor conditions. In

Fig. 2. As a function of bias voltage for the $\mathrm{SF}_{6}(20 \mathrm{sccm})$ and the $\mathrm{CF}_{4}(40 \mathrm{sccm})$ plasma: (a) the fluorine pressure; (b) the ion current density. The connecting line is provided for clarity.

Figure A.6: Reprint of Microelectronic engineering vol 73-74, p580 

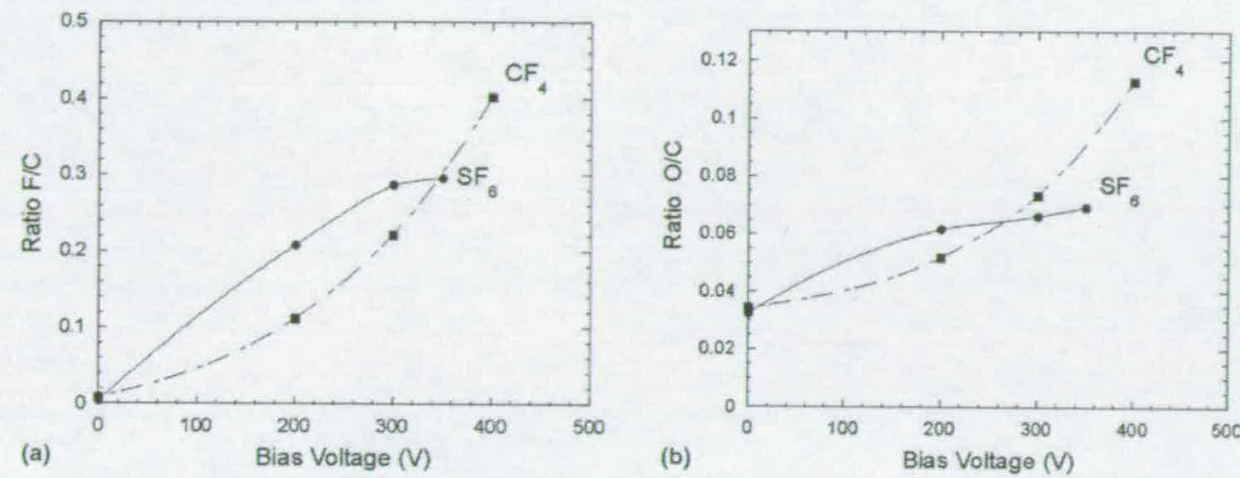

Fig. 3. As a function of bias voltage for the $\mathrm{SF}_{6}(20 \mathrm{sccm})$ and the $\mathrm{CF}_{4}(40 \mathrm{sccm})$ plasma exposed SWNTs: (a) the F/C ratio; (b) the O/ $\mathrm{C}$ ratio. The connecting line is provided for clarity.

previous work [7] it was observed that within a $\mathrm{CF}_{4}$ plasma, the intensity ratio of $I_{\text {semi-ionic }} / I_{\text {covalent }}$ in the Fls peak decreased with an increase in the $\mathrm{CF}_{4}$ gas flow. However, the F-pressure in the $\mathrm{CF}_{4}$ plasma never reaches the F-pressure achieved in the $\mathrm{SF}_{6}$ plasma over all reactor conditions, thus requiring a semi-ionic and covalent $\mathrm{Fls}$ peak component.

The percentage of oxygen attached to the SWNTs is also investigated by XPS, Fig. 3(b) and again the results taken 4 weeks after functionalisation are shown. The oxygen present here is due to contamination from the atmosphere. It is ob- served that the oxygen to carbon ratios, are higher in the $\mathrm{CF}_{4}$ plasma exposed $\mathrm{CNTs}$ and reach a saturation, similar to that observed for the $\mathrm{F} / \mathrm{C}$ in the $\mathrm{SF}_{6}$ plasma exposed CNTs. It is proposed that the oxygen is bonded to the SWNTs via surface attachment on ion induced defect sites as the oxygen ratio increases with bias voltage. The tubes exposed at $-0 \mathrm{~V}$ and the control sample, display oxygen due to defect sites already present on the SWNTs after manufacturing as well as to the bucky paper preparation process. The Fls spectra have been observed to remain unchanged with time, with the binding energies constant and hence
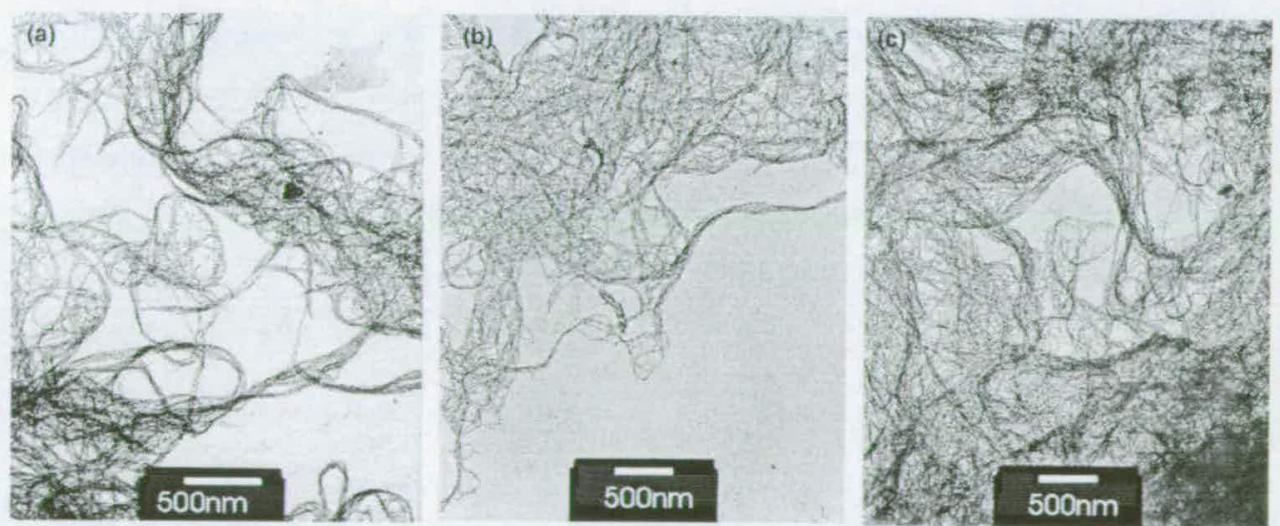

Fig. 4. TEM images of samples, at $100 \mathrm{kV}$ : (a) unexposed control samples; (b) exposed to $\mathrm{SF}_{6}$ at $-200 \mathrm{~V}$ bias; (c) exposed to $\mathrm{CF}_{4}$ at $-200 \mathrm{~V}$ bias.

Figure A.7: Reprint of Microelectronic engineering vol 73-74, p581 
it is concluded that the fluorine is well attached to the nanotubes. It is interesting to note that for all SWNTs exposed to a plasma environment, the atomic percentage of oxygen increased over time. It is likely that oxygen from the atmosphere has bonded to the SWNTs via defect sites created by the ion bombardment during the plasma exposure more so for the $\mathrm{CF}_{4}$ exposed tubes than the $\mathrm{SF}_{6}$ exposed, in agreement with the higher $I_{i}$ present in the $\mathrm{CF}_{4}$ plasma. It is noted that there is no possibility of further F-attachment as no further fluorine exposure has taken place, and the atmosphere would be unable to produce such an effect. However, it may be the case that for the $\mathrm{SF}_{6}$ exposed SWNTs, which were exposed to a higher $\mathbf{F}$ pressure throughout the scan, the fluorine attached to defect sites within the plasma, limiting the remaining regions for atmospheric oxygen to attach.

Although it is presumed that the ion bombardment within the plasma will weaken or produce defects in the SWNTs structure, the F/C ratios have not reached the saturation stoichiometry which would result in complete loss of tube structure. TEM images of the SWNTs, before and after exposure to the plasma are shown in Fig. 4(a)-(c). All three images shown are seen to be similar, with the SWNTs forming bundles due to van der Waals interactions, and the nanotubes appear to be intact. This has also been confirmed with Raman spectroscopy (not shown here), whereby the signal showed strong radial breathing modes and a large G-band relative to D-band.

\section{Conclusion}

The functionalisation of SWNTs in a plasma environment has been carried out using both a $\mathrm{CF}_{4}$ and $\mathrm{SF}_{6}$ plasma. The actinometry and ion current density results have shown that in a plasma environment with a greater atomic fluorine pressure, a more covalent $\mathrm{C}-\mathrm{F}$ attachment is observed after deconvolution of the Fls spectra, as was the case for the $\mathrm{SF}_{6}$ plasma in comparison to the $\mathrm{CF}_{4}$ plasma over all bias conditions. $\mathrm{A}$ bias voltage is required for fluorine to attach to the SWNTs. The F/C ratio is influenced more by the $I_{i}$ within the plasma rather than the F-pressure. The $\mathrm{O} / \mathrm{C}$ ratio is seen to depend on $I_{i}$ and has increased over time. It is concluded that the ion bombardment within the plasma allows sufficient energy for the C-C bonds to be weakened or even broken, hence allowing for the attachment of fluorine in the plasma system. However, the ion bombardment has not resulted in loss of nanotube structure. When there has been insufficient fluorine to attach to all the weakened bonds or defect sites atmospheric oxygen has been able to attach to the SWNTs. The fluorine attachment is seen to remain stable over time, showing that the functionalisation process is a reliable means of fluorinating SWNTs.

\section{References}

[1] Hongjie Dai, Surf. Sci. 500 (2002) 218-241

[2] Zhongfan Liu, Ziyong Shen, Tao Zhu, Shifeng Hou, Lizhen Ying, Langmuir 16 (2000) 3569-3573.

[3] Kay Hyeok An, Jeong Goo Heo, Kwan Goo Jeon, Dong Jae Bae, Chulsu Jo, Cheol Woong Yang, Chong-Yun Park, Young Hee Lee, Young Seak Lee, Young Su Chung, Appl. Phys. Lett. 80 (22) (2002) 4235-4237.

[4] Pierre R. Marcoux, Joachim Schriber, Patrick Batail, Serge Lefrant, Joel Renouard, Guy Jacob, David Albertini, Jean-Yves Mevellec, Phys. Chem. Chem. Phys. 4 (2002) 2278-2285.

[5] Bishun N. Khare, M. Meyyappan, Alan M. Cassell, Cattien V. Nguyen, Jie Han, Nano Lett. 2 (1) (2002) 73-77.

[6] Bishun N. Khare, M. Mcyyappan, Joel Kralj, Patrick Wilhite, Metagus Sisay, Hiroshi Imanaka, Jessica Koehne, Charles W. Baushchilcher Jr., Appl. Phys. Let1. 81 (27) (2002) $5237-5239$.

[7] N.O.V. Plank, Liudi Jiang, R. Chcung, Appl. Phys. Lett. 83 (12) (2003) 2426-2428.

[8] G. Nanse, E. Papirer, P. Fioux, F. Moguet, A. Tressaud, Carbon 35 (2) (1997) 175-194.

[9] John F. Moulder, William F. Stickle, Peter E. Sobol, Kenneth D. Bomben, Handbook of X-ray Photoelectron Spectroscopy, Perkin-Elmer Corporation, 1993.

[10] V.M. Donelly, D.L. Flamm, W.C. Dautermontsmith, D.J. Werder, J. Appl. Phys. 55 (1984) 242.

Figure A.8: Reprint of Microelectronic engineering vol 73-74, p582 
HTML AESTP.ACT - LIMKS

APPLIED PHYSICS LETTERS

VOLUME 85, NUMBER IS

11 OCTOBER 2004

\title{
Thiolation of single-wall carbon nanotubes and their self-assembly
}

\author{
N. O. V. Plank ${ }^{\text {a) }}$ and R. Cheung \\ School of Engineering and Electronics, University of Edinburgh, Sconish Microelectronics Centre \\ West Mains Roal. King's Buildings. EH9 3JF, Scotland, Uniled Kingdom \\ R. J. Andrews \\ School of Chemistry, Universiry of Edinburgh, Joseph Black Building, West Mains Road, King's Buildings, \\ EH9 3JL. Scotland, United Kingdom \\ (Received 12 May 2004; accepted 10 August 2004) \\ A method for the thiolation of single-wall carbon nanotubes has been developed by exposing a \\ sulfur/carbon nanotubes mixture to an argon/hydrogen gaseous plasma. X-ray photoelectron \\ spectroscopy provides evidence of the existence of sulfur attached to carbon on the carbon \\ nanotubes samples and Raman spectroscopy results show that the carbon nanotubes" structure has \\ been preserved after the treatment. One added advantage of the reported method is that excess \\ oxygen is not present on the nanotubes. The thiolated carbon nanotubes are shown to self-assemble \\ onto gold electrodes. Our method for thiolating carbon nanotubes provides a reliable and simple \\ way for preparing functionalized tubes for nanoelectronic circuits based on carbon nanotubes. \\ (1) 2004 American Institute of Physics. [DOI: 10.1063/1.1805702]
}

Carbon nanotubes (CNTs) offer the electronic device community a promising candidate for future electronic needs. The nanotubes themselves have shown excellent conduction properties with the ability to behave as semiconducting or metallic conducting tubes depending on how the tubes have formed. ${ }^{1.2}$ The inherently small size of the nanotubes also allow for instant nanometer scale device production. ${ }^{3}$ However, there are problems remaining in the field regarding how to selectively position the tubes for accurate, reproducible and high volume device production. One method to enable selective positioning for the production of CNT devices is to functionalize the nanotubes. Thiols are known to bond selectively to gold and other reports have demonstrated thiol functionalization techniques using long and short chain alkanethiols respectively. ${ }^{4-6}$ Here, we report on the direct thiolation of single-walled CNTs, without any long chain carbon groups, by first physically mixing elemental sulfur with CNTs and then exposing the sulfur/CNT mixture to an $\mathrm{Ar} / \mathrm{H}_{2}$ plasma. The advantage of this method has been to allow CNTs to attach selectively to gold electrodes with a short functionalization time.

Single-wall CNTs from Carbon Nanotechnologies Inc. were used in our experiments. The sulfur/CNTs were prepared by physical mixing with elemental sulfur from Aldrich. The nontreated sample, sample $I$, is referred to as the control sample throughout the text. Other samples were exposed to an argon and hydrogen based plasma mixture within a plasma etch reactor with the electrodes heated to $120^{\circ} \mathrm{C}$ to allow the sulfur to melt, the reactor conditions and mixing ratios are outlined in Table I. After, the control CNT sample, nonplasma exposed and plasma exposed sulfur/CNT samples were immediately placed in $2 \mathrm{ml}$ of toluene and rinsed through with $5 \mathrm{ml}$ of toluene to rid of any excess sulfur before being drop coated onto a silicon wafer for characterization. For the self-assembly experiments, a drop of the functionalized nanotubes solution was placed in $2 \mathrm{ml}$ of ethanol and sonicated for $10 \mathrm{~min}$. A lithographically patterned

"Electronic mail: natalie.plank@ecc.ed.ac.uk clean gold sample was then left in the solution and covered for $12 \mathrm{~h}$, after which the sample was cleaned in ethanol and viewed in the Hitachi $\$ 4500$ scanning electron microscope. To characterize the extent of sulfur attachment to the nanocubes before self-assembly, x-ray photoelectron spectroscopy (XPS) and Raman spectroscopy have been used. In addition. we demonstrate the self-assembly of the thiolated tubes onto patterned gold electrodes.

Figure 1 shows the $\mathrm{S} 2 p$ peaks for the control CNTs, CNTs that have been mixed with sulfur (sulfur/CNTs) and sulfur/CNTs mixture exposed to $\mathrm{Ar} / \mathrm{H}_{2}$ plasma. The peaks have been fitted using XPS peak software, with $\Delta E$ $=1.18 \mathrm{eV}$ between the $S p_{3 / 2}$ and $S p_{1 / 2}$ and fixed intensity ratio of 2:1 respectively. A mixture of Lorentz:Gaussian peaks were required at $70: 30$, with full width at half maximum of 1 as in the literature. ${ }^{7.8}$ It has been observed in our experiments, that for all CNT samples exposed to sulfur treatment, a binding energy of $163.7-163.8 \mathrm{eV}$ is found. According to the literature, $\mathrm{S}-\mathrm{H}$ is present at $163.7 \mathrm{eV}$ while $\mathrm{S}-\mathrm{S}$ occurs at $163.8 \mathrm{eV}$ and $\mathrm{S}-\mathrm{Au}$ bond is known to occur at $162 \mathrm{eV}^{7-9}$ Multilayer thiols have also been observed at $164.4 \mathrm{cV} .^{10}$ Due to the closeness of the energy peaks for sulfur-containing compounds, we conclude that some form of sulfur is bonded to the CNTs after sulfur treatment within the thiol region.

Figure 2 shows the influence of the flow rate of $\mathrm{H}_{2}$ in the $\mathrm{Ar} / \mathrm{H}_{2}$ gas nixture during plasma exposure on (a) the S/C ratio and (b) the position of the $S 2 p$ energy peak. The amount of S/C (around 5\%) does not appear to be affected

TABLE I. The weight of cartom nanotubes and sulfur and the neactor cunditions, if power was held at $250 \mathrm{~W}$ and exposure time was $60 \mathrm{~s}$ throughout.

\begin{tabular}{llll}
\hline Sample & Weight of sulfur/C.NT (g) & Ar flow (scem) & $\mathrm{H}_{2}$ fow (scem)
\end{tabular}

\begin{tabular}{cccc}
\hline Control & $0 / 0.002$ & No plasma & No plasma \\
1 & $0.02 / 0.002$ & No plasma & No plasma \\
2 & $0.02 / 0.002$ & 75 & 25 \\
3 & $0.02 / 0.002$ & 50 & 50 \\
4 & $0.02 / 0.002$ & 25 & 75 \\
\hline \hline
\end{tabular}

$0003-6951 / 2004 / 85(15) / 3229 / 3 / \$ 22.00$

Figure A.9: Reprint of APL vol 85, p3229 


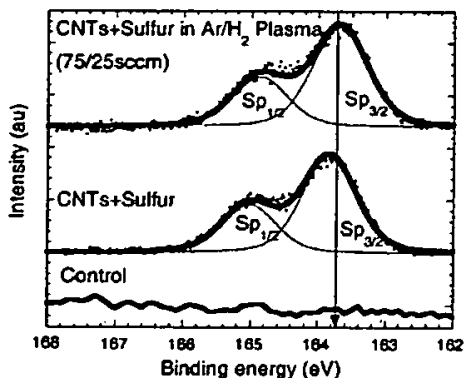

FIG. I. X-ray photoelectron spectra of $S 2 p$ peak for control CNTs, sulfur/ CNTs, and sulfur/CN'Ts exposed to $\mathrm{Ar} / \mathrm{H}_{2}$ plasma.

significantly by the presence of the plasma or by the rate of $\mathrm{H}_{2}$ flow, yet the presence of the plasma does shift the $\mathrm{S} 2 p$ peaks to slightly lower binding energies. Therefore, we see that the exposure to the $\mathrm{Ar} / \mathrm{H}_{2}$ plasma is not essential for sulfur to atlach to the CNTs but does help to provide an excess $\mathrm{H}_{2}$ environment for thiolation to take place. Plasma functionalization of graphite and glassy carbon using $\mathrm{SO}_{2}$ plasma have shown similar sulfur atomic percentages and position of the $S 2 p$ peaks, at dc bias voltages and exposure times close to those used in our experiments. "Moreover, the adhesion of sulfur in the thiol region without the presence of additional $\mathrm{S}-\mathrm{O}$ peaks is demonstrated in our thiolation method, even after 4 weeks exposure to atmosphere. The $\mathrm{SO}_{2}$ bonding, often found in different methods of treating the tubes ${ }^{4.5}$ and other forms of carbon, ${ }^{6,12.13}$ is observed by others usually at $\sim 167 \mathrm{eV}$. In addition, the oxygen peak com* ponent has not been seen to increase in our thiolated tubes after 4 wecks exposure to atmosphere, as has been the case in our previous studies on the fluorine functionalization process. ${ }^{14.15}$ Experimental evidence suggests that the lack of oxygen incorporation into the tubes is inherent to our thiolization process.

The Raman spectra for the control CNTs, sulfur/CNTs, and sulfur/CNTs exposed to $\mathrm{Ar} / \mathrm{H}_{2}$ plasma are shown in Fig. 3. The presence of $D$ and $G$ bands and the RBM modes indicate that the tubular structure of the nanotubes have not been destroyed by our treatments, Fig. 3(a), and that the average tube diameter distribution ranges from 0.8 to $1.3 \mathrm{~nm}^{16}$ Many low intensity peaks inherent to the Raman

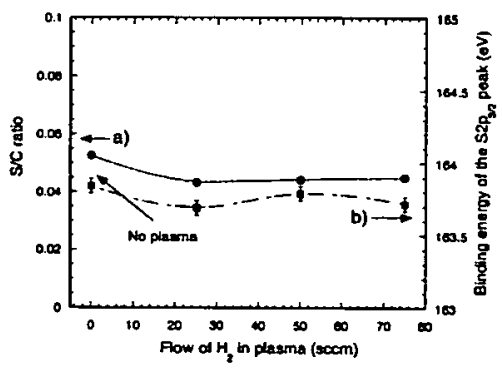

FIG. 2. Influence of $\mathrm{H}_{2}$ flow rate in the $\mathrm{Ar} / \mathrm{H}_{2}$ plasma exposure (total flow $=100 \mathrm{secm}$ ) on (a) the $\mathrm{S} / \mathrm{C}$ matio and (b) the pasition of the $S 2 p$ energy $=100 \mathrm{sccm})$ on (a) the $\mathrm{S} / \mathrm{C}$ ratio and (b) the pasition of the $S 2 p \mathrm{enc}$
peak, of the sulfur/CNTs. At $H_{3}=0 \mathrm{sccm}$, no plasma has been struck.
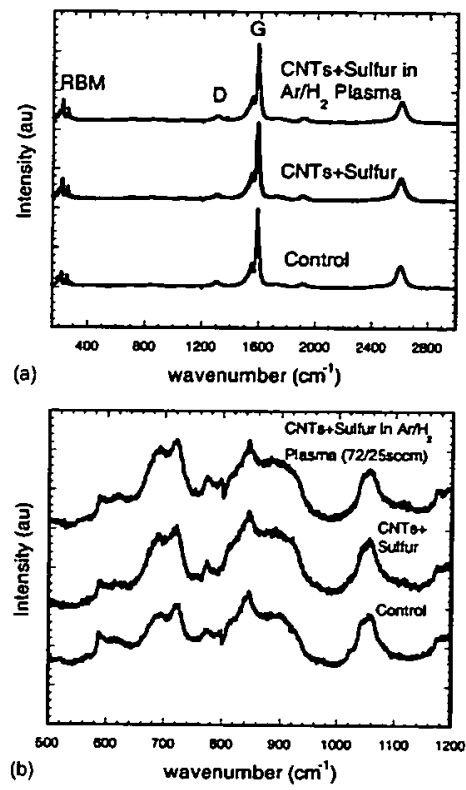

FIG. 3. Raman spectro of control CNTs, sulfur/CNTs, and sulfur/CNTs exposed to $\mathrm{Ar} / \mathrm{H}_{2}$ plasma, (a) over the whule spectra and (b) zoomed in over $500-1200 \mathrm{~cm}^{-1}$.

spectra of carbon nanotubes ${ }^{17,18}$ are evident in all three samples, Fig. 3(b). Neither the $S=C$ peak at $1095 \mathrm{~cm}^{-1}$ nor $S-S$ peak at $585 \mathrm{~cm}^{-1}$ have been observed after our thiolization treatment, in contrast to results reported by Liu et al. ${ }^{5}$ The lack of $S$ as indicated by Raman in our experiments could be due to the small sulfur atomic concentration measured, around $5 \%$ by XPS experiments, whereas in the method used by Liu et al. the sulfur content is expected to be higher. Other work where carbon films and carbon rods have been doped with sulfur resulting in atomic percentages similar to those observed in our experiments also show no sulfurrelated peaks in the Raman spectra. ${ }^{19-23}$

We have used the thiolated tubes in the self-assembly process and Fig. 4 shows both low and high resolution scanning electron micrographs of the functionalized tubes lying across the gold electrodes after self-assembly. The success of the self-assembly indicates the existence of thiols in our tubes. The CNT bundles are seen to align across the electrodes as opposed to along the electrodes, probably due to the sulfur bonding preferentially to the ends of the nanotubes. Several authors have indicated the end groups of CNTs to be the most reactive part of the tubes. ${ }^{1.2,4.3}$ The low stochiometries shown in Fig. 2 support the hypothesis that the sulfur will not have bonded along the entire length of the CNTs. In comparison, the sulfur/CNT nonplasma exposed sample has shown no sign of self-assembly to the gold electrodes or any surface physisorption effects after the selfassembly stage.

Thiolation of single-walled CNTs has been demonstrated whereby the ends of the nanotube bundles have been selectively functionalized making it favorable for the nanotubes to

Figure A.10: Reprint of APL vol 85, p3230 


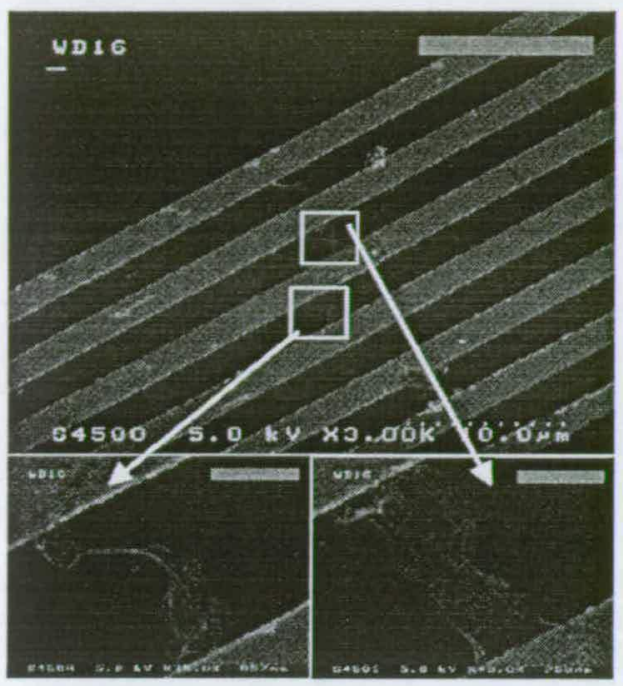

FIG. 4. Scanning electron micrographs for self-sssembled thiolated tubes onto gold electrodes. The tubes shown are from kample 2.

self-assemble across the gold electrodes. Similarly prepared carbon nanotube films have been assembled over gold electrodes on $\mathrm{SiO}_{2}$ and have shown an average resistance of $15454 \Omega$ at $10 \mathrm{~V}$ (Fig. 5). The current is higher than that observed for single CNTs bridging electrodes by electric field alignment methods. ${ }^{24}$ Work is currently under way to measure the electrical properties of the connected tubes after selective alignment of the tubes specific for nanoelectronic circuit fabrication.

The authors would like to thank Ronald Brown for assistance in the XPS measurements, Gavin Whittaker for use of the Plasma Etch reactor and discussions, Sara Falconi for use of the Raman spectroscopy equipment and Andrew Alexander.

R. Saito, G. Dressethaus, and M. S. Dresselhaus, Physical Properties of Carbon Nanotubes (Imperial College Press, London, 2001)

${ }^{2}$ H. Dai, Surf. Sci. 500, $218(2002)$.

'S. J. Tans, A. R. M. Verschueren, and C, Dekker, Nature (London) 393 , 49 (1998)

'Z. Liu, Z. Shen, T. Zhu, S. Hou, and L. Ying, Langmuir 16, $3569(2000)$. 5. K. Lim, W. S. Yun. M. -h. Yoon, S. K. Lee, C. H. Kim, K. Kim, and S.

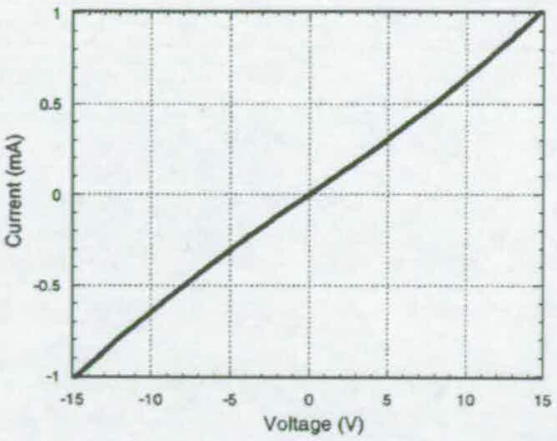

FIG. 5. The average current-voltage characteristics at room temperature of a CNT film produced onto gold electrodes after sulfur plasma exposure.

K. Kim, Synth. Met. 139, 521 (2003)

${ }^{6} \mathrm{M}$. Zharnikov and M. Grunze, J. Phys.: Condens, Matter 13, 11333 (2001).

${ }^{2}$ Y. Joseph, N. Krasteva, L Besnard, B. Guse, M. Rusenherger, U. With A. Knop-Gerick Knop-Gericke, R. Schlogl, R. Krustev, A. Yasuda, and T. Vossmeyer. Faraday Discuss. 125, 77 (2003)

C. Vericat, M. E. Vela, G. Andreasen, and R. C. Salvarezza, Langmuir 17, $4919(2001)$.

C. M. Whelan, M. R. Smyth. C. J. Barnes, N. M. D. Brown, and C. A. Anderson, Appl. Surf. Sci. 134, 144 (1998).

${ }^{10}$ S. M. Kane and J. L. Gland, Surf. Sci. 468. $101(2000)$

"M. Collaud Coen, B, Keller, P. Groening, and L. Schlaphach, J. Apph. Phys, 92, 5077 (2002)

12. J. Heeg, C. Kramer, M. Wolter, S. Michaclis, W. Plicth, and W. -J. Fischer, Appl. Surf. Sci. 180, $36(2001)$.

13., C. Love, D. B. Wolfe, R. Haasch, M. L. Chabinyc, K. E. Paul, G. M. Whitesides, and R. G. Nuzzo, J. Am. Chem. Soc, 125, $2597(2003)$

${ }^{14} \mathrm{~N}$. O. V. Plank, L. Jiang, and R. Cheung, Appl. Phys. Lett. 83, 2426 (2003)

(2003). P. Plank and R. Cheung. Microclectron. I

${ }^{16}$ M. S. Dresselhaus, G. Dresselhaus, A. Jorio, A. G. Souza Filho, M. A. Pimenta, and R. Saito, Acc. Chem. Res. 35, 1070 (2002).

"H. Kuzmany, B. Burger, A. Thess, and R. E. Smalley, Carbon 36, 709 (1998)

A. M. Rao, E. Richter, S. Bandow, B. Chase, P. C. Eklund, K. A. Will iams, S. Fang, K. R. Subbaswamy, M. Menon, A. Thess, R. E. Smalley, G. Dresselhaus, and M. S. Dresselhaus, Science 275, 187 (1997).

${ }^{19}$ A. Gonzalez-Berrios, D. Huang, N. M. Medina-Emmanuelli, K. E. Kristian, O. O Ortiz I A. Gonzalez I. De lesus, L. Y Vargas, B. R. Weiner. and G. Morell, Diamond Relat. Mater. 13, 221 (2004).

. $86.4+64(1900)$

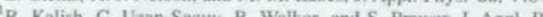
3923 (2003).

${ }^{2}$ S. Gupta, B. R. Weiner, and G. Morell, J. Appl. Phys, 91, $10088(2002)$

${ }^{2}$ S. Gupta, B. R. Weiner, and G. Morell, J. Appl. Phys. 92, 5457 (2002).

${ }^{24}$ M. S. Kumar, T. H. Kim, S. H. Lee, S. M. Song, J. W. Yang, K. S. Nahrm, and E.-K. Suh, Chem. Phys. Lett. 383, 235 (2004)

Figure A.11: Reprint of APL vol 85, p3231 


\section{References}

[1] "http://www.intel.com/technology/mooreslaw/index.html."

[2] R. Saito, G. Dresselhaus, and M. S. Dresselhaus, Physical Properties of Carbon Nanotubes. Imperial College Press, 2001.

[3] "Carbon nanotubes: special edition," Physics World, vol. 13(6), pp. 31-53, June 2000.

[4] "Nanoelectronics: On the tube," The Economist, May 10, 2003.

[5] F. Davey, D. Firn, and P. Smith, "Will nanotech become the next biotech bubble?," The Financial Times, p. 13, March 19, 2003.

[6] M. Fettes, "Science's new big idea means thinking small," Edinburgh Evening News, p. 14, June 24, 2003.

[7] "http://www.cnanotech.com."

[8] V. N. Khabashsku, W. E. Billups, and J. L. Margrave, "Fluorination of singlewalled carbon nanotubes and subsequent derivatization reactions," Accounts of Chemical Research, vol. 35, no. 12, pp. 1087-1095, 2002.

[9] P. R. Marcoux, J. Schrieber, P. Batail, S. Lefrant, J. Renouard, G. Jacob, D. Albertin, and J.-Y. Mevellec, "A spectroscopic study of the fluorination and defluorination reactions on single-walled carbon nanotubes," Physical chemistry and chemical Physics, vol. 4, pp. 2278-2285, 2002.

[10] E. T. Mickelson, C. B. Huffman, A. G. Rinzler, R. E. Smalley, R. H. Hauge, and J. L. Margrave, "Fluorination of single-wall carbon nanotubes," Chemical Physics Letters, vol. 296, pp. 188-194, 1998.

[11] L. Valentini, D. Puglia, I. Armentano, and J. M. Kenny, "Sidewall functionalization of single-walled carbon nanotubes through $\mathrm{CF}_{4}$ plasma treatment and subsequent reaction with aliphatic amines," Chemical Physics Letters, vol. 403, pp. 385-389, 2005.

[12] L. Valentini, I. Armentano, F. Mengoni, D. Puglia, G.Pennelli, and J. M. Kenny, "Chemical gating and photoconductivity of $\mathrm{CF}_{4}$ plasma-functionalized singlewalled carbon nanotubes with adsorbed butylamine," Journal of Applied Physics, vol. 97, p. 114320, 2005.

[13] Y. W. Zhu, F. C. Cheong, T. Yu, X. J. Xu, C. T. Lim, J. T. L. Thong, Z. X. Shen, C. K. Ong, Y. J. Liu, A. T. S. Wee, and C. H. Sow, "Effcets of $\mathrm{CF}_{4}$ plasma on the field emission properties of aligned multi-wall carbon nanotube films," Carbon, vol. 43, pp. 395-400, 2005. 
[14] Z. N. Utegulov, D. B. Mast, P. He, D. Shi, and R. F. Gilland, "Functionalization of single-walled carbon nanotubes using isotropic plamsa treatment: Resonant raman spectroscopy study," Journal of Applied Physics, vol. 97, p. 104324, 2005.

[15] M. Shim, A. Javey, N. W. S. Kam, and H. Dai, "Polymer functionalization for airstable n-type carbon nanotube field-effect transistors," Journal of the American Chemical Society, vol. 123, pp. 11512-11513, 2001.

[16] J. L. Stevens, A. Y. Huang, H. Peng, I. W. Chiang, V. N. Khabashesku, and J. L. Margrace, "Sidewall amino-functionalization of single-walled carbon nanotubes through fluorination and subsequent reactions with terminal diamines," Nano Letters, vol. 3, pp. 331-336, 2003.

[17] N. O. V. Plank, L. Jiang, and R. Cheung, "Fluorination of carbon nanotubes in $\mathrm{CF}_{4}$ plasma," Applied Physics Letters, vol. 83, pp. 2426-2428, 2003.

[18] N. O. V. Plank and R. Chenug, "Functionalisation of carbon nanotubes for molecular electronics," Microelectronic Engineering, vol. 73-74, pp. 578-582, 2004.

[19] Y. Zhang, A. Chang, J. Chao, Q. Wang, W. Kim, Y. Li, N. Morris, E. Yenilmez, J. Kong, and H. Dai, "Electric-field-directed growth of aligned single-walled carbon nanotubes," Applied Physics Letters, vol. 79, pp. 3155-3157, 2001.

[20] J. Li, Q. Ye, A. Cassell, H. T. Ng, R. Stevens, J. Han, and M. Meyyappan, "Bottum-up approach for carbon nanotube interconnnects," Applied Physics Letters, vol. 82, pp. 2491-2493, 2003.

[21] J. Kong, H. T. Soh, A. M. Cassell, C. F. Quate, and H. Dai, "Synthesis of individual single-walled carbon nanotubes on patterned silicon wafers," Nature, vol. 395, pp. 878-881, 1998.

[22] B. Q. Wei, R. Vajtai, Y. Jung, J. Ward, R. Zhang, G. Ramanath, and P. M. Ajayan, "Organized assembley of carbon nanotubes," Nature, vol. 416, pp. 495-496, 2002.

[23] E. Joselevich and C. M. Lieber, "Vectorial growth of metallic and semiconducting single-wall carbon nanotubes," Nano Letters, vol. 2, pp. 1137-1141, 2002.

[24] J. Kong, N. R. Franklin, C. Zhou, M. G. Chapline, S. Peng, K. Cho, and H. Dai, "Nanotube molecular wires as chemical sensors," Science, vol. 287, pp. 622-625, 2000.

[25] Y. Zhang and H. Dai, "Formation of metal nanowires on suspended single-walled carbon nanotubes," Applied Physics Letters, vol. 77, no. 19, pp. 3015-3017, 2000.

[26] S. Fan, M. G. Chaplin, N. R. Franklin, T. W. Tombler, A. M. Cassell, and H. Dai, "Self-orientated regular arrays of carbon nanotubes and their field emission properties," Science, vol. 283, pp. 512-514, 1999.

[27] P. L. McEuen, "Single-all carbon nanotubes," Physics World, vol. 13(6), pp. 31$36,2000$. 
[28] S. Li, Z. Yu, S.-F. Yen, W. C. Tang, and P. J. Burke, "Carbon nanotube transistor operation at 2.6 GHz," Nano Letters, vol. 4, pp. 753-756, 2004.

[29] J. A. Misewich, R. Martel, P. Avouris, J. C. Tsang, S. Heinze, and J. Tersoff, "Electrically induced optical emission form a carbon nanotube FET," Science, vol. 300, pp. 783-786, 2003.

[30] C. Zhou, J. Kong, and H. Dai, "Electrical measurements of individual semiconducting single-walled carbon nanotubes of various diameters," Applied Physics Letters, vol. 76, no. 12, pp. 1597-1599, 2000.

[31] H. Dai, "Carbon nanotubes: opportunities and challenges," Surface Science, vol. 500, pp. 218-241, 2002.

[32] M. S. Dresselhaus, G. Dresselhaus, A. Jorio, A. G. S. Filho, M. A. Pimenta, and R. Saito, "Single nanotube raman spectroscopy," Accounts of Chemical Research, vol. 35, pp. 1070-1078, 2002.

[33] J. Chen, M. A. Hamon, H. Hu, Y. Chen, A. M. Rao, P. C. Eklund, and R. C. Haddon, "Solution properties of single-walled carbon nanotubes," Science, vol. 282, pp. 95-98, 1998.

[34] Z. Liu, Z. Shen, T. Zhu, S. Hou, and L. Ying, "Organising single-walled carbon nanotubes on gold using a wet chemical self-assembling technique," Langmuir, vol. 16, pp. 3569-3573, 2000.

[35] J. K. Lim, wan Soo Yun, M. han Yoon, S. K. Lee, C. H. Kim, K. Kim, and S. K. Kim, "Selective thiolation of single-walled carbon nanotubes," Synthetic Metals, vol. 139, pp. 521-527, 2003.

[36] M. Hazani, D. Shvarts, D. Peled, V. Sidorov, and R. Naaman, "Self-assembled carbon-nanotube-based field-effect transistors," Applied Physics Letters, vol. 85, pp. 5025-5027, 2004.

[37] S. Jin, D. Whang, M. C. McAlpine, R. S. Friedman, Y. Wu, and C. M. Lieber, "Scalable interconnection and integration of nanowire devices without registration," vol. 4, pp. 915-919, 2004.

[38] S. G. Rao, L. Huang, W. Setyawan, and Seunghun, "Large-scale assembly of carbon nanotubes," Nature, vol. 425, pp. 36-37, 2003.

[39] J. Liu, A. G. Rinzler, H. dai, J. H. Hafner, R. K. Bradley, P. J. Boul, A. Lu, T. Iverson, K. Shelimov, C. B. Huffman, F. Rodriguez-Macias, Y.-S. Shon, T. R. Lee, D. T. Colbert, and R. E. Smalley, "Fullerene pipes," Science, vol. 280, pp. 1253-1256, 1998.

[40] Y. Xia and G. M. Whitesides, "Soft lithography," Angew. Chemistry International Edition, vol. 37, pp. 550-575, 1998.

[41] Y. Xia and G. M. Whitesides, "Soft lithography," Annual Review of Materials Science, vol. 28, pp. 153-184, 1998. 
[42] M. Zharnikov and M. Grunze, "Spectroscopic charcterization of thiol-derived self-assembling monolayers," Journal of Physics:Condensed Matter, vol. 13, pp. 11333-11365, 2001.

[43] N. L. Abbott, C. B. Gorman, and G. M. Whitesides, "Active control of wetting using applied electrical potentials and self-assembled monolayers," Langmuir, vol. 11, pp. 16-18, 1995.

[44] M. Lee and C. Chung, "Self-assembled monolayers of ferrocene-terminated thiol on gold: Reorientation induced by coadsorption of dodecanethiol," Bulletin of the Korean Chemical Society, vol. 20, pp. 1-3, 1999.

[45] J. B. Miller, "Amines and thiols on diamond surfaces," Surface Science, vol. 439, pp. 21-33, 1999.

[46] G. Patrick, Instant Notes: Organic Chemistry. 9 Newtec place, Magdalen Road, Oxford OX4 1RE, UK: BIOS Scientific Publishers, 2000.

[47] P. R. Wallace, “The band theory of graphite," Physical Reviews, vol. 71, pp. 622 634, 1947.

[48] C. Kittel, Introduction to solid state physics: seventh edition. New York, USA: John Wiley and Sons, 1996.

[49] J. W. Mintmire, B. I. Dunlap, and C. T. White, "Are fullerene tubes metallic?," Physical Review Letters, vol. 68, pp. 631-634, 1992.

[50] N. Hamada, S. Sawada, and A. Oshiyama, "New one-dimensional conductors: Graphitic microtubules," Physical Review Letters, vol. 68, pp. 1579-1581, 1992.

[51] R. Saito, M. Fujita, G. Dresselhaus, and M. S. Dresselhaus, "Electronic structure of chiral graphene tubes," Applied Physics Letters, vol. 60, pp. 2204-2206, 1992.

[52] R. Saito, M. Fujita, G. Dresselhaus, and M. S. Dresselhaus, "Electronic structure of graphene tubules based on $\mathrm{c}_{60}$," Physical Review B, vol. 46, pp. 1804-1811, 1992.

[53] T. Durkop, B. M. Kim, and M. S. Furher, "Properties and applications of high-mobility semiconducting nanotubes," Journal of Physics:Condensed Matter, vol. 16, pp. R553-R580, 2004.

[54] S. G. Louie, "Electronic properties, junctions and defects of carbon nanotubes," in Topics in Applied Physics: Carbon Nanotubes (M. S. Dresselhaus, G. Dresselhaus, and P. Avouris, eds.), 80, (Berlin Heidelberg 2001), pp. 113-145, Spriner-Verlag, 2001.

[55] S. J. Tans, M. H. Devoret, H. Dai, A. Thess, R. E. Smalley, L. J. Geerligs, and C. Dekker, "Individual single-wall carbon nanotubes as quantum wires," Nature, vol. 386, pp. 474-477, 1997.

[56] C. T. White and T. N. Todorov, "Carbon nanotubes as long ballistic conductors," Nature, vol. 393, pp. 240-242, 1998. 
[57] S. Frank, P. Poncharal, Z. L. Wang, and W. A. de Heer, "Carbon nanotube quantum resistors," Science, vol. 280, pp. 1744-1746, 1998.

[58] A. Javey, J. Guo, Q. Wang, M. Lundstrom, and H. Dai, "Ballistic carbon nanotube field-effect transistors," Nature, vol. 424, pp. 654-657, 2003.

[59] D. Mann, A. Javey, J. Kong, Q. Wang, and H. Dai, "Ballistic transport in metallic nanotubes with reliable Pd ohmic contacts," Nano Letters, vol. 3, pp. 1541-1544, 2003.

[60] R. Seidel, A. P. Graham, E. Unger, G. S. Duesberg, M. Liebau, W. Steinhoegl, F. Kreupl, and W. Hoenlein, "High-current nanotube transistors," Nano Letters, vol. 4, pp. 831-834, 2004.

[61] P. L. McEuen, M. Bockrath, D. H. Cobden, and J. G. Lu, "One dimensional transport in carbon nanotubes," Microelectronic Engineering, vol. 47, pp. 417-420, 1999.

[62] J. W. Park, J. Kim, J. O. Lee, K. C. Kang, J. J. Kim, and K. H. Yoo, "Effects of artificial defects on the electrical transport of single-walled carbon nanotubes," Applied Physics Letters, vol. 80, no. 1, pp. 133-135, 2002.

[63] M. Bockrath, D. H. Cobden, J. Lu, A. G. Rinzler, R. E. Smalley, L. Balents, and P. L. McEuen, "Luttinger-liquid behaviour in carbon nanotubes," Nature, vol. 397, pp. 598-601, 1999.

[64] S. J. Wind, R. M. J. Appenzeller, V. Derycke, and P. Avouris, "Vertical scaling of carbon nanotube field-effect transistors using top gate electrodes," Applied Physics Letters, vol. 80, pp. 3817-3819, 2002.

[65] R. C. Weast, ed., Handbook of Physics and Chemistry. U.S.A. : The Chemical Rubber Co., 1974.

[66] S. J. Tans, A. R. M. Verschueren, and C. Dekker, "Room-temperature transistor based on a single carbon nanotube," Nature, vol. 393, pp. 49-52, 1998.

[67] P. Avouris, R. Martel, S. Heinze, M. Radosavljevic, S. Wind, V. Derycke, J. Appenseller, and J. Terso, "The role of schottky barriers on the behaviour of carbon nanotube field effect transistors," in Structural and Electronic Properties of Molecular Nanostructures (H. Kuzmany, J. Fink, M. Mehring, and S. Roth, eds.), 633, (Kirchberg, Tirol, Austria), pp. 508-512, AIP conference Proceedings 633, 2002.

[68] J. Appenzeller, J. Knoch, V. Derycke, R. Martel, S. Wind, and P. Avouris, "Fieldmodulated carrier transport in carbon nanotube transistors," Physical Review Letters, vol. 89, pp. 126801-1-4, 2002.

[69] S. Heinze, J. Tersoff, and P. Avouris, "Electrostatic engineering of nanotube transistors for imporved performance," Applied Physics Letters, vol. 83, pp. 50385040, 2003. 
[70] S. Heinze, J. Tersoff, R. Martel, V. Derycke, J.Appenzeller, and P. Avouris, "Carbon nanotubes as schottky barrier transistors," Physical Review Letters, vol. 89, pp. 106801-1-4, 2002.

[71] J. Appenzeller, M. Radosavljevic, J. Knoch, and P. Avouris, "Tunneling versus thermionic emission in one-dimensional semiconductors," Physical Review Letters, vol. 92, pp. 048301-1-4, 2004.

[72] P. Avouris, R. Martel, V. Derycke, and J. Appenzeller, "Carbon nanotube transistors and logic circuits," Physica B, vol. 323, pp. 6-14, 2002.

[73] T. Durkop, S. A. Getty, E. Cobas, and M. S. Fuhrer, "Extraordinary mobility in semiconducting carbon nanotubes," Nano Letters, vol. 4, pp. 35-39, 2003.

[74] P. G. Collins, M. S. Arnold, and P. Avouris, "Engineering carbon nanotubes and nanotube circuits using electrical breakdown," Science, vol. 292, pp. 706-709, 2001.

[75] A. Javey, Q. Wang, A. Ural, Y. Li, and H. Dai, "Carbon nanotube transistor arrays for multistage complementary logic and ring oscillators," Nano Letters, vol. 2, pp. 929-932, 2002.

[76] J. U. Lee, P. P. Gipp, and C. M. Heller, "Carbon nanotube $p$ - $n$ junction diodes," Applied Physics Letters, vol. 85, pp. 145-147, 2004.

[77] R. Krupke, F. Hennrich, H. v. Lohneysen, and M. M. Kappes, "Separation of metallic from semiconducting single-walled carbon nanotubes," Science, vol. 301, pp. 344-347, 2003.

[78] K. Balasubramanian, R. Sordan, M. Burghard, and K. Kern, "A selective electrochemical approach to carbon nanotube field-effect transistors," Nano Letters, vol. 4, pp. 827-830, 2004.

[79] J. Cui, M. Burghard, and K. Kern, "Reversible sidewall osmylation of individual carbon nanotubes," Nano Letters, vol. 3, pp. 613-615, 2003.

[80] R. Krupke, F. Hennrich, H. B. Weber, M. M. Kappes, and H. v. Lohneysen, "Simulataneous deposition of metallic bundles of single-walled carbon nanotubes using AC-dieletrophoresis," Nano Letters, vol. 3, pp. 1019-1023, 2003.

[81] J. Chung, K.-H. Lee, J. Lee, and R. S. Ruoff, "Toward large-scale integration of carbon nanotubes," Langmuir, vol. 20, pp. 3011-3017, 2004.

[82] M. S. Kumar, T. H. Kim, S. H. Lee, S. M. Song, J. W. Yang, K. S. Nahm, and E.K. Suh, "Influence of electric field type on the assembly of single walled carbon nanotubes," Chemical Physics Letters, vol. 383, pp. 235-239, 2004.

[83] K. Yamamoto, S. Akita, and Y. Nakayama, "Orientation of carbon nanotubes using electrophoresis," Japanese Journal of Applied Physics, vol. 7, pp. 917-918, 1996. 
[84] J. Li, Q. Zhang, D. Yang, and J. Tian, "Fabrication of carbon nanotube field effect transistors by AC dielectrophoresis method," Carbon, vol. 42, pp. 2263-2267, 2004.

[85] V. Derycke, R. Martel, J. Appenzeller, and P. Avouris, "Carbon naotube inter- and intramolecular logic gates," Nano Letters, vol. 1, no. 9, pp. 453-456, 2001.

[86] V. Derycke, R. Martel, J. Appenzeller, and P. Avouris, "Controlling doping and carrier injection in carbon nanotube transistors," Applied Physics Letters, vol. 80, no. 15, pp. 2773-2775, 2002.

[87] M. Radosavljevic, M. Freitag, K. V. Thadani, and A. T. Johnson, "Nonvolatile molecular memory elements based on ambipolar nanotube field effect transistors," Nano Letters, vol. 2, pp. 761-764, 2002.

[88] S.Kazaoui, N.Minami, R.Jacquemin, H.Kataura, and Y.Achiba, "Amphoteric doping of single-wall carbon-nanotube thin films as probed by optical absorption spectroscopy," Physical Review B, vol. 60, no. 19, pp. 13339-13342, 1999.

[89] A. M. Rao, P. C. Eklund, S. Bandow, A. Thess, and R. E. Smalley, "Evidence for charge transfer in doped carbon nanotube bundles form raman scattering," Nature, vol. 388, pp. 257-259, 1997.

[90] R. S. Lee, H. J. Kim, J. E. Fischer, A. Thess, and R. E. Smalley, "Conductivity enhancement in single-walled carbon nanotube bundles doped with $\mathrm{K}$ and $\mathrm{Br}$," Nature, vol. 388, pp. 255-257, 1997.

[91] S. Auvray, V. Derycke, M. Goffman, A. Filoramo, O. Jost, and J.-P. Bourgoin, "Chemical optimization of self-assembled carbon nanotube transistors," Nano Letters, vol. 5, pp. 451-455, 2005.

[92] J. Kong and H. Dai, "Full and modulated chemical gating of individual carbon nanotubes by organic amine compounds," Journal of Physical Chemistry B, vol. 105, pp. 2890-2893, 2001.

[93] K. Bradley, J.-C. P. Gabriel, and G. GrUner, "Flexible nanotube electronics," Nano Letters, vol. 3, pp. 1353-1355, 2003.

[94] I. Yamaguchi and T. Yamamoto, "Soluble self-doped single-walled carbon nanotube," Materials Letters, vol. 58, pp. 598-603, 2004.

[95] C. Klinke, J. Chen, A. Afzali, and P. Avouris, "Charge transfer induced polarity switching in carbon nanotube transistors," Nano Letters, vol. 5, pp. 555-558, 2005.

[96] A. Bachtold, P. Hadley, T. nakanishi, and C. Dekker, "Logic circuits with carbon nanotubes," in Structural and Electronic Properties of Molecular Nanostructures (H. Kuzmany, J. Fink, M. Mehring, and S. Roth, eds.), 633, (Kirchberg, Tirol, Austria), pp. 502-507, AIP conference Proceedings 633, 2002. 
[97] Y.-C. Tseng, P. Xuan, A. Javey, R. Malloy, Q. Wang, J. Bokor, and H. Dai, "Monolithic integration of carbon nanotube devices with silicon MOS technology," 2004.

[98] J. U. Lee, "Photovaltaic effect in ideal carbon nanotube diodes," Applied Physics Letters, vol. 87, pp. 073101 1-3, 2005.

[99] A. Nojeh, wai Kin Wong, A. W. Baum, and R. F. Pease, "Scanning electron microscopy of field-emitting individual single-walled carbon nanotubes," Applied physics Letters, vol. 85, pp. 112-114, 2004.

[100] Y. C. Kim, K. H. Sohn, Y. M. Cho, and E. H. Yoo, "Vertical alingment of printed carbon nanotubes by multiple field emission cycles," Applied Physics Letters, vol. 84, pp. 5350-5352, 2004.

[101] G. Pirio, P. Legagneux, D. Pribat, K. B. K. Teo, M. Chhowalla, G. A. J. Amaratunga, and W. I. Milne, "Fabrication and electrical characteristics of carbon nanotube field emission microcathodes with an integrated gate electrode," Nanotechnology, vol. 13, pp. 1-4, 2002.

[102] CNT@Cambridge, "http://www.-g.eng.cam.ac.uk/cnt/."

[103] C. N. Banwell and E. M. McCash, Fundamentals of Molecular Spectroscopy. Shoppenhangers Road, Maidenhead, Berkshire,SL6 2QL, England: McGraw Hill Publishing Company, fourth edition ed., 1994.

[104] R. Eisberg and R. Resnick, Quantum Physics of atoms molecules, solids, nuclei and particles: Second Edition. New York, USA: John Wiley and Sons, 1985.

[105] .M. S. Dresselhaus, G. Dresselhaus, A. Jorio, A. G. S. Filho, and R. Saito, "Raman spectroscopy on isolated single wall carbon nanotubes," Carbon, vol. 40, pp. 2043-2061, 2002.

[106] M. S. Dresselhaus, G. Dresselhaus, A. Jorio, A. G. S. Filho, G. G. Samsonidze, and R. Saito, "Science and applications of single-nanotube raman spectroscopy," Journal of Nanoscience and Nanotechnology, vol. 3, pp. 19-37, 2003.

[107] A. Jorio, G. Dresselhaus, M. S. Dresselhaus, M. Souza, M. S. S. Dantas, M. A. Pimenat, A. M. Rao, R. Saito, C. Liu, and H. M. Cheng, "Polarized raman study of single-wall semiconducting carbon nanotubes," Physical Review Letters, vol. 85, pp. 2617-2620, 2000.

[108] A. G. S. Filho, A. Jorio, G. G. Samsonidze, G. Dresselhaus, R. Saito, and M. S. Dresselhaus, "Raman spectroscopy for probing chemically/physically induced phenomena in carbon nanotubes," nanotechnology, vol. 14, pp. 1130-1139, 2003.

[109] A. Jorio, R. Saito, J. H. Hafner, C. M. Lieber, M. Hunter, T. McClure, G. Dresselhaus, and M. S. Dresselhaus, "Structural (n,m) determination of isolated singlewall carbon nanotubes by resonant raman scattering," Physical Review letters, vol. 86, pp. 1118-1121, 2001. 
[110] A. M. Rao, E. Richter, S. Bandow, B. Chase, P. C. Eklund, K. A. Williams, S. Fang, K. R. Subbaswamy, M. Menon, A. Thess, R. E. Smalley, G. Dresselhaus, and M. S. Dresselhaus, "Diameter-selective raman scattering from vibrational modes in carbon nanotubes," Science, vol. 275, pp. 187-191, 1997.

[111] M. S. Strano, "Probing chiral selective reactions using a revised kataura plot for the interpretation of single-walled carbon nanotubes," Journal of the American Chemical Society, vol. 125, pp. 16148-16153, 2003.

[112] M. S. Strano, C. A. Dyke, M. L. Usrey, P. W. Barone, M. J. Allen, H. Shan, C. Kittrell, R. H. Hague, J. M. Tour, and R. E. Smalley, "Elctronic structure control of single-walled carbon nanotube functionalization," Science, vol. 301, pp. 15191522, 2003.

[113] D. Briggs and M. P. Seah, Practical Surface Analysis by Auger and X-Ray photoelectron spectroscopy. John Wiley and Sons Ltd., 1987.

[114] J. F. Moulder, W. F. Stickle, P. E. Sobol, and K. D. Bomben, Handbook of X-Ray Photoelectron Spectroscopy. 6509 Flying Cloud Drive, Eden Prairie, Minnesota, 55344, USA: Perkin-Elmer Corporation, 1992.

[115] D. K. Schroder, Semiconductor Material and Device Characterization: second edition. New York, USA: John Wiley and Sons, 1998.

[116] "http://www.nist.gov/srd/nist20.html."

[117] L. Jiang, R. Cheung, R. Brown, and A. Mount, "Inductively coupled plasma etching of $\mathrm{SiC}$ in $\mathrm{SF}_{6} / \mathrm{O}_{2}$ and etch-induced surface chemical bonding modifications," Journal of Applied Physics, vol. 93, no. 3, pp. 1376-1383, 2003.

[118] K. H. An, J. G. Heo, K. G. Jeon, D. J. Bae, C. Jo, C. W. Yang, C.-Y. Park, and Y. H. Lee, "X-ray photoemission spectroscopy study of fluorinated single-walled carbon nanotubes," Applied Physics Letters, vol. 80, no. 22, pp. 4235-4237, 2002.

[119] G. Nanse, E. Papirer, P. Fioux, F. Moguet, and A. Tressaud, "Fluorination of carbon blacks: an X-ray photoelectron spectroscopy study: I.a literature review of XPS of fluorinated carbons. XPS investigayion of some reference compounds," Carbon, vol. 35, no. 2, pp. 175-194, 1997.

[120] R. Kwok, "Xps peak, software and user's guide,"

[121] S. Banerjee and S. S. Wong, "Rational sidewall functionalisation and purification of single-walled carbon nanotubes by solution-phased ozonolysis," Journal of physical chemistry B, vol. 106, pp. 12144-12151, 2002.

[122] Y. Joseph, N. Krasteva, I. Besnard, berit Guse, M. Rosenberger, U. Wild, A. Knop-Gericke, R. Schlogl, R. Krustev, A. Yasuda, and T. Vossmeyer, "Goldnanoparticle/organic linker films: self-assembly, electronic and structural characterisation, composition and vapour sensitivity," Faraday Discussions, vol. 125, pp. 77-97, 2003. 
[123] A. Gonzalez-Berrios, D. Huang, N. M. Medina-Emmanuelli, K. E. Kristian, O. O. Ortiz, J. A. Gonzalez, J. D. Jesus, I. M. vargas, B. R. Weiner, and G. Morell, "Effects of heavy-ion radiation on the electric fiels emission properties of sulfurdoped nanocomposite carbon films," Diamond and Related Materials, vol. 13, pp. 221-225, 2004.

[124] C. M. Whelan, M. R. Smyth, C. J. Barnes, N. M. D. Brown, and C. A. Anderson, "An XPS study of heterocyclic thiol self-assembly on Au(111)," Applied Surface Science, vol. 134, pp. 144-158, 1998.

[125] C. Vericat, M. E. Vela, G. Andreasen, and R. C. Salvarezza, "Sulfur-substrate interactions in spontaneously formed sulfur adlayers on $\mathrm{Au}(111)$, , Langmuir, vol. 17, pp. 4919-4924, 2001.

[126] J. C. Love, D. B. Wolfe, R. Haasch, M. L. Chabinyc, K. E. Paul, G. M. Whitesides, and R. G.Nuzzo, "Formation and structure of self-assembled monolayers of alkanethiolates on palladium," Journal of the American Chemical Society, vol. 125, pp. 2597-2609, 2003.

[127] S. M. Sze, Physics of Semiconductor Devices: Second Edition. John Wiley and Sons Inc., 1981.

[128] S. M. Sze, Semiconductor Devices Physics and Technology. John Wiley and Sons Inc., 1984.

[129] M. S. Tyagi, Introduction to semiconductor materials and devices. John Wiley and Sons, 1991.

[130] P. Avouris, "Molecular electronics with carbon nanotubes," Accounts of Chemical Research, vol. 35, pp. 1026-1034, 2002.

[131] E. H. Rhoderick, Metal-Semiconductor Contacts. Walton Street, Oxford, OX2 6DP: Oxford University Press, 1978.

[132] H. K. Henisch, Rectifying metal contacts. Amen House, London, E.C.4: Oxford University Press, 1957.

[133] H. K. Henisch, Semiconductor Contacts:An Approach to Ideas and Models. Walton Street, Oxford, OX2 6DP: Oxford University Press, 1984.

[134] D. M. Manos, Plasma Etching An Introduction. 24-28 Oval Road, London, NW1 7DX: Academic Press INC. (London)LTD., 1989.

[135] M. A. Lieberman and A. J. Lichtenberg, Principles of Plasma Discharges and materials processing. 1994.

[136] D. L. Flamm, V. M. Donnelly, and D. E. Ibbotson, "Basic chemistry and mechanisms of plasma etching," Journal of Vacuum Science and Technology B, vol. 1(1), p. 23, 1983.

[137] J. A. Mucha and D. W. Hess, "Plasma etching," in Introduction to Microlithography (L. F. Thomson, C. G. Wilson, and M. J. Bowden, eds.), pp. 215-285, Washington American Chemical Society, 1983. 
[138] D. L. Flamm and V. M. Donnelly, "The design of plasma etchants," Plamsa Chemistry and Plasma Processing, vol. 1(4), pp. 317-363, 1981.

[139] N. Hershkowitz, How a Langmuir Probe Works. Boston Academic Press, 1989.

[140] "www.scisys.com."

[141] V. M. Donnelly, D. L. Flamm, W. C. Dautermontsmith, and D. J. Werder, "Anisotropic etching of $\mathrm{SiO}_{2}$ in low frequency $\mathrm{CF}_{4} / \mathrm{O}_{2}$ and $\mathrm{NF}_{3} / \mathrm{Ar}$ plasmas," Journal of Applied Physics, vol. 55, 1984.

[142] P. R. Choudbury, ed., Handbook of Microlithography, Micromachining and Microfabrication. Volume 1. Michael Faraday House, Six Hills Way, Stevenage, Herts, SG1 2AY, UK: Institute of Electronic Engineers, 1997.

[143] H. Schmid and B. Michel, "Siloxane polymers for high-resolution, high-accuracy soft lithography," Macromolecules, vol. 33, pp. 3042-3049, 2000.

[144] B. D. Gates and G. M. Whitesides, "Replication of vertical features smaller than 2nm by soft lithography," Journal of the American Chemical Society, vol. 125, pp. 14986-14987, 2003.

[145] K. F. Kelly, I. W. Chiang, E. T. Mickelson, R. H. Hague, J. L. Margrave, X. Wang, G. E. Scuseria, C. Radloff, and N. J. Halas, "Insight into the mechanism of sidewall functionalisation of single-walled nanotubes: an STM study," Chemical Physics Letters, vol. 313, pp. 445-450, 1999.

[146] K. Ishikawa, S. K. M. Okigawa, M. Sekine, S. Yamasaki, T. Yasuda, and J. ichi Isoya, "In vacuo electron-spin-resonance study on amorphous fluorinated carbon films for understanding of surface chemical reactions in plasma etching," Applied Physics Letters, vol. 81, no. 10, pp. 1773-1775, 2002.

[147] B. N. Khare, M. Meyyappan, A. M. Cassell, C. V. Nguyen, and J. Han, "Functionalisation of carbon nanotubes using atomic hydrogen from a glow discharge," Nano Letters, vol. 2, no. 1, pp. 73-77, 2002.

[148] B. N. Khare, M. Meyyappan, J. Kralj, P. White, M. Sisay, H. Imanaka, J. Koehne, and J. Charles W. Baushchlicher, "A glow discharge approach for functionalization of carbon nanotubes," Applied Physics Letters, vol. 81, no. 27, pp. 52375239, 2002.

[149] H. D. Klotz, R. Mach, F. Oleszak, H. E. Maneck, H. Georing, and K. W. Brzezinka, "Synthesis and characterisation of nanoscaled and nanostructured carbon containing materials produced by thermal plasma technology," Applied Surface Science, vol. 179, pp. 1-7, 2001.

[150] A. Kukovecz, C. Kramberger, M. Holzinger, H. Kuzmany, J. Schalko, M. Mannsberger, and A. Hirsch, "On the stacking behaviour of functionalised single-walled carbon nanotubes," Journal of physical Chemistry B, vol. 106, pp. 6374-6380, 2002. 
[151] H. Kataura, Y. Kumazawa, Y. Maniwa, I. Umezu, S. Suzuki, Y. Ohtsuka, and Y. Achiba, "Optical properties of single-wall carbon nanotubes," Synthetic Materials, vol. 103, pp. 2555-2558, 1999.

[152] H. Kuzmany, B. Burger, A. Thess, and R. E. Smalley, "Vibrational spectra of single wall carbon nanotubes," Carbon, vol. 36, no. 5-6, pp. 709-712, 1998.

[153] A. V. Krasheninnikov, K. Nordlund, and J. Keinonen, "Ion-irradiation-induced welding of carbon nanotubes," Physical Review B, vol. 66, pp. 245403 1-6, 2002.

[154] Y. Hattori, Y. Watanbe, S. Kawasaki, F. Okino, B. K. Pradham, T. Kyotani, A. Tomita, and $\mathrm{H}$. Touhara, "Carbon-alloying of the rear surfaces of nanotubes by direct fluorination," Carbon, vol. 37, pp. 1033-1038, 1999.

[155] W. Schwarzenbach, A. Tserepi, J. Dreouard, and N. Sadeghi, "Mass spectromter detection of $\mathrm{F}$ atoms and $\mathrm{CF}_{x}$ radicals in $\mathrm{CF}_{4}$ plasmas," Japan Journal of Applied Physics, vol. 36, pp. $4644-4647,1997$.

[156] W. Schwarzenbach, G. Cunge, and J. P. Booth, "High mass positive ions and molecules in capacitively-coupled radio-frequncy $\mathrm{CF}_{4}$ plasmas," Journal of Applied Physics, vol. 85, pp. 7562-7568, 1999.

[157] P. Fendel, A. Francis, and U. Czarnetzki, "Sources and sinks of $\mathrm{CF}$ and $\mathrm{CF}_{2}$ in a cc-RF $\mathrm{CF}_{4}$-plasma under various condictions," Plamsa Sources Science and Technology, vol. 14, pp. 1-11, 2005.

[158] Z. Gu, H. Peng, R. H. Hauge, R. E. Smalley, and J. L. Margrave, "Cutting single-wall carbon nanotubes through fluorination," Nano Letters, vol. 2, no. 9, pp. 1009-1013, 2002.

[159] K. Xiao, Y. Liu, P. Hu, G. Yu, Y. Sun, and D. Zhu, "n-type field effect transistors made of an individual nitrogen-doped multiwalled carbon nanotube," Journal of the American Chemical Society, vol. 127, pp. 8614-8617, 2005.

[160] P. Hu, K. Xiao, Y. Liu, G. Yu, X. Wang, L. Fu, G. Cui, and D. Zhu, "Multiwall nanotubes with intramolecular junctions $\left(\mathrm{cn}_{x} / \mathrm{c}\right)$ : Preparation rectification, logic gates and application," Applied Physics Letters, vol. 84, pp. 4932-4934.

[161] B. Wei, R. Spolenak, P. Kohler-Redlich, M. Ruhle, and E. Arzt, "Electrical transport in pure and boron-doped carbon nanotubes," Applied Physics Letters, vol. 74, no. 21, pp. 3149-3151, 1999.

[162] S. M. Kane and J. L. Gland, "Cyclohexanethiol adsorption and reaction on the ni(100) surface," Surface Science, vol. 468, pp. 101-108, 2000.

[163] M. C. Coen, B. Keller, P. Groening, and L. Sclapbach, "Functionalization of graphite, glassy carbon, and polymer surfaces with highly oxidized sulfur species by plasma treatments," Journal of Applied Physics, vol. 92, pp. 5077-5083, 2002.

[164] J. Heeg, C. Kramer, M. Wolter, S. Michaelis, W. Plieth, and W. J. Fischer, "Polythiophene $-\mathrm{O}_{3}$ surface reactions studied by XPS," Applied Surface Science, vol. 180, pp. 36-41, 2001. 
[165] S. Glenis, A. J. Nelson, and M. M. Labes, "Sulfur doped graphite prepared via arc discharge of carbon rods in the presence of thiophenes," Journal of Applied physics, vol. 86, pp. 4464-4466, 1999.

[166] R. Kalish, C. Uzan-Saguy, R. Walker, and S. Prawer, "Electrically active sulfurdefect complexes in sulfur implanted diamond," Journal of Applied Physics, vol. 94, pp. 3923-3930, 2003.

[167] S. Gupta, B. R. Weiner, and G. Morell, "Ex situ spectroscopic ellipsometry and raman spectroscopy investigations of chemical vapor deposited sulfur incorporated nanocrystalline carbon thin films," Journal of Applied Physics, vol. 92, pp. 54575462, 2002.

[168] S. Gupta, B. R. Weiner, and G. Morell, "Investigations of the electron filed emission properties and microstructure correlation in sulfur-incorporated nanocrystalline carbon thin filma," Journal of Applied Physics, vol. 91, pp. 10088-10097, 2002.

[169] M. Zhang, M. Yudasaka, and S. Iijima, "Production of large-diameter single-wall carbon nanotubes by adding Fe to a NiCo catalyst in laser ablation," Journal of Physical Chemistry B, vol. 108, pp. 12757-12762, 2004.

[170] M. D. Lay, J. P. Novak, and E. S. Snow, "Simple route to large-scale ordered arrays of liquid-deposited carbon nanotubes," Nano Letters, vol. 4, pp. 603-606, 2004.

[171] J. C. Lewenstein, T. P. Burgin, A. Ribayrol, L. A. Nagahara, and R. K. Tsui, "Highyield selective placement of carbon nanotubes on pre-patterned electrodes," Nano Letters, vol. 2, pp. 443-446, 2002.

[172] J. Zhu, M. Yudasaka, M. Zhang, D. Kasuya, and S. Iijima, "A surface modification approach to the patterned self-assembly of single-walled carbon nanomaterials," Nano Letters, vol. 3, pp. 1239-1243, 2003.

[173] M. J. Biercuk, N. Mason, and C. M. Marcus, "Local gating of carbon nanotubes," Nano Letters, vol. 4, pp. 1-4, 2004.

[174] R. Martel, T. Schmidt, H. R. Shea, T. Hertel, and P. Avouris, "Single- and multiwall carbon nanotube field effect transistors," Applied Physics Letters, vol. 75, no. 17, pp. 2447-2449, 1998.

[175] N. O. V. Plank, R. Cheung, and R. J. Andrews, "Thiolation of single-wall carbon nanotubes and their self-assembly," Applied Physics Letters, vol. 85, pp. 32293231, 2004. 